\title{
Sulfonate Versus Sulfonate: Nickel and Palladium Multimetallic Cross-Electrophile Coupling of Aryl Triflates with Aryl Tosylates
}

\author{
Kai Kang, Liangbin Huang, and Daniel J. Weix*
}

\section{Supporting Information}

1. General Information

2. Reaction Optimization

3. Synthesis of Starting Materials

3.1 Synthesis of Aryl Triflates

3.2 Synthesis of Aryl Tosylates

3.3 Synthesis of Other Phenol Derivatives

3.4 Availability Analysis of Selected Phenols and Their Aryl Halides (Halide = Cl, Br, I) Counterparts

4. Cross-Electrophile Couplings of Phenol Derivatives under Nickel and Palladium Multimetallic Conditions

4.1 Cross-Electrophile Couplings of Aryl Triflates with Aryl Tosylates

4.2 Cross-Electrophile Couplings between Selected Phenol Derivatives

4.3 Cross-Electrophile Couplings of $p$-Anisyl Triflate (1a) with Selected Aryl Arenesulfonate Esters

4.4 Hammett Constants of Substituents on Selected Aryl Triflates and Aryl Tosylates and Their Influence on the Yield of Cross-Coupled Product

4.5 Aryl Sulfonate Esters That Are Not Suitable for Nickel and Palladium Multimetallic Conditions

\section{Mechanistic Studies}

5.1 Background Reactions of Zn with $p$-Anisyl Triflate (1a) and Phenyl Tosylate (2a)

5.2 Background Reactions of LiBr with $p$-Anisyl Triflate (1a) and Phenyl Tosylate (2a)

5.3 Iodine Quenching Experiments for the Reactions of $p$-Anisyl Triflate (1a) with Phenyl Tosylate (2a) in the Presence of Nickel and/or Palladium Catalysts

5.4 Competitive Oxidative Additions of $p$-Anisyl Triflate (1a) and Phenyl Tosylate (2a) to (L1) $\mathrm{Ni}^{0}(\operatorname{cod})$ 


\section{General Information}

\section{Reagents}

Catalysts and Reductants: All metal catalysts and metal reductants, unless otherwise noted, were stored and handled in a $\mathrm{N}_{2}$ filled glovebox. Palladium(II) dichloride $\left(\mathrm{PdCl}_{2}\right)$ was purchased from Alfa Aesar. Nickel(II) dichloride ethylene glycol dimethyl ether complex $\left(\mathrm{NiCl}_{2}(\mathrm{dme})\right)$ was purchased from SigmaAldrich. The reductant zinc powder (-325 mesh, 97\%), zinc flake (-325 mesh, 99.9\% metal basis), and manganese powder (-325 mesh) were purchased from Alfa Aesar. Zinc powder was used as the reductant for all the reactions unless otherwise noted.

Ligands: The optimal ligand for the nickel catalyst, 4,4'-diphenyl-2,2'-bipyridine, was purchased from Santa Cruz Biotechnology, Inc. Other amine ligands used for reaction optimization, 2,2':6',2"-terpyridine, 1,10-phenanthroline (phen), 2,2'-bipyridine (bpy), 4,4'-di-tert-butyl-2,2'-bipyridine, dimethyl 2,2'bipyridine-4,4'-dicarboxylate, 6,6'-dimethyl-2,2'-bipyridine and 4,4'-dimethoxy-2,2'-bipyridine were purchased from Sigma-Aldrich or Alfa Aesar. The optimal ligand for the palladium catalyst, 1,4bis(diphenylphosphino)butane (dppb), was purchased from Alfa Aesar. Other phosphine ligands used for reaction optimization, 1,3-bis(diphenylphosphino)propane (dppp), 1,2-bis(diphenylphosphino)ethane (dppe), triphenylphosphine $\left(\mathrm{PPh}_{3}\right)$, tricyclohexylphosphine ( $\left.\mathrm{PCy}_{3}\right)$, 4,5-bis(diphenylphosphino)-9,9dimethylxanthene (Xantphos), 1,1'-bis(diphenylphosphino)ferrocene (dppf), 2-dicyclohexylphosphino2',4',6'-triisopropylbiphenyl (XPhos) were purchased from Sigma-Aldrich or Alfa Aesar.

Salt Additives: Lithium bromide $(\mathrm{LiBr})$, sodium bromide $(\mathrm{NaBr})$, potassium bromide $(\mathrm{KBr})$, tetra- $n$ butylammonium bromide (TBAB), lithium chloride ( $\mathrm{LiCl})$, and zinc bromide $\left(\mathrm{ZnBr}_{2}\right)$ were purchased from Alfa Aesar. All additives were used as received without further purification.

Solvents for Reactions: Anhydrous $N, N$-dimethylformamide (DMF, 99.9\%) was purchased from SigmaAldrich. It was opened and stored in a $\mathrm{N}_{2}$ filled glovebox. Dry dichloromethane (DCM) used for synthesis of starting materials was purchased from Fisher Chemical and purified by inert solvent purification system (PureSol MD 5) before use.

Starting Materials: Most aryl electrophiles used are known compounds and were synthesized according to reported procedures. Preparations and characterizations of unreported starting materials $1 \mathbf{i}, \mathbf{2 o}, 7 \mathbf{c}, \mathbf{7 f}$ and $7 \mathrm{~g}$ are described below in the synthesis of starting materials.

\section{Methods}

NMR Spectroscopy: ${ }^{1} \mathrm{H}$ nuclear magnetic resonance (NMR) spectroscopy chemical shifts are reported in ppm and referenced to tetramethylsilane (TMS) in solvent (for $\mathrm{CDCl}_{3} \delta=0 \mathrm{ppm}$ ) or the residual solvent peaks $\left(\delta=7.26 \mathrm{ppm}\right.$ for $\mathrm{CDCl}_{3}, \delta=7.15$ ppm for $\mathrm{C}_{6} \mathrm{D}_{6}$, and $\delta=2.50 \mathrm{ppm}$ for DMSO-d $\mathrm{d}_{6}$ ). For ${ }^{13} \mathrm{C}$ NMR chemical shifts, the residual solvent peak $\left(\delta=77.20 \mathrm{ppm}\right.$ for $\mathrm{CDCl}_{3}, \delta=128.06 \mathrm{ppm}$ for $\mathrm{C}_{6} \mathrm{D}_{6}$, and $\delta=$ 
$39.52 \mathrm{ppm}$ for DMSO- $\mathrm{d}_{6}$ ) were used as references. For ${ }^{19} \mathrm{~F}$ NMR chemical shifts, the external standard, $\alpha, \alpha, \alpha$-trifluorotoluene $(\delta=0 \mathrm{ppm})$, was used as the reference. NMR spectra were recorded on Avance Bruker NMR spectrometers operating at either $400 \mathrm{MHz}$ or $500 \mathrm{MHz}$ and data analysis was performed with MestreNova. Chemical shifts are reported in parts per million (ppm), multiplicities are indicated by singlet $(\mathrm{s})$, doublet (d), triplet (t), quartet (q), multiplet (m) and broad (br). Coupling constants $(J)$ are reported in Hertz (Hz).

Gas Chromatography: GC analyses were performed on an Agilent 7890A GC equipped with dual DB-5 columns $(20 \mathrm{~m} \times 180 \mu \mathrm{m} \times 0.18 \mu \mathrm{m})$, dual FID detectors, and hydrogen as the carrier gas. A sample volume of $1 \mu \mathrm{L}$ was injected at a temperature of $300{ }^{\circ} \mathrm{C}$ and a $100: 1$ split ratio. The initial inlet pressure was 20.3 psi but varied as the column flow was held constant at $1.8 \mathrm{~mL} / \mathrm{min}$ for the duration of the run. The initial oven temperature of $50{ }^{\circ} \mathrm{C}$ was held for $0.46 \mathrm{~min}$ followed by a temperature ramp of $65^{\circ} \mathrm{C} / \mathrm{min}$ up to $300{ }^{\circ} \mathrm{C}$. The temperature was held at $300{ }^{\circ} \mathrm{C}$ for $3 \mathrm{~min}$. The total run time was $\sim 7.3 \mathrm{~min}$ and the FID temperature was $325^{\circ} \mathrm{C}$.

Mass Spectrometry: Mass spectrometry data was collected on a Thermo Q Exactive Plus (thermofisher.com) via flow injection with electrospray ionization or via ASAP-MS (asap-ms.com) by the chemistry mass spectrometry facility at the University of Wisconsin-Madison. The purchase of the Thermo Q Exactive Plus in 2015 was funded by NIH Award 1S10 OD020022 to the Department of Chemistry.

Supercritical Fluid Chromatography Mass Spectrometry: SFC/MS analyses were performed on a Waters ACQUITY UPC2 equipped with ACQUITY UPC2 PDA and ACQUITY QDa Detector. A Daicel Dcpack SFC-A column ( $3 \mathrm{~mm}$ ID $\times 150 \mathrm{~mm} \mathrm{~L}, 3 \mu \mathrm{m}$ PS) was used for separations. The eluent was a mixture $\left(97: 3 \mathrm{CO}_{2} / \mathrm{MeOH}\right)$ with a flow rate of $2 \mathrm{~mL} / \mathrm{min}$ at $40{ }^{\circ} \mathrm{C}$ with an ABPR at $1500 \mathrm{psi}$.

Infrared Spectroscopy: Solid state FT-IR spectroscopic data were collected in ATR mode using a Bruker TENSOR 27 spectrometer located in the Chemical Instrumentation Instructional Laboratory at the University of Wisconsin-Madison Department of Chemistry and are reported in wavenumbers $\left(\mathrm{cm}^{-1}\right)$.

Flash Chromatography for Isolations: Chromatography was performed on silica gel (EMD, silica gel 60, particle size 0.040-0.063 mm) using standard flash techniques or on 40-100 g HP Silica column using a Teledyne Isco Rf-200 (detection at 210 and $254 \mathrm{~nm}$ ). Products were visualized by UV.

Melting Point: Melting points were measured using a DigiMelt M160 Melting Point Apparatus. 


\section{Reaction Optimization}

\subsection{General Procedure for Optimization Studies (Table S1, entries 1-20, 22-35 and 37)}

The following procedures were conducted in a $\mathrm{N}_{2}$ filled glovebox for convenience.

Preparation of nickel and palladium catalyst stock solutions (0.025 $\mathrm{M}$ with respect to $\mathrm{Ni}$ and Pd)

An oven-dried 1-dram vial equipped with a PTFE-coated stir-bar was charged with $\mathrm{NiCl}_{2}(\mathrm{dme})$ (11.0 mg, $0.050 \mathrm{mmol}$ ) and the listed amine ligand (L1-L7, $0.060 \mathrm{mmol})$. DMF (2.0 mL) was then added before the dram vial was capped with a screw cap fitted with a PTFE-faced silicone septum. The mixture was stirred (1000 rpm) at $\mathrm{rt}$ for $12 \mathrm{~h}$ before being used (vide infra). A second oven-dried 1-dram vial equipped with a PTFE-coated stir-bar was charged with $\mathrm{PdCl}_{2}(8.9 \mathrm{mg}, 0.050 \mathrm{mmol})$ and the listed phosphine ligand (0.060 mmol for dppb or L8-L11 and 0.120 mmol for L12-L14). DMF (2.0 mL) was then added before the dram vial was capped with a screw cap fitted with a PTFE-faced silicone septum. The mixture was stirred (1000 rpm) at $\mathrm{rt}$ for $12 \mathrm{~h}$ before being used (vide infra).

\section{Catalytic reaction setup}

An oven-dried 1-dram vial equipped with a PTFE-coated stir-bar was charged sequentially with $1 \mathbf{a}$ (64 mg, $0.25 \mathrm{mmol}, 1.0$ equiv), $\mathbf{1 b}(62 \mathrm{mg}, 0.25 \mathrm{mmol}, 1.0$ equiv), the listed salt additive ( 0 to $1.0 \mathrm{mmol}$, 0 to 4.0 equiv), and the listed reductant ( $\mathrm{Zn}$ or $\mathrm{Mn}, 0.50 \mathrm{mmol}, 2.0$ equiv). Aliquots from both the $0.025 \mathrm{M}$ nickel and palladium stock solutions (vide supra) were then added (30, 100 or $500 \mu \mathrm{L}$ for $0.3,1.0$ or 5.0 mol\% catalyst loading, respectively), followed by dodecane as an internal standard (15-20 mg). Sufficient DMF was then added to bring the total volume of solvent to $1.0 \mathrm{~mL}$. The vial was capped with a screw cap fitted with a PTFE-faced silicone septum, and the mixture was stirred (1000 rpm) for $5 \mathrm{~min}$ in the glovebox at $\mathrm{rt}$. The reaction vial was then removed from the glovebox and heated in a reaction block set to the desired temperature (rt, 40 , or $60^{\circ} \mathrm{C}$ ) with stirring $(1250 \mathrm{rpm})$ for $14 \mathrm{~h}$.

\section{GC analysis}

After $14 \mathrm{~h}$, the reaction mixture $(1 \mathrm{~mL})$ was cooled to $\mathrm{rt}$, diluted with ethyl acetate $(5 \mathrm{~mL})$, and filtered through a 2-cm silica gel plug in a Pasteur pipette. A $100 \mu \mathrm{L}$ aliquot of the resulting solution was added to a GC vial and further diluted with ethyl acetate $(1 \mathrm{~mL})$. The sample was analyzed by GC using our standard method and the yields were determined based on the peak area of the analyte compared to dodecane as an internal standard. 


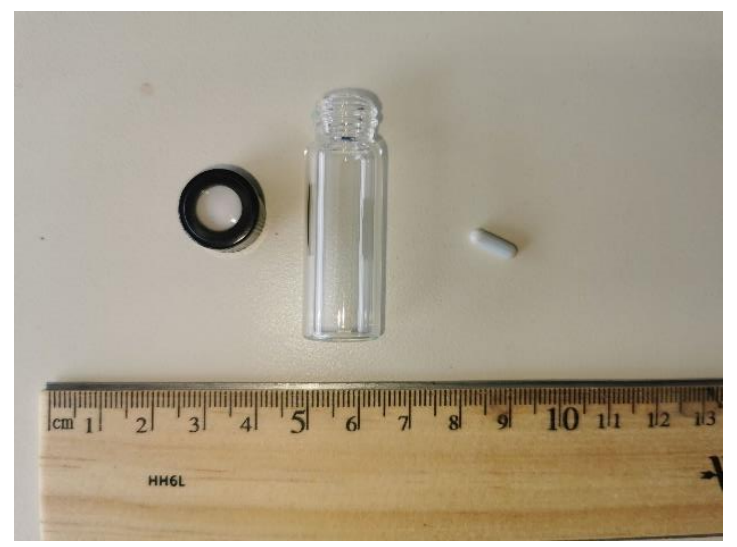

Figure S1. Screw cap fitted with a PTFE-faced silicone septum, 1-dram vial, and PTFE-coated stir-bar used in optimization studies and for isolated yields at up to $0.5 \mathrm{mmol}$ scale.

\subsection{Procedure for Benchtop Reaction Setup (Table S1, entry 21)}

The following procedures were performed on the bench using standard Schlenk techniques and $\mathrm{N}_{2}$ as the inert gas.

\section{Preparation of nickel and palladium catalyst stock solutions $(0.025 \mathrm{M}$ with respect to $\mathrm{Ni}$ and $\mathrm{Pd})$}

A 1-dram vial equipped with a PTFE-coated stir-bar was charged with $\mathrm{NiCl}_{2}$ (dme) (11.0 mg, 0.050 mmol) and $\mathbf{L 1}$ (18.5 mg, $0.060 \mathrm{mmol}$ ) before it was capped with a screw cap fitted with a PTFE-faced silicone septum. The vial was connected to a Schlenk line via a needle through the septum. A second venting needle (open to air) was added, and the vial was purged with $\mathrm{N}_{2}$ for $5 \mathrm{~min}$. DMF (2.0 mL) was then added via syringe through the septum cap. The venting needle was removed, and the mixture was stirred (1000 $\mathrm{rpm}$ ) under positive $\mathrm{N}_{2}$ pressure at $\mathrm{rt}$ for $12 \mathrm{~h}$ before being used (vide infra). A second 1-dram vial with a PTFE-coated stir-bar was charged with $\mathrm{PdCl}_{2}(8.9 \mathrm{mg}, 0.050 \mathrm{mmol})$ and dppb (25.6 $\left.\mathrm{mg}, 0.060 \mathrm{mmol}\right)$ before it was capped with a screw cap fitted with a PTFE-faced silicone septum. The vial was connected to a Schlenk line via a needle through the septum. A second venting needle (open to air) was added, and the vial was purged with $\mathrm{N}_{2}$ for $5 \mathrm{~min}$. DMF $(2.0 \mathrm{~mL}$ ) was then added via syringe through the septum cap. The venting needle was removed, and the mixture was stirred $(1000 \mathrm{rpm})$ under positive $\mathrm{N}_{2}$ pressure at $\mathrm{rt}$ for 12 $\mathrm{h}$ before being used (vide infra).

\section{Catalytic reaction setup}

A $10 \mathrm{~mL}$ Schlenk tube (Figure S2, left) equipped with a PTFE-coated stir-bar was charged sequentially with $\mathbf{1 a}(128 \mathrm{mg}, 0.50 \mathrm{mmol}, 1.0$ equiv), $1 \mathbf{b}(124 \mathrm{mg}, 0.50 \mathrm{mmol}, 1.0$ equiv), $\mathrm{LiBr}(174 \mathrm{mg}$, $2.0 \mathrm{mmol}, 4.0$ equiv) and $\mathrm{Zn}(65 \mathrm{mg}, 1.0 \mathrm{mmol}, 2.0$ equiv). The reaction tube was fitted with a rubber septum on the joint and the hose adapter was connected to a $\mathrm{N}_{2}$ Schlenk line. The vessel was subjected to three evacuation/ $\mathrm{N}_{2}$ fill cycles. Under positive $\mathrm{N}_{2}$ pressure, nickel catalyst stock solution (200 $\mu \mathrm{L}$ for 1.0 mol\% catalyst loading), palladium catalyst stock solution (200 $\mu \mathrm{L}$ for $1.0 \mathrm{~mol} \%$ catalyst loading), and DMF 
$(1.6 \mathrm{~mL})$ were added by syringe through the septum cap. The vessel was sealed by closing the stopcock on the Schlenk flask, and the reaction mixture was stirred $(1000 \mathrm{rpm})$ at $\mathrm{rt}$ for $10 \mathrm{~min}$. The vessel was then placed into a $40{ }^{\circ} \mathrm{C}$ oil bath, and the mixture was stirred (1000 rpm) for $24 \mathrm{~h}$ (Figure S2, right).

\section{Isolation and purification}

When the reaction was judged complete (GC analysis), the reaction mixture was diluted with ethyl acetate $(10 \mathrm{~mL})$, filtered through a short plug of silica gel $(1.5 \mathrm{~cm} \times 3.0 \mathrm{~cm})$, and the vessel and silica were washed with extra portions of ethyl acetate $(15 \mathrm{~mL} \times 5)$. Silica gel (2.5-3.0 g) was then added to the combined filtrate and the volatile solvents were removed by rotary evaporation. The resulting dry-loaded material was purified by flash chromatography on silica gel to provide the desired product.

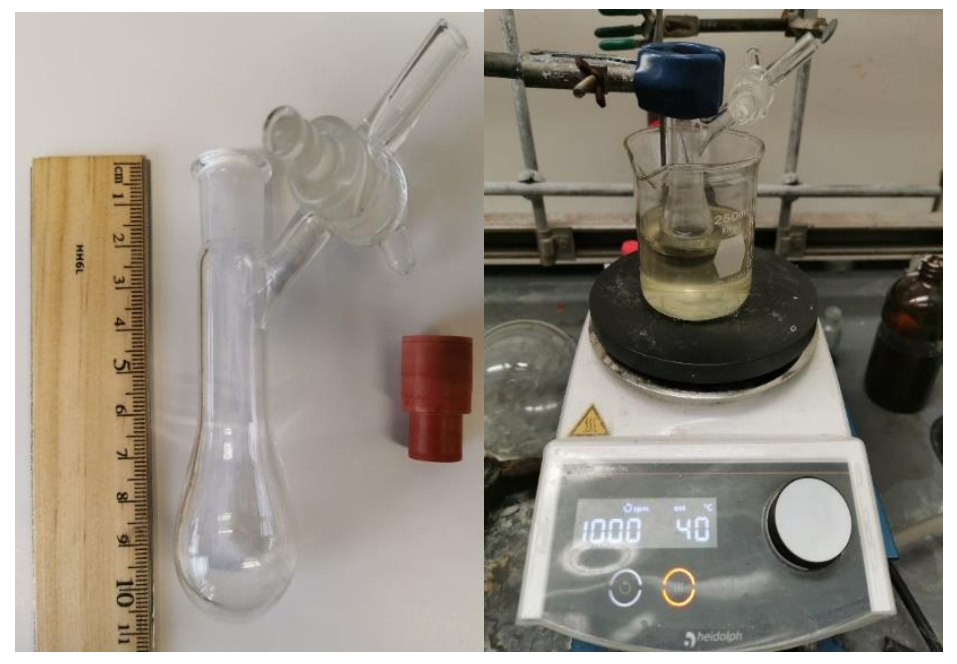

Figure S2: (Left) the Schlenk tube and rubber septum used in the benchtop reaction (Table S1, entry 21); (Right) the reaction vessel in the oil bath (Table S1, entry 21).

\subsection{Procedure for Lower Catalyst Loading Reactions (0.5 mol\% Ni/Pd, $0.6 \mathrm{~mol} \%$ ligands) (Table S1, entry 36)}

The following procedures were conducted in a $\mathrm{N}_{2}$ filled glovebox for convenience. Nickel and palladium catalyst stock solutions were prepared following general procedure for optimization studies (page S4) using $\mathbf{L 1}$ (18.5 mg, $0.060 \mathrm{mmol}$ ) as the amine ligand and dppb (25.6 $\mathrm{mg}, 0.060 \mathrm{mmol}$ ) as the phosphine ligand. GC analysis was performed following general procedure for optimization studies (page S4) after $24 \mathrm{~h}$ reaction time.

\section{Catalytic Reaction Setup}

An oven-dried 1-dram vial equipped with a PTFE-coated stir-bar was charged sequentially with 1a (128 mg, 0.50 mmol, 1.0 equiv), 1 b (124 mg, 0.50 mmol, 1.0 equiv), LiBr (174 mg, 2.0 mmol, 4.0 equiv), and $\mathrm{Zn}$ (65 mg, $1.0 \mathrm{mmol}, 2.0$ equiv). Aliquots from both the $0.025 \mathrm{M}$ nickel (100 $\mu \mathrm{L}$ for $0.5 \mathrm{~mol} \%$ ) and 
palladium (100 $\mu \mathrm{L}$ for $0.5 \mathrm{~mol} \%$ ) stock solutions were added, followed by dodecane as an internal standard (15-20 mg). DMF (0.8 mL) was added before the vial was capped with a screw cap fitted with a PTFEfaced silicone septum. The reaction mixture was stirred $(1000 \mathrm{rpm})$ for $10 \mathrm{~min}$ in the glovebox at $\mathrm{rt}$. The vial was then removed from the glovebox and heated in a reaction block set to $40{ }^{\circ} \mathrm{C}$ with stirring (1250 rpm) for $24 \mathrm{~h}$. 
Table S1. Optimization of reaction parameters for cross-electrophile coupling of $\mathbf{1 a}$ with $\mathbf{2} \mathbf{a}^{a, b}$

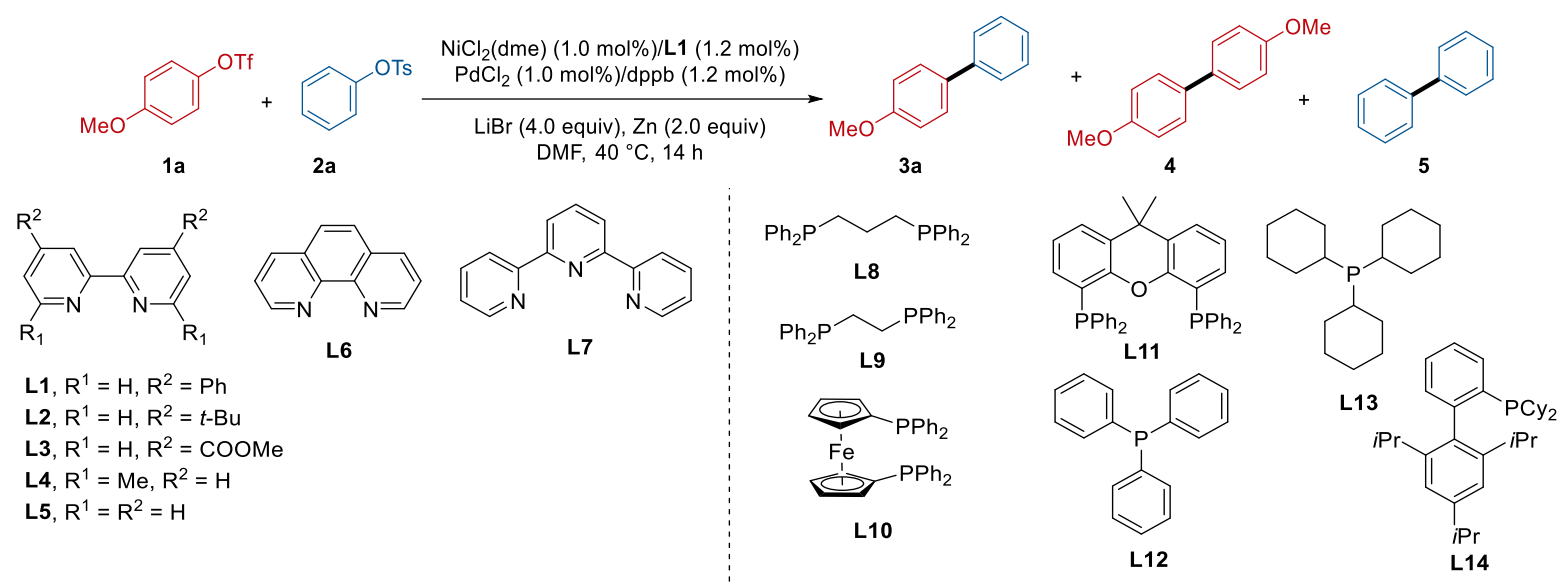

\begin{tabular}{|c|c|c|c|c|c|c|}
\hline entry & variations from conditions above & $\mathbf{3 a}(\%)$ & $4(\%)$ & $5(\%)$ & $\mathbf{1 a}(\%)$ & $\mathbf{2 a}(\%)$ \\
\hline 1 & none & 76 & 12 & 13 & 0 & 0 \\
\hline 2 & without $\mathrm{PdCl}_{2}$ and dppb & 58 & 20 & 18 & 0 & 0 \\
\hline 3 & without $\mathrm{NiCl}_{2}(\mathrm{dme})$ and $\mathbf{L} \mathbf{1}$ & $<5$ & 0 & 0 & 93 & 95 \\
\hline 4 & without $\mathrm{PdCl}_{2}$ & 19 & 11 & 6 & 49 & 48 \\
\hline 5 & without $\mathrm{NiCl}_{2}(\mathrm{dme})$ & 0 & 0 & 0 & 98 & 96 \\
\hline 6 & without L1 & 0 & 0 & 0 & 98 & 98 \\
\hline 7 & without dppb & 49 & 13 & $<5$ & 13 & 30 \\
\hline 8 & without $\mathrm{Zn}$ & 0 & 0 & 0 & 95 & 95 \\
\hline 9 & Mn instead of $\mathrm{Zn}$ & $<5$ & 0 & 0 & 91 & 95 \\
\hline 10 & without $\mathrm{LiBr}$ & 8 & 3 & 1 & 82 & 91 \\
\hline 11 & 2.0 equiv of $\mathrm{LiBr}$ & 63 & 12 & 5 & 11 & 25 \\
\hline 12 & $\mathrm{NaBr}$ instead of $\mathrm{LiBr}$ & 72 & 12 & 9 & 0 & 0 \\
\hline 13 & $\mathrm{KBr}$ instead of $\mathrm{LiBr}$ & 73 & 12 & 5 & 0 & 9 \\
\hline 14 & $n-\mathrm{Bu}_{4} \mathrm{NBr}$ instead of $\mathrm{LiBr}$ & 54 & 11 & 8 & 0 & 9 \\
\hline 15 & $\mathrm{LiF}$ instead of $\mathrm{LiBr}$ & 5 & $<5$ & 0 & 83 & 92 \\
\hline 16 & $\mathrm{LiBF}_{4}$ instead of $\mathrm{LiBr}$ & 9 & $<5$ & 0 & 81 & 91 \\
\hline 17 & $\mathrm{LiCl}$ instead of $\mathrm{LiBr}$ & 60 & 18 & 18 & 0 & 0 \\
\hline 18 & $\mathrm{ZnBr}_{2}$ instead of $\mathrm{LiBr}$ & 0 & 0 & 0 & 96 & 98 \\
\hline 19 & $\mathrm{rt}$ & 64 & 10 & 6 & 9 & 6 \\
\hline 20 & $60^{\circ} \mathrm{C}$ & 67 & 16 & 10 & 0 & 0 \\
\hline 21 & Reaction setup on benchtop & $73^{c}$ & l & l & l & I \\
\hline 22 & $\mathbf{L 2}$ instead of $\mathbf{L 1}$ & 32 & 6 & 8 & 43 & 48 \\
\hline 23 & L3 instead of L1 & 12 & 48 & $<5$ & 0 & 58 \\
\hline 24 & L4 instead of L1 & 8 & 8 & $<5$ & 79 & 83 \\
\hline 25 & L5 instead of L1 & 65 & 18 & 15 & 0 & 0 \\
\hline 26 & L6 instead of L1 & 62 & 19 & 14 & 0 & 5 \\
\hline 27 & L7 instead of L1 & $<5$ & 8 & $<5$ & 84 & 76 \\
\hline 28 & L8 instead of dppb & 41 & 24 & 20 & 0 & 0 \\
\hline 29 & L9 instead of dppb & 61 & 19 & 13 & 0 & 0 \\
\hline 30 & L10 instead of dppb & 59 & 20 & 10 & 0 & 0 \\
\hline 31 & L11 instead of dppb & 43 & 27 & 13 & 0 & 0 \\
\hline 32 & L12 instead of dppb & 43 & 29 & 11 & 0 & 0 \\
\hline 33 & L13 instead of dppb & 29 & 33 & 27 & 0 & 0 \\
\hline 34 & L14 instead of dppb & 55 & 24 & 16 & 0 & 0 \\
\hline 35 & $\begin{array}{l}5.0 \mathrm{~mol} \% \mathrm{NiCl}_{2}(\mathrm{dme}) / 6.0 \mathrm{~mol} \% \mathbf{L 1} \text { and } \\
5.0 \mathrm{~mol} \% \mathrm{PdCl}_{2} / 6.0 \mathrm{~mol} \% \mathrm{dppb}\end{array}$ & 64 & 16 & 21 & 0 & 0 \\
\hline 36 & $\begin{array}{l}0.5 \mathrm{~mol}^{2} \mathrm{NiCl}_{2}(\mathrm{dme}) / 0.6 \mathrm{~mol} \% \mathrm{L1} \text { and } \\
0.5 \mathrm{~mol} \% \mathrm{PdCl}_{2} / 0.6 \mathrm{~mol} \% \mathrm{dppb}\end{array}$ & $73^{d}$ & 12 & 12 & 0 & 0 \\
\hline 37 & $\begin{array}{l}0.30 \mathrm{~mol} \% \mathrm{NiCl}_{2}(\mathrm{dme}) / 0.36 \mathrm{~mol} \% \mathrm{L1} \text { and } \\
0.30 \mathrm{~mol} \% \mathrm{PdCl}_{2} / 0.36 \mathrm{~mol} \% \mathrm{dppb}\end{array}$ & 58 & 8 & 5 & 20 & 17 \\
\hline
\end{tabular}

${ }^{a}$ Reactions were run on a $0.25 \mathrm{mmol}$ scale in $1.0 \mathrm{~mL}$ DMF. DMF $=N, N$-dimethylformamide. ${ }^{b} \mathrm{GC}$ yields using dodecane as an internal standard. ${ }^{c}$ Isolated yield from $0.50 \mathrm{mmol}$ scale reaction. ${ }^{d}$ Reaction was run on $0.5 \mathrm{mmol}$ scale in $1.0 \mathrm{~mL}$ DMF. 


\section{Synthesis of Starting Materials}

\subsection{Synthesis of Aryl Triflates}

Scheme S1. Aryl triflates used in this study

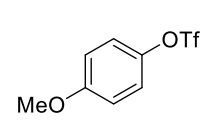

$1 \mathrm{a}$

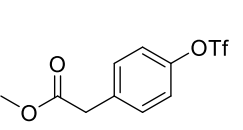

$1 \mathrm{~g}$

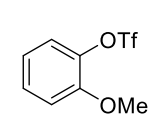

11
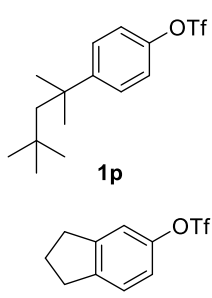

1t

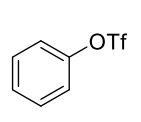

$1 b$

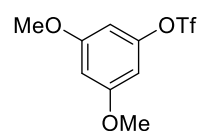

1c

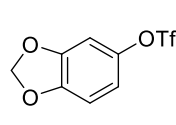

1d

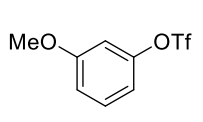

1e

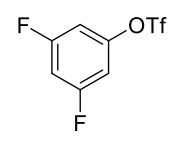

$1 f$

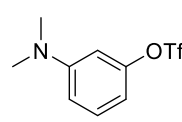

$1 \mathrm{~h}$

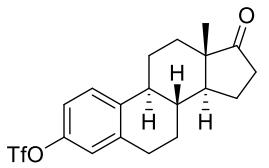

$1 \mathrm{~m}$

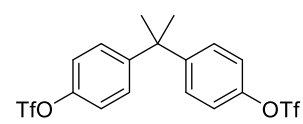

$1 q$

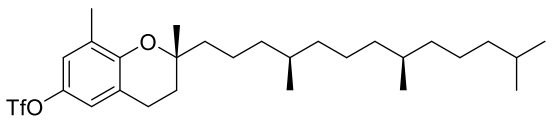

$1 \mathrm{u}$

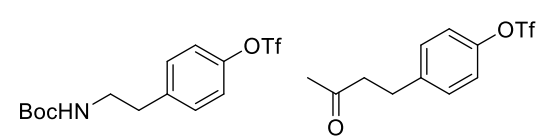

$1 \mathrm{i}$

$1 \mathrm{j}$

$1 \mathrm{k}$

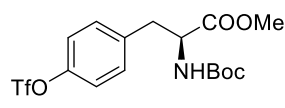

$1 n$

10

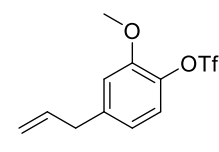

$1 \mathrm{r}$

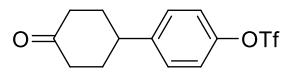

$1 \mathrm{~s}$

Known compounds $1 a^{1}, \mathbf{1 b}^{1}, \mathbf{1 c}^{2}, \mathbf{1 d}^{3}, \mathbf{1 e}^{4}, \mathbf{1 f}^{5}, \mathbf{1 g}^{6}, \mathbf{1 h}^{7}, \mathbf{1} \mathbf{j}^{8}, \mathbf{1 k}^{9}, \mathbf{1 l}^{10}, \mathbf{1 m}^{3}, \mathbf{1 n}^{3}, \mathbf{1 0}^{3}, \mathbf{1 p}^{11}, \mathbf{1 q}^{12}, \mathbf{1 r}^{13}, \mathbf{1 s}^{14}$, $\mathbf{1 t}^{15}$ and $\mathbf{1} \mathbf{u}^{1}$ were prepared according to the reported procedures.

Scheme S2. Synthesis of aryl triflate 1i

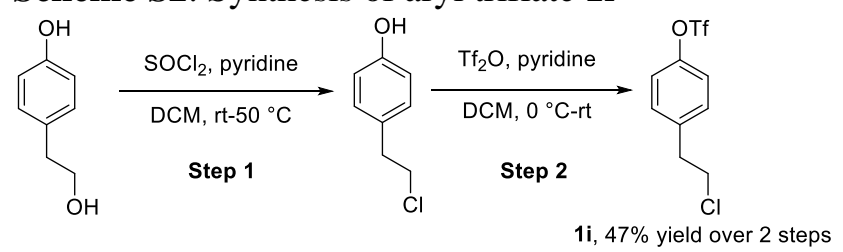

\section{Procedure for the synthesis of $1 \mathrm{i}$}

Step 1: A two-neck $100 \mathrm{~mL}$ round bottom flask equipped with a PTFE-coated stir-bar was fitted with a reflux condenser connected to a $\mathrm{N}_{2}$ Schlenk line via a needle through a rubber septum (center neck). The flask was charged with 2-(4-hydroxyphenyl)ethanol (2000 mg, $14.5 \mathrm{mmol}, 1.00$ equiv) and pyridine (5728 mg, $72.5 \mathrm{mmol}, 5.00$ equiv) through the side-neck under positive $\mathrm{N}_{2}$ flow. The reaction vessel was capped with a rubber septum and dry dichloromethane $(40 \mathrm{~mL})$ was added by syringe. The mixture was cooled in an ice-water bath for $10 \mathrm{~min}$. A solution of thionyl chloride (8627 mg, $72.5 \mathrm{mmol}, 5.00 \mathrm{equiv}$ ) in dry dichloromethane $(10 \mathrm{~mL})$ was added dropwise by syringe through the septum over $20 \mathrm{~min}$ with cooling. 
The ice-water bath was then removed. The mixture was stirred at $\mathrm{rt}$ for $2 \mathrm{~h}$, followed by heating the mixture to reflux in a $50{ }^{\circ} \mathrm{C}$ oil bath for $8 \mathrm{~h}$. When the reaction was judged complete (TLC analysis, hexanes/ethyl acetate $=5 / 1, R_{\mathrm{f}}=0.3$ ), the reaction mixture was cooled in an ice-water bath for $10 \mathrm{~min}$ before it was poured into $100 \mathrm{~mL}$ ice-cold water. The layers were separated in a $500 \mathrm{~mL}$ separatory funnel. The dichloromethane layer was set aside while the aqueous layer was washed further with dichloromethane $(50 \mathrm{~mL} \times 3)$. The combined organic layers were dried over anhydrous $\mathrm{MgSO}_{4}$, the resulting mixture was filtered to remove the $\mathrm{MgSO}_{4}$, and the filtrate was concentrated by rotary evaporation. The residue was purified by flash chromatography on silica gel (hexanes/ethyl acetate $=5 / 1$ to $3 / 1$ ). The crude 2-(4-hydroxyphenyl)ethyl chloride product was directly used in the next step without further purification.

Step 2: A two-neck $100 \mathrm{~mL}$ round bottom flask equipped with a PTFE-coated stir-bar was connected to a $\mathrm{N}_{2}$ Schlenk line via a needle through a rubber septum (center neck) and a second rubber septum was fitted to the side-neck. The flask was charged with 2-(4-hydroxyphenyl)ethyl chloride (1560 $\mathrm{mg}, 10.0 \mathrm{mmol}, 1.00$ equiv) obtained from Step 1 and pyridine (2291 mg, $29.0 \mathrm{mmol}, 2.90$ equiv) through the side-neck under positive $\mathrm{N}_{2}$ flow. The side-neck was re-capped with a rubber septum after the addition, and dry dichloromethane $(10 \mathrm{~mL})$ was added by syringe. The reaction mixture was cooled in an ice-water bath for $10 \mathrm{~min}$. A solution of trifluoromethanesulfonic anhydride ( $8178 \mathrm{mg}, 29.0 \mathrm{mmol}, 2.90$ equiv) in dry dichloromethane $(20 \mathrm{~mL})$ was added dropwise by syringe through the side-neck septum over 20 min with cooling. After the addition was complete, the ice-water bath was removed, and the reaction mixture was allowed to recover to $\mathrm{rt}$ with stirring over $12 \mathrm{~h}$. When the reaction was judged complete (TLC analysis, hexanes/ethyl acetate $=5 / 1, \mathrm{R}_{\mathrm{f}}=0.8$ ), the reaction mixture was poured into a $500 \mathrm{~mL}$ separatory funnel containing ice-cold water $(50 \mathrm{~mL})$ and dichloromethane $(50 \mathrm{~mL})$. The dichloromethane layer was set aside while the aqueous layer was washed further with dichloromethane $(50 \mathrm{~mL} \times 3)$. The combined organic layers were dried over anhydrous $\mathrm{MgSO}_{4}$, the resulting mixture was filtered to remove the $\mathrm{MgSO}_{4}$, and the filtrate was concentrated by rotary evaporation. The crude material was purified by flash chromatography on silica gel (hexane/ethyl acetate $=50 / 1$ to 10/1) to afford 4-(2-chloroethyl)phenyl trifluoromethanesulfonate (1i) $\left(1963 \mathrm{mg}, 47 \%\right.$ yield over 2 steps) as a colorless oil. ${ }^{1} \mathrm{H} \mathrm{NMR}(400 \mathrm{MHz}$, $\left.\mathrm{CDCl}_{3}\right) \delta 7.31(\mathrm{~d}, J=8.7 \mathrm{~Hz}, 2 \mathrm{H}), 7.23(\mathrm{~d}, J=8.7 \mathrm{~Hz}, 2 \mathrm{H}), 3.72(\mathrm{t}, J=7.1 \mathrm{~Hz}, 2 \mathrm{H}), 3.10(\mathrm{t}, J=7.1 \mathrm{~Hz}$, $2 \mathrm{H}) ;{ }^{13} \mathrm{C}\left\{{ }^{1} \mathrm{H}\right\} \mathrm{NMR}\left(125 \mathrm{MHz}, \mathrm{CDCl}_{3}\right) \delta 148.7,138.8,130.8,121.6,118.9$ (q, $\left.J=255.0 \mathrm{~Hz}\right), 44.6,38.4$; ${ }^{19} \mathrm{~F}\left\{{ }^{1} \mathrm{H}\right\} \mathrm{NMR}\left(376 \mathrm{MHz}, \mathrm{CDCl}_{3}\right) \delta-72.85 \mathrm{ppm}$. HRMS (ESI) $\mathrm{m} / \mathrm{z}$ calcd for $[\mathrm{M}+\mathrm{H}]^{+} 288.9908$, found 288.9904. IR $\left(\mathrm{cm}^{-1}\right): 2957,1416,1205,1135,883,608$. 


\subsection{Synthesis of Aryl Tosylates}

Scheme S3. Aryl tosylates used in this study

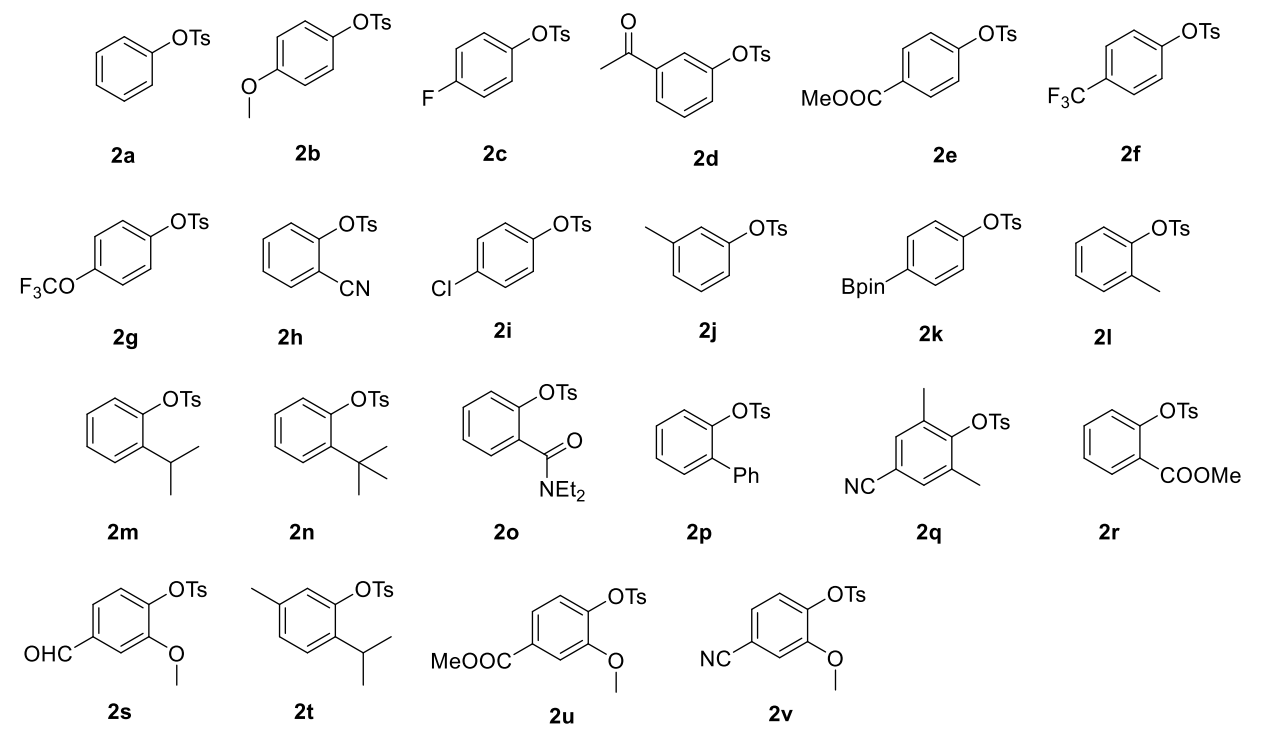

Known compounds $\mathbf{2} \mathbf{a}^{16}, \mathbf{2} \mathbf{b}^{17}, \mathbf{2} \mathbf{c}^{17}, \mathbf{2} \mathbf{d}^{18}, \mathbf{2} \mathbf{e}^{17}, \mathbf{2} \mathbf{f}^{19}, \mathbf{2} \mathbf{g}^{18}, \mathbf{2} \mathbf{h}^{20}, \mathbf{2} \mathbf{i}^{18}, \mathbf{2} \mathbf{j}^{21}, \mathbf{2} \mathbf{k}^{22}, \mathbf{2} \mathbf{l}^{17}, \mathbf{2} \mathbf{m}^{23}, \mathbf{2} \mathbf{n}^{24}, \mathbf{2} \mathbf{p}^{25}, \mathbf{2} \mathbf{q}^{22}$, $\mathbf{2} \mathbf{r}^{16}, \mathbf{2} \mathbf{s}^{26}, \mathbf{2} \mathbf{t}^{27}, \mathbf{2} \mathbf{u}^{28}$ and $\mathbf{2} \mathbf{v}^{29}$ were prepared according to reported procedures.

Scheme S4. Synthesis of aryl tosylate 20

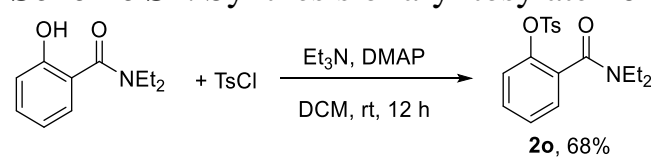

\section{Procedure for the synthesis of aryl tosylate 20}

A scintillation vial equipped with a PTFE-coated stir-bar was charged with $\mathrm{N}, \mathrm{N}$ diethylsalicylamide (965 mg, $5.00 \mathrm{mmol}, 1.00$ equiv), DMAP (61 mg, $0.50 \mathrm{mmol}, 0.10$ equiv), and triethylamine (1010 mg, $10.0 \mathrm{mmol}, 2.00$ equiv). Dry dichloromethane (10 mL) was added before the scintillation vial was capped with a rubber septum. The mixture was stirred at $\mathrm{rt}$ for $5 \mathrm{~min}$. 4-toluenesulfonyl chloride (1143 mg, $6.00 \mathrm{mmol}, 1.20$ equiv) was then added in three potions over $2 \mathrm{~min}$. The vial was recapped, and the mixture was stirred at $\mathrm{rt}$ for $12 \mathrm{~h}$. When the reaction was judged complete (TLC analysis, hexanes/ethyl acetate $=5 / 1, \mathrm{R}_{\mathrm{f}}=0.3$ ), the reaction mixture was poured into a $250 \mathrm{~mL}$ separatory funnel containing water $(30 \mathrm{~mL})$ and dichloromethane $(30 \mathrm{~mL})$. The dichloromethane layer was set aside while the aqueous layer was washed further with dichloromethane $(30 \mathrm{~mL} \times 3)$. The combined organic layers were dried over anhydrous $\mathrm{MgSO}_{4}$, the resulting mixture was filtered to remove the $\mathrm{MgSO}_{4}$, and the filtrate was concentrated by rotary evaporation. The crude material was purified by flash chromatography on silica gel (hexanes/ethyl acetate $=5 / 1$ to $3 / 1$ ) to afford 2-(diethylcarbamoyl)phenyl 4-methylbenzenesulfonate 
(2o) $\left(1180 \mathrm{mg}, 68 \%\right.$ yield) as a colorless oil. ${ }^{1} \mathrm{H} \mathrm{NMR}\left(500 \mathrm{MHz}, \mathrm{CDCl}_{3}\right) \delta 7.75(\mathrm{~d}, J=8.3 \mathrm{~Hz}, 2 \mathrm{H}), 7.29$ $(\mathrm{t}, J=9.7 \mathrm{~Hz}, 3 \mathrm{H}), 7.26-7.21(\mathrm{~m}, 3 \mathrm{H}), 3.68(\mathrm{~s}, 1 \mathrm{H}), 3.11(\mathrm{~s}, 3 \mathrm{H}), 2.40(\mathrm{~s}, 3 \mathrm{H}), 1.13$ (t, $J=7.1 \mathrm{~Hz}, 3$ $\mathrm{H}), 1.02(\mathrm{t}, J=7.1 \mathrm{~Hz}, 3 \mathrm{H}) ;{ }^{13} \mathrm{C}\left\{{ }^{1} \mathrm{H}\right\} \mathrm{NMR}\left(125 \mathrm{MHz}, \mathrm{CDCl}_{3}\right) \delta 166.4,145.7,145.5,132.9,131.4,130.2$, 130.0, 128.6, 128.4, 127.1, 122.2, 43.1, 39.1, 21.9, 14.0, 12.8 ppm. HRMS (ESI) m/z calcd for $[\mathrm{M}+\mathrm{H}]^{+}$ 348.1264, found 348.1258. IR: $\left(\mathrm{cm}^{-1}\right): 2972,2934,1623,1518,1289,1244,835,763$.

\subsection{Synthesis of Other Phenol Derivatives}

Scheme S5. Other phenol derivatives used in this study

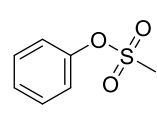

$6 a$

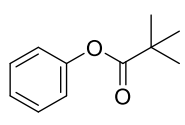

$6 b$

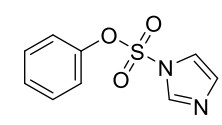

$6 c$

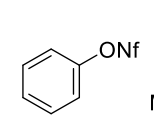

6d

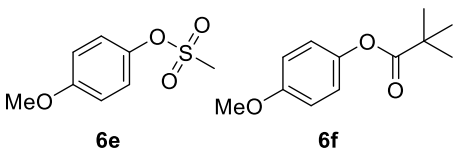

6 e

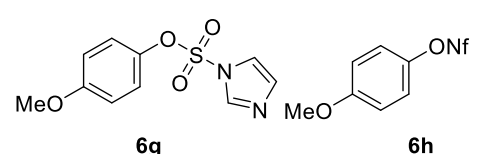

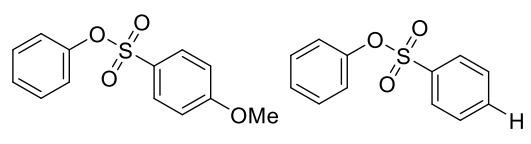

$7 b$

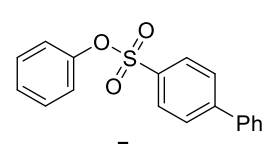

7c

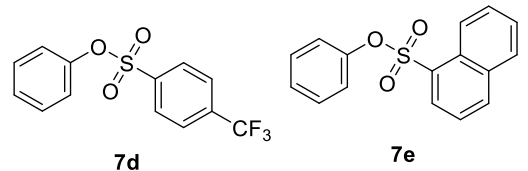

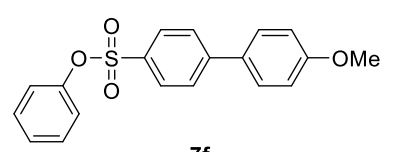

$7 f$

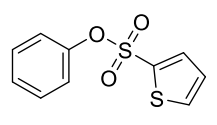

$7 g$

Known compounds $6 \mathbf{a}^{30}, 6 \mathbf{b}^{31}, \mathbf{6} \mathbf{c}^{32}, \mathbf{6} \mathbf{d}^{33}, \mathbf{6} \mathbf{e}^{30}, \mathbf{6 f}^{34}, \mathbf{6} \mathbf{g}^{32}, 6 \mathbf{h}^{35}, 7 \mathbf{a}^{36}, 7 \mathbf{b}^{37}, 7 \mathbf{d}^{38}$ and $7 \mathbf{e}^{39}$ were prepared according to reported procedures.

\section{Synthesis of phenol derivatives $7 \mathrm{c}, 7 \mathrm{f}$ and $7 \mathrm{~g}$}

Scheme S6. Synthesis of phenol derivative 7c

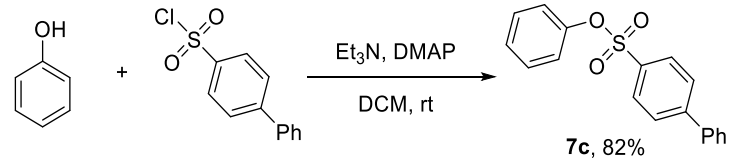

Phenyl [1,1'-biphenyl]-4-sulfonate (7c) [CAS: 20443-76-9] was synthesized following procedure for the synthesis of aryl tosylate $2 o$ (page S11) using phenol (470 mg, $5.00 \mathrm{mmol}, 1.00$ equiv), DMAP (61 mg, $0.50 \mathrm{mmol}, 0.10$ equiv), triethylamine (1010 mg, $10.0 \mathrm{mmol}, 2.00$ equiv) and 4-phenylbenzenesulfonyl chloride (1512 mg, $6.00 \mathrm{mmol}, 1.20$ equiv) as starting materials and dry dichloromethane (10 mL) as solvent. After stirring at $\mathrm{rt}$ for $12 \mathrm{~h}$, the reaction was quenched following the procedure and the crude material was purified by flash chromatography on silica gel (hexanes/ethyl acetate $=5 / 1$ to $3 / 1$ ) to afford the desired product $\left(1271 \mathrm{mg}, 82 \%\right.$ yield) as a white solid. $\mathrm{mp}=86-88{ }^{\circ} \mathrm{C} .{ }^{1} \mathrm{H} \mathrm{NMR}\left(500 \mathrm{MHz}, \mathrm{CDCl}_{3}\right.$, TMS) $\delta 7.88(\mathrm{~d}, J=8.5 \mathrm{~Hz}, 2 \mathrm{H}), 7.72(\mathrm{~d}, J=8.5 \mathrm{~Hz}, 2 \mathrm{H}), 7.61(\mathrm{~d}, J=7.1 \mathrm{~Hz}, 2 \mathrm{H}), 7.48$ (t, $J=7.4 \mathrm{~Hz}, 2$ 
H), $7.43(\mathrm{t}, J=7.3 \mathrm{~Hz}, 1 \mathrm{H}), 7.30(\mathrm{t}, J=7.5 \mathrm{~Hz}, 2 \mathrm{H}), 7.25(\mathrm{t}, J=7.3 \mathrm{~Hz}, 1 \mathrm{H}), 7.03(\mathrm{~d}, J=7.7 \mathrm{~Hz}, 2 \mathrm{H})$; ${ }^{13} \mathrm{C}\left\{{ }^{1} \mathrm{H}\right\} \mathrm{NMR}\left(100 \mathrm{MHz}, \mathrm{CDCl}_{3}\right) \delta 149.8,147.2,138.9,134.0,129.8,129.3,129.2,129.0,127.8,127.5$, 127.3, $122.5 \mathrm{ppm}$. HRMS (ESI) $\mathrm{m} / \mathrm{z}$ calcd for $[\mathrm{M}+\mathrm{Na}]^{+} 333.0556$, found 333.0552. IR $\left(\mathrm{cm}^{-1}\right): 3062,1593$, 1483, 1349, 1182, 852, 762, 681.

Scheme S7. Synthesis of phenol derivative $7 \mathbf{f}$

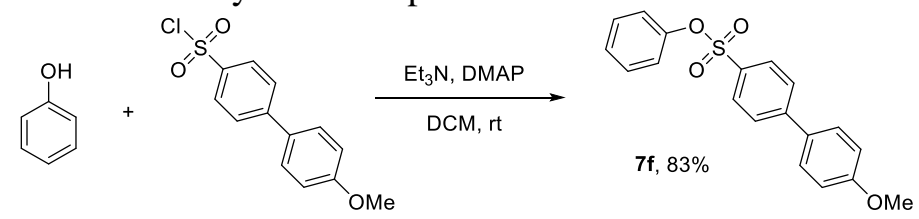

Phenyl 4'-methoxy-[1,1'-biphenyl]-4-sulfonate (7f) was synthesized following procedure for the synthesis of aryl tosylate $2 o$ (page S11) using phenol (282 mg, $3.00 \mathrm{mmol}, 1.00$ equiv), DMAP (37 mg, $0.30 \mathrm{mmol}, 0.10$ equiv), triethylamine (606 mg, $6.00 \mathrm{mmol}, 2.00$ equiv) and 4'-methoxybiphenyl-4sulfonyl chloride (1015 mg, $3.60 \mathrm{mmol}, 1.20$ equiv) as starting materials and dry dichloromethane (6 mL) as solvent. After stirring at $\mathrm{rt}$ for $12 \mathrm{~h}$, the reaction was quenched following the procedure and the crude material was purified by flash chromatography on silica gel (hexanes/ethyl acetate $=5 / 1$ to $3 / 1$ ) to afford the desired product $(850 \mathrm{mg}, 83 \%$ yield $)$ as a white solid. $\mathrm{mp}=100-102{ }^{\circ} \mathrm{C} .{ }^{1} \mathrm{H} \mathrm{NMR}\left(500 \mathrm{MHz}, \mathrm{CDCl}_{3}\right.$, TMS) $\delta 7.85(\mathrm{~d}, J=8.6 \mathrm{~Hz}, 2 \mathrm{H}), 7.68(\mathrm{~d}, J=8.5 \mathrm{~Hz}, 2 \mathrm{H}), 7.57$ (d, $J=8.7 \mathrm{~Hz}, 2 \mathrm{H}), 7.30$ (t, $J=7.5 \mathrm{~Hz}, 2$ $\mathrm{H}), 7.28-7.24(\mathrm{~m}, 1 \mathrm{H}), 7.02(\mathrm{~m}, 4 \mathrm{H}), 3.87(\mathrm{~s}, 3 \mathrm{H}) ;{ }^{13} \mathrm{C}\left\{{ }^{1} \mathrm{H}\right\} \mathrm{NMR}\left(125 \mathrm{MHz}, \mathrm{CDCl}_{3}\right) \delta 160.5,149.8$, $146.8,133.1,131.2,129.8,129.2,128.7,127.3,127.1,122.6,114.7,55.6$ ppm. HRMS (ESI) m/z calcd for $[\mathrm{M}+\mathrm{H}]^{+}$341.0842, found 341.0841. IR $\left(\mathrm{cm}^{-1}\right): 3069,2839,1594,1521,1372,1178,860,782,634$.

Scheme S8. Synthesis of phenol derivative $7 \mathbf{g}$

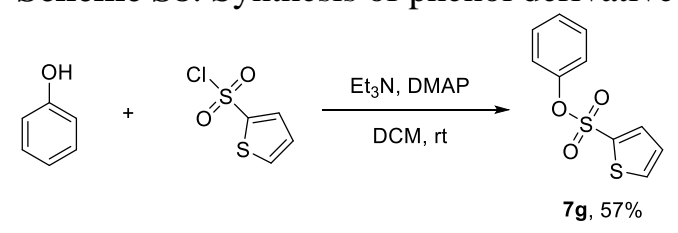

Phenyl thiophene-2-sulfonate (7g) was synthesized following procedure for the synthesis of aryl tosylate $2 o$ (page S11) using phenol (282 mg, $3.00 \mathrm{mmol}, 1.00$ equiv), DMAP (37 mg, $0.30 \mathrm{mmol}, 0.10$ equiv), triethylamine (606 mg, $6.00 \mathrm{mmol}, 2.00$ equiv) and 2-thiophenesulfonyl chloride (655 mg, $3.60 \mathrm{mmol}, 1.2$ equiv) as starting materials and dry dichloromethane $(6 \mathrm{~mL})$ as solvent. After stirring at $\mathrm{rt}$ for $12 \mathrm{~h}$, the reaction was quenched following the procedure and the crude material was purified by flash chromatography on silica gel (hexanes/ethyl acetate $=5 / 1$ ) to afford the desired product $(410 \mathrm{mg}, 57 \%$ yield) as a colorless oil. ${ }^{1} \mathrm{H}$ NMR $\left(500 \mathrm{MHz}, \mathrm{CDCl}_{3}\right.$, TMS) $\delta 7.72(\mathrm{dd}, J=5.0,1.3 \mathrm{~Hz}, 1 \mathrm{H}), 7.57(\mathrm{dd}, J=$ $3.8,1.3 \mathrm{~Hz}, 1 \mathrm{H}), 7.35-7.25(\mathrm{~m}, 3 \mathrm{H}), 7.10(\mathrm{dd}, J=5.0,3.8 \mathrm{~Hz}, 1 \mathrm{H}), 7.04(\mathrm{~d}, J=8.1 \mathrm{~Hz}, 2 \mathrm{H}) ;{ }^{13} \mathrm{C}\left\{{ }^{1} \mathrm{H}\right\}$ 
NMR $\left(125 \mathrm{MHz}, \mathrm{CDCl}_{3}\right) \delta 149.7,135.7,134.8,134.6,129.9,127.7,127.6,122.4 \mathrm{ppm} . \mathrm{HRMS}(\mathrm{ESI}) \mathrm{m} / \mathrm{z}$ calcd for $[\mathrm{M}+\mathrm{Na}]^{+}$262.9807, found 262.9806. IR $\left(\mathrm{cm}^{-1}\right): 3099,1486,1375,1192,1144,861,778,728$, 585. 


\subsection{Availability Analysis of Selected Phenols and Their Aryl Halides (Halide = Cl, Br, I) Counterparts}

Phenols (Table $\mathrm{S} 2, \mathrm{X}=\mathrm{OH}$ ) can be a very convenient aryl source when their aryl halide counterparts $(\mathrm{X}=\mathrm{Cl}, \mathrm{Br}, \mathrm{I})$ are significantly more costly or not commercially available. Here we listed the detailed analysis of prices (US\$ per gram as well as US\$ per mol) for selected phenols (Scheme 2, examples where $\mathrm{Ar}-\mathrm{OSO}_{2} \mathrm{R}$ is more convenient than $\mathrm{Ar}-\mathrm{X}$ ) and their aryl halides counterparts (Table S2, entries 114). We also provided the availability analysis of other phenols we used as starting materials in Scheme 2 that are natural products, bioactive compounds, or precursors to important molecules (Table S2, entries 1530). In many cases there is a wide variation in pricing or quantity available between different vendors. Therefore, for each compound we listed prices from at least 2 vendors (if there are 2 or more vendors). In each entry, compounds shaded in blue indicate the most commercially available variants of the molecules with the same substitution pattern. All the data provided here were from SciFinder search results for compounds' commercial sources on February 2nd, 2020.

Table S2. Availability analysis of selected phenols and their aryl halides $(\mathrm{X}=\mathrm{Cl}, \mathrm{Br}, \mathrm{I})$ counterparts.

\begin{tabular}{|c|c|c|c|c|c|}
\hline entry & $\begin{array}{l}\text { compound structures } \\
\text { and natural sources or } \\
\text { applications when } \mathrm{X}= \\
\mathrm{OH}\end{array}$ & $\mathrm{X}=\mathrm{OH}$ & $\mathrm{X}=\mathrm{Cl}$ & $\mathrm{X}=\mathrm{Br}$ & $X=I$ \\
\hline 1 & $\begin{array}{c}\mathrm{CN}_{\mathrm{CN}} \\
\text { etravirine (Tibotec) } \\
\text { precursor }\end{array}$ & $\begin{array}{l}\text { CAS: 4198-90-7 } \\
\text { Combi-Blocks } \\
(\$ 10 / 5 \mathrm{~g}, \$ 290 / \mathrm{mol}) \\
\text { AA Blocks }(\$ 6 / 5 \mathrm{~g}, \\
\$ 170 / \mathrm{mol}) \\
\text { Ambeed, Inc. }(\$ 10 / 5 \mathrm{~g}, \\
\$ 290 / \mathrm{mol})\end{array}$ & $\begin{array}{l}\text { CAS: 1097629-24-7 } \\
\text { HE Chemical, China } \\
\text { (\$7998/1g, } \\
\$ 1,320,000 / \mathrm{mol}) \\
\text { Aquila Pharmatech } \\
\text { (Synthesis on demand) }\end{array}$ & $\begin{array}{l}\text { CAS: } 75344-77-3 \\
\text { Ambeed, Inc. }(\$ 63 / 5 \mathrm{~g}, \\
\$ 2,600 \mathrm{~mol}) \\
\text { Combi-Blocks } \\
\left(\begin{array}{l}\$ 80 / 5 \mathrm{~g}, \$ 3,300 / \mathrm{mol}) \\
\text { Synthonix }(\$ 80 / 5 \mathrm{~g}, \\
\$ 3,300 / \mathrm{mol})\end{array}\right.\end{array}$ & $\begin{array}{l}\text { CAS: 127311-09-2 } \\
\text { AA Blocks }(\$ 32 / 100 \\
\text { mg, } \$ 8,200 / \mathrm{mol}) \\
\text { Ambeed, Inc. }(\$ 81 / \mathrm{g}, \\
\quad \$ 21,000 / \mathrm{mol}) \\
\text { Matrix Scientific } \\
\text { (\$742/250 mg, } \\
\$ 762,000 / \mathrm{mol})\end{array}$ \\
\hline 2 & frambinone & $\begin{array}{c}\text { CAS: } 5471-51-2 \\
\text { Alfa Aeasr }(\$ 41 / 25 \mathrm{~g}, \\
\$ 270 / \mathrm{mol}) \\
\text { Ambeed, Inc }(\$ 6 / 25 \mathrm{~g} \text {, } \\
\$ 40 / \mathrm{mol}) \\
\text { Santa Cruz } \\
\text { Biotechnology } \\
(\$ 121 / 1 \mathrm{~kg}, \$ 20 / \mathrm{mol})\end{array}$ & $\begin{array}{c}\text { CAS: 3506-75-0 } \\
\text { AA Blocks }(\$ 200 / 250 \\
\mathrm{mg}, \$ 146,000 / \mathrm{mol}) \\
\text { Ambeed, Inc } \\
(\$ 250 / \mathrm{g}, \$ 46,000 / \mathrm{mol}) \\
\text { Matirx Scientific } \\
(\$ 160 / 10 \mathrm{~g}, \\
\$ 2,900 / \mathrm{mol})\end{array}$ & $\begin{array}{c}\text { CAS: } 89201-84-3 \\
\text { Ambeed, Inc } \\
\text { (\$250/g, \$56,000/mol) } \\
\text { Matirx Scientific } \\
\text { (\$90/5g, \$4,100/mol) } \\
\text { AURUM Pharmatech } \\
\text { (\$284/250 mg, } \\
\$ 314,000 / \mathrm{mol})\end{array}$ & $\begin{array}{l}\text { CAS: 918540-55-3 } \\
\text { AA Blocks }(\$ 1172 / \mathrm{g}, \\
\$ 321,000 / \mathrm{mol}) \\
\text { Ambeed, Inc }(\$ 300 / \mathrm{g} \text {, } \\
\$ 82,000 / \mathrm{mol}) \\
\text { AURUM Pharmatech } \\
\text { (\$806/g, } \\
\$ 221,000 / \mathrm{mol})\end{array}$ \\
\hline
\end{tabular}




\begin{tabular}{|c|c|c|c|c|c|}
\hline 3 & estrone & $\begin{array}{c}\text { CAS: 53-16-7 } \\
\text { AA Blocks ( } \$ 10 / \mathrm{g}, \\
\$ 2,700 / \mathrm{mol}) \\
\text { Combi-Blocks }(\$ 10 / \mathrm{g}, \\
\$ 2,700 / \mathrm{mol}) \\
\text { Santa Cruz } \\
\text { Biotechnology }(\$ 31 / \mathrm{g}, \\
\$ 8,400 / \mathrm{mol})\end{array}$ & $\begin{array}{l}\text { CAS: 5764-23-8 } \\
\text { Only } 1 \text { vendor, no } \\
\text { price provided }\end{array}$ & $\begin{array}{l}\text { CAS: 1394922-25-8 } \\
\text { Not commercially } \\
\quad \text { available }\end{array}$ & $\begin{array}{l}\text { CAS: } 1243197-32-1 \\
\text { Not commercially } \\
\text { available }\end{array}$ \\
\hline 4 & $L$ & $\begin{array}{l}\text { CAS: } 4326-36-7 \\
\text { AA Blocks }(\$ 8 / 5 \mathrm{~g}, \\
\$ 470 / \mathrm{mol}) \\
\text { Ambeed, Inc }(\$ 7 / 5 \mathrm{~g}, \\
\$ 410 / \mathrm{mol}) \\
\text { Combi-Blocks } \\
(\$ 11 / 5 \mathrm{~g}, \$ 650 / \mathrm{mol})\end{array}$ & $\begin{array}{l}\text { CAS: } 172035-26-6 \\
\text { Only } 3 \text { vendors, no } \\
\text { prices provided }\end{array}$ & $\begin{array}{c}\text { CAS: 266306-18-7 } \\
\text { AA Blocks (\$267/g, } \\
\$ 95,000 / \mathrm{mol}) \\
\text { Ambeed, Inc }(\$ 222 / \mathrm{g}, \\
\$ 79,000 / \mathrm{mol}) \\
\text { Combi-Blocks } \\
(\$ 240 / \mathrm{g}, \$ 85,000 / \mathrm{mol})\end{array}$ & $\begin{array}{c}\text { CAS: } 113850-76-3 \\
\text { AA Blocks }(\$ 178 / \mathrm{g}, \\
\$ 71,000 / \mathrm{mol}) \\
\text { Combi-Blocks } \\
(\$ 160 / \mathrm{g}, \$ 64,000 / \mathrm{mol}) \\
\text { AURUM Pharmatech } \\
(\$ 223 / \mathrm{g}, \$ 89,000 / \mathrm{mol})\end{array}$ \\
\hline 5 & ketoconazole precursor & $\begin{array}{l}\text { CAS: } 67914-60-7 \\
\text { AA Blocks }(\$ 10 / 5 \mathrm{~g}, \\
\$ 440 / \mathrm{mol}) \\
\text { Alfa Aesar }(\$ 29 / 25 \mathrm{~g}, \\
\$ 250 / \mathrm{mol}) \\
\text { Combi-Blocks } \\
(\$ 25 / 25 \mathrm{~g}, \$ 220 / \mathrm{mol})\end{array}$ & $\begin{array}{c}\text { CAS: 150557-99-6 } \\
\text { AA Blocks }(\$ 138 / 100 \\
\text { mg, } \$ 328,000 / \mathrm{mol}) \\
\text { AURUM Pharmatech } \\
(\$ 869 / 5 \mathrm{~g}, \\
\$ 41,000 / \mathrm{mol})\end{array}$ & $\begin{array}{l}\text { CAS: 678996-43-5 } \\
\text { AA Blocks }(\$ 223 / \mathrm{g}, \\
\$ 63,000 / \mathrm{mol}) \\
\text { Combi-Blocks } \\
(\$ 200 / \mathrm{g}, \$ 56,000 / \mathrm{mol})\end{array}$ & $\begin{array}{l}\text { CAS: 1197293-73-4 } \\
\text { Only } 1 \text { vendor, no } \\
\text { prices provided }\end{array}$ \\
\hline 6 & $\begin{array}{c}\text { pleconaril (Picovir) } \\
\text { precursor }\end{array}$ & $\begin{array}{c}\text { CAS: 4421-08-3 } \\
\text { AA Blocks } \\
(\$ 227 / 500 \mathrm{~g}, \$ 68 / \mathrm{mol}) \\
\text { Alfa Aesar } \\
(\$ 159 / 100 \mathrm{~g}, \\
\$ 240 / \mathrm{mol}) \\
\text { Combi-Blocks } \\
(\$ 240 / 500 \mathrm{~g}, \$ 72 / \mathrm{mol})\end{array}$ & $\begin{array}{l}\text { CAS: } 189620-40-8 \\
\text { AA Blocks }(\$ 125 / \mathrm{g}, \\
\$ 21,000 / \mathrm{mol}) \\
\text { Matrix Scientific } \\
(\$ 622 / 5 \mathrm{~g}, \\
\$ 21,000 / \mathrm{mmol}) \\
\text { Combi-Blocks } \\
(\$ 125 / \mathrm{g}, \$ 21,000 / \mathrm{mol})\end{array}$ & 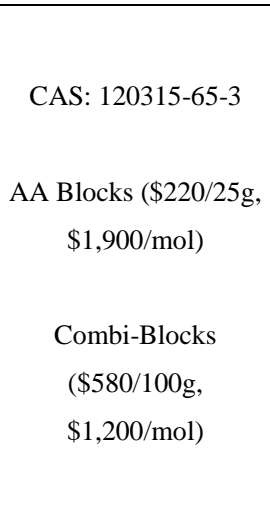 & $\begin{array}{l}\text { CAS: 652997-56-3 } \\
\text { Alichem product } \\
(\$ 677 / \mathrm{g}, 175,000 / \mathrm{mol}) \\
\text { FCH group reagents } \\
\text { for synthesis }(\$ 571 / \mathrm{g} \text {, } \\
148,000 / \mathrm{mol})\end{array}$ \\
\hline
\end{tabular}




\begin{tabular}{|c|c|c|c|c|c|}
\hline 7 & 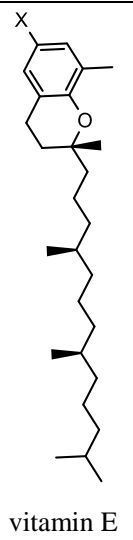 & $\begin{array}{l}\text { CAS: 119-13-1 } \\
\text { Santa Cruz } \\
\text { Biotechnology } \\
(\$ 562 / 100 \mathrm{~g}, \\
\$ 2,200 / \mathrm{mol}) \\
\text { Sigma-Aldrich } \\
\text { (\$629/100 g, } \\
\$ 2,500 / \mathrm{mol})\end{array}$ & $\begin{array}{l}\text { CAS: } 1247868-59-2 \\
\text { Not commercially } \\
\text { available }\end{array}$ & $\begin{array}{l}\text { CAS: } 1247868-54-7 \\
\text { Not commercially } \\
\quad \text { available }\end{array}$ & $\begin{array}{l}\text { CAS: 2242797-94-8 } \\
\text { Not commercially } \\
\text { available }\end{array}$ \\
\hline 8 & & $\begin{array}{c}\text { CAS: 121-33-5 } \\
\text { AA Blocks }(\$ 83 / 500 \mathrm{~g}, \\
\$ 25 / \mathrm{mol}) \\
\text { Alfa Aesar }(\$ 63 / 500 \mathrm{~g}, \\
\$ 19 / \mathrm{mol}) \\
\text { Oakwood Chemical } \\
(\$ 55 / 500 \mathrm{~g}, \$ 16 / \mathrm{mol})\end{array}$ & $\begin{array}{l}\text { CAS: 13726-16-4 } \\
\text { AA Blocks (\$13/g, } \\
\$ 2,200 / \mathrm{mol}) \\
\text { Combi-Blocks }(\$ 12 / \mathrm{g}, \\
\$ 2,100 / \mathrm{mol}) \\
\text { Oakwood Chemical } \\
\text { (\$18/g, } \$ 3,000 / \mathrm{mmol})\end{array}$ & $\begin{array}{c}\text { CAS: 43192-34-3 } \\
\text { AA Blocks (\$31/g, } \\
\text { \$6,600/mol) } \\
\text { Combi-Blocks ( } \$ 35 / \mathrm{g}, \\
\$ 7,500 / \mathrm{mol}) \\
\text { Matrix Scientific } \\
(\$ 306 / \mathrm{g}, \$ 66,000 / \mathrm{mol})\end{array}$ & $\begin{array}{c}\text { CAS: } 121404-83-9 \\
\text { AA Blocks }(\$ 217 / 250 \\
\mathrm{mg}, \$ 227,000 / \mathrm{mol}) \\
\text { Combi-Blocks } \\
(\$ 230 / 250 \mathrm{mg}, \\
\$ 240,000 / \mathrm{mol})\end{array}$ \\
\hline 9 & & $\begin{array}{c}\text { CAS: } 97-53-0 \\
\text { AA Blocks }(\$ 27 / 5 \mathrm{~g}, \\
\$ 880 / \mathrm{mol}) \\
\text { Alfa Aesar }(\$ 33 / 100 \mathrm{~g}, \\
\$ 54 / \mathrm{mol}) \\
\text { Oakwood Chemical } \\
(\$ 55 / 500 \mathrm{~g}, \$ 18 / \mathrm{mol})\end{array}$ & $\begin{array}{l}\text { CAS: } 1256467-57-8 \\
\text { Rieke Metals }(\$ 566 / \mathrm{g} \text {, } \\
\qquad \$ 103,000 / \mathrm{mmol})\end{array}$ & $\begin{array}{l}\text { No CAS number } \\
\text { Not commercially } \\
\text { available }\end{array}$ & $\begin{array}{l}\text { No CAS number } \\
\text { Not commercially } \\
\text { available }\end{array}$ \\
\hline 10 & thymol & $\begin{array}{l}\text { CAS: } 89-83-8 \\
\text { AA Blocks }(\$ 96 / 100 \mathrm{~g}, \\
\$ 140 / \mathrm{mol}) \\
\text { Alfa Aesar }(\$ 75 / 500 \mathrm{~g}, \\
\$ 22 / \mathrm{mol}) \\
\text { Oakwood Chemical } \\
(\$ 48 / 500 \mathrm{~g}, \$ 14 / \mathrm{mol})\end{array}$ & $\begin{array}{l}\text { CAS: 4395-80-6 } \\
\text { Only } 6 \text { vendors, no } \\
\text { prices provided. }\end{array}$ & $\begin{array}{l}\text { CAS: } 4478-10-8 \\
\text { Sigma-Aldrich } \\
\quad(\$ 139 / \mathrm{g}, \\
\$ 29,000 / \mathrm{mmol})\end{array}$ & $\begin{array}{l}\text { CAS: 4395-81-7 } \\
\text { Only } 6 \text { vendors, no } \\
\text { prices provided. }\end{array}$ \\
\hline
\end{tabular}




\begin{tabular}{|c|c|c|c|c|c|}
\hline 11 & $\begin{array}{l}\qquad \prod_{0} \\
\text { precursor to liquid } \\
\text { crystals after } \\
\text { olefination and } \\
\text { reduction }\end{array}$ & $\begin{array}{l}\text { CAS: } 105640-07-1 \\
\text { AA Blocks } \\
(\$ 177 / 100 \mathrm{~g}, \\
\$ 340 / \mathrm{mol}) \\
\text { Ambeed, Inc } \\
(\$ 142 / 100 \mathrm{~g}, \\
\$ 270 / \mathrm{mol}) \\
\text { TCI America } \\
(\$ 91 / 25 \mathrm{~g}, \$ 690 / \mathrm{mol})\end{array}$ & $\begin{array}{l}\text { CAS: } 14472-80-1 \\
\text { AA Blocks (\$280/5g, } \\
\$ 11,600 / \mathrm{mol}) \\
\text { Ambeed, Inc } \\
(\$ 990 / 25 \mathrm{~g}, \\
\$ 8,230 / \mathrm{mol}) \\
\text { Combi-Blocks } \\
(\$ 165 / \mathrm{g}, \$ 34,000 / \mathrm{mol})\end{array}$ & $\begin{array}{l}\text { CAS: } 84892-43-3 \\
\text { Matrix Scientific } \\
(\$ 2400 / 10 \mathrm{~g} \\
\$ 60,000 / \mathrm{mol}) \\
\text { AA Blocks } \\
(\$ 51 / 250 \mathrm{mg}, \\
\$ 51,000 / \mathrm{mol})\end{array}$ & $\begin{array}{l}\text { CAS: } 1449768-19-7 \\
\text { Not commercially } \\
\text { available }\end{array}$ \\
\hline 12 & $\begin{array}{l}\text { precursor to } \\
\text { carindacillin and } \\
\text { candoxatril }\end{array}$ & $\begin{array}{l}\text { CAS: 1470-94-6 } \\
\text { AA Blocks } \\
(\$ 185 / 100 \mathrm{~g}, \\
\$ 250 / \mathrm{mol}) \\
\text { Oakwood Chemical } \\
(\$ 15 / 5 \mathrm{~g}, \$ 400 / \mathrm{mol}) \\
\\
\text { TCI America } \\
(\$ 114 / 100 \mathrm{~g}, \\
\$ 150 / \mathrm{mol})\end{array}$ & $\begin{array}{l}\text { CAS: 60669-08-1 } \\
\text { Matrix Scientific } \\
(\$ 1165 / \mathrm{g}, \\
\$ 177,000 / \mathrm{mol}) \\
\text { Sigma-Aldrich } \\
(\$ 404 / \mathrm{g}, \$ 61,000 / \mathrm{mol})\end{array}$ & $\begin{array}{l}\text { CAS: 6134-54-9 } \\
\text { AA Blocks } \\
(\$ 106 / 100 \mathrm{mg}, \\
\$ 208,000 / \mathrm{mol}) \\
\\
\text { Sigma-Aldrich } \\
(\$ 39.9 / 100 \mathrm{mg}, \\
\$ 78,000 / \mathrm{mol})\end{array}$ & $\begin{array}{l}\text { CAS: 132464-83-6 } \\
\text { AA Blocks }(\$ 256 / \mathrm{g}, \\
\$ 62,000 / \mathrm{mol}) \\
\text { Matrix Scientific } \\
(\$ 249 / \mathrm{g}, \$ 60,000 / \mathrm{mol})\end{array}$ \\
\hline 13 & $\begin{array}{l}\text { MeOOC } \\
\text { from vanillic acid }\end{array}$ & $\begin{array}{c}\text { CAS: } 3943-74-6 \\
\text { AA Blocks }(\$ 68 / 100 \mathrm{~g}, \\
\$ 120 / \mathrm{mol}) \\
\text { Ambeed, Inc } \\
\left(\begin{array}{l}\$ 216 / 500 \mathrm{~g}, \$ 76 / \mathrm{mol}) \\
\text { Combi-Blocks }\end{array}\right. \\
(\$ 225 / 500 \mathrm{~g}, \$ 82 / \mathrm{mol})\end{array}$ & $\begin{array}{l}\text { CAS: 116022-18-5 } \\
\qquad \begin{array}{l}\text { AA Blocks } \\
(\$ 682 / 100 \mathrm{~g} \\
\$ 1,360 / \mathrm{mol}) \\
\text { Combi-Blocks } \\
(\$ 600 / 100 \mathrm{~g} \\
\$ 1,200 / \mathrm{mmol})\end{array}\end{array}$ & $\begin{array}{l}\text { CAS: 17100-63-9 } \\
\begin{array}{l}\text { AA Blocks } \\
(\$ 589 / 100 \mathrm{~g}, \\
\$ 1,430 / \mathrm{mol}) \\
\text { Combi-Blocks } \\
(\$ 530 / 100 \mathrm{~g} \\
\$ 1,290 / \mathrm{mol})\end{array}\end{array}$ & $\begin{array}{l}\text { CAS: } 35387-92-9 \\
\begin{array}{c}\text { AA Blocks } \\
(\$ 2000 / 100 \mathrm{~g}, \\
\$ 5,840 / \mathrm{mol}) \\
\text { Combi-Blocks } \\
(\$ 720 / 25 \mathrm{~g}, \\
\$ 8,400 / \mathrm{mol})\end{array}\end{array}$ \\
\hline 14 & $\begin{array}{l}\text { from 4-tert- } \\
\text { octylphenol, a } \\
\text { component of resins } \\
\text { and paints }\end{array}$ & $\begin{array}{c}\text { CAS: } 140-66-9 \\
\text { Ambeed, Inc } \\
(\$ 17 / 25 \mathrm{~g}, \$ 140 / \mathrm{mol}) \\
\text { Oakwood Chemical } \\
(\$ 20 / 100 \mathrm{~g}, \$ 41 / \mathrm{mol}) \\
\text { TCI America } \\
(\$ 32 / 500 \mathrm{~g}, \$ 13 / \mathrm{mol})\end{array}$ & $\begin{array}{l}\text { No CAS number } \\
\text { Not commercially } \\
\text { available }\end{array}$ & $\begin{array}{l}\text { CAS: } 34549-13-8 \\
\text { Not commercially } \\
\text { available }\end{array}$ & $\begin{array}{l}\text { No CAS number } \\
\text { Not commercially } \\
\text { available }\end{array}$ \\
\hline
\end{tabular}




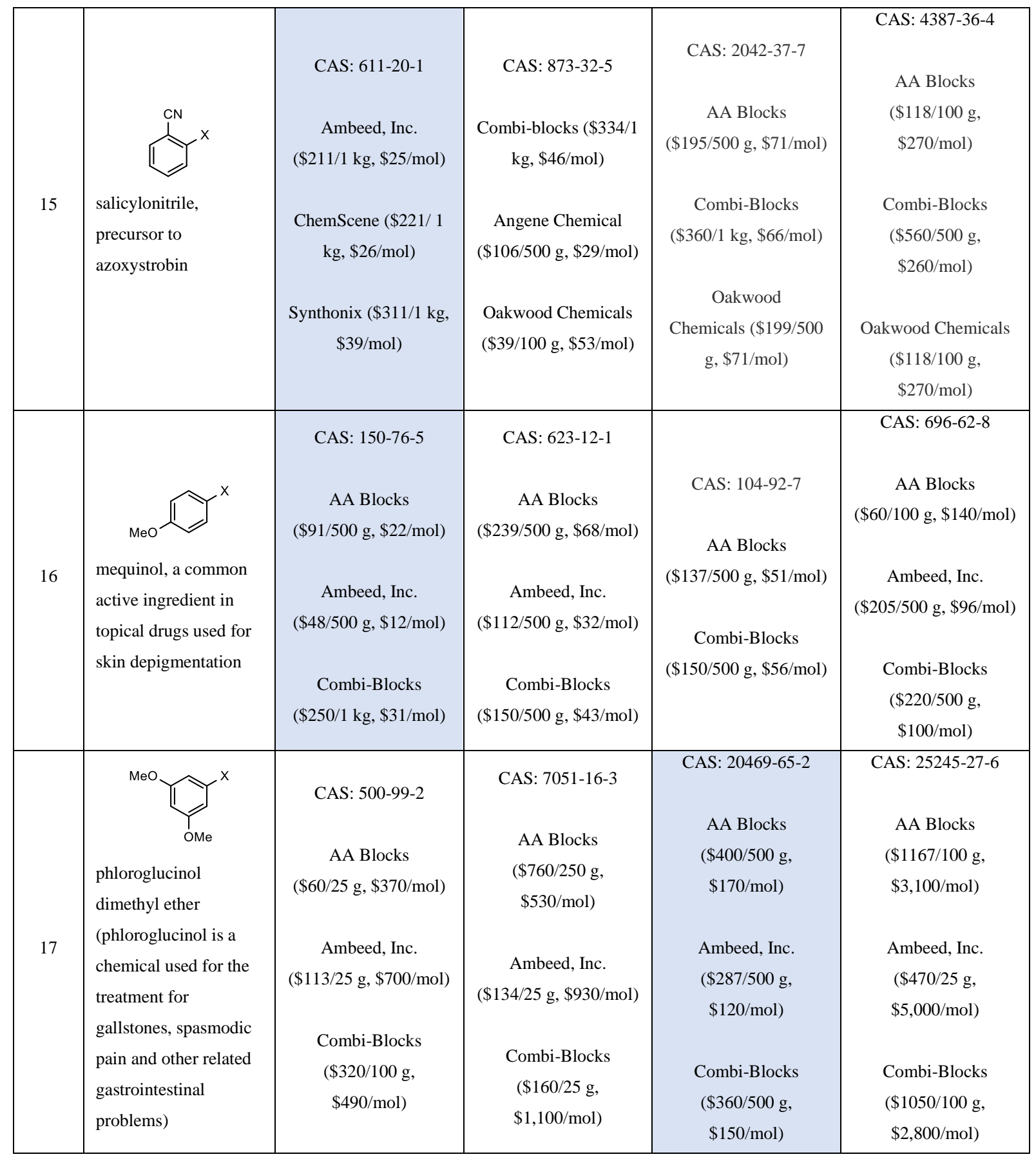




\begin{tabular}{|c|c|c|c|c|c|}
\hline 18 & $\begin{array}{l}\text { precursor to cisapride, } \\
\text { a drug that increases } \\
\text { motility in the upper } \\
\text { gastrointestinal tract }\end{array}$ & $\begin{array}{c}\text { CAS: } 371-41-5 \\
\text { AA Blocks } \\
(\$ 110 / 500 \mathrm{~g}, \$ 25 / \mathrm{mol}) \\
\text { Ambeed, Inc. } \\
(\$ 199 / 1 \mathrm{~kg}, \$ 22 / \mathrm{mol}) \\
\text { Oakwood } \\
\text { Chemicals } \\
(\$ 85 / 500 \mathrm{~g}, \$ 19 / \mathrm{mol})\end{array}$ & $\begin{array}{c}\text { CAS: 352-33-0 } \\
\text { AA Blocks } \\
(\$ 15 / 25 \mathrm{~g}, \\
\$ 78 / \mathrm{mol}) \\
\text { Oakwood } \\
\text { Chemicals } \\
(\$ 145 / 500 \mathrm{~g}, \$ 38 / \mathrm{mol}) \\
\\
\text { Matrix Scientific } \\
(\$ 125 / 500 \mathrm{~g}, \\
\$ 33 / \mathrm{mol})\end{array}$ & $\begin{array}{c}\text { CAS: } 460-00-4 \\
\text { AA Blocks } \\
(\$ 88 / 500 \mathrm{~g}, \$ 31 / \mathrm{mol}) \\
\text { Ambeed, Inc. } \\
(\$ 13 / 25 \mathrm{~g}, \$ 91 / \mathrm{mol}) \\
\text { Combi-Blocks } \\
(\$ 40 / 100 \mathrm{~g}, \$ 70 / \mathrm{mol})\end{array}$ & $\begin{array}{l}\text { CAS: } 352-34-1 \\
\text { AA Blocks } \\
(\$ 283 / 500 \mathrm{~g}, \\
\$ 120 / \mathrm{mol}) \\
\text { Ambeed, Inc. } \\
(\$ 142 / 500 \mathrm{~g}, \$ 63 / \mathrm{mol}) \\
\text { Combi-Blocks } \\
(\$ 320 / 1 \mathrm{~kg}, \$ 71 / \mathrm{mol})\end{array}$ \\
\hline 19 & $\begin{array}{l}o \text {-Cresol, one of the } \\
\text { chemical compounds } \\
\text { found in castoreum, } \\
\text { widely used in the } \\
\text { production of other } \\
\text { chemicals }\end{array}$ & $\begin{array}{c}\text { CAS: } 95-48-7 \\
\text { AA Blocks } \\
(\$ 61 / 500 \mathrm{~g}, \$ 13 / \mathrm{mol}) \\
\text { Oakwood Chemicals } \\
(\$ 60 / 500 \mathrm{~g}, \$ 13 / \mathrm{mol})\end{array}$ & $\begin{array}{c}\text { CAS: 95-49-8 } \\
\text { AA Blocks } \\
(\$ 75 / 100 \mathrm{~g}, \$ 94 / \mathrm{mol}) \\
\text { Oakwood Chemicals } \\
(\$ 19 / 100 \mathrm{~g}, \$ 24 / \mathrm{mol}) \\
\text { Matrix Scientific } \\
(\$ 84 / 100 \mathrm{~g}, \\
\$ 110 / \mathrm{mol})\end{array}$ & $\begin{array}{c}\text { CAS: } 95-46-5 \\
\text { AA Blocks } \\
(\$ 283 / 500 \mathrm{~g}, \$ 97 / \mathrm{mol}) \\
\text { Combi-Blocks } \\
(\$ 320 / 1 \mathrm{~kg}, \$ 56 / \mathrm{mol}) \\
\text { Oakwood Chemicals } \\
(\$ 178 / 500 \mathrm{~g}, \$ 61 / \mathrm{mol})\end{array}$ & $\begin{array}{c}\text { CAS: } 615-37-2 \\
\text { AA Blocks } \\
(\$ 50 / 100 \mathrm{~g}, \$ 110 / \mathrm{mol}) \\
\text { Ambeed, Inc. } \\
(\$ 172 / 500 \mathrm{~g}, \$ 75 / \mathrm{mol}) \\
\text { Combi-Blocks } \\
(\$ 400 / 1 \mathrm{~kg}, \$ 87 / \mathrm{mol})\end{array}$ \\
\hline 20 & $\begin{array}{l}\text { phenol, a very } \\
\text { important industrial } \\
\text { commodity as a } \\
\text { precursor to many } \\
\text { materials and useful } \\
\text { compounds }\end{array}$ & $\begin{array}{c}\text { CAS: } 108-95-2 \\
\text { AA Blocks } \\
(\$ 98 / 500 \mathrm{~g}, \$ 18 / \mathrm{mol}) \\
\text { Oakwood Chemicals } \\
(\$ 40 / 500 \mathrm{~g}, \\
\$ 7.3 / \mathrm{mmol})\end{array}$ & 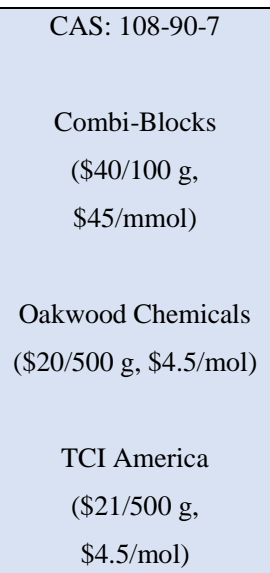 & $\begin{array}{c}\text { CAS: } 108-86-1 \\
\text { AA Blocks } \\
(\$ 40 / 100 \mathrm{~g}, \$ 63 / \mathrm{mol}) \\
\text { Combi-Blocks } \\
(\$ 250 / 1 \mathrm{~kg}, \$ 39 / \mathrm{mol}) \\
\text { Oakwood Chemicals } \\
(\$ 99 / 2.5 \mathrm{~kg}, \$ 6.2 / \mathrm{mol})\end{array}$ & $\begin{array}{c}\text { CAS: } 591-50-4 \\
\text { AA Blocks } \\
(\$ 150 / 500 \mathrm{~g}, \$ 61 / \mathrm{mol}) \\
\text { Ambeed, Inc. } \\
(\$ 98 / 500 \mathrm{~g}, \$ 40 / \mathrm{mol}) \\
\text { Combi-Blocks } \\
(\$ 250 / 1 \mathrm{~kg}, \$ 51 / \mathrm{mol})\end{array}$ \\
\hline 21 & $\begin{array}{l}\text { methyl salicylate, a } \\
\text { drug used to } \\
\text { temporarily } \\
\text { relieve minor body } \\
\text { aches and muscle and } \\
\text { joint pain. }\end{array}$ & $\begin{array}{c}\text { CAS: } 119-36-8 \\
\text { AA Blocks } \\
(\$ 57 / 500 \mathrm{~g}, \$ 17 / \mathrm{mol}) \\
\text { Ambeed, Inc. } \\
(\$ 30 / 500 \mathrm{~g}, \$ 8.9 / \mathrm{mol}) \\
\text { Combi-Blocks } \\
(\$ 250 / 1 \mathrm{~kg}, \$ 38 / \mathrm{mol})\end{array}$ & $\begin{array}{c}\text { CAS: 610-96-8 } \\
\text { AA Blocks } \\
(\$ 238 / 500 \mathrm{~g}, \$ 81 / \mathrm{mol}) \\
\text { Combi-Blocks } \\
(\$ 360 / 1 \mathrm{~kg}, \$ 61 / \mathrm{mol}) \\
\text { Oakwood Chemicals } \\
(\$ 50 / 100 \mathrm{~g}, \$ 85 / \mathrm{mol})\end{array}$ & $\begin{array}{c}\text { CAS: 610-94-6 } \\
\text { AA Blocks } \\
(\$ 187 / 500 \mathrm{~g}, \$ 80 / \mathrm{mol}) \\
\text { Ambeed, Inc. } \\
(\$ 197 / 1 \mathrm{~kg}, \$ 42 / \mathrm{mol}) \\
\text { Combi-Blocks } \\
(\$ 200 / 1 \mathrm{~kg}, \$ 43 / \mathrm{mol})\end{array}$ & $\begin{array}{c}\text { CAS: 610-97-9 } \\
\text { AA Blocks } \\
(\$ 19 / 100 \mathrm{~g}, \$ 50 / \mathrm{mol}) \\
\text { Ambeed, Inc. } \\
(\$ 99 / 500 \mathrm{~g}, \$ 52 / \mathrm{mol}) \\
\text { Combi-Blocks } \\
(\$ 150 / 500 \mathrm{~g}, \$ 79 / \mathrm{mol})\end{array}$ \\
\hline
\end{tabular}




\begin{tabular}{|c|c|c|c|c|c|}
\hline 22 & $\begin{array}{l}\text { sesamol, a natural } \\
\text { organic compound } \\
\text { which is a component } \\
\text { of sesame seeds and } \\
\text { sesame oil. }\end{array}$ & $\begin{array}{c}\text { CAS: } 533-31-3 \\
\text { AA Blocks } \\
(\$ 15 / 25 \mathrm{~g}, \$ 83 / \mathrm{mol}) \\
\text { Ambeed, Inc. } \\
(\$ 267 / 500 \mathrm{~g}, \$ 74 / \mathrm{mol}) \\
\text { Oakwood Chemicals } \\
(\$ 90 / 100 \mathrm{~g}, \$ 124 / \mathrm{mol})\end{array}$ & $\begin{array}{c}\text { CAS: } 7228-38-8 \\
\text { AA Blocks } \\
(\$ 25 / 5 \mathrm{~g}, \$ 780 / \mathrm{mol}) \\
\text { Matrix Scientific } \\
(\$ 89 / 5 \mathrm{~g}, \\
\$ 2,800 / \mathrm{mol}) \\
\text { Santa Cruz } \\
\text { Biotechnology } \\
(\$ 40 / 10 \mathrm{~g}, \\
\$ 620 / \mathrm{mol})\end{array}$ & $\begin{array}{c}\text { CAS: } 2635-13-4 \\
\text { AA Blocks } \\
(\$ 50 / 100 \mathrm{~g}, \$ 100 / \mathrm{mol}) \\
\text { Ambeed, Inc. } \\
(\$ 249 / 1000 \mathrm{~g}, \\
\$ 50 / \mathrm{mol}) \\
\text { Combi-Blocks } \\
(\$ 220 / 500 \mathrm{~g}, \$ 88 / \mathrm{mol})\end{array}$ & $\begin{array}{l}\text { CAS: 5876-51-7 } \\
\text { AA Blocks } \\
\left(\begin{array}{l}\$ 80 / 25 \mathrm{~g}, \$ 790 / \mathrm{mol}) \\
\\
\text { Ambeed, Inc. } \\
(\$ 344 / 100 \mathrm{~g}, \\
\$ 850 / \mathrm{mol}) \\
\text { Combi-Blocks } \\
(\$ 1,380 / 500 \mathrm{~g}, \\
\$ 680 / \mathrm{mol})\end{array}\right.\end{array}$ \\
\hline 23 & $\begin{array}{l}\text { 3'-hydroxy } \\
\text { acetophenone, a } \\
\text { component of } \\
\text { castoreum, the exudate } \\
\text { from the castor sacs of } \\
\text { the mature beaver. }\end{array}$ & $\begin{array}{c}\text { CAS: } 121-71-1 \\
\text { AA Blocks } \\
(\$ 34 / 100 \mathrm{~g}, \$ 46 / \mathrm{mol}) \\
\text { Ambeed, Inc. } \\
(\$ 98 / 500 \mathrm{~g}, \$ 27 / \mathrm{mol}) \\
\text { Combi-Blocks } \\
(\$ 250 / 1 \mathrm{~kg}, \$ 34 / \mathrm{mol})\end{array}$ & $\begin{array}{c}\text { CAS: } 99-02-5 \\
\text { AA Blocks } \\
(\$ 140 / 500 \mathrm{~g}, \$ 43 / \mathrm{mol}) \\
\text { Combi-Blocks } \\
(\$ 235 / 1 \mathrm{~kg}, \$ 36 / \mathrm{mol}) \\
\text { Oakwood Chemicals } \\
(\$ 270 / 1 \mathrm{~kg}, \$ 42 / \mathrm{mol})\end{array}$ & $\begin{array}{c}\text { CAS: } 2142-63-4 \\
\text { AA Blocks } \\
(\$ 181 / 500 \mathrm{~g}, \$ 72 / \mathrm{mol}) \\
\text { Ambeed, Inc. } \\
(\$ 256 / 1 \mathrm{~kg}, \$ 51 / \mathrm{mol}) \\
\text { Combi-Blocks } \\
(\$ 250 / 1 \mathrm{~kg}, \$ 50 / \mathrm{mol})\end{array}$ & $\begin{array}{l}\text { CAS: } 14452-30-3 \\
\text { AA Blocks } \\
(\$ 429 / 100 \mathrm{~g}, \\
\$ 610 / \mathrm{mol}) \\
\text { Ambeed, Inc. } \\
(\$ 95 / 25 \mathrm{~g}, \$ 930 / \mathrm{mol}) \\
\text { Combi-Blocks } \\
(\$ 240 / 25 \mathrm{~g}, \\
\$ 2400 / \mathrm{mol})\end{array}$ \\
\hline 24 & $\begin{array}{l}\text { methylparaben, an } \\
\text { anti-fungal agent often } \\
\text { used in a variety of } \\
\text { cosmetics and } \\
\text { personal-care products. }\end{array}$ & $\begin{array}{c}\text { CAS: } 99-76-3 \\
\text { AA Blocks } \\
(\$ 14 / 100 \mathrm{~g}, \$ 21 / \mathrm{mol}) \\
\text { Ambeed, Inc. } \\
(\$ 87 / 1 \mathrm{~kg}, \$ 13 / \mathrm{mol}) \\
\text { Oakwood Chemicals } \\
(\$ 390 / 2.5 \mathrm{~kg}, \\
\$ 24 / \mathrm{mol})\end{array}$ & $\begin{array}{c}\text { CAS: } 1126-46-1 \\
\text { AA Blocks } \\
(\$ 13 / 25 \mathrm{~g}, \$ 88 / \mathrm{mol}) \\
\text { Ambeed, Inc. } \\
\left(\begin{array}{l}\$ 241 / 1 \mathrm{~kg}, \$ 41 / \mathrm{mol}) \\
\text { Combi-Blocks } \\
(\$ 250 / 1 \mathrm{~kg}, \$ 43 / \mathrm{mol})\end{array}\right.\end{array}$ & $\begin{array}{c}\text { CAS: } 619-42-1 \\
\text { AA Blocks } \\
(\$ 150 / 500 \mathrm{~g}, \$ 65 / \mathrm{mol}) \\
\text { Ambeed, Inc. } \\
(\$ 245 / 1 \mathrm{~kg}, \$ 53 / \mathrm{mol}) \\
\text { Combi-Blocks } \\
(\$ 250 / 1 \mathrm{~kg}, \$ 54 / \mathrm{mol})\end{array}$ & $\begin{array}{c}\text { CAS: 619-44-3 } \\
\text { AA Blocks } \\
(\$ 58 / 100 \mathrm{~g}, \$ 150 / \mathrm{mol}) \\
\text { Ambeed, Inc. } \\
(\$ 274 / 500 \mathrm{~g}, \\
\$ 140 / \mathrm{mol}) \\
\text { Combi-Blocks } \\
(\$ 480 / 1 \mathrm{~kg}, \$ 130 / \mathrm{mol})\end{array}$ \\
\hline
\end{tabular}




\begin{tabular}{|c|c|c|c|c|c|}
\hline 25 & $\begin{array}{l}\text { precursor to } \\
\text { delamanid, a } \\
\text { medication used to } \\
\text { treat tuberculosis }\end{array}$ & $\begin{array}{c}\text { CAS: } 828-27-3 \\
\text { AA Blocks } \\
(\$ 193 / 500 \mathrm{~g}, \$ 69 / \mathrm{mol}) \\
\text { Ambeed, Inc. } \\
(\$ 247 / 1 \mathrm{~kg}, \$ 44 / \mathrm{mol}) \\
\text { Oakwood Chemicals } \\
(\$ 160 / 500 \mathrm{~g}, \$ 57 / \mathrm{mol})\end{array}$ & $\begin{array}{c}\text { CAS: } 461-81-4 \\
\text { AA Blocks } \\
(\$ 50 / 100 \mathrm{~g}, \$ 98 / \mathrm{mol}) \\
\text { Ambeed, Inc. } \\
(\$ 248 / 1 \mathrm{~kg}, \$ 49 / \mathrm{mol}) \\
\text { Combi-Blocks } \\
(\$ 280 / 500 \mathrm{~g}, \\
\$ 110 / \mathrm{mol})\end{array}$ & $\begin{array}{c}\text { CAS: } 407-14-7 \\
\text { AA Blocks } \\
(\$ 12 / 25 \mathrm{~g}, \$ 120 / \mathrm{mol}) \\
\text { Ambeed, Inc. } \\
(\$ 248 / 1 \mathrm{~kg}, \$ 60 / \mathrm{mol}) \\
\text { Combi-Blocks } \\
(\$ 320 / 1 \mathrm{~kg}, \$ 77 / \mathrm{mol})\end{array}$ & $\begin{array}{l}\text { CAS: } 103962-05-6 \\
\text { AA Blocks } \\
(\$ 145 / 100 \mathrm{~g}, \\
\$ 420 / \mathrm{mol}) \\
\text { Ambeed, Inc. } \\
(\$ 346 / 1 \mathrm{~kg}, \$ 99 / \mathrm{mol}) \\
\text { Combi-Blocks } \\
(\$ 350 / 1 \mathrm{~kg}, \$ 100 / \mathrm{mol})\end{array}$ \\
\hline 26 & $\begin{array}{l}\text { precursor to clofibrate, } \\
\text { a drug for controlling } \\
\text { the high cholesterol } \\
\text { and triacylglyceride } \\
\text { level in the blood }\end{array}$ & $\begin{array}{c}\text { CAS: } 106-48-9 \\
\text { AA Blocks } \\
(\$ 181 / 500 \mathrm{~g}, \$ 46 / \mathrm{mol}) \\
\\
\text { Oakwood Chemicals } \\
(\$ 14 / 25 \mathrm{~g}, \$ 72 / \mathrm{mol}) \\
\\
\text { Santa Cruz } \\
\text { Biotechnology } \\
(\$ 57 / 100 \mathrm{~g}, \\
\$ 73 / \mathrm{mol})\end{array}$ & $\begin{array}{c}\text { CAS: } 106-46-7 \\
\text { AA Blocks } \\
(\$ 60 / 100 \mathrm{~g}, \$ 88 / \mathrm{mol}) \\
\text { Combi-Blocks } \\
(\$ 250 / 1 \mathrm{~kg}, \$ 37 / \mathrm{mol}) \\
\text { Oakwood Chemicals } \\
(\$ 16 / 100 \mathrm{~g}, \$ 24 / \mathrm{mol})\end{array}$ & $\begin{array}{c}\text { CAS: } 106-39-8 \\
\text { AA Blocks } \\
(\$ 5 / 25 \mathrm{~g}, \$ 38 / \mathrm{mol}) \\
\text { Ambeed, Inc. } \\
(\$ 60 / 1 \mathrm{~kg}, \$ 11 / \mathrm{mol}) \\
\text { Combi-Blocks } \\
(\$ 250 / 1 \mathrm{~kg}, \$ 48 / \mathrm{mol})\end{array}$ & $\begin{array}{c}\text { CAS: 637-87-6 } \\
\text { AA Blocks } \\
(\$ 38 / 100 \mathrm{~g}, \$ 90 / \mathrm{mol}) \\
\text { Ambeed, Inc. } \\
(\$ 260 / 1 \mathrm{~kg}, \$ 62 / \mathrm{mol}) \\
\text { Combi-Blocks } \\
(\$ 250 / 1 \mathrm{~kg}, \$ 60 / \mathrm{mol})\end{array}$ \\
\hline 27 & $\begin{array}{l}\text { precursor to numerous } \\
\text { compounds (e.g., } \\
\text { fenitrothion, synthetic } \\
\text { vitamin E, and } \\
\text { amylmetacresol) }\end{array}$ & $\begin{array}{c}\text { CAS: } 108-39-4 \\
\text { AA Blocks } \\
(\$ 40 / 100 \mathrm{~g}, \$ 43 / \mathrm{mol}) \\
\text { Combi-Blocks } \\
(\$ 250 / 1 \mathrm{~kg}, \$ 27 / \mathrm{mol}) \\
\text { Oakwood Chemicals } \\
(\$ 62 / 1 \mathrm{~kg}, \$ 6.7 / \mathrm{mol})\end{array}$ & $\begin{array}{c}\text { CAS: } 108-41-8 \\
\text { AA Blocks } \\
(\$ 25 / 25 \mathrm{~g}, \$ 130 / \mathrm{mol}) \\
\text { Combi-Blocks } \\
(\$ 75 / 100 \mathrm{~g}, \$ 94 / \mathrm{mol}) \\
\text { Oakwood Chemicals } \\
(\$ 43 / 100 \mathrm{~g}, \$ 54 / \mathrm{mol})\end{array}$ & $\begin{array}{c}\text { CAS: } 591-17-3 \\
\text { AA Blocks } \\
(\$ 182 / 500 \mathrm{~g}, \$ 62 / \mathrm{mol}) \\
\text { Combi-Blocks } \\
(\$ 400 / 1 \mathrm{~kg}, \$ 68 / \mathrm{mol}) \\
\text { Oakwood Chemicals } \\
(\$ 186 / 500 \mathrm{~g}, \$ 64 / \mathrm{mol})\end{array}$ & $\begin{array}{c}\text { CAS: 625-95-6 } \\
\text { AA Blocks } \\
(\$ 200 / 500 \mathrm{~g}, \$ 87 / \mathrm{mol}) \\
\text { Ambeed, Inc. } \\
(\$ 372 / 1 \mathrm{~kg}, \$ 81 / \mathrm{mol}) \\
\text { Combi-Blocks } \\
(\$ 380 / 1 \mathrm{~kg}, \$ 83 / \mathrm{mol})\end{array}$ \\
\hline 28 & $\begin{array}{l}\text { precursor to } \\
\text { isoprocarb, an } \\
\text { agricultural insecticide } \\
\text { used on rice, cocoa, } \\
\text { sugar cane, vegetables } \\
\text { and other crops }\end{array}$ & $\begin{array}{c}\text { CAS: } 88-69-7 \\
\text { AA Blocks } \\
(\$ 70 / 100 \mathrm{~g}, \$ 95 / \mathrm{mol}) \\
\text { Combi-Blocks } \\
(\$ 50 / 100 \mathrm{~g}, \$ 68 / \mathrm{mol}) \\
\text { Santa Cruz } \\
\text { Biotechnology } \\
(\$ 185 / 500 \mathrm{~g}, \\
\$ 50 / \mathrm{mol})\end{array}$ & $\begin{array}{l}\text { CAS: 2077-13-6 } \\
\text { AA Blocks } \\
\text { (\$198/250 mg, } \\
\$ 121,000 / \mathrm{mol}) \\
\text { Combi-Blocks } \\
(\$ 450 / 1 \mathrm{~g}, \\
\$ 69,300 / \mathrm{mol})\end{array}$ & $\begin{array}{l}\text { CAS: 7073-94-1 } \\
\text { AA Blocks } \\
\left(\begin{array}{c}\$ 160 / 100 \mathrm{~g}, \\
\$ 320 / \mathrm{mol})\end{array}\right. \\
\text { Ambeed, Inc. } \\
\left(\begin{array}{l}\$ 52 / 500 \mathrm{~g}, \\
\$ 180 / \mathrm{mol})\end{array}\right. \\
\text { Combi-Blocks } \\
(\$ 620 / 500 \mathrm{~g}, \\
\$ 250 / \mathrm{mol})\end{array}$ & $\begin{array}{l}\text { CAS: 19099-54-8 } \\
\text { AA Blocks } \\
\left(\begin{array}{c}\$ 180 / 100 \mathrm{~g}, \\
\$ 440 / \mathrm{mol})\end{array}\right. \\
\text { Ambeed, Inc. } \\
\left(\begin{array}{l}\$ 977 / 1000 \mathrm{~g}, \\
\$ 240 / \mathrm{mol})\end{array}\right. \\
\text { Combi-Blocks } \\
(\$ 180 / 100 \mathrm{~g}, \\
\$ 440 / \mathrm{mol})\end{array}$ \\
\hline
\end{tabular}




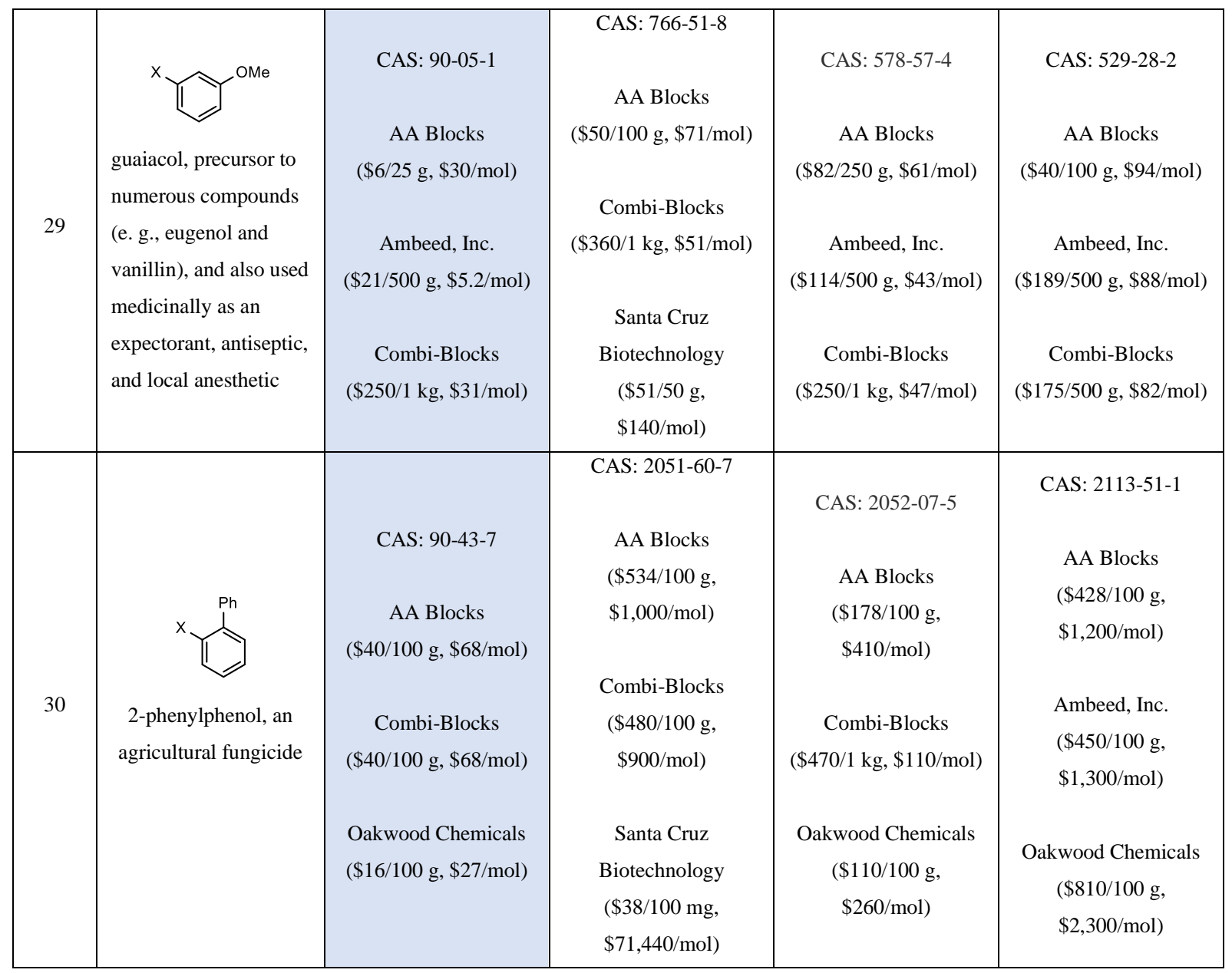




\section{Cross-Electrophile Couplings of Phenol Derivatives under Nickel and Palladium Multimetallic Conditions}

\subsection{Cross-Electrophile Couplings of Aryl Triflates with Aryl Tosylates}

Procedure for cross-electrophile couplings of aryl triflates with aryl tosylates on $\mathbf{0 . 5} \mathbf{~ m m o l ~ s c a l e ~}$

The following procedures were conducted in a $\mathrm{N}_{2}$ filled glovebox for convenience. Nickel and palladium catalyst stock solutions were prepared following general procedure for optimization studies (page S4) using $\mathbf{L 1}$ (18.5 mg, $0.060 \mathrm{mmol}$ ) as the amine ligand and dppb (25.6 $\mathrm{mg}, 0.060 \mathrm{mmol}$ ) as the phosphine ligand.

\section{General procedure A: catalytic reactions at 1.0 mol\% catalyst loading}

An oven-dried 1-dram vial equipped with a PTFE-coated stir-bar was charged sequentially with aryl triflate ( $0.50 \mathrm{mmol}, 1.0$ equiv), aryl tosylate $(0.50 \mathrm{mmol}, 1.0$ equiv or $0.75 \mathrm{mmol}, 1.5$ equiv $), \mathrm{LiBr}(174$ $\mathrm{mg}, 2.0 \mathrm{mmol}, 4.0$ equiv), and $\mathrm{Zn}(65 \mathrm{mg}, 1.0 \mathrm{mmol}, 2.0$ equiv). Aliquots from both the $0.025 \mathrm{M}$ nickel (200 $\mu \mathrm{L}$ for $1.0 \mathrm{~mol} \%$ ) and palladium ( $200 \mu \mathrm{L}$ for $1.0 \mathrm{~mol} \%$ ) catalyst stock solutions were added, followed by additional DMF (1.6 mL). The vial was capped with a screw cap fitted with a PTFE-faced silicone septum, and the reaction mixture was stirred $(1000 \mathrm{rpm})$ for $10 \mathrm{~min}$ in the glovebox at $\mathrm{rt}$. The vial was then removed from the glovebox and heated in a reaction block set to the desired temperature $\left(40\right.$ or $\left.60{ }^{\circ} \mathrm{C}\right)$ with stirring (1250 rpm) for $24 \mathrm{~h}$.

\section{General procedure B: catalytic reactions at $0.5 \mathrm{~mol} \%$ catalyst loading}

An oven-dried 1-dram vial equipped with a PTFE-coated stir-bar was charged sequentially with aryl triflate ( $0.50 \mathrm{mmol}, 1.0$ equiv), aryl tosylate $(0.50 \mathrm{mmol}, 1.0$ equiv or $0.75 \mathrm{mmol}, 1.5$ equiv $), \mathrm{LiBr}(174$ $\mathrm{mg}, 2.0 \mathrm{mmol}, 4.0$ equiv), and $\mathrm{Zn}(65 \mathrm{mg}, 1.0 \mathrm{mmol}, 2.0$ equiv). Aliquots from both the $0.025 \mathrm{M}$ nickel (100 $\mu \mathrm{L}$ for $0.5 \mathrm{~mol} \%$ ) and palladium ( $100 \mu \mathrm{L}$ for $0.5 \mathrm{~mol} \%$ ) catalyst stock solutions were added, followed by additional DMF $(0.8 \mathrm{~mL})$. The vial was capped with a screw cap fitted with a PTFE-faced silicone septum, and the reaction mixture was stirred $(1000 \mathrm{rpm})$ for $10 \mathrm{~min}$ in the glovebox at $\mathrm{rt}$. The vial was then removed from the glovebox and heated in a reaction block set to the desired temperature $\left(40\right.$ or $\left.60{ }^{\circ} \mathrm{C}\right)$ with stirring (1250 rpm) for $24 \mathrm{~h}$.

NOTE for Procedure B: we found that some aryl triflates slowly decomposed (a color change could be observed, usually from colorless to pale yellow) when they were stored in air at rt for several weeks. Reactions with these partially decomposed aryl triflates at $0.5 \mathrm{~mol} \%$ catalyst loadings usually provided low yields due to low conversion of starting materials. Aryl triflates used in general procedure B were used within 3 days of preparation. However, these partially decomposed aryl triflates could be repurified by flash chromatography on silica gel and used in reactions at $0.5 \mathrm{~mol} \%$ catalyst loading. 


\section{GC and SFC-MS analysis}

The reaction was monitored by GC analysis by cooling the reaction to rt and taking a $20 \mu \mathrm{L}$ aliquot of the crude reaction mixture with a gas-tight syringe. The aliquot was diluted with ethyl acetate $(1 \mathrm{~mL})$, filtered through a $2-\mathrm{cm}$ silica gel plug in a Pasteur pipette, and collected in a GC vial. The sample was analyzed by GC to determine the if the starting materials were consumed. SFC-MS analysis was used for reactions involving nonvolatile starting materials or products using the same workup as preparing GC samples.

\section{Isolation and purification}

Reaction completion was judged by GC or SFC-MS analysis and was usually accompanied by a color change from grey to dark brown. The reaction mixture was diluted with ethyl acetate $(10 \mathrm{~mL})$, filtered through a short plug of silica gel $(1.5 \mathrm{~cm} \times 3.0 \mathrm{~cm})$, and extra portions of ethyl acetate $(15 \mathrm{~mL} \times 5)$ were used to rinse the reaction vial and wash the silica gel pad. Silica gel (2.5-3.0 g) was then added to the combined filtrate and the volatile solvents were removed by rotary evaporation. The resulting dry-loaded material was purified by flash chromatography on silica gel to provide the desired product. 


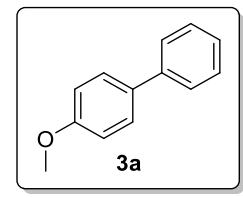

4-methoxy-1,1'-biphenyl (3a) [CAS: 613-37-6] was prepared following the general procedure A using 1a $(128 \mathrm{mg}, 0.50 \mathrm{mmol})$ and $\mathbf{2 a}(124 \mathrm{mg}, 0.50 \mathrm{mmol})$ as starting materials. After stirring at $40{ }^{\circ} \mathrm{C}$ for $24 \mathrm{~h}$, the reaction was quenched following general procedure and the crude material was purified by flash chromatography on silica gel (hexanes/ethyl acetate $=100 / 1$ to 50/1) to afford the desired product $(66.2$ mg, $72 \%$ ) as a white solid. ${ }^{1} \mathrm{H}$ NMR $\left(400 \mathrm{MHz}, \mathrm{CDCl}_{3}\right): \delta 7.56(\mathrm{t}, J=8.4 \mathrm{~Hz}, 4 \mathrm{H}), 7.43(\mathrm{t}, J=7.7 \mathrm{~Hz}, 2$ $\mathrm{H}), 7.31(\mathrm{t}, J=6.9 \mathrm{~Hz}, 1 \mathrm{H}), 6.99(\mathrm{~d}, J=8.7 \mathrm{~Hz}, 2 \mathrm{H}), 3.86(\mathrm{~s}, 3 \mathrm{H}) ;{ }^{13} \mathrm{C}\left\{{ }^{1} \mathrm{H}\right\} \mathrm{NMR}\left(100 \mathrm{MHZ}, \mathrm{CDCl}_{3}\right): \delta$ $159.3,141.0,134.0,128.9,128.3,126.9,126.8,114.4,55.5 \mathrm{ppm}$. Characterization data matched those reported in the literature. ${ }^{40}$

4-methoxy-1,1'-biphenyl (3a) [CAS: 613-37-6] was prepared following the general procedure B using 1a (128 mg, $0.50 \mathrm{mmol})$ and $\mathbf{2 a}(124 \mathrm{mg}, 0.50 \mathrm{mmol})$ as starting materials. After stirring at $40{ }^{\circ} \mathrm{C}$ for $24 \mathrm{~h}$, the reaction was quenched following general procedure and the crude material was purified by flash chromatography on silica gel (hexanes/ethyl acetate $=100 / 1$ to $50 / 1$ ) to afford the desired product (65.3 mg, 71\%) as a white solid. ${ }^{1} \mathrm{H}$ NMR (400 MHz, $\left.\mathrm{CDCl}_{3}\right) \delta 7.57(\mathrm{t}, J=8.5 \mathrm{~Hz}, 4 \mathrm{H}), 7.44$ (t, $J=7.7 \mathrm{~Hz}, 2$ $\mathrm{H}), 7.33(\mathrm{t}, J=7.3 \mathrm{~Hz}, 1 \mathrm{H}), 7.01(\mathrm{~d}, J=8.8 \mathrm{~Hz}, 2 \mathrm{H}), 3.87(\mathrm{~s}, 3 \mathrm{H}) ;{ }^{13} \mathrm{C}\left\{{ }^{1} \mathrm{H}\right\} \mathrm{NMR}\left(100 \mathrm{MHz}, \mathrm{CDCl}_{3}\right) \delta$ $159.3,141.0,133.9,128.9,128.3,126.9,126.8,114.4,55.5$ ppm. Characterization data matched those reported in the literature. ${ }^{40}$

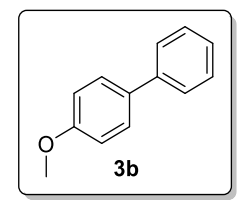

4-methoxy-1,1'-biphenyl (3b) [CAS: 613-37-6] was prepared following the general procedure A using 1b (113 mg, $0.50 \mathrm{mmol})$ and $\mathbf{2 b}(139 \mathrm{mg}, 0.50 \mathrm{mmol})$ as starting materials. After stirring at $40{ }^{\circ} \mathrm{C}$ for $24 \mathrm{~h}$, the reaction was quenched following general procedure and the crude material was purified by flash chromatography on silica gel (hexanes/ethyl acetate $=100 / 1$ to $50 / 1)$ to afford the desired product $(57.0$ mg, 62\%) as a white solid. ${ }^{1} \mathrm{H}$ NMR (400 MHz, $\left.\mathrm{CDCl}_{3}\right) \delta 7.56(\mathrm{t}, J=8.3 \mathrm{~Hz}, 4 \mathrm{H}), 7.44$ (t, $J=7.6 \mathrm{~Hz}, 2$ $\mathrm{H}), 7.32(\mathrm{t}, J=7.4 \mathrm{~Hz}, 1 \mathrm{H}), 7.00(\mathrm{~d}, J=8.7 \mathrm{~Hz}, 2 \mathrm{H}), 3.87(\mathrm{~s}, 3 \mathrm{H}) ;{ }^{13} \mathrm{C}\left\{{ }^{1} \mathrm{H}\right\} \mathrm{NMR}\left(100 \mathrm{MHz}, \mathrm{CDCl}_{3}\right) \delta$ $159.3,141.0,133.9,128.9,128.3,126.9,126.8,114.4,55.5$ ppm. Characterization data matched those reported in the literature. ${ }^{40}$ 


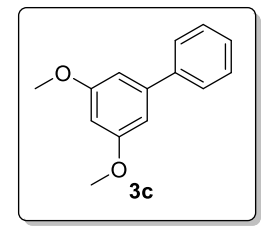

3,5-dimethoxy-1,1'-biphenyl (3c) [CAS: 64326-17-6] was prepared following the general procedure A using 1c (143 mg, $0.50 \mathrm{mmol})$ and $\mathbf{2 a}(124 \mathrm{mg}, 0.50 \mathrm{mmol})$ as starting materials. After stirring at $40{ }^{\circ} \mathrm{C}$ for $24 \mathrm{~h}$, the reaction was quenched following general procedure and the crude material was purified by flash chromatography on silica gel (hexanes/ethyl acetate $=50 / 1$ to $30 / 1$ ) to afford the desired product (75.5 mg, $70 \%$ ) as a white solid. ${ }^{1} \mathrm{H}$ NMR $\left(500 \mathrm{MHz}, \mathrm{CDCl}_{3}\right): \delta 7.62(\mathrm{dd}, J=8.2,1.3 \mathrm{~Hz}, 2 \mathrm{H}), 7.47$ (t, $J=7.6 \mathrm{~Hz}, 2$ $\mathrm{H}), 7.39(\mathrm{t}, J=7.3 \mathrm{~Hz}, 1 \mathrm{H}), 6.79(\mathrm{~d}, J=2.3 \mathrm{~Hz}, 2 \mathrm{H}), 6.52(\mathrm{t}, J=2.3 \mathrm{~Hz}, 1 \mathrm{H}), 3.88(\mathrm{~s}, 6 \mathrm{H}) ;{ }^{13} \mathrm{C}\left\{{ }^{1} \mathrm{H}\right\}$ NMR (125 MHz, $\left.\mathrm{CDCl}_{3}\right): \delta 161.2,143.6,141.4,128.8,127.7,127.3,105.6,99.4,55.5$ ppm. Characterization data matched those reported in the literature. ${ }^{41}$

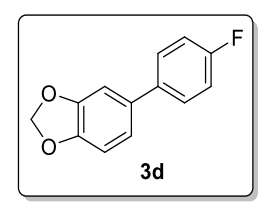

5-(4-fluorophenyl)benzo[d][1,3]dioxole (3d) [CAS: 157790-85-7] was prepared following the general procedure A using 1d (135 mg, $0.50 \mathrm{mmol})$ and $2 \mathbf{c}(133 \mathrm{mg}, 0.50 \mathrm{mmol})$ as starting materials. After stirring at $40{ }^{\circ} \mathrm{C}$ for $24 \mathrm{~h}$, the reaction was quenched following general procedure and the crude material was purified by flash chromatography on silica gel (hexanes/ethyl acetate $=100 / 1$ to 50/1) to afford the desired product $(79.2 \mathrm{mg}, 73 \%)$ as a white solid. ${ }^{1} \mathrm{H} \mathrm{NMR}\left(400 \mathrm{MHz}, \mathrm{CDCl}_{3}\right): \delta 7.48-7.45(\mathrm{~m}, 2 \mathrm{H}), 7.10(\mathrm{t}, J=$ $8.7 \mathrm{~Hz}, 2 \mathrm{H}), 7.03-6.99(\mathrm{~m}, 2 \mathrm{H}), 6.88(\mathrm{~d}, J=8.0 \mathrm{~Hz}, 1 \mathrm{H}), 6.00(\mathrm{~s}, 2 \mathrm{H}) ;{ }^{13} \mathrm{C}\left\{{ }^{1} \mathrm{H}\right\} \mathrm{NMR}(100 \mathrm{MHz}$, $\left.\mathrm{CDCl}_{3}\right): \delta 162.5(\mathrm{~d}, J=247.5 \mathrm{~Hz}), 148.3,147.2,137.2(\mathrm{~d}, J=3.0 \mathrm{~Hz}), 134.8,128.5(\mathrm{~d}, J=8.1 \mathrm{~Hz}), 120.6$, $115.7(\mathrm{~d}, J=21.2 \mathrm{~Hz}), 108.8,107.7,101.4 ;{ }^{19} \mathrm{~F}\left\{{ }^{1} \mathrm{H}\right\}$ NMR (376 $\left.\mathrm{MHz}, \mathrm{CDCl}_{3}\right): \delta-116.20 \mathrm{ppm}$. Characterization data matched those reported in the literature. ${ }^{42}$

5-(4-fluorophenyl)benzo[d][1,3]dioxole (3d) [CAS: 157790-85-7] was prepared following the general procedure B using 1d $(135 \mathrm{mg}, 0.50 \mathrm{mmol})$ and $2 \mathrm{c}(133 \mathrm{mg}, 0.50 \mathrm{mmol})$ as starting materials. After stirring at $40{ }^{\circ} \mathrm{C}$ for $24 \mathrm{~h}$, the reaction was quenched following general procedure and the crude material was purified by flash chromatography on silica gel (hexanes/ethyl acetate $=100 / 1$ to 50/1) to afford the desired product $(75.3 \mathrm{mg}, 70 \%)$ as a white solid. ${ }^{1} \mathrm{H} \mathrm{NMR}\left(400 \mathrm{MHz}, \mathrm{CDCl}_{3}\right) \delta 7.48-7.45(\mathrm{~m}, 2 \mathrm{H}), 7.10(\mathrm{t}, J=$ $8.7 \mathrm{~Hz}, 2 \mathrm{H}), 7.02-6.99(\mathrm{~m}, 2 \mathrm{H}), 6.88(\mathrm{~d}, J=7.9 \mathrm{~Hz}, 1 \mathrm{H}), 6.00(\mathrm{~s}, 2 \mathrm{H}) ;{ }^{13} \mathrm{C}\left\{{ }^{1} \mathrm{H}\right\} \mathrm{NMR}(100 \mathrm{MHz}$, $\left.\mathrm{CDCl}_{3}\right): \delta 162.4(\mathrm{~d}, J=245.9 \mathrm{~Hz}), 148.3,147.2,137.2(\mathrm{~d}, J=3.2 \mathrm{~Hz}), 134.8,128.6(\mathrm{~d}, J=7.9 \mathrm{~Hz}), 120.7$, 
$115.7(\mathrm{~d}, J=21.4 \mathrm{~Hz}), 108.8,107.8,101.4 ;{ }^{19} \mathrm{~F}\left\{{ }^{1} \mathrm{H}\right\}$ NMR $\left(376 \mathrm{MHz}, \mathrm{CDCl}_{3}\right): \delta-116.22 \mathrm{ppm}$. Characterization data matched those reported in the literature. ${ }^{42}$

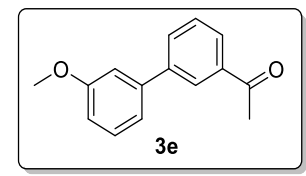

1-(3'-methoxy-[1,1'-biphenyl]-3-yl)ethan-1-one (3e) [CAS: 843734-63-9] was prepared following the general procedure A using 1 e $(128 \mathrm{mg}, 0.50 \mathrm{mmol})$ and $\mathbf{2 d}(145 \mathrm{mg}, 0.50 \mathrm{mmol})$ as starting materials. After stirring at $40{ }^{\circ} \mathrm{C}$ for $24 \mathrm{~h}$, the reaction was quenched following general procedure and the crude material was purified by flash chromatography on silica gel (hexanes/ethyl acetate $=20 / 1$ to $10 / 1$ ) to afford the desired product (64.4 mg, 57\%) as a white solid. ${ }^{1} \mathrm{H} \mathrm{NMR}\left(400 \mathrm{MHz}, \mathrm{CDCl}_{3}\right): \delta 8.18(\mathrm{t}, J=1.8 \mathrm{~Hz}, 1 \mathrm{H})$, $7.94(\mathrm{~d}, J=7.6 \mathrm{~Hz}, 1 \mathrm{H}), 7.78(\mathrm{~d}, J=7.7 \mathrm{~Hz}, 1 \mathrm{H}), 7.53(\mathrm{t}, J=7.7 \mathrm{~Hz}, 1 \mathrm{H}), 7.39$ (t, $J=8.2 \mathrm{~Hz}, 1 \mathrm{H}), 7.20$ $(\mathrm{d}, J=7.5 \mathrm{~Hz}, 1 \mathrm{H}), 7.14(\mathrm{t}, J=2.1 \mathrm{~Hz}, 1 \mathrm{H}), 6.94(\mathrm{dd}, J=7.8,3.1 \mathrm{~Hz}, 1 \mathrm{H}), 3.88(\mathrm{~s}, 3 \mathrm{H}), 2.66(\mathrm{~s}, 3 \mathrm{H})$; ${ }^{13} \mathrm{C}\left\{{ }^{1} \mathrm{H}\right\}$ NMR $\left(125 \mathrm{MHz}, \mathrm{CDCl}_{3}\right): \delta 198.2,160.2,141.9,141.8,137.8,132.0,130.1,129.2,127.5,127.2$, $119.8,113.3,113.2,55.5,27.0 \mathrm{ppm}$. Characterization data matched those reported in the literature. ${ }^{43}$

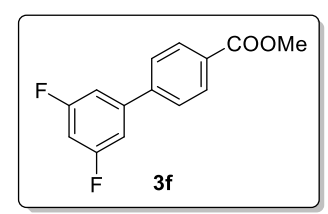

Methyl 3',5'-difluoro-[1,1'-biphenyl]-4-carboxylate (3f) [CAS: 1848978-85-7] was prepared following the general procedure A using $\mathbf{1 f}(131 \mathrm{mg}, 0.50 \mathrm{mmol})$ and $\mathbf{2 e}(153 \mathrm{mg}, 0.50 \mathrm{mmol})$ as starting materials. After stirring at $40{ }^{\circ} \mathrm{C}$ for $24 \mathrm{~h}$, the reaction was quenched following general procedure and the crude material was purified by flash chromatography on silica gel (hexanes/ethyl acetate $=20 / 1$ to 5/1) to afford the desired product $(63.1 \mathrm{mg}, 51 \%)$ as a white solid. ${ }^{1} \mathrm{H} \mathrm{NMR}\left(500 \mathrm{MHz}, \mathrm{CDCl}_{3}\right): \delta 8.11(\mathrm{~d}, J=8.5 \mathrm{~Hz}, 2$ $\mathrm{H}), 7.60(\mathrm{~d}, J=8.4 \mathrm{~Hz}, 2 \mathrm{H}), 7.12(\mathrm{~m}, 2 \mathrm{H}), 6.82(\mathrm{tt}, J=8.8,2.3 \mathrm{~Hz}, 1 \mathrm{H}), 3.94(\mathrm{~s}, 3 \mathrm{H}) ;{ }^{13} \mathrm{C}\left\{{ }^{1} \mathrm{H}\right\} \mathrm{NMR}$ $\left(125 \mathrm{MHz}, \mathrm{CDCl}_{3}\right): \delta 166.8,163.5(\mathrm{dd}, J=249.5,13.9 \mathrm{~Hz}), 143.4(\mathrm{t}, J=9.4 \mathrm{~Hz}), 143.2(\mathrm{t}, J=2.5 \mathrm{~Hz})$, $130.4,130.2,127.1,110.3(\mathrm{~m}), 103.5(\mathrm{t}, J=25.2 \mathrm{~Hz}), 52.4 ;{ }^{19} \mathrm{~F}\left\{{ }^{1} \mathrm{H}\right\} \mathrm{NMR}\left(376 \mathrm{MHz}, \mathrm{CDCl}_{3}\right): \delta-109.14$ (s, 2 F) ppm. Characterization data matched those reported in the literature. ${ }^{44}$

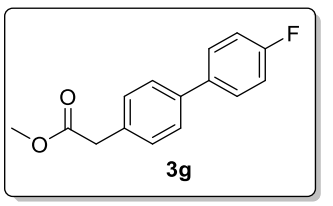


Methyl 2-(4'-fluoro-[1,1'-biphenyl]-4-yl)acetate (3g) [CAS: 1949812-56-9] was prepared following the general procedure A using $1 \mathrm{~g}(149 \mathrm{mg}, 0.50 \mathrm{mmol})$ and $\mathbf{2 c}(133 \mathrm{mg}, 0.50 \mathrm{mmol})$ as starting materials. After stirring at $40{ }^{\circ} \mathrm{C}$ for $24 \mathrm{~h}$, the reaction was quenched following general procedure and the crude material was purified by flash chromatography on silica gel (hexanes/ethyl acetate $=20 / 1$ to $5 / 1$ ) to afford the desired product $(86.8 \mathrm{mg}, 71 \%)$ as a colorless oil. ${ }^{1} \mathrm{H}$ NMR $\left(400 \mathrm{MHz}, \mathrm{CDCl}_{3}\right): \delta 7.55-7.50(\mathrm{~m}, 4 \mathrm{H}), 7.35(\mathrm{~d}, J$ $=8.3 \mathrm{~Hz}, 2 \mathrm{H}), 7.12(\mathrm{t}, J=8.7 \mathrm{~Hz}, 2 \mathrm{H}), 3.72(\mathrm{~s}, 3 \mathrm{H}), 3.68(\mathrm{~s}, 2 \mathrm{H}) ;{ }^{13} \mathrm{C}\left\{{ }^{1} \mathrm{H}\right\} \mathrm{NMR}\left(125 \mathrm{MHz}, \mathrm{CDCl}_{3}\right): \delta$ 172.2, $162.6(\mathrm{~d}, J=252.0 \mathrm{~Hz}), 139.3,137.1$ (d, $J=3.8 \mathrm{~Hz}), 133.2,129.9,128.8$ (d, $J=7.6 \mathrm{~Hz}), 127.4$, $115.8(\mathrm{~d}, J=21.4 \mathrm{~Hz}), 52.3,40.9 ;{ }^{19} \mathrm{~F}\left\{{ }^{1} \mathrm{H}\right\}$ NMR (376 MHz, $\left.\mathrm{CDCl}_{3}\right): \delta-115.74 \mathrm{ppm}$. HRMS (ESI) m/z calcd for $[\mathrm{M}+\mathrm{Na}]^{+}$267.0792, found: 267.0787. IR $\left(\mathrm{cm}^{-1}\right)$ : 3045, 2952, 1738, 1499, 1231, 1160, 807.

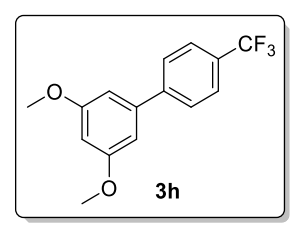

3,5-dimethoxy-4' -(trifluoromethyl)-1,1'-biphenyl (3h) [CAS: 216679-41-3] was prepared following the general procedure A using $\mathbf{1 c}(143 \mathrm{mg}, 0.50 \mathrm{mmol})$ and $\mathbf{2 f}(158 \mathrm{mg}, 0.50 \mathrm{mmol})$ as starting materials. After stirring at $40{ }^{\circ} \mathrm{C}$ for $24 \mathrm{~h}$, the reaction was quenched following general procedure and the crude material was purified by flash chromatography on silica gel (hexanes/ethyl acetate $=50 / 1$ to 10/1) to afford the desired product $(85.3 \mathrm{mg}, 60 \%)$ as a white solid. ${ }^{1} \mathrm{H}$ NMR $\left(500 \mathrm{MHz}, \mathrm{CDCl}_{3}\right): \delta 7.68(\mathrm{~s}, 4 \mathrm{H}), 6.72(\mathrm{~d}, J=$ $5.0 \mathrm{~Hz}, 2 \mathrm{H}), 6.52(\mathrm{t}, J=5.0 \mathrm{~Hz}, 1 \mathrm{H}), 3.86(\mathrm{~s}, 6 \mathrm{H}) ;{ }^{13} \mathrm{C}\left\{{ }^{1} \mathrm{H}\right\} \mathrm{NMR}\left(125 \mathrm{MHz}, \mathrm{CDCl}_{3}\right): \delta$ 161.4, 144.9, 142.1, 129.8 (q, $J=32.3 \mathrm{~Hz}), 127.7,125.8$ (q, $J=4.2 \mathrm{~Hz}), 124.4$ (q, $J=273.4 \mathrm{~Hz}), 105.8,100.1,55.6$; ${ }^{19} \mathrm{~F}\left\{{ }^{1} \mathrm{H}\right\}$ NMR (376 MHz, $\mathrm{CDCl}_{3}$ ): $\delta-62.42$ (s, $3 \mathrm{~F}$ ) ppm. Characterization data matched those reported in the literature. ${ }^{44}$

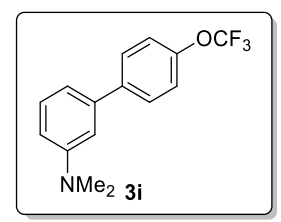

$N, N$-dimethyl-4' -(trifluoromethoxy)-[1,1'-biphenyl]-3-amine (3i) [CAS: 1261447-26-0] was prepared following the general procedure A using $1 \mathbf{h}(135 \mathrm{mg}, 0.50 \mathrm{mmol})$ and $\mathbf{2 g}(166 \mathrm{mg}, 0.50 \mathrm{mmol})$ as starting materials. After stirring at $40{ }^{\circ} \mathrm{C}$ for $24 \mathrm{~h}$, the reaction was quenched following general procedure and the crude material was purified by flash chromatography on silica gel (hexanes/ethyl acetate $=50 / 1$ to $20 / 1$ ) to afford the desired product $(94.3 \mathrm{mg}, 67 \%)$ as a colorless oil. Compound 3 i turned yellow immediately when it was dissolved in $\mathrm{CDCl}_{3}$ and the NMR data were obtained in $C_{6} D_{6} .{ }^{1} \mathrm{H} \mathrm{NMR}\left(500 \mathrm{MHz}, \mathrm{C}_{6} \mathrm{D}_{6}\right): \delta 7.33-$ 
$7.30(\mathrm{~m}, 2 \mathrm{H}), 7.24$ (t, $J=8.0 \mathrm{~Hz}, 1 \mathrm{H}), 7.00(\mathrm{~d}, J=7.3 \mathrm{~Hz}, 2 \mathrm{H}), 6.86(\mathrm{~d}, J=7.7 \mathrm{~Hz}, 1 \mathrm{H}), 6.76(\mathrm{t}, J=2.1$ $\mathrm{Hz}, 1 \mathrm{H}), 6.59(\mathrm{dd}, J=7.5,2.7 \mathrm{~Hz}, 1 \mathrm{H}), 2.54(\mathrm{~s}, 6 \mathrm{H}) ;{ }^{13} \mathrm{C}\left\{{ }^{1} \mathrm{H}\right\} \mathrm{NMR}\left(125 \mathrm{MHz}, \mathrm{C}_{6} \mathrm{D}_{6}\right): \delta 151.3,148.8$, $141.8,141.2,129.8,129.0,121.4,121.3(\mathrm{q}, J=257.0 \mathrm{~Hz}), 116.1,112.4,111.7,40.2 ;{ }^{19} \mathrm{~F}\left\{{ }^{1} \mathrm{H}\right\} \mathrm{NMR}(376$ $\left.\mathrm{MHz}, \mathrm{C}_{6} \mathrm{D}_{6}\right): \delta-57.61\left(\mathrm{~s}, 3 \mathrm{~F}\right.$ ) ppm. HRMS (ESI) $\mathrm{m} / \mathrm{z}$ calcd for $[\mathrm{M}+\mathrm{H}]^{+} 282.1100$, found 282.1099. IR $\left(\mathrm{cm}^{-1}\right): 3041,2805,1682,1493,1154,837,772$.

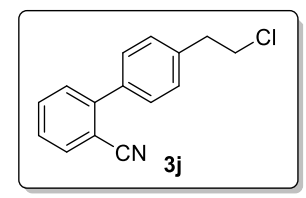

4'-(2-chloroethyl)-[1,1'-biphenyl]-2-carbonitrile (3j) was prepared following the general procedure A using $1 \mathbf{i}(144 \mathrm{mg}, 0.50 \mathrm{mmol})$ and $\mathbf{2 h}(137 \mathrm{mg}, 0.50 \mathrm{mmol})$ as starting materials. After stirring at $40{ }^{\circ} \mathrm{C}$ for $24 \mathrm{~h}$, the reaction was quenched following general procedure and the crude material was purified by flash chromatography on silica gel (hexanes/ethyl acetate $=20 / 1$ to $5 / 1)$ to afford the desired product $(62.3 \mathrm{mg}$, $51 \%)$ as a colorless oil. ${ }^{1} \mathrm{H} \mathrm{NMR}\left(500 \mathrm{MHz}, \mathrm{CDCl}_{3}\right): \delta 7.76(\mathrm{~d}, J=7.2 \mathrm{~Hz}, 1 \mathrm{H}), 7.63(\mathrm{t}, J=7.7 \mathrm{~Hz}, 1 \mathrm{H})$, $7.52(\mathrm{t}, J=8.0 \mathrm{~Hz}, 3 \mathrm{H}), 7.44(\mathrm{t}, J=7.6 \mathrm{~Hz}, 1 \mathrm{H}), 7.35(\mathrm{~d}, J=8.0 \mathrm{~Hz}, 2 \mathrm{H}), 3.77$ (t, $J=7.4 \mathrm{~Hz}, 2 \mathrm{H}), 3.14$ $(\mathrm{t}, J=7.4 \mathrm{~Hz}, 2 \mathrm{H}) ;{ }^{13} \mathrm{C}\left\{{ }^{1} \mathrm{H}\right\} \mathrm{NMR}\left(125 \mathrm{MHz}, \mathrm{CDCl}_{3}\right): \delta 145.3,138.9,136.9,133.9,133.0,130.2,129.4$, 129.1, 127.7, 118.9, 111.3, 44.8, $39.0 \mathrm{ppm}$. HRMS (ESI) $\mathrm{m} / \mathrm{z}$ calcd for $\left[\mathrm{M}+\mathrm{NH}_{4}\right]^{+} 259.0997$, found 259.0993. IR $\left(\mathrm{cm}^{-1}\right): 3028,2959,2223,1478,1266,762,735$.

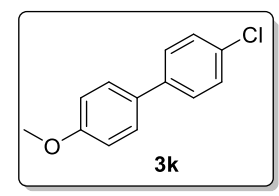

4-chloro-4'-methoxy-1,1'-biphenyl (3k) [CAS: 58970-19-7] was prepared following the general procedure A using 1a $(128 \mathrm{mg}, 0.50 \mathrm{mmol})$ and $2 \mathbf{i}(141 \mathrm{mg}, 0.50 \mathrm{mmol})$ as starting materials. After stirring at $35{ }^{\circ} \mathrm{C}$ for $24 \mathrm{~h}$, the reaction was quenched following general procedure and the crude material was purified by flash chromatography on silica gel (hexanes/ethyl acetate $=100 / 1$ to 50/1) to afford the desired product (61.8 mg, 57\%) as a white solid. ${ }^{1} \mathrm{H} \mathrm{NMR}\left(500 \mathrm{MHz}, \mathrm{CDCl}_{3}\right): \delta 7.49$ (t, $\left.J=8.3 \mathrm{~Hz}, 4 \mathrm{H}\right), 7.38(\mathrm{~d}$, $J=8.5 \mathrm{~Hz}, 2 \mathrm{H}), 6.98(\mathrm{~d}, J=8.7 \mathrm{~Hz}, 2 \mathrm{H}), 3.86(\mathrm{~s}, 3 \mathrm{H}) ;{ }^{13} \mathrm{C}\left\{{ }^{1} \mathrm{H}\right\} \mathrm{NMR}\left(125 \mathrm{MHz}, \mathrm{CDCl}_{3}\right): \delta 159.5,139.4$, $132.8,132.6,129.0,128.2,128.1,114.5,55.5 \mathrm{ppm}$. Characterization data matched those reported in the literature. $^{45}$ 


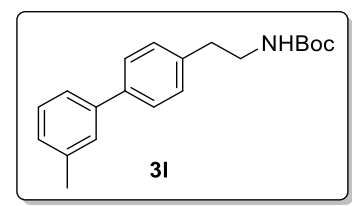

tert-butyl (2-(3'-methyl-[1,1'-biphenyl]-4-yl)ethyl)carbamate (3l) was prepared following the general procedure A using $\mathbf{1 j}$ (135 $\mathrm{mg}, 0.50 \mathrm{mmol})$ and $\mathbf{2} \mathbf{j}$ (131 $\mathrm{mg}, 0.50 \mathrm{mmol}$ ) as starting materials. After stirring at $40{ }^{\circ} \mathrm{C}$ for $24 \mathrm{~h}$, the reaction was quenched following general procedure and the crude material was purified by flash chromatography on silica gel (hexanes/ethyl acetate $=50 / 1$ to 20/1) to afford the desired product $(116.3 \mathrm{mg}, 75 \%)$ as a white solid. $\mathrm{mp}=88-90{ }^{\circ} \mathrm{C} .{ }^{1} \mathrm{H} \mathrm{NMR}\left(500 \mathrm{MHz}, \mathrm{CDCl}_{3}\right): \delta 7.55(\mathrm{~d}, J=8.2$ $\mathrm{Hz}, 2 \mathrm{H}), 7.38$ (d, $J=11.8 \mathrm{~Hz}, 2 \mathrm{H}), 7.34(\mathrm{t}, J=7.5 \mathrm{~Hz}, 1 \mathrm{H}), 7.28$ (d, $J=7.8 \mathrm{~Hz}, 2 \mathrm{H}), 7.18$ (d, $J=7.4 \mathrm{~Hz}$, $2 \mathrm{H}), 4.64(\mathrm{~s}, 1 \mathrm{H}), 3.44(\mathrm{q}, J=5.0 \mathrm{~Hz}, 2 \mathrm{H}), 2.85(\mathrm{t}, J=7.1 \mathrm{~Hz}, 2 \mathrm{H}), 2.44(\mathrm{~s}, 3 \mathrm{H}), 1.47(\mathrm{~s}, 9 \mathrm{H}) ;{ }^{13} \mathrm{C}\left\{{ }^{1} \mathrm{H}\right\}$ NMR (125 MHz, $\left.\mathrm{CDCl}_{3}\right): \delta 156.0,141.0,139.6,138.4,138.1,129.3,128.8,128.0,127.9,127.4,124.2$, 79.3, 41.9, 36.0, 28.6, $21.7 \mathrm{ppm}$. HRMS (ESI) $\mathrm{m} / \mathrm{z}$ calcd for $[\mathrm{M}+\mathrm{Na}]^{+} 334.1778$, found 334.1773. IR $\left(\mathrm{cm}^{-1}\right): 3365,2982,1682,1525,1246,1165,777$.

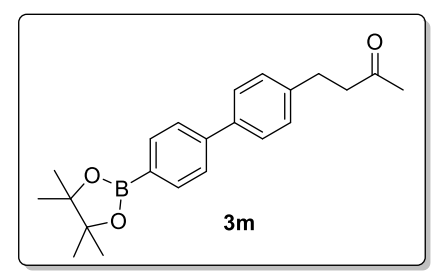

4-(4'-(4,4,5,5-tetramethyl-1,3,2-dioxaborolan-2-yl)-[1,1'-biphenyl]-4-yl)butan-2-one $\quad(3 \mathrm{~m}) \quad$ was prepared following the general procedure A using $1 \mathbf{k}(148 \mathrm{mg}, 0.50 \mathrm{mmol})$ and $\mathbf{2 k}(280 \mathrm{mg}, 0.75 \mathrm{mmol})$ as starting materials. After stirring at $40{ }^{\circ} \mathrm{C}$ for $24 \mathrm{~h}$, the reaction was quenched following general procedure and the crude material was purified by flash chromatography on silica gel (hexanes/ethyl acetate $=10 / 1$ to $5 / 1)$ to afford the desired product $(118.5 \mathrm{mg}, 68 \%)$ as a white solid. $\mathrm{mp}=127-129{ }^{\circ} \mathrm{C} .{ }^{1} \mathrm{H}$ NMR $(500$ $\left.\mathrm{MHz}_{\mathrm{CDCl}}\right): \delta 7.88(\mathrm{~d}, J=8.1 \mathrm{~Hz}, 2 \mathrm{H}), 7.69(\mathrm{~d}, J=8.1 \mathrm{~Hz}, 2 \mathrm{H}), 7.55(\mathrm{~d}, J=8.1 \mathrm{~Hz}, 2 \mathrm{H}), 7.26(\mathrm{~d}, J=$ $8.0 \mathrm{~Hz}, 2 \mathrm{H}), 2.95(\mathrm{t}, J=7.6 \mathrm{~Hz}, 2 \mathrm{H}), 2.80(\mathrm{t}, J=7.7 \mathrm{~Hz}, 2 \mathrm{H}), 2.16(\mathrm{~s}, 3 \mathrm{H}), 1.36(\mathrm{~s}, 12 \mathrm{H}) ;{ }^{13} \mathrm{C}\left\{{ }^{1} \mathrm{H}\right\} \mathrm{NMR}$ $\left(125 \mathrm{MHz}, \mathrm{CDCl}_{3}\right): \delta 208.0,143.7,140.7,139.0,135.4,128.9,127.5,126.4,84.0,45.3,30.3,29.5,25.0$ ppm. HRMS (ESI) $\mathrm{m} / \mathrm{z}$ calcd for $\left[\mathrm{M}+\mathrm{NH}_{4}\right]^{+}$368.2392, found 368.2393. IR $\left(\mathrm{cm}^{-1}\right): 2983,2931,1713,1605$, $1354,1320,1139,853,812,656$. The ${ }^{13} \mathrm{C}$ NMR spectrum is missing one signal, which is the carbon directly bound to boron. Due to coupling with both quadrupolar boron isotopes, this carbon is challenging to resolve. $^{46}$ 


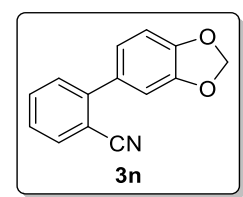

2-(benzo[d][1,3]dioxol-5-yl)benzonitrile (3n) [CAS: 475278-18-3] was prepared following the general procedure A using $\mathbf{1 d}(135 \mathrm{mg}, 0.50 \mathrm{mmol})$ and $\mathbf{2 h}(137 \mathrm{mg}, 0.50 \mathrm{mmol})$ as starting materials. After stirring at $40{ }^{\circ} \mathrm{C}$ for $24 \mathrm{~h}$, the reaction was quenched following general procedure and the crude material was purified by flash chromatography on silica gel (hexanes/ethyl acetate $=20 / 1$ to $5 / 1$ ) to afford the desired product $(80.2 \mathrm{mg}, 72 \%)$ as a white solid. ${ }^{1} \mathrm{H} \mathrm{NMR}\left(500 \mathrm{MHz}, \mathrm{CDCl}_{3}\right): 7.73(\mathrm{dd}, J=7.7,1.4 \mathrm{~Hz}, 1 \mathrm{H}), 7.61$ $(\mathrm{td}, J=7.7,1.4 \mathrm{~Hz}, 1 \mathrm{H}), 7.46(\mathrm{~d}, J=7.9 \mathrm{~Hz}, 1 \mathrm{H}), 7.40(\mathrm{td}, J=7.6,1.2 \mathrm{~Hz}, 1 \mathrm{H}), 7.04-7.01(\mathrm{~m}, 2 \mathrm{H})$, $6.92(\mathrm{~d}, J=7.8 \mathrm{~Hz}, 1 \mathrm{H}), 6.03(\mathrm{~s}, 2 \mathrm{H}) ;{ }^{13} \mathrm{C}\left\{{ }^{1} \mathrm{H}\right\} \mathrm{NMR}\left(125 \mathrm{MHz}, \mathrm{CDCl}_{3}\right): \delta 148.3,148.1,145.3,133.9$, 132.9, 132.2, 130.1, 127.4, 122.9, 119.4, 111.3, 109.3, 108.7, 101.6 ppm. Characterization data matched those reported in the literature. ${ }^{47}$

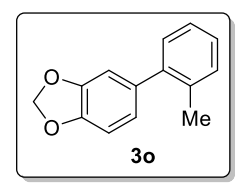

5-(o-tolyl)benzo $[d][1,3]$ dioxole (3o) $[$ CAS: 398470-00-3] was prepared following the general procedure A using $1 \mathbf{d}(135 \mathrm{mg}, 0.50 \mathrm{mmol})$ and $2 \mathrm{I}(131 \mathrm{mg}, 0.50 \mathrm{mmol})$ as starting materials. After stirring at $40{ }^{\circ} \mathrm{C}$ for $24 \mathrm{~h}$, the reaction was quenched following general procedure and the crude material was purified by flash chromatography on silica gel (hexanes/ethyl acetate $=100 / 1$ to $20 / 1$ ) to afford the desired product (80.3 mg, 75\%) as a white solid. ${ }^{1} \mathrm{H}$ NMR (400 MHz, $\left.\mathrm{CDCl}_{3}, \mathrm{TMS}\right): \delta 7.25-7.18$ (m, $\left.4 \mathrm{H}\right), 6.85$ (d, $J=$ $7.9 \mathrm{~Hz}, 1 \mathrm{H}), 6.80(\mathrm{~d}, J=1.7 \mathrm{~Hz}, 1 \mathrm{H}), 6.76(\mathrm{dd}, J=7.9,1.7 \mathrm{~Hz}, 1 \mathrm{H}), 5.99(\mathrm{~s}, 2 \mathrm{H}), 2.27(\mathrm{~s}, 3 \mathrm{H}) ;{ }^{13} \mathrm{C}\left\{{ }^{1} \mathrm{H}\right\}$ NMR (125 MHz, $\left.\mathrm{CDCl}_{3}\right): \delta 147.4,146.6,141.7,136.0,135.6,130.5,130.0,127.3,125.9,122.7,110.0$, 108.2, 101.2, $20.7 \mathrm{ppm}$. Characterization data matched those reported in the literature. ${ }^{48}$

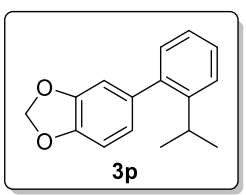

5-(2-isopropylphenyl)benzo[d][1,3]dioxole (3p) was prepared following the general procedure A using 1d (135 mg, $0.50 \mathrm{mmol})$ and $\mathbf{2 m}(218 \mathrm{mg}, 0.75 \mathrm{mmol})$ as starting materials. After stirring at $60{ }^{\circ} \mathrm{C}$ for 24 $\mathrm{h}$, the reaction was quenched following general procedure and the crude material was purified by flash chromatography on silica gel (hexanes/ethyl acetate $=100 / 1$ to $20 / 1)$ to afford the desired product $(83.0$ mg, 69\%) as a colorless oil. ${ }^{1} \mathrm{H}$ NMR (500 MHz, $\left.\mathrm{CDCl}_{3}\right)$ : $\delta 7.39-7.34(\mathrm{~m}, 2 \mathrm{H}), 7.22-7.16$ (m, $\left.2 \mathrm{H}\right), 6.87$ 
$(\mathrm{d}, J=7.9 \mathrm{~Hz}, 1 \mathrm{H}), 6.80(\mathrm{~d}, J=1.6 \mathrm{~Hz}, 1 \mathrm{H}), 6.76(\mathrm{dd}, J=7.9,1.6 \mathrm{~Hz}, 1 \mathrm{H}), 6.02(\mathrm{~s}, 2 \mathrm{H}), 3.11$ (hept, $J=$ $6.9 \mathrm{~Hz}, 1 \mathrm{H}), 1.18(\mathrm{~d}, J=6.9 \mathrm{~Hz}, 6 \mathrm{H}) ;{ }^{13} \mathrm{C}\left\{{ }^{1} \mathrm{H}\right\} \mathrm{NMR}\left(125 \mathrm{MHz}, \mathrm{CDCl}_{3}\right): \delta 147.4,146.7,146.6,140.8$, 136.1, 130.2, 127.8, 125.7, 125.4, 122.7, 110.1, 108.1, 101.2, 29.5, 24.4 ppm. HRMS (ESI) m/z calcd for $[\mathrm{M}+\mathrm{H}]^{+}$241.1223, found 241.1220. IR $\left(\mathrm{cm}^{-1}\right): 3018,1502,1220,1039,907,730$.

5-(2-isopropylphenyl)benzo[d][1,3]dioxole (3p) was also prepared following the general procedure $\mathrm{B}$ using $1 \mathbf{d}(135 \mathrm{mg}, 0.50 \mathrm{mmol})$ and $\mathbf{2 m}(218 \mathrm{mg}, 0.75 \mathrm{mmol})$ as starting materials. After stirring at $60{ }^{\circ} \mathrm{C}$ for $24 \mathrm{~h}$, the reaction was quenched following general procedure and the crude material was purified by flash chromatography on silica gel (hexanes/ethyl acetate $=100 / 1$ to 20/1) to afford the desired product (96.2 mg, 80\%) as a colorless oil. ${ }^{1} \mathrm{H}$ NMR (400 MHz, $\left.\mathrm{CDCl}_{3}\right): \delta 7.39-7.32$ (m, $\left.2 \mathrm{H}\right), 7.22-7.15$ (m, 2 H), $6.87(\mathrm{~d}, J=7.9 \mathrm{~Hz}, 1 \mathrm{H}), 6.79(\mathrm{~d}, J=1.6 \mathrm{~Hz}, 1 \mathrm{H}), 6.75(\mathrm{dd}, J=7.9,1.7 \mathrm{~Hz}, 1 \mathrm{H}), 6.01(\mathrm{~s}, 2 \mathrm{H}), 3.10$ (hept, $J=6.9 \mathrm{~Hz}, 1 \mathrm{H}), 1.18(\mathrm{~d}, J=6.9 \mathrm{~Hz}, 6 \mathrm{H}) ;{ }^{13} \mathrm{C}\left\{{ }^{1} \mathrm{H}\right\} \mathrm{NMR}\left(100 \mathrm{MHz}, \mathrm{CDCl}_{3}\right)$ : 147.4, 146.7, 146.6, $140.8,136.1,130.2,127.8,125.7,125.4,122.7,110.1,108.1,101.2,29.5,24.5$ ppm.

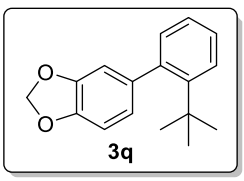

5-(2-(tert-butyl)phenyl)benzo[d][1,3]dioxole (3q) was prepared following the general procedure A using 1d (135 mg, $0.50 \mathrm{mmol})$ and $2 \mathrm{n}(228 \mathrm{mg}, 0.75 \mathrm{mmol})$ as starting materials. After stirring at $60{ }^{\circ} \mathrm{C}$ for $24 \mathrm{~h}$, the reaction was quenched following general procedure and the crude material was purified by flash chromatography on silica gel (hexanes/ethyl acetate $=100 / 1$ to $20 / 1)$ to afford the desired product $(69.2$ mg, 54\%) as a white solid. $\mathrm{mp}=70-72{ }^{\circ} \mathrm{C} .{ }^{1} \mathrm{H}$ NMR $\left(500 \mathrm{MHz}, \mathrm{CDCl}_{3}\right): \delta 7.53(\mathrm{~d}, J=6.7 \mathrm{~Hz}, 1 \mathrm{H}), 7.30$ $(\mathrm{td}, J=8.3,7.8,1.6 \mathrm{~Hz}, 1 \mathrm{H}), 7.17(\mathrm{td}, J=8.3,7.8,1.6 \mathrm{~Hz}, 1 \mathrm{H}), 7.02(\mathrm{dd}, J=7.3,1.5 \mathrm{~Hz}, 2 \mathrm{H}), 6.81-$ $6.76(\mathrm{~m}, 2 \mathrm{H}), 6.72(\mathrm{dd}, J=7.8,1.6 \mathrm{~Hz}, 1 \mathrm{H}), 6.01(\mathrm{~s}, 2 \mathrm{H}), 1.23(\mathrm{~s}, 9 \mathrm{H}) .{ }^{13} \mathrm{C}\left\{{ }^{1} \mathrm{H}\right\} \mathrm{NMR}\left(125 \mathrm{MHz}, \mathrm{CDCl}_{3}\right)$ : $\delta 148.2,146.7,146.3,141.7,139.1,133.0,127.4,126.9,125.1,123.4,111.1,107.3,101.1,36.8,32.8$ ppm. HRMS (ESI) $\mathrm{m} / \mathrm{z}$ calcd for $[\mathrm{M}+\mathrm{H}]^{+}$255.1380, found 255.1377. IR $\left(\mathrm{cm}^{-1}\right): 2953,2902,1476,1439,1241$, 1216, 1039, 757.

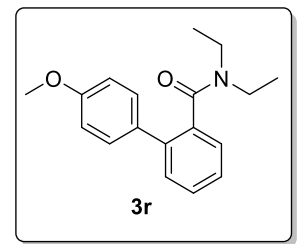

$N, N$-diethyl-4'-methoxy-[1,1'-biphenyl]-2-carboxamide (3r) [CAS: 1415045-57-6] was prepared following the general procedure A using $\mathbf{1 a}(128 \mathrm{mg}, 0.50 \mathrm{mmol})$ and $\mathbf{2 0}(260 \mathrm{mg}, 0.75 \mathrm{mmol})$ as starting 
materials. After stirring at $60{ }^{\circ} \mathrm{C}$ for $24 \mathrm{~h}$, the reaction was quenched following general procedure and the crude material was purified by flash chromatography on silica gel (hexanes/ethyl acetate $=5 / 1$ to $3 / 1$ ) to afford the desired product (116.3 mg, 82\%) as a white solid. ${ }^{1} \mathrm{H}$ NMR (500 M, $\left.\mathrm{CDCl}_{3}\right): \delta 7.42-7.37$ (m, 3 H), $7.36(\mathrm{~s}, 1 \mathrm{H}), 7.33(\mathrm{~m}, 2 \mathrm{H}), 6.90(\mathrm{~d}, J=8.7 \mathrm{~Hz}, 2 \mathrm{H}), 3.81(\mathrm{~s}, 3 \mathrm{H}), 3.73(\mathrm{~m}, 1 \mathrm{H}), 3.03(\mathrm{~m}, 1 \mathrm{H}), 2.93$ $(\mathrm{m}, 1 \mathrm{H}), 2.66(\mathrm{~m}, 1 \mathrm{H}), 0.93(\mathrm{t}, J=7.1 \mathrm{~Hz}, 3 \mathrm{H}), 0.73(\mathrm{t}, J=7.1 \mathrm{~Hz}, 3 \mathrm{H}) ;{ }^{13} \mathrm{C}\left\{{ }^{1} \mathrm{H}\right\} \mathrm{NMR}\left(125 \mathrm{M}, \mathrm{CDCl}_{3}\right)$ : $\delta 170.9,159.4,138.1,136.4,132.5,130.1,129.4,129.0,127.2,127.1,113.8,55.4,42.4,38.5,13.5,12.3$ ppm. Characterization data matched those reported in the literature. ${ }^{49}$

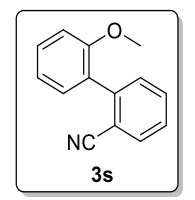

2'-methoxy-[1,1'-biphenyl]-2-carbonitrile (3s) [CAS: 150766-96-4] was prepared following the general procedure A using 11 (128 $\mathrm{mg}, 0.50 \mathrm{mmol})$ and $\mathbf{2 h}(205 \mathrm{mg}, 0.75 \mathrm{mmol})$ as starting materials. After stirring at $60{ }^{\circ} \mathrm{C}$ for $24 \mathrm{~h}$, the reaction was quenched following general procedure and the crude material was purified by flash chromatography on silica gel (hexanes/ethyl acetate $=10 / 1$ to $5 / 1$ ) to afford the desired product $(63.7 \mathrm{mg}, 61 \%)$ as a white solid. ${ }^{1} \mathrm{H} \mathrm{NMR}\left(500 \mathrm{MHz}, \mathrm{CDCl}_{3}, \mathrm{TMS}\right) \delta 7.71(\mathrm{dd}, J=7.8,1.4 \mathrm{~Hz}, 1$ H), $7.61(\mathrm{td}, J=7.7,1.4 \mathrm{~Hz}, 1 \mathrm{H}), 7.44(\mathrm{~d}, J=7.9 \mathrm{~Hz}, 1 \mathrm{H}), 7.40(\mathrm{td}, J=8.1,1.9 \mathrm{~Hz}, 2 \mathrm{H}), 7.25(\mathrm{dd}, J=$ 7.4, $1.7 \mathrm{~Hz}, 1 \mathrm{H}), 7.05(\mathrm{td}, J=7.5,1.0 \mathrm{~Hz}, 1 \mathrm{H}), 7.02(\mathrm{~d}, J=7.4 \mathrm{~Hz}, 1 \mathrm{H}), 3.83(\mathrm{~s}, 3 \mathrm{H}) ;{ }^{13} \mathrm{C}\left\{{ }^{1} \mathrm{H}\right\} \mathrm{NMR}$ $\left(100 \mathrm{M}, \mathrm{CDCl}_{3}\right): \delta 156.6,142.7,132.9,132.5,131.0,131.0,130.5,127.5,127.4,120.9,118.8,113.5,111.4$, $55.6 \mathrm{ppm}$. Characterization data matched those reported in the literature. ${ }^{50}$

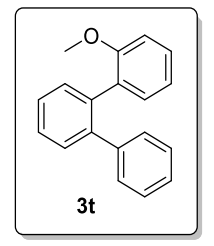

2-methoxy-1,1':2',1"'-terphenyl (3t) [CAS: 54113-08-5] was prepared following the general procedure A using 11 (128 mg, $0.50 \mathrm{mmol})$ and $\mathbf{2 p}(243 \mathrm{mg}, 0.75 \mathrm{mmol})$ as starting materials. After stirring at $60{ }^{\circ} \mathrm{C}$ for $24 \mathrm{~h}$, the reaction was quenched following general procedure and the crude material was purified by flash chromatography on silica gel (hexanes/ethyl acetate $=50 / 1$ to $10 / 1$ ) to afford the desired product (74.0 mg, 57\%) as a white solid. ${ }^{1} \mathrm{H}$ NMR (400 MHz, $\mathrm{CDCl}_{3}$, TMS): $\delta 7.41(\mathrm{~m}, 2 \mathrm{H}), 7.38$ (m, $\left.2 \mathrm{H}\right), 7.23$ - 7.11 (m, $7 \mathrm{H}), 6.91(\mathrm{td}, J=7.4,1.1 \mathrm{~Hz}, 1 \mathrm{H}), 6.68(\mathrm{~d}, J=9.2 \mathrm{~Hz}, 1 \mathrm{H}), 3.33(\mathrm{~s}, 3 \mathrm{H}) ;{ }^{13} \mathrm{C}\left\{{ }^{1} \mathrm{H}\right\} \mathrm{NMR}(125 \mathrm{MHz}$, $\left.\mathrm{CDCl}_{3}\right): \delta 156.4,142.3,141.8,137.4,131.8,131.1,130.8,129.8,129.1,128.7,127.7,127.6,127.3,126.4$, $120.5,110.8,55.1 \mathrm{ppm}$. Characterization data matched those reported in the literature. ${ }^{51}$ 


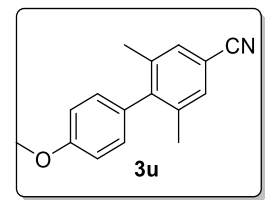

4'-methoxy-2,6-dimethyl-[1,1'-biphenyl]-4-carbonitrile (3u) was prepared following the general procedure A using $\mathbf{1 a}(128 \mathrm{mg}, 0.50 \mathrm{mmol})$ and $\mathbf{2 q}(226 \mathrm{mg}, 0.75 \mathrm{mmol})$ as starting materials. After stirring at $60{ }^{\circ} \mathrm{C}$ for $24 \mathrm{~h}$, the reaction was quenched following general procedure and the crude material was purified by flash chromatography on silica gel (hexanes/ethyl acetate $=20 / 1$ to $5 / 1$ ) to afford the desired product $(67.9 \mathrm{mg}, 57 \%)$ as a white solid. $\mathrm{mp}=118-120{ }^{\circ} \mathrm{C} .{ }^{1} \mathrm{H} \mathrm{NMR}\left(500 \mathrm{MHz}, \mathrm{CDCl}_{3}\right): 7.38(\mathrm{~s}, 2 \mathrm{H})$, $7.02-6.97$ (m, $4 \mathrm{H}), 3.86$ (s, $3 \mathrm{H}), 2.05(\mathrm{~s}, 6 \mathrm{H}) ;{ }^{13} \mathrm{C}\left\{{ }^{1} \mathrm{H}\right\} \mathrm{NMR}\left(125 \mathrm{MHz}, \mathrm{CDCl}_{3}\right): \delta$ 159.0, 146.7, 138.2, 131.5, 130.8, 129.5, 119.4, 114.3, 110.7, 55.4, 20.9 ppm. HRMS (ESI) m/z calcd for $[\mathrm{M}+\mathrm{H}]^{+} 238.1226$, found 238.1222. IR $\left(\mathrm{cm}^{-1}\right): 2965,2837,2221,1610,1516,1288,1242,883$.

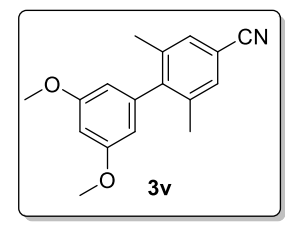

3',5'-dimethoxy-2,6-dimethyl-[1,1'-biphenyl]-4-carbonitrile (3v) was prepared following the general procedure A using 1c (143 mg, $0.50 \mathrm{mmol})$ and $\mathbf{2 q}(226 \mathrm{mg}, 0.75 \mathrm{mmol})$ as starting materials. After stirring at $60{ }^{\circ} \mathrm{C}$ for $24 \mathrm{~h}$, the reaction was quenched following general procedure and the crude material was purified by flash chromatography on silica gel (hexanes/ethyl acetate $=20 / 1$ to $5 / 1$ ) to afford the desired product $(73.0 \mathrm{mg}, 55 \%)$ as a white solid. $\mathrm{mp}=164-166{ }^{\circ} \mathrm{C} .{ }^{1} \mathrm{H} \mathrm{NMR}\left(500 \mathrm{MHz}, \mathrm{CDCl}_{3}\right): 7.38(\mathrm{~s}, 2 \mathrm{H})$, $6.47(\mathrm{t}, J=2.3 \mathrm{~Hz}, 1 \mathrm{H}), 6.23(\mathrm{~d}, J=2.3 \mathrm{~Hz}, 2 \mathrm{H}), 3.80(\mathrm{~s}, 6 \mathrm{H}), 2.09(\mathrm{~s}, 6 \mathrm{H}) ;{ }^{13} \mathrm{C}\left\{{ }^{1} \mathrm{H}\right\} \mathrm{NMR}(125 \mathrm{MHz}$, $\left.\mathrm{CDCl}_{3}\right): \delta 161.4,146.8,141.4,137.7,130.8,119.4,110.9,106.4,99.3,55.6,20.6$ ppm. HRMS (ESI) m/z calcd for $\left[\mathrm{M}+\mathrm{NH}_{4}\right]^{+}$285.1598, found 285.1594. IR $\left(\mathrm{cm}^{-1}\right): 2937,2836,2222,1590,1454,1204,1154$, $1028,836$.

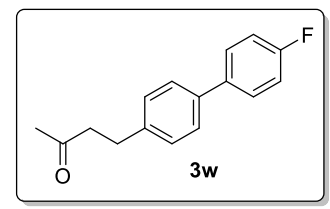

4-(4'-fluoro-[1,1'-biphenyl]-4-yl)butan-2-one (3w) [CAS: 1369389-99-0] was prepared following the general procedure A using $1 \mathbf{k}(148 \mathrm{mg}, 0.50 \mathrm{mmol})$ and $2 \mathbf{c}(133 \mathrm{mg}, 0.50 \mathrm{mmol})$ as starting materials. After stirring at $40{ }^{\circ} \mathrm{C}$ for $24 \mathrm{~h}$, the reaction was quenched following general procedure and the crude material was purified by flash chromatography on silica gel (hexanes/ethyl acetate $=20 / 1$ to $5 / 1$ ) to afford the desired 
product $(88.0 \mathrm{mg}, 73 \%)$ as a white solid. $\mathrm{mp}=64-66{ }^{\circ} \mathrm{C} .{ }^{1} \mathrm{H} \mathrm{NMR}\left(400 \mathrm{MHz}, \mathrm{CDCl}_{3}, \mathrm{TMS}\right) \delta 7.53-7.47$ (m, $2 \mathrm{H}), 7.44(\mathrm{~d}, J=8.2 \mathrm{~Hz}, 2 \mathrm{H}), 7.24(\mathrm{~d}, J=8.0 \mathrm{~Hz}, 2 \mathrm{H}), 7.09$ (t, $J=8.7 \mathrm{~Hz}, 2 \mathrm{H}), 2.92(\mathrm{t}, J=7.5 \mathrm{~Hz}$, $2 \mathrm{H}), 2.78(\mathrm{t}, J=7.5 \mathrm{~Hz}, 2 \mathrm{H}), 2.14(\mathrm{~s}, 3 \mathrm{H}) ;{ }^{13} \mathrm{C}\left\{{ }^{1} \mathrm{H}\right\} \mathrm{NMR}\left(100 \mathrm{MHz}, \mathrm{CDCl}_{3}\right) \delta 207.9,162.5(\mathrm{~d}, J=246.1$ $\mathrm{Hz}), 140.3,138.2,137.2$ (d, $J=3.2 \mathrm{~Hz}), 128.9,128.6$ (d, $J=8.0 \mathrm{~Hz}), 127.2,115.7$ (d, $J=21.4 \mathrm{~Hz}), 45.1$, 30.2, 29.4; ${ }^{19} \mathrm{~F}\left\{{ }^{1} \mathrm{H}\right\}$-NMR (376 MHz, $\left.\mathrm{CDCl}_{3}\right) \delta-115.94 \mathrm{ppm}$. HRMS (ESI) m/z calcd for $\left[\mathrm{M}+\mathrm{NH}_{4}\right]^{+}$ 260.1445, found 260.1441. IR ( $\left.\mathrm{cm}^{-1}\right): 2955,1715,1497,1373,1227,1159,812$.

4-(4'-fluoro-[1,1'-biphenyl]-4-yl)butan-2-one (3w) [CAS: 1369389-99-0] was prepared following the general procedure B using $1 \mathbf{k}(148 \mathrm{mg}, 0.50 \mathrm{mmol})$ and $\mathbf{2 c}(133 \mathrm{mg}, 0.50 \mathrm{mmol})$ as starting materials. After stirring at $40{ }^{\circ} \mathrm{C}$ for $24 \mathrm{~h}$, the reaction was quenched following general procedure and the crude material was purified by flash chromatography on silica gel (hexanes/ethyl acetate $=20 / 1$ to $5 / 1$ ) to afford the desired product (84.5 mg, 70\%) as a white solid. ${ }^{1} \mathrm{H}$ NMR (400 MHz, $\left.\mathrm{CDCl}_{3}, \mathrm{TMS}\right) \delta 7.55-7.47$ (m, $2 \mathrm{H}$ ), 7.44 $(\mathrm{d}, J=8.0 \mathrm{~Hz}, 2 \mathrm{H}), 7.23(\mathrm{~d}, J=7.8 \mathrm{~Hz}, 2 \mathrm{H}), 7.09$ (t, $J=8.7 \mathrm{~Hz}, 2 \mathrm{H}), 2.92(\mathrm{t}, J=7.5 \mathrm{~Hz}, 2 \mathrm{H}), 2.78(\mathrm{t}, J$ $=7.5 \mathrm{~Hz}, 2 \mathrm{H}), 2.14(\mathrm{~s}, 3 \mathrm{H}) ;{ }^{13} \mathrm{C}\left\{{ }^{1} \mathrm{H}\right\} \mathrm{NMR}\left(100 \mathrm{MHz}, \mathrm{CDCl}_{3}\right) \delta 207.9,162.4(\mathrm{~d}, J=246.0 \mathrm{~Hz}), 140.3$, 138.2, 137.1 (d, $J=3.2 \mathrm{~Hz}), 128.9,128.6$ (d, $J=8.0 \mathrm{~Hz}), 127.2,115.7$ (d, $J=21.3 \mathrm{~Hz}), 45.1,30.12,29.4$; ${ }^{19} \mathrm{~F}\left\{{ }^{1} \mathrm{H}\right\}-\mathrm{NMR}\left(376 \mathrm{MHz}, \mathrm{CDCl}_{3}\right) \delta-115.93 \mathrm{ppm}$.

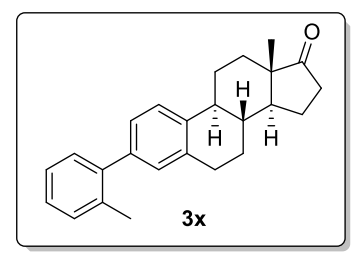

\section{(8R,9S,13S,14S)-13-methyl-3-(o-tolyl)-6,7,8,9,11,12,13,14,15,16-decahydro-17H-}

cyclopenta[a]phenanthren-17-one (3x) was prepared following the general procedure A using $\mathbf{1 m}$ (201 $\mathrm{mg}, 0.50 \mathrm{mmol})$ and $2 \mathrm{l}(131 \mathrm{mg}, 0.50 \mathrm{mmol})$ as starting materials. After stirring at $40{ }^{\circ} \mathrm{C}$ for $24 \mathrm{~h}$, the reaction was quenched following general procedure and the crude material was purified by flash chromatography on silica gel (hexanes/ethyl acetate $=20 / 1$ to $5 / 1$ ) to afford the desired product (137.3 $\mathrm{mg}$, $80 \%$ ) as a white solid. $\mathrm{mp}=204-206{ }^{\circ} \mathrm{C} .{ }^{1} \mathrm{H} \mathrm{NMR}\left(500 \mathrm{MHz}, \mathrm{CDCl}_{3}\right.$, TMS) $\delta 7.32(\mathrm{~d}, J=7.9 \mathrm{~Hz}, 1 \mathrm{H})$, $7.27-7.20(\mathrm{~m}, 4 \mathrm{H}), 7.12(\mathrm{~d}, J=6.0 \mathrm{~Hz}, 1 \mathrm{H}), 7.06(\mathrm{~s}, 1 \mathrm{H}), 2.95(\mathrm{~m}, 2 \mathrm{H}), 2.56-2.43(\mathrm{~m}, 2 \mathrm{H}), 2.36(\mathrm{td}$, $J=11.0,4.0 \mathrm{~Hz}, 1 \mathrm{H}), 2.29$ (s, $3 \mathrm{H}), 2.20-2.02(\mathrm{~m}, 3 \mathrm{H}), 1.99$ (d, $J=12.6 \mathrm{~Hz}, 1 \mathrm{H}), 1.73-1.43$ (m, $6 \mathrm{H})$, $0.93(\mathrm{~s}, 3 \mathrm{H}) ;{ }^{13} \mathrm{C}\left\{{ }^{1} \mathrm{H}\right\}-\mathrm{NMR}\left(125 \mathrm{MHz}, \mathrm{CDCl}_{3}\right)$ : $\delta 221.0,141.8,139.6,138.4,136.3,135.5,130.4,130.0$, 129.9, 127.2, 126.8, 125.9, 125.2, 50.7, 48.2, 44.6, 38.3, 36.0, 31.8, 29.6, 26.7, 25.9, 21.8, 20.7, 14.0 ppm. HRMS (ESI) $\mathrm{m} / \mathrm{z}$ calcd for $[\mathrm{M}+\mathrm{H}]^{+} 345.2213$, found 345.2212. IR $\left(\mathrm{cm}^{-1}\right): 2927,2858,1736,1480,1453$, $1155,1006,757,732$. 


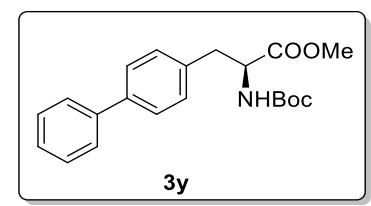

Methyl (S)-3-([1,1'-biphenyl]-4-yl)-2-((tert-butoxycarbonyl)amino)propanoate (3y) [CAS: 13725596-8] was prepared following the general procedure A using $\mathbf{1 n}(213 \mathrm{mg}, 0.50 \mathrm{mmol})$ and $\mathbf{2 a}$ (124 $\mathrm{mg}$, $0.50 \mathrm{mmol}$ ) as starting materials. After stirring at $40{ }^{\circ} \mathrm{C}$ for $24 \mathrm{~h}$, the reaction was quenched following general procedure and the crude material was purified by flash chromatography on silica gel (hexanes/ethyl acetate $=20 / 1$ to $3 / 1)$ to afford the desired product $(120.5 \mathrm{mg}, 68 \%)$ as a white solid. ${ }^{1} \mathrm{H}$ NMR (400 MHz, $\left.\mathrm{CDCl}_{3}\right): 7.56(\mathrm{dd}, J=18.6,7.8 \mathrm{~Hz}, 4 \mathrm{H}), 7.44(\mathrm{t}, J=7.6 \mathrm{~Hz}, 2 \mathrm{H}), 7.34(\mathrm{t}, J=7.5 \mathrm{~Hz}, 1 \mathrm{H}), 7.21(\mathrm{~d}, J=7.8$ Hz, 2 H), 5.05 (d, J = 8.4 Hz, 1 H), 4.64 (q, $J=6.7$ Hz, 1 H), 3.74 (s, 3 H), $3.22-3.05$ (m, 2 H), 1.43 (s, 9 $\mathrm{H}) ;{ }^{13} \mathrm{C}\left\{{ }^{1} \mathrm{H}\right\} \mathrm{NMR}\left(100 \mathrm{MHz}, \mathrm{CDCl}_{3}\right): \delta 172.5,155.3,140.9,140.0,135.2,129.9,128.9,127.4,127.2,80.1$, 54.5, 52.4, 38.1, $28.4 \mathrm{ppm} .{ }^{1} \mathrm{H}$ NMR data matched those reported in the literature, ${ }^{52}{ }^{13} \mathrm{C}\left\{{ }^{1} \mathrm{H}\right\}$ NMR data were not reported previously. The ${ }^{13} \mathrm{C}$ NMR spectrum is missing one signal due to the overlap of two signals. Similar peak overlap was reported in the ${ }^{13} \mathrm{C}$ NMR data of methyl 3-([1,1'-biphenyl]-4-yl)-2-((tertbutoxycarbonyl)amino)propanoate. ${ }^{53}$

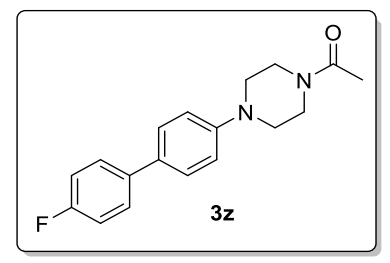

1-(4-(4'-fluoro-[1,1'-biphenyl]-4-yl)piperazin-1-yl)ethan-1-one (3z) was prepared following the general procedure A using $10(176 \mathrm{mg}, 0.50 \mathrm{mmol})$ and $2 \mathbf{c}(133 \mathrm{mg}, 0.50 \mathrm{mmol})$ as starting materials. After stirring at $40{ }^{\circ} \mathrm{C}$ for $24 \mathrm{~h}$, the reaction was quenched following general procedure and the crude material was purified by flash chromatography on silica gel (hexanes/ethyl acetate $=5 / 1$ to $100 \%$ ethyl acetate) to afford the desired product $(97.0 \mathrm{mg}, 65 \%)$ as a white solid. $\mathrm{mp}=188-190{ }^{\circ} \mathrm{C} .{ }^{1} \mathrm{H}$ NMR $\left(500 \mathrm{MHz}, \mathrm{DMSO}-\mathrm{d}_{6}\right)$ : $\delta 7.63(\mathrm{dd}, J=8.7,5.5 \mathrm{~Hz}, 2 \mathrm{H}), 7.52(\mathrm{~d}, J=8.8 \mathrm{~Hz}, 2 \mathrm{H}), 7.23(\mathrm{t}, J=8.9 \mathrm{~Hz}, 2 \mathrm{H}), 7.04$ (d, $J=8.8 \mathrm{~Hz}, 2$ $\mathrm{H}), 3.58(\mathrm{q}, J=5.9 \mathrm{~Hz}, 4 \mathrm{H}), 3.20(\mathrm{t}, J=5.2 \mathrm{~Hz}, 2 \mathrm{H}), 3.14(\mathrm{t}, J=5.3 \mathrm{~Hz}, 2 \mathrm{H}), 2.04(\mathrm{~s}, 3 \mathrm{H}) ;{ }^{13} \mathrm{C}\left\{{ }^{1} \mathrm{H}\right\}$ NMR (125 MHz, DMSO-d 6 ): $\delta 168.3,161.3(\mathrm{~d}, J=243.2 \mathrm{~Hz}), 150.1,136.5,129.8,127.7$ (d, $J=8.0 \mathrm{~Hz})$, 127.2, 116.0, $115.6(\mathrm{~d}, J=21.2 \mathrm{~Hz}), 48.4,48.0,45.4,21.2 ;{ }^{19} \mathrm{~F}\left\{{ }^{1} \mathrm{H}\right\}$ NMR (376 MHz, DMSO-d 6$): \delta-116.94$ ppm. HRMS (ESI) m/z calcd for $[\mathrm{M}+\mathrm{H}]^{+}$299.1554, found 299.1551. IR $\left(\mathrm{cm}^{-1}\right): 2828,1618,1501,1231$, $1001,810,558$. 


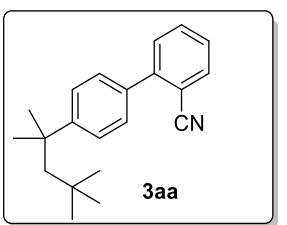

4'-(2,4,4-trimethylpentan-2-yl)-[1,1'-biphenyl]-2-carbonitrile (3aa) was prepared following the general procedure A using $\mathbf{1 p}(169 \mathrm{mg}, 0.50 \mathrm{mmol})$ and $\mathbf{2 h}(137 \mathrm{mg}, 0.50 \mathrm{mmol})$ as starting materials. After stirring at $40{ }^{\circ} \mathrm{C}$ for $24 \mathrm{~h}$, the reaction was quenched following general procedure and the crude material was purified by flash chromatography on silica gel (hexanes/ethyl acetate $=10 / 1$ to $5 / 1$ ) to afford the desired product (122.4 mg, 83\%) as a colorless oil. ${ }^{1} \mathrm{H} \mathrm{NMR}\left(500 \mathrm{MHz}, \mathrm{CDCl}_{3}\right): \delta 7.75(\mathrm{~d}, J=8.6 \mathrm{~Hz}, 1 \mathrm{H}), 7.62$ $(\mathrm{t}, J=7.0 \mathrm{~Hz}, 1 \mathrm{H}), 7.53(\mathrm{~d}, J=7.8 \mathrm{~Hz}, 1 \mathrm{H}), 7.50$ (s, $4 \mathrm{H}), 7.41(\mathrm{t}, J=7.6 \mathrm{~Hz}, 1 \mathrm{H}), 1.79(\mathrm{~s}, 2 \mathrm{H}), 1.42$ (s, $6 \mathrm{H}), 0.76(\mathrm{~s}, 9 \mathrm{H}) ;{ }^{13} \mathrm{C}\left\{{ }^{1} \mathrm{H}\right\} \mathrm{NMR}\left(125 \mathrm{MHz}, \mathrm{CDCl}_{3}\right): \delta 151.2,145.6,135.2,133.9,132.9,130.2,128.3$, 127.4, 126.7, 119.0, 111.4, 57.2, 38.8, 32.6, 32.0, $31.6 \mathrm{ppm}$. HRMS (ESI) m/z calcd for $[\mathrm{M}+\mathrm{H}]^{+} 292.2060$, found 292.2059. IR $\left(\mathrm{cm}^{-1}\right)$ : 2952, 2901, 2223, 1732, 1477, 1365, 835, 754.

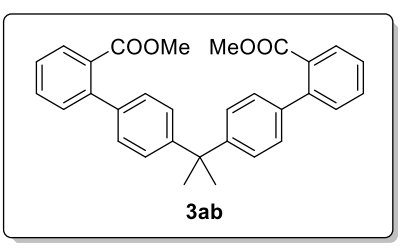

Dimethyl 4',4'"'-(propane-2,2-diyl)bis([1,1'-biphenyl]-2-carboxylate) (3ab) was prepared following the general procedure A using 1q $(123 \mathrm{mg}, 0.25 \mathrm{mmol})$ and $\mathbf{2 r}(153 \mathrm{mg}, 0.50 \mathrm{mmol})$ as starting materials. After stirring at $40{ }^{\circ} \mathrm{C}$ for $24 \mathrm{~h}$, the reaction was quenched following general procedure and the crude material was purified by flash chromatography on silica gel (hexanes/ethyl acetate $=10 / 1$ to $3 / 1$ ) to afford the desired product (68.9 mg, 59\%) as a colorless oil. ${ }^{1} \mathrm{H} \mathrm{NMR}\left(500 \mathrm{MHz}, \mathrm{CDCl}_{3}\right): \delta 7.80(\mathrm{dd}, J=8.2,1.4 \mathrm{~Hz}, 2 \mathrm{H})$, $7.52(\mathrm{td}, J=7.8,1.4 \mathrm{~Hz}, 2 \mathrm{H}), 7.40$ (d, $J=7.5 \mathrm{~Hz}, 4 \mathrm{H}), 7.30$ (d, $J=8.4 \mathrm{~Hz}, 4 \mathrm{H}), 7.24$ (d, $J=8.3 \mathrm{~Hz}, 4 \mathrm{H})$, $3.62(\mathrm{~s}, 6 \mathrm{H}), 1.77(\mathrm{~s}, 6 \mathrm{H}) ;{ }^{13} \mathrm{C}\left\{{ }^{1} \mathrm{H}\right\} \mathrm{NMR}\left(125 \mathrm{MHz}, \mathrm{CDCl}_{3}\right): \delta 169.5,149.8,142.3,138.7,131.4,131.2$, 130.8, 129.9, 128.2, 127.2, 126.7, 52.0, 42.9, $30.9 \mathrm{ppm}$. HRMS (ESI) $\mathrm{m} / \mathrm{z}$ calcd for $\left[\mathrm{M}+\mathrm{NH}_{4}\right]^{+} 482.2326$, found 482.2324. IR $\left(\mathrm{cm}^{-1}\right)$ : 2967, 1722, 1279, 1244, 1087, 839, 765, 745.

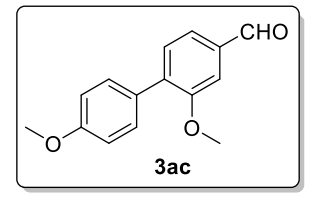

2,4'-dimethoxy-[1,1'-biphenyl]-4-carbaldehyde (3ac) [CAS: 2039147-37-8] was prepared following the general procedure A using $1 \mathrm{a}(128 \mathrm{mg}, 0.50 \mathrm{mmol})$ and $2 \mathrm{~s}(153 \mathrm{mg}, 0.50 \mathrm{mmol})$ as starting materials and $5.0 \mathrm{~mol} \% \mathrm{NiCl}_{2}$ (dme)/6.0 mol\% 4,4'-dimethoxy-2,2'-bipyridine and $5.0 \mathrm{~mol} \% \mathrm{PdCl}_{2} / 6.0 \mathrm{~mol} \% \mathrm{dppb}$ as 
catalysts. After stirring at $40{ }^{\circ} \mathrm{C}$ for $24 \mathrm{~h}$, the reaction was quenched following general procedure and the crude material was purified by flash chromatography on silica gel by flash chromatography on silica gel (hexanes/ethyl acetate $=10 / 1$ to $3 / 1$ ) afforded the desired product $\left(76.0 \mathrm{mg}, 64 \%\right.$ ) as a white solid. ${ }^{1} \mathrm{H}$ NMR (500 MHz, $\left.\mathrm{CDCl}_{3}\right): \delta 10.00$ (s, $\left.1 \mathrm{H}\right), 7.53-7.46(\mathrm{~m}, 5 \mathrm{H}), 6.98$ (d, J = 8.7 Hz, $\left.2 \mathrm{H}\right), 3.89$ (s, $\left.3 \mathrm{H}\right), 3.86$ (s, $3 \mathrm{H}) ;{ }^{13} \mathrm{C}\left\{{ }^{1} \mathrm{H}\right\} \mathrm{NMR}\left(125 \mathrm{MHz}, \mathrm{CDCl}_{3}\right): \delta 192.0,159.6,157.2,137.1,136.5,131.1,130.8,129.7,124.7$, $113.8,109.7,55.9,55.5 \mathrm{ppm}$. Characterization data matched those reported in the literature. ${ }^{54}$

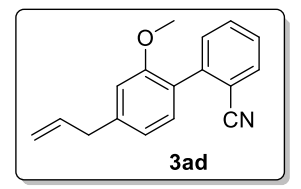

4'-allyl-2'-methoxy-[1,1'-biphenyl]-2-carbonitrile (3ad) was prepared following the general procedure A using $1 \mathbf{r}(148 \mathrm{mg}, 0.50 \mathrm{mmol})$ and $\mathbf{2 h}(205 \mathrm{mg}, 0.75 \mathrm{mmol})$ as starting materials. After stirring at $60{ }^{\circ} \mathrm{C}$ for $24 \mathrm{~h}$, the reaction was quenched following general procedure and the crude material was purified by flash chromatography on silica gel (hexanes/ethyl acetate $=10 / 1$ to 3/1) to afford the desired product (63.0 mg, 50\%) as a white solid. $\mathrm{mp}=59-61{ }^{\circ} \mathrm{C} .{ }^{1} \mathrm{H} \mathrm{NMR}\left(500 \mathrm{MHz}, \mathrm{CDCl}_{3}\right): \delta 7.72(\mathrm{~d}, J=7.7 \mathrm{~Hz}, 1 \mathrm{H}), 7.61$ $(\mathrm{t}, J=7.7 \mathrm{~Hz}, 1 \mathrm{H}), 7.45(\mathrm{~d}, J=7.7 \mathrm{~Hz}, 1 \mathrm{H}), 7.41(\mathrm{t}, J=7.6 \mathrm{~Hz}, 1 \mathrm{H}), 7.19(\mathrm{~d}, J=7.6 \mathrm{~Hz}, 1 \mathrm{H}), 6.90(\mathrm{~d}, J$ = $7.7 \mathrm{~Hz}, 1 \mathrm{H}), 6.86(\mathrm{~s}, 1 \mathrm{H}), 6.02(\mathrm{~m}, 1 \mathrm{H}), 5.21-5.10(\mathrm{~m}, 2 \mathrm{H}), 3.84(\mathrm{~s}, 3 \mathrm{H}), 3.46(\mathrm{~d}, J=6.7 \mathrm{~Hz}, 2 \mathrm{H})$; ${ }^{13} \mathrm{C}\left\{{ }^{1} \mathrm{H}\right\}$ NMR $\left(125 \mathrm{MHz}, \mathrm{CDCl}_{3}\right): \delta 156.6,142.9,142.7,137.0,133.0,132.5,131.1,131.0,127.4,125.3$, 121.1, 118.9, 116.5, 113.6, 111.8, 55.6, 40.6 ppm. HRMS (ESI) $\mathrm{m} / \mathrm{z}$ calcd for $\left[\mathrm{M}+\mathrm{NH}_{4}\right]^{+} 250.1226$, found 250.1223. IR $\left(\mathrm{cm}^{-1}\right): 3003,2910,2224,1610,1411,1162,1036,756,548$.

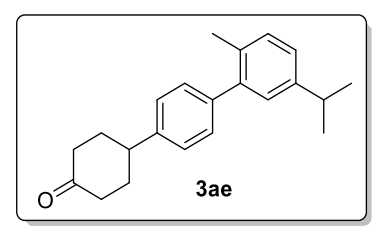

4-(2'-isopropyl-[1,1'-biphenyl]-4-yl)cyclohexan-1-one (3ae) was prepared following the general procedure A using $1 \mathrm{~s}(161 \mathrm{mg}, 0.50 \mathrm{mmol})$ and $2 \mathrm{t}(228 \mathrm{mg}, 0.75 \mathrm{mmol})$ as starting materials. After stirring at $40{ }^{\circ} \mathrm{C}$ for $24 \mathrm{~h}$, the reaction was quenched following general procedure and the crude material was purified by flash chromatography on silica gel (hexanes/ethyl acetate $=10 / 1$ to $5 / 1$ ) to afford the desired product $(72.0 \mathrm{mg}, 47 \%)$ as a white solid. $\mathrm{mp}=82-84{ }^{\circ} \mathrm{C} .{ }^{1} \mathrm{H} \mathrm{NMR}\left(500 \mathrm{MHz}, \mathrm{CDCl}_{3}\right): \delta 7.32-7.27(\mathrm{~m}$, $5 \mathrm{H}), 7.19(\mathrm{~d}, J=8.0 \mathrm{~Hz}, 1 \mathrm{H}), 7.03(\mathrm{~s}, 1 \mathrm{H}), 3.16-3.00(\mathrm{~m}, 2 \mathrm{H}), 2.60-2.50(\mathrm{~m}, 4 \mathrm{H}), 2.37$ (s, $3 \mathrm{H}), 2.37$ $-2.29(\mathrm{~m}, 2 \mathrm{H}), 2.10-1.97(\mathrm{~m}, 2 \mathrm{H}), 1.18(\mathrm{~d}, J=6.9 \mathrm{~Hz}, 6 \mathrm{H}) ;{ }^{13} \mathrm{C}\left\{{ }^{1} \mathrm{H}\right\} \mathrm{NMR}\left(125 \mathrm{MHz}, \mathrm{CDCl}_{3}\right): \delta 211.4$, 143.6, 143.2, 140.8, 140.6, 134.9, 130.9, 129.6, 128.6, 126.4, 125.7, 42.6, 41.6, 34.2, 29.1, 24.6, 21.1 ppm. 
HRMS (ESI) $\mathrm{m} / \mathrm{z}$ calcd for $[\mathrm{M}+\mathrm{H}]^{+}$307.2056, found 307.2053. IR $\left(\mathrm{cm}^{-1}\right): 2958,2865,1715,1493,1161$, 820.

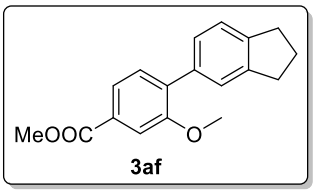

Methyl 4-(2,3-dihydro-1H-inden-5-yl)-3-methoxybenzoate (3af) was prepared following the general procedure A using $1 \mathbf{t}(133 \mathrm{mg}, 0.50 \mathrm{mmol})$ and $\mathbf{2 u}(168 \mathrm{mg}, 0.50 \mathrm{mmol})$ as starting materials. After stirring at $40{ }^{\circ} \mathrm{C}$ for $24 \mathrm{~h}$, the reaction was quenched following general procedure and the crude material was purified by flash chromatography on silica gel (hexanes/ethyl acetate $=10 / 1$ to $3 / 1$ ) to afford the desired product (117.0 mg, 83\%) as a colorless oil. ${ }^{1} \mathrm{H} \mathrm{NMR}\left(500 \mathrm{MHz}, \mathrm{CDCl}_{3}\right): \delta 7.72(\mathrm{dd}, J=7.8,1.5 \mathrm{~Hz}, 1 \mathrm{H})$, $7.66(\mathrm{~d}, J=1.4 \mathrm{~Hz}, 1 \mathrm{H}), 7.41(\mathrm{~s}, 1 \mathrm{H}), 7.38(\mathrm{~d}, J=7.8 \mathrm{~Hz}, 1 \mathrm{H}), 7.31(\mathrm{q}, J=7.8 \mathrm{~Hz}, 2 \mathrm{H}), 3.96(\mathrm{~s}, 3 \mathrm{H})$, 3.89 (s, $3 \mathrm{H}), 2.97$ (q, $J=7.1 \mathrm{~Hz}, 4 \mathrm{H}), 2.13(\mathrm{p}, J=7.4 \mathrm{~Hz}, 2 \mathrm{H}) ;{ }^{13} \mathrm{C}\left\{{ }^{1} \mathrm{H}\right\} \mathrm{NMR}\left(125 \mathrm{MHz}, \mathrm{CDCl}_{3}\right): \delta$ 167.2, 156.6, 144.3, 144.0, 136.1, 135.6, 130.9, 130.1, 127.6, 125.6, 124.2, 122.3, 112.0, 55.9, 52.3, 33.0, 32.9, $25.6 \mathrm{ppm}$. HRMS (ESI) $\mathrm{m} / \mathrm{z}$ calcd for $[\mathrm{M}+\mathrm{H}]^{+}$283.1329, found 283.1326. IR $\left(\mathrm{cm}^{-1}\right): 2949,2842$, $1718,1397,1286,1227,1109,1035,765$.

Methyl 4-(2,3-dihydro-1H-inden-5-yl)-3-methoxybenzoate (3af) was prepared following the general procedure B using $1 \mathbf{t}(133 \mathrm{mg}, 0.50 \mathrm{mmol})$ and $\mathbf{2 u}(168 \mathrm{mg}, 0.50 \mathrm{mmol})$ as starting materials. After stirring at $40{ }^{\circ} \mathrm{C}$ for $24 \mathrm{~h}$, the reaction was quenched following general procedure and the crude material was purified by flash chromatography on silica gel (hexanes/ethyl acetate $=10 / 1$ to $3 / 1$ ) to afford the desired product $(111.5 \mathrm{mg}, 79 \%)$ as a colorless oil. ${ }^{1} \mathrm{H} \mathrm{NMR}\left(400 \mathrm{MHz}, \mathrm{CDCl}_{3}\right) \delta 7.73(\mathrm{dd}, J=7.8,1.6 \mathrm{~Hz}, 1 \mathrm{H})$, $7.67(\mathrm{~d}, J=1.6 \mathrm{~Hz}, 1 \mathrm{H}), 7.43(\mathrm{~s}, 1 \mathrm{H}), 7.40$ (d, $J=7.8 \mathrm{~Hz}, 1 \mathrm{H}), 7.33$ (q, $J=7.8 \mathrm{~Hz}, 2 \mathrm{H}), 3.97(\mathrm{~s}, 3 \mathrm{H})$, 3.90 (s, $3 \mathrm{H}), 2.99$ (q, $J=6.7 \mathrm{~Hz}, 4 \mathrm{H}), 2.14(\mathrm{p}, J=7.4 \mathrm{~Hz}, 2 \mathrm{H}) ;{ }^{13} \mathrm{C}\left\{{ }^{1} \mathrm{H}\right\} \mathrm{NMR}\left(100 \mathrm{MHz}, \mathrm{CDCl}_{3}\right): \delta$ 167.1, 156.5, 144.3, 144.0, 136.0, 135.5, 130.9, 130.0, 127.6, 125.5, 124.2, 122.3, 112.0, 55.8, 52.3, 33.0, $32.8,25.6 \mathrm{ppm}$.

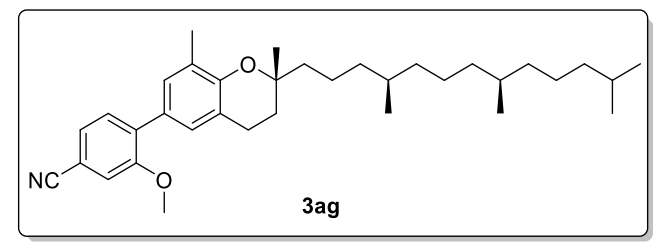

4-((R)-2,8-dimethyl-2-((4R,8R)-4,8,12-trimethyltridecyl)chroman-6-yl)-3-methoxybenzonitrile (3ag) was prepared following the general procedure A using $1 \mathbf{u}(267 \mathrm{mg}, 0.50 \mathrm{mmol})$ and $\mathbf{2 v}(152 \mathrm{mg}, 0.50 \mathrm{mmol})$ 
as starting materials. After stirring at $40{ }^{\circ} \mathrm{C}$ for $24 \mathrm{~h}$, the reaction was quenched following general procedure and the crude material was purified by flash chromatography on silica gel (hexanes/ethyl acetate $=100 / 1$ to $10 / 1)$ to afford the desired product $(137.2 \mathrm{mg}, 53 \%)$ as a colorless oil. ${ }^{1} \mathrm{H}$ NMR (500 MHz, $\left.\mathrm{CDCl}_{3}\right): 7.37$ $(\mathrm{d}, J=7.8 \mathrm{~Hz}, 1 \mathrm{H}), 7.30(\mathrm{dd}, J=7.8,1.5 \mathrm{~Hz}, 1 \mathrm{H}), 7.16(\mathrm{~d}, J=1.5 \mathrm{~Hz}, 1 \mathrm{H}), 7.14(\mathrm{~d}, J=2.7 \mathrm{~Hz}, 1 \mathrm{H})$, $7.08(\mathrm{~d}, J=2.2 \mathrm{~Hz}, 1 \mathrm{H}), 3.85(\mathrm{~s}, 3 \mathrm{H}), 2.79(\mathrm{~m}, 2 \mathrm{H}), 2.21$ (s, $3 \mathrm{H}), 1.56-1.47$ (m, $2 \mathrm{H}), 1.44$ - 1.32 (m, $5 \mathrm{H}), 1.31$ (s, $3 \mathrm{H}), 1.29-1.18(\mathrm{~m}, 6 \mathrm{H}), 1.17-1.12(\mathrm{~m}, 3 \mathrm{H}), 1.10-1.03$ (m, $3 \mathrm{H}), 0.88$ (d, J = 6.6 Hz, 9 $\mathrm{H}), 0.85(\mathrm{~d}, J=6.6 \mathrm{~Hz}, 3 \mathrm{H}) ;{ }^{13} \mathrm{C}\left\{{ }^{1} \mathrm{H}\right\}$ NMR $\left(125 \mathrm{MHz}, \mathrm{CDCl}_{3}\right): \delta$ 156.7, 152.6, 136.2, 131.3, 129.5, 128.2, 127.0, 126.3, 125.0, 120.4, 119.4, 114.2, 110.9, 76.7, 56.0, 40.5, 39.5, 37.62, 37.60, 37.4, 33.0, 32.9, 31.2, 28.2, 25.0, 24.6, 24.6, 22.9, 22.8, 22.6, 21.2, 19.9, 19.8, $16.4 \mathrm{ppm}$. HRMS (ESI) m/z calcd for $\left[\mathrm{M}+\mathrm{NH}_{4}\right]^{+}$ 535.4258, found 535.4259. IR ( $\left.\mathrm{cm}^{-1}\right):$ 2954, 2865, 2227, 1472, 1226, 1133, 1034, 823.

\section{Synthesis of compound 3w on $5.0 \mathrm{mmol}$ scale}

Scheme S9. Synthesis of compound $\mathbf{3 w}$ on $5.0 \mathrm{mmol}$ scale
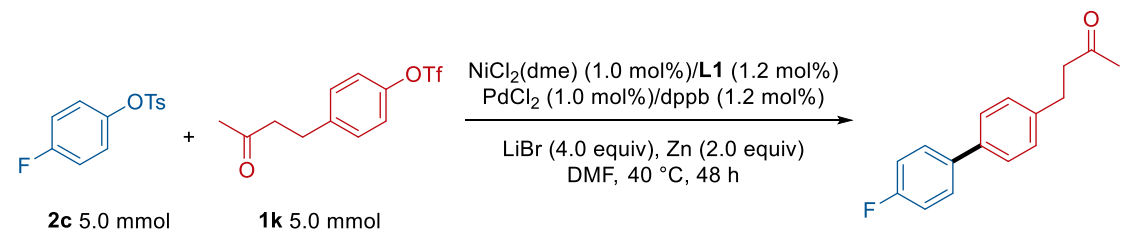

3w $823 \mathrm{mg}, 68 \%$

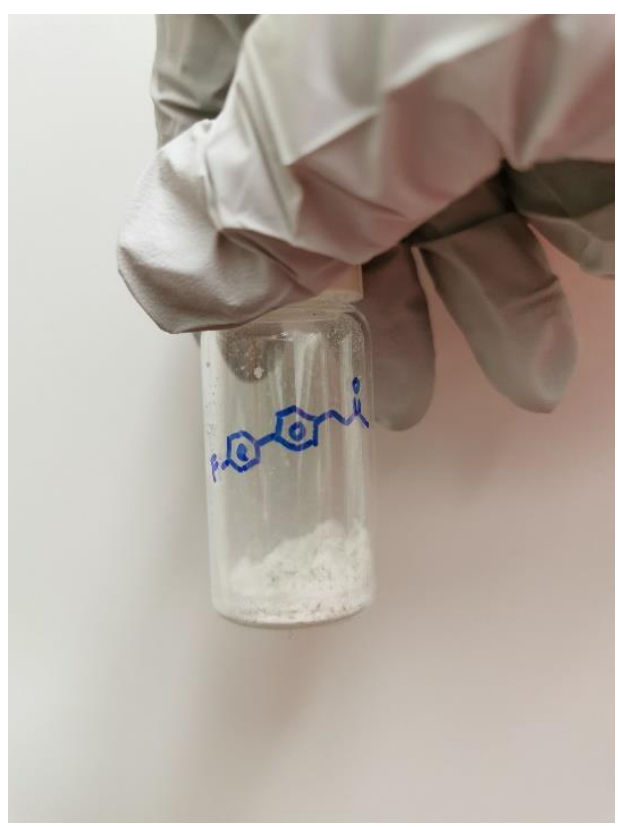

Figure S3. Compound 3w isolated from the reaction. 


\section{Procedure for synthesis of compound $3 \mathrm{w}$ on $5.0 \mathrm{mmol}$ scale}

The following procedures were conducted in a $\mathrm{N}_{2}$ filled glovebox for convenience. Nickel and palladium catalyst stock solutions were prepared following general procedure for optimization studies (page S4) using $\mathbf{L 1}$ (18.5 $\mathrm{mg}, 0.060 \mathrm{mmol}$ ) as the amine ligand and dppb (25.6 $\mathrm{mg}, 0.060 \mathrm{mmol}$ ) as the phosphine ligand.

\section{Catalytic Reaction Setup}

An oven-dried $100 \mathrm{~mL}$ Schlenk tube equipped with a PTFE-coated stir-bar was charged sequentially with 1k (1480 mg, $5.00 \mathrm{mmol}, 1.00$ equiv), 2c (1330 mg, $5.00 \mathrm{mmol}, 1.00$ equiv), $\mathrm{LiBr}$ (1740 mg, $20.0 \mathrm{mmol}, 4.00$ equiv), and Zn flake (650 mg, $10.0 \mathrm{mmol}, 2.00$ equiv). (Attention: zinc flake instead of zinc powder was used as the reductant.) Nickel catalyst stock solution (2.0 mL for $1.0 \mathrm{~mol} \%)$, palladium catalyst stock solution ( $2.0 \mathrm{~mL}$ for $1.0 \mathrm{~mol} \%)$ were added, followed by additional DMF (11.0 mL). The reaction tube was sealed with a PTFE valve plug, removed from glovebox, and the reaction mixture was stirred $(1000 \mathrm{rpm})$ at $\mathrm{rt}$ for $20 \mathrm{~min}$. The reaction tube was then placed into a $40{ }^{\circ} \mathrm{C}$ oil bath and stirred $(1000$ rpm) for $48 \mathrm{~h}$.

\section{Isolation and purification}

When the reaction was judged complete (GC analysis), the reaction mixture was cooled to rt, diluted with ethyl acetate $(50 \mathrm{~mL})$, filtered through a short plug of silica gel $(3.0 \mathrm{~cm} \times 3.0 \mathrm{~cm})$, and the vessel and silica gel were washed with extra portions of ethyl acetate $(50 \mathrm{~mL} \times 4)$. The combined filtrate was poured into a $500 \mathrm{~mL}$ separatory funnel and washed with brine $(50 \mathrm{~mL} \times 3)$. The ethyl acetate layer was set aside while the aqueous layer was washed further with ethyl acetate $(50 \mathrm{~mL} \times 3)$. The combined organic layers were dried over anhydrous $\mathrm{MgSO}_{4}$, the resulting mixture was filtered to remove the $\mathrm{MgSO}_{4}$, and the filtrate was concentrated by rotary evaporation. The crude material was purified by flash chromatography on silica gel (hexanes/ethyl acetate $=100 / 1$ to $10 / 1)$ to afford the desired product $(822.6$ mg, 68\%) as a white solid. ${ }^{1} \mathrm{H}$ NMR (500 MHz, $\mathrm{CDCl}_{3}$, TMS) $\delta 7.50(\mathrm{dd}, J=8.5,5.4 \mathrm{~Hz}, 2 \mathrm{H}), 7.44(\mathrm{~d}, J$ $=7.9 \mathrm{~Hz}, 2 \mathrm{H}), 7.23(\mathrm{~d}, J=7.8 \mathrm{~Hz}, 2 \mathrm{H}), 7.09(\mathrm{t}, J=8.6 \mathrm{~Hz}, 2 \mathrm{H}), 2.92(\mathrm{t}, J=7.6 \mathrm{~Hz}, 2 \mathrm{H}), 2.77(\mathrm{t}, J=7.6$ $\mathrm{Hz}, 2 \mathrm{H}), 2.14(\mathrm{~s}, 3 \mathrm{H}) ;{ }^{13} \mathrm{C}\left\{{ }^{1} \mathrm{H}\right\} \mathrm{NMR}\left(125 \mathrm{MHz}, \mathrm{CDCl}_{3}\right) \delta 207.8,162.5$ (d, J = 246.1 Hz), 140.3, 138.2, $137.2(\mathrm{~d}, J=3.3 \mathrm{~Hz}), 128.9,128.6(\mathrm{~d}, J=8.0 \mathrm{~Hz}), 127.2,115.7(\mathrm{~d}, J=21.4 \mathrm{~Hz}), 45.1,30.2,29.4 ;{ }^{19} \mathrm{~F}\left\{{ }^{1} \mathrm{H}\right\}$ NMR $\left(376 \mathrm{MHz}, \mathrm{CDCl}_{3}\right) \delta-115.94 \mathrm{ppm}$. Characterization data matched those obtained from $0.50 \mathrm{mmol}$ scale reactions (see page $\mathrm{S} 35$ ). 


\subsection{Cross-Electrophile Couplings Between Selected Phenol Derivatives}

Scheme S10. Cross-electrophile couplings between selected phenol derivatives

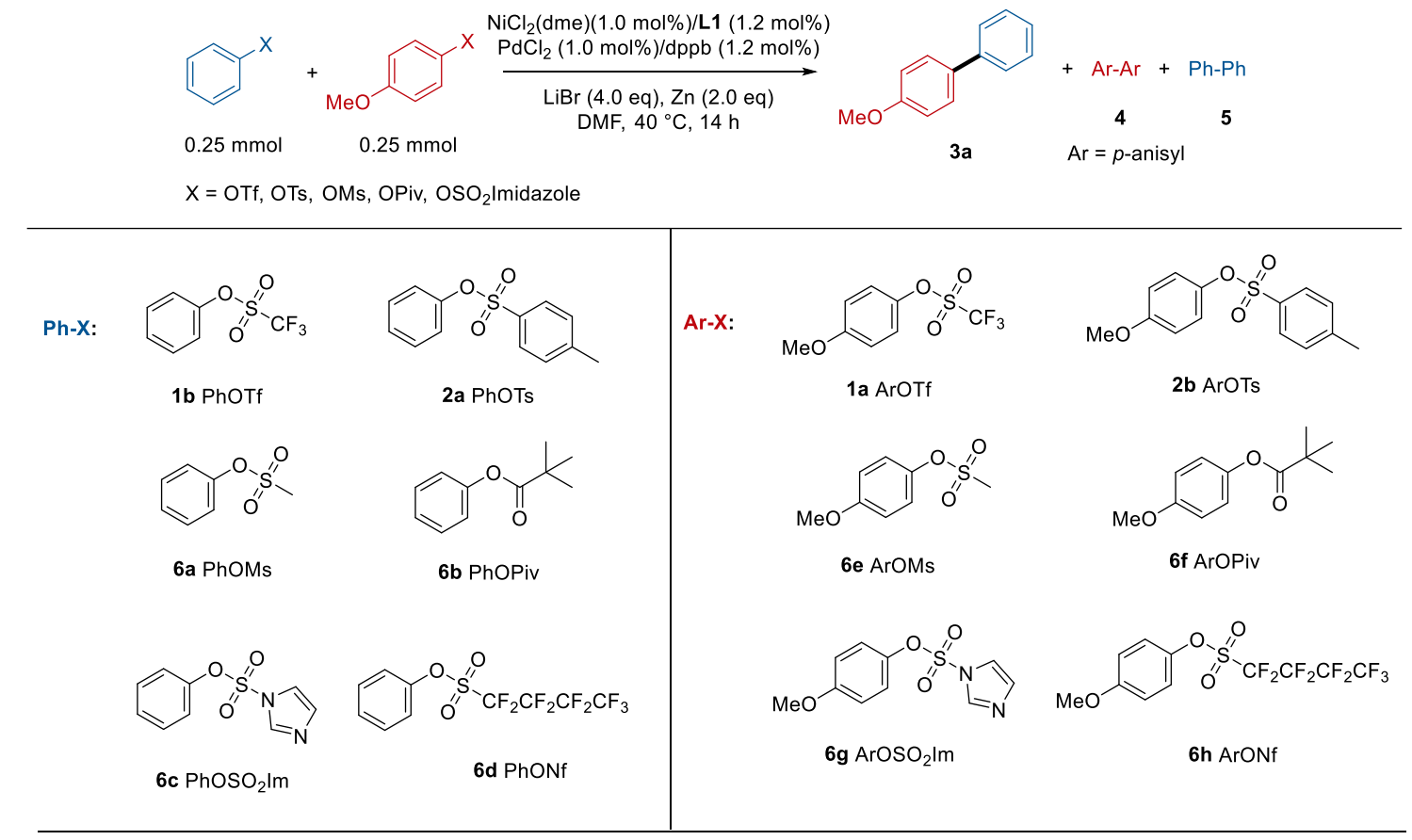

\section{Procedure for cross-electrophile couplings between selected phenol derivatives}

The following procedures were conducted in a nitrogen filled glovebox for convenience. Nickel and palladium catalyst stock solutions were prepared following general procedure for optimization studies (page S4) using $\mathbf{L 1}$ (18.5 mg, $0.060 \mathrm{mmol}$ ) as the amine ligand and dppb (25.6 $\mathrm{mg}, 0.060 \mathrm{mmol}$ ) as the phosphine ligand. Catalytic reactions were setup following general procedure for optimization studies (page S4) using $\mathbf{P h}-\mathbf{X}$ (0.25 mmol, 1.0 equiv) and $\mathbf{A r}-\mathbf{X}$ (0.25 mmol, 1.0 equiv; $\mathrm{Ar}=p$-anisyl) as starting materials, $1.0 \mathrm{~mol} \% \mathrm{NiCl}_{2}(\mathrm{dme}) / \mathbf{L 1}$ and $1.0 \mathrm{~mol} \% \mathrm{PdCl}_{2} / \mathrm{dppb}$ as catalysts, $\mathrm{LiBr}(87 \mathrm{mg}, 1.0 \mathrm{mmol}, 4.0$ equiv) as additive, and $\mathrm{Zn}\left(33 \mathrm{mg}, 0.50 \mathrm{mmol}, 2.0\right.$ equiv) as reductant at $40{ }^{\circ} \mathrm{C}$ (as in Scheme $\left.\mathrm{S} 10\right)$. GC analyses were conducted following general procedure for optimization studies (page S4). 
Table S3. Yields of 3a in cross-electrophile couplings between selected phenol derivatives $\left(\mathrm{Ar}=p\right.$-anisyl). ${ }^{a}$

\begin{tabular}{ccccccc} 
3a $(\%)$ & Ph-OTf & Ph-OTs & Ph-OMs & Ph-OPiv & Ph-ONf & Ph-OSO 2 Im \\
Ar-OTf & 55 & 76 & 19 & 0 & 0 & 47 \\
Ar-OTs & 66 & 36 & 3 & 0 & 0 & 11 \\
Ar-OMs & 13 & 0 & 0 & 0 & 0 & 0 \\
Ar-OPiv & 0 & 0 & 0 & 0 & 0 & 0 \\
Ar-ONf & 0 & 0 & 0 & 0 & 0 & 0 \\
Ar-OSO 2 Im & 63 & 41 & 5 & 0 & 0 & 34 \\
\hline
\end{tabular}

${ }^{a} \mathrm{GC}$ yields using dodecane as an internal standard.

Table S4. Yields of biaryls in cross-electrophile couplings between selected phenol derivatives $(\mathrm{Ar}=p$ anisyl). ${ }^{a}$

\begin{tabular}{ccccccc}
$\mathbf{4}(\%) / \mathbf{3 a}(\%) / \mathbf{5}(\%)$ & $\mathrm{Ph}-\mathrm{OTf}$ & $\mathrm{Ph}-\mathrm{OTs}$ & $\mathrm{Ph}-\mathrm{OMs}$ & $\mathrm{Ph}-\mathrm{OPiv}$ & $\mathrm{Ph}-\mathrm{ONf}$ & $\mathrm{Ph}^{-\mathrm{OSO}_{2} \mathrm{Im}}$ \\
$\mathrm{Ar}-\mathrm{OTf}$ & $22 / 55 / 23$ & $12 / 76 / 13$ & $37 / 19 / 0$ & $0 / 0 / 0$ & $0 / 0 / 0$ & $24 / 47 / 23$ \\
$\mathrm{Ar}-\mathrm{OTs}$ & $2 / 66 / 22$ & $20 / 36 / 25$ & $8 / 3 / 0$ & $0 / 0 / 0$ & $0 / 0 / 0$ & $35 / 11 / 33$ \\
$\mathrm{Ar}-\mathrm{OMs}$ & $0 / 13 / 31$ & $0 / 0 / 22$ & $0 / 0 / 2$ & $0 / 0 / 0$ & $0 / 0 / 0$ & $0 / 0 / 34$ \\
$\mathrm{Ar}-\mathrm{OPiv}$ & $0 / 0 / 17$ & $0 / 0 / 0$ & $0 / 0 / 0$ & $0 / 0 / 0$ & $0 / 0 / 0$ & $0 / 0 / 41$ \\
$\mathrm{Ar}-\mathrm{ONf}$ & $0 / 0 / 0$ & $0 / 0 / 0$ & $0 / 0 / 0$ & $0 / 0 / 0$ & $0 / 0 / 0$ & $0 / 0 / 0$ \\
$\mathrm{Ar}^{-\mathrm{OSO}_{2} \mathrm{Im}}$ & $13 / 63 / 16$ & $25 / 41 / 15$ & $48 / 5 / 1$ & $0 / 0 / 0$ & $0 / 0 / 0$ & $31 / 34 / 33$ \\
\hline
\end{tabular}

${ }^{a} \mathrm{GC}$ yields using dodecane as an internal standard.

Table S5. Yields of recovered phenol derivatives after the reactions $(\mathrm{Ar}=p$-anisyl $){ }^{a}$

\begin{tabular}{ccccccc} 
Ar-X $(\%) / P h-X(\%)$ & Ph-OTf & Ph-OTs & Ph-OMs & Ph-OPiv & Ph-ONf & Ph-OSO $I_{2}$ Im \\
Ar-OTf & $0 / 0$ & $0 / 0$ & $0 / 62$ & $42 / 86$ & $99 / 20$ & $0 / 0$ \\
Ar-OTs & $0 / 0$ & $0 / 0$ & $34 / 58$ & $94 / 88$ & $91 / 17$ & $0 / 0$ \\
Ar-OMs & $65 / 0$ & $82 / 25$ & $85 / 87$ & $92 / 90$ & $89 / 18$ & $67 / 0$ \\
Ar-OPiv & $84 / 0$ & $93 / 70$ & $93 / 92$ & $87 / 89$ & $93 / 22$ & $83 / 0$ \\
Ar-ONf & $15 / 91$ & $17 / 95$ & $14 / 93$ & $24 / 88$ & $42 / 43$ & $52 / \mathrm{X}^{b}$ \\
Ar-OSO 2 Im & $0 / 0$ & $0 / 0$ & $0 / 54$ & $\mathrm{X}^{b} / 92$ & $\mathrm{X}^{b} / 33$ & $0 / 0$ \\
\hline
\end{tabular}

${ }^{a} \mathrm{GC}$ yields using dodecane as an internal standard. ${ }^{b}$ The yields (shown as $\mathrm{X}$ in entries 5 and 6 ) of $\mathrm{Ph}-\mathrm{OSO}_{2} \mathrm{Im}$ (6c) or $\mathrm{Ar}_{-} \mathrm{OSO}_{2} \mathrm{Im}(\mathbf{6 g})$ after the reactions cannot be determined by GC due to their decomposition at high temperatures. However, no GC signals from the decompositions of them could be observed if they were fully consumed after reactions. This was used as the standard to determine if they were fully consumed. 


\subsection{Cross-Electrophile Couplings of $p$-Anisyl Triflate (1a) with Selected Aryl Arenesulfonate Esters}

Scheme S11. Cross-electrophile couplings of 1a with selected aryl arenesulfonate esters

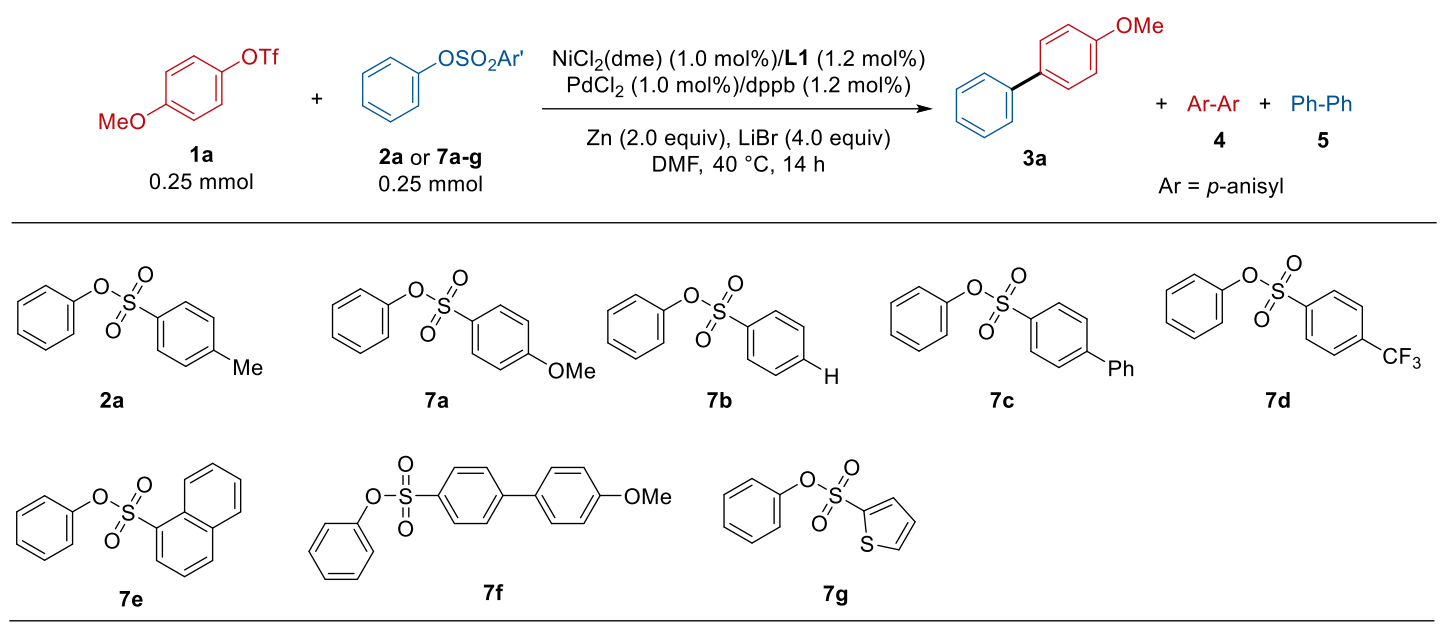

\section{Procedure for cross-electrophile couplings of 1a with selected aryl arenesulfonate esters}

The following procedures were conducted in a $\mathrm{N}_{2}$ filled glovebox for convenience. Nickel and palladium catalyst stock solutions were prepared following general procedure for optimization studies (page S4) using $\mathbf{L 1}$ (18.5 mg, $0.060 \mathrm{mmol}$ ) as the amine ligand and dppb (25.6 mg, $0.060 \mathrm{mmol}$ ) as the phosphine ligand. Catalytic reactions were setup following general procedure for optimization studies (page S4) using 1a ( $0.25 \mathrm{mmol}, 1.0$ equiv) and $\mathbf{2 a}$ or $\mathbf{7 a - g}(0.25 \mathrm{mmol}, 1.0$ equiv) as starting materials, 1.0 $\mathrm{mol} \% \mathrm{NiCl}_{2}(\mathrm{dme}) / \mathbf{L 1}$ and $1.0 \mathrm{~mol} \% \mathrm{PdCl}_{2} / \mathrm{dppb}$ as catalysts, $\mathrm{LiBr}(87 \mathrm{mg}, 1.0 \mathrm{mmol}, 4.0$ equiv) as additive, and $\mathrm{Zn}$ (33 mg, $0.50 \mathrm{mmol}, 2.0$ equiv) as reductant at $40{ }^{\circ} \mathrm{C}$ (as in Scheme S11). GC analyses were conducted following general procedure for optimization studies (page S4).

Table S6. Yields of 3a, 4 and $\mathbf{5}$ in cross-electrophile couplings of 1a with selected aryl arenesulfonate esters $^{a}$

\begin{tabular}{ccccc} 
entry & Ar' & $\mathbf{3 a}(\%)$ & $\mathbf{4}(\%)$ & $\mathbf{5}(\%)$ \\
\hline 1 & 4-methylphenyl, 2a & 76 & 12 & 13 \\
2 & p-anisyl, 7a & 80 & 11 & 10 \\
3 & phenyl, 7b & 30 & 13 & 18 \\
4 & 1,1'-biphenyl-4-yl, 7c & 42 & 19 & 6 \\
5 & 4-trifluoromethylphenyl, 7d & 5 & 37 & 10 \\
6 & 1-naphthyl, 7e & 30 & 22 & 3 \\
7 & 4'-methoxy-1,1'-biphenyl-4-yl, 7f & 74 & 10 & 6 \\
8 & 2-thiophenyl, 7g & 0 & 0 & 0 \\
\hline
\end{tabular}

${ }^{a}$ GC yields using dodecane as an internal standard. 
4.4 Hammett Constants of Substituents on Selected Aryl Triflates and Aryl Tosylates and Their Influence on the Yield of Cross-Coupled Product

Scheme S12. Cross-electrophile coupling of phenyl tosylate with 3-fluorophenyl triflate ${ }^{a, b, c}$

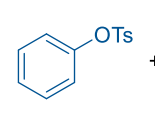

$0.25 \mathrm{mmol}$

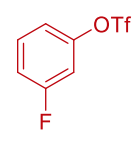

$0.25 \mathrm{mmol}$

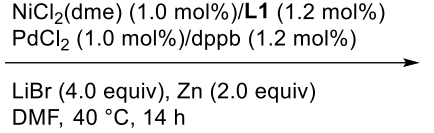

DMF, $40{ }^{\circ} \mathrm{C}, 14 \mathrm{~h}$

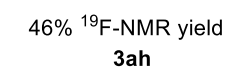

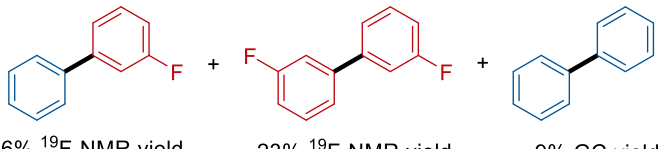

$23 \%{ }^{19} \mathrm{~F}-\mathrm{NMR}$ yield

$9 \%$ GC yield

${ }^{a}$ 4-Trifluoromethyl-1,1'-biphenyl was used as an internal standard for ${ }^{19} \mathrm{~F}-\mathrm{NMR} .{ }^{b}$ Dodecane was used as an internal standard for GC analysis. ${ }^{c}$ Reaction was set up according to general procedure for optimization studies (page S4).

Table S7. The relationship of Hammett constants of substituents on aryl triflates and yields of products

$\begin{array}{cccc}\text { product } & \text { aryl substitution } & \sigma^{a} & \text { yield }(\%)^{b} \\ \text { 3a } & p \text {-OMe } & \sigma_{p}=-0.27 & 72 \\ \text { 3b } & p \text {-H } & \sigma_{p}=0 & 62 \\ \text { 3e } & m \text {-OMe } & \sigma_{m}=0.12 & 57 \\ 3 \mathbf{i} & m-\mathrm{NMe} 2 & \sigma_{m}=-0.16 & 67 \\ \text { 3k } & p \text {-OMe } & \sigma_{p}=-0.27 & 57 \\ \text { 3r } & p-\mathrm{OMe} & \sigma_{p}=-0.27 & 82 \\ \text { 3u } & p-\mathrm{OMe} & \sigma_{p}=-0.27 & 57 \\ \text { 3ac } & p-\mathrm{OMe} & \sigma_{p}=-0.27 & 64 \\ \text { 3ah } & m-\mathrm{F} & \sigma_{m}=0.34 & 46^{c}\end{array}$

${ }^{a}$ Data source: Chem. Rev. 1991, 91, $165-195 .{ }^{b}$ Isolated yields on $0.50 \mathrm{mmol}$ scale. ${ }^{c 19} \mathrm{~F}$ NMR yield.

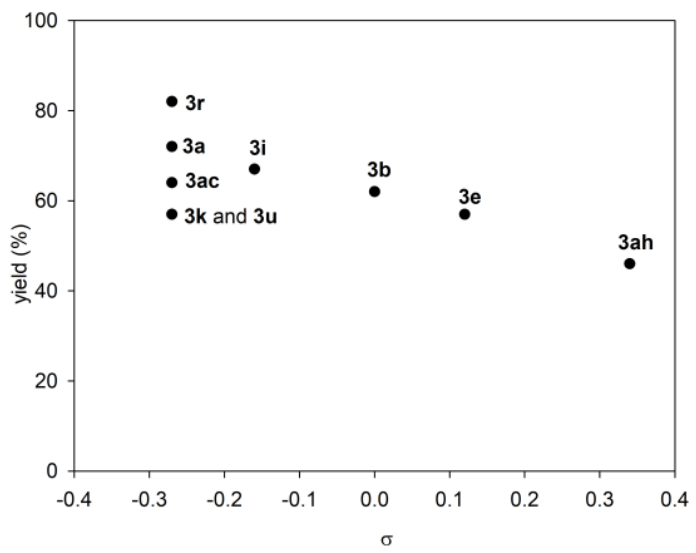

Figure S4. The relationship of Hammett constants of substituents on aryl triflates and yields of products. 
Table S8. The relationship of Hammett constants of aryl substituents on tosylates and yields of products

\begin{tabular}{cccc} 
product & aryl substitution & $\sigma^{a}$ & yield $(\%)^{b}$ \\
3a & $p-\mathrm{H}$ & $\sigma_{p}=0$ & 72 \\
3b & $p-\mathrm{OMe}$ & $\sigma_{p}=-0.27$ & 62 \\
3c & $p-\mathrm{H}$ & $\sigma_{p}=0$ & 70 \\
3e & $m-\mathrm{COMe}$ & $\sigma_{m}=0.38$ & 57 \\
3f & $p-\mathrm{COOMe}$ & $\sigma_{p}=0.45$ & 51 \\
3h & $p-\mathrm{CF}_{3}$ & $\sigma_{p}=0.54$ & 60 \\
$3 \mathbf{3 i}$ & $p-\mathrm{OCF}_{3}$ & $\sigma_{p}=0.35$ & 67 \\
31 & $m-\mathrm{Me}$ & $\sigma_{m}=-0.07$ & 75 \\
\hline
\end{tabular}

${ }^{a}$ Data source: Chem. Rev. 1991, 91, 165-195. ${ }^{b}$ Isolated yields on $0.50 \mathrm{mmol}$ scale.

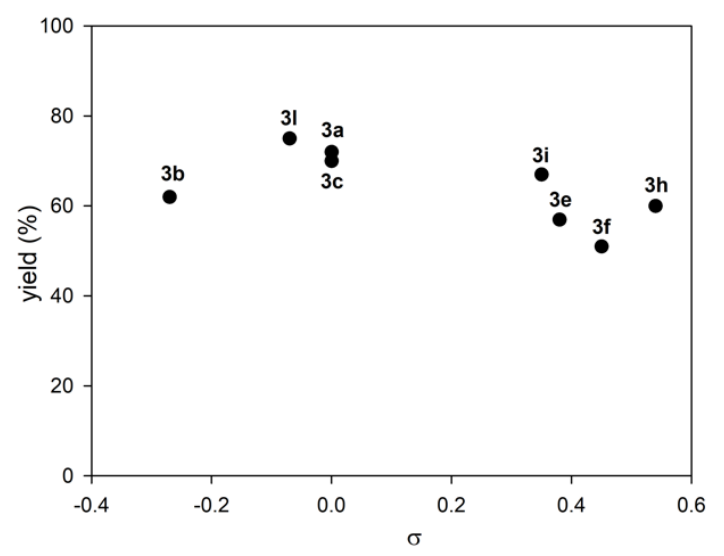

Figure S5. The relationship of Hammett constants of aryl substituents on tosylates and yields of products.

Table S9. The relationship of the difference in Hammett constants $\left(\Delta \sigma=\sigma_{\mathrm{OTs}}-\sigma_{\mathrm{OTf}}\right)$ of substituents on aryl triflates and tosylates and yields of products

\begin{tabular}{ccccc} 
product & $\begin{array}{c}\text { substitution on aryl } \\
\text { tosylate }\end{array}$ & $\begin{array}{c}\text { substitution on aryl } \\
\text { triflate }\end{array}$ & \multicolumn{1}{c}{ yield $(\%)^{b}$} \\
3a & $p-\mathrm{H}$ & $p$-OMe & 0.27 & 72 \\
3b & $p$-OMe & $p$-H & -0.27 & 62 \\
3e & $m-\mathrm{COMe}$ & $m$-OMe & 0.26 & 57 \\
3i & $p-\mathrm{OCF}_{3}$ & $m$-NMe & 0.51 & 67 \\
3k & $p-\mathrm{Cl}$ & $p$-OMe & 0.50 & 57 \\
3ah & $p-\mathrm{H}$ & $m-\mathrm{F}$ & -0.34 & $46^{c}$ \\
\hline
\end{tabular}

${ }^{a}$ Data source: Chem. Rev. 1991, 91, 165-195. ${ }^{b}$ Isolated yields on $0.50 \mathrm{mmol}$ scale. ${ }^{c 19} \mathrm{~F}$ NMR yield. 


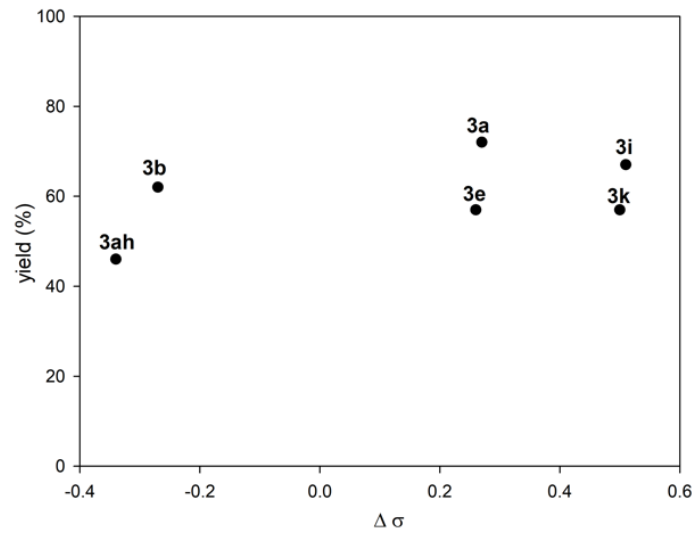

Figure S6. The relationship of the difference in Hammett constants $\left(\Delta \sigma=\sigma_{\mathrm{OTs}}-\sigma_{\mathrm{OTf}}\right)$ of substituents on aryl triflates and tosylates and yields of products

\subsection{Aryl Sulfonate Esters That Are Not Suitable for Nickel and Palladium Multimetallic Conditions}

In the substrate scope studies, we found that some aryl sulfonate esters were not successful. Reactions of these aryl triflates with phenyl tosylate (2a) and reactions of these aryl tosylates with $p$-anisyl triflate (1a) were attempted under standard reaction conditions on $0.25 \mathrm{mmol} \mathrm{scale}$, but no product or only trace amount of product was observed by GC or SFC-MS analysis.

Scheme S13. Aryl sulfonate esters that failed to couple under standard conditions
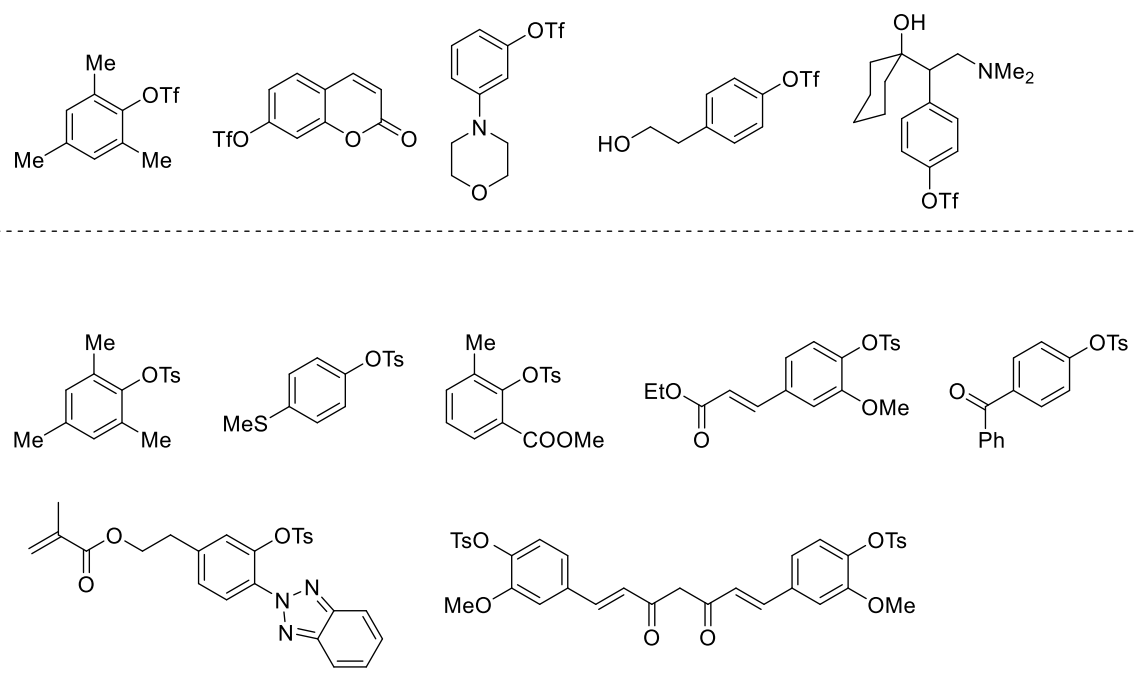


\section{Mechanistic Studies}

\subsection{Background Reactions of Zn with $p$-Anisyl Triflate (1a) and Phenyl Tosylate (2a)}

Scheme S14. Control reactions of 19 and $2 \mathrm{a}$ with zinc, with and without $\mathrm{LiBr}^{a}$

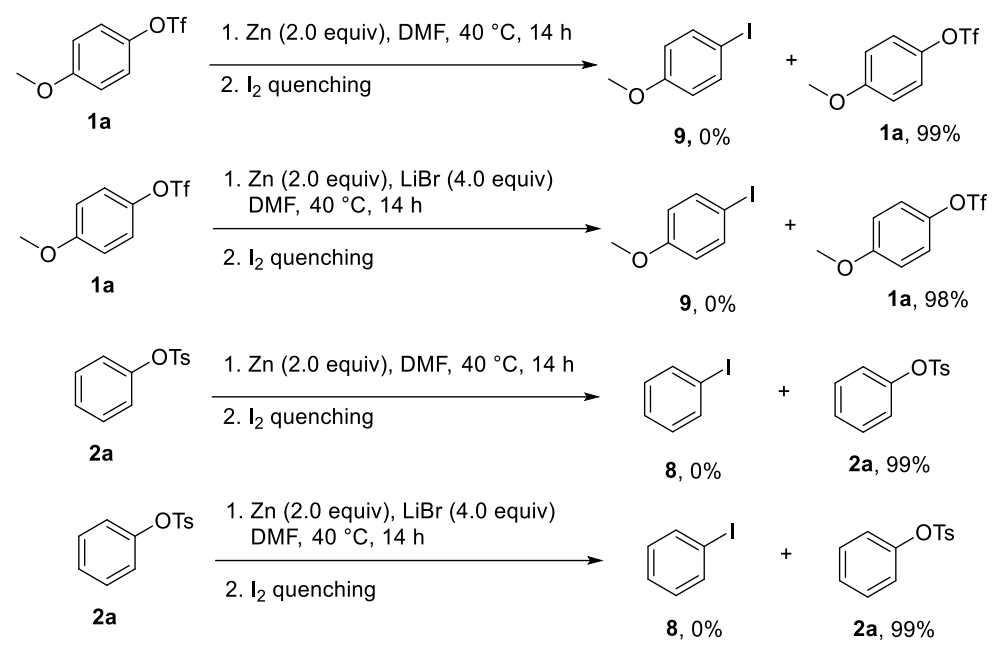

${ }^{a} \mathrm{GC}$ yields using 1,3,5-trimethoxybenzene as an internal standard.

\section{Preparation of $\mathbf{I}_{\mathbf{2}}$ and $\mathbf{L i C l}$ quenching solution ${ }^{40,55}$}

In a $\mathrm{N}_{2}$ filled glovebox, $\mathrm{LiCl}(127 \mathrm{mg}, 3.00 \mathrm{mmol})$ and THF $(6.0 \mathrm{~mL})$ were added to an oven-dried scintillation vial equipped with a PTFE-coated stir-bar. The solution was stirred at rt overnight until all solids were dissolved, resulting in a $0.5 \mathrm{M}$ solution of $\mathrm{LiCl}$. In a separate scintillation vial was weighed $\mathrm{I}_{2}$ (254 mg, $1.00 \mathrm{mmol}$ ), and $4 \mathrm{~mL}$ of the $0.5 \mathrm{M}$ solution of $\mathrm{LiCl}$ was added. The resulting brown solution was stirred at $\mathrm{rt}$ for 1 hour before being used.

\section{Procedure for reactions of $\mathrm{Zn}$ with $1 \mathrm{a}$ and $2 \mathrm{a}$ in $\mathrm{DMF}$ at $40^{\circ} \mathrm{C}$}

In a $\mathrm{N}_{2}$ filled glovebox, an oven-dried 1-dram vial fitted with a PTFE-coated stir-bar was charged sequentially with $1 \mathbf{a}(64 \mathrm{mg}, 0.25 \mathrm{mmol}, 1.0$ equiv) or $\mathbf{1 b}$ (62 mg, $0.25 \mathrm{mmol}, 1.0$ equiv), Zn (33 mg, 0.50 mmol, 2.0 equiv), $\mathrm{LiBr}$ (0 or $87 \mathrm{mg}, 1.0 \mathrm{mmol}, 4.0$ equiv), and 1,3,5-trimethoxybenzene (15-20 $\mathrm{mg}$ ) as an internal standard. DMF $(1.0 \mathrm{~mL})$ was added before the vial was capped with a screw cap fitted with a PTFEfaced silicone septum. The reaction mixture was stirred $(1250 \mathrm{rpm})$ in a reaction block set to $40{ }^{\circ} \mathrm{C}$ in the glovebox for $14 \mathrm{~h}$. A $100 \mu \mathrm{L}$ aliquot of the reaction mixture was transferred into another 1-dram vial equipped with a PTFE-coated stir-bar. Iodine- $\mathrm{LiCl}$ solution $(200 \mu \mathrm{L}, 0.05 \mathrm{mmol})$ was added using a pipette before the vial was capped with a screw cap fitted with a PTFE-faced silicone septum. The quenched mixture was stirred at $\mathrm{rt}$ in the glovebox for $20 \mathrm{~min}$ before the vial was removed from the glovebox. The mixture was diluted with ethyl acetate $(2 \mathrm{~mL})$ and filtered through a 2-cm silica gel plug in a Pasteur pipette. The resulting filtrate was collected in a GC vial. The sample was analyzed by GC and the yields were 
determined based on the peak area of the analyte compared to 1,3,5-trimethoxybenzene as an internal standard.

Results: Neither of the starting materials were consumed and no aryl iodides were formed, confirming that zinc does not react with aryl tosylates or triflates to form arylzinc reagents without a catalyst.

\subsection{Background Reactions of LiBr with $p$-Anisyl Triflate (1a) and Phenyl Tosylate (2a)}

Scheme S15. Reactions of LiBr with 1 a and $2 \mathbf{a}$ in DMF at $40{ }^{\circ} \mathrm{C}$

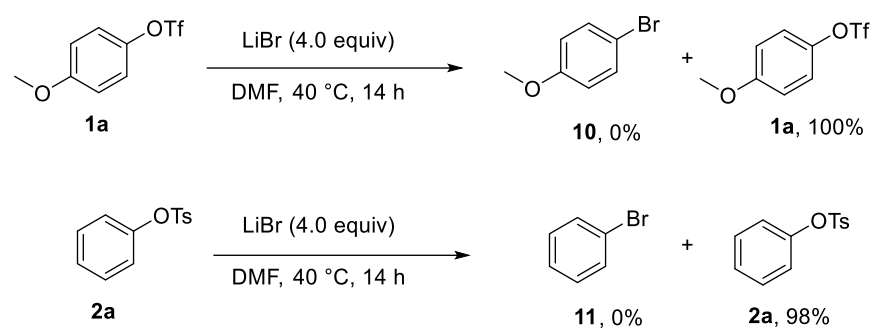

${ }^{a} \mathrm{GC}$ yields using 1,3,5-trimethoxybenzene as an internal standard.

\section{Procedure for reactions of $\mathrm{LiBr}$ with $1 \mathrm{a}$ and $2 \mathrm{a}$ in $\mathrm{DMF}$ at $40{ }^{\circ} \mathrm{C}$}

In a $\mathrm{N}_{2}$ filled glovebox, an oven-dried 1-dram vial equipped with a PTFE-coated stir-bar was charged sequentially with $\mathbf{1 a}(64 \mathrm{mg}, 0.25 \mathrm{mmol}, 1.0$ equiv) or $\mathbf{1 b}(62 \mathrm{mg}, 0.25 \mathrm{mmol}, 1.0$ equiv), $\mathrm{LiBr}(87$ $\mathrm{mg}, 1.0 \mathrm{mmol}, 4.0$ equiv), and 1,3,5-trimethoxybenzene (15-20 mg) as an internal standard. DMF (1.0 mL) was added before the vial was capped with a screw cap fitted with a PTFE-faced silicone septum. The vial was removed from the glovebox, and the mixture was stirred $(1250 \mathrm{rpm})$ in a reaction block set to $40{ }^{\circ} \mathrm{C}$ for $14 \mathrm{~h}$. A $100 \mu \mathrm{L}$ aliquot of the reaction mixture was removed, diluted with ethyl acetate $(2 \mathrm{~mL})$, and filtered through a $2-\mathrm{cm}$ silica gel plug in a Pasteur pipette. The resulting filtrate was collected in a GC vial. The sample was analyzed by GC and the yields were determined based on the peak area of the analyte compared to 1,3,5-trimethoxybenzene as an internal standard.

Results: No conversion of the aryl sulfonate esters to aryl bromides was observed. 
5.3 Iodine Quenching Experiments for Reactions of $p$-Anisyl Triflate (1a) with Phenyl Tosylate (2a) in the Presence of Nickel and/or Palladium Catalysts

General procedure for iodine quenching experiments

The following procedures were conducted in a $\mathrm{N}_{2}$ filled glovebox for convenience. Nickel and palladium catalyst stock solutions were prepared following general procedure for optimization studies (page S4) using $\mathbf{L 1}$ (18.5 $\mathrm{mg}, 0.060 \mathrm{mmol}$ ) as the amine ligand and dppb (25.6 $\mathrm{mg}, 0.060 \mathrm{mmol})$ as the phosphine ligand. Reactions were setup following general procedure for optimization studies (page S4) using 1a (64 mg, $0.25 \mathrm{mmol}, 1.0$ equiv) and $\mathbf{1 b}(62 \mathrm{mg}, 0.25 \mathrm{mmol}, 1.0$ equiv) as starting materials, 0 or $1.0 \mathrm{~mol} \% \mathrm{NiCl}_{2}(\mathrm{dme}) / \mathbf{L 1}$ and $0,1.0$, or $5.0 \mathrm{~mol} \% \mathrm{PdCl}_{2} / \mathrm{dppb}$ as catalysts, $\mathrm{LiBr}(87 \mathrm{mg}, 1.0 \mathrm{mmol}, 4.0$ equiv) as additive, and $\mathrm{Zn}\left(33 \mathrm{mg}, 0.50 \mathrm{mmol}, 2.0\right.$ equiv) as reductant at $40{ }^{\circ} \mathrm{C}$.

\section{Iodine quenching and GC analysis}

At $0,20,40,60,120,180,300$ and $420 \mathrm{~min}$, a $25 \mu \mathrm{L}$ aliquot of the reaction mixture was removed and transferred into another 1-dram vial fitted with a PTFE-coated stir-bar. Iodine-LiCl solution (200 $\mu \mathrm{L}$, $0.05 \mathrm{mmol}$ ) was added using a pipette before the vial was capped with a screw cap fitted with a PTFE-faced silicone septum. The quenched mixture was stirred at $\mathrm{rt}$ in the glovebox for $20 \mathrm{~min}$, and the vial was removed from the glovebox. The quenched mixture was diluted with ethyl acetate $(2 \mathrm{~mL})$ and filtered through a $2-\mathrm{cm}$ silica gel plug in a Pasteur pipette. The resulting filtrate was collected in a GC vial. The sample was analyzed by GC and the yields were determined based on the peak area of the analyte compared to 1,3,5-trimethoxybenzene as an internal standard. Concentrations lower than $2.5 \times 10^{-4} \mathrm{M}$ (lower limit of detection) were recorded as 0 . 
5.3.1 Iodine Quenching Experiments for the Reaction of $p$-Anisyl Triflate (1a) with Phenyl Tosylate (2a) under Standard Conditions (Condition A)

Table S10. Concentrations of 1a, 2a, 3a, 4 and 5 in Condition $\mathrm{A}^{a}$

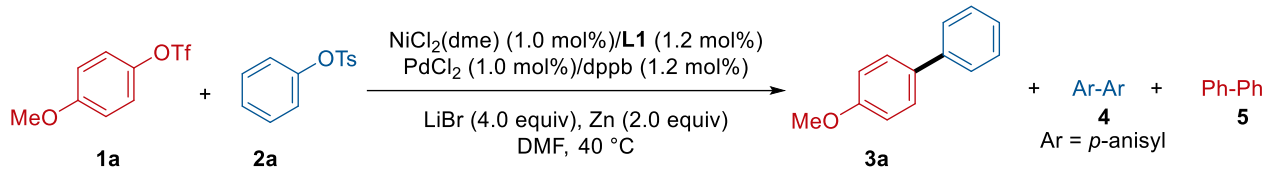

$\begin{array}{cccccc}\text { time (min) } & {[\mathbf{1 a}](\mathbf{M})} & {[\mathbf{2 a}](\mathbf{M})} & {[\mathbf{3 a}](\mathbf{M})} & {[\mathbf{4}](\mathbf{M})} & {[\mathbf{5}](\mathbf{M})} \\ 0 & 0.250 & 0.250 & 0 & 0 & 0 \\ 20 & 0.245 & 0.245 & 0.002 & 0 & 0 \\ 40 & 0.242 & 0.238 & 0.005 & 0.002 & 0.002 \\ 60 & 0.232 & 0.225 & 0.010 & 0.003 & 0.005 \\ 120 & 0.190 & 0.162 & 0.050 & 0.010 & 0.015 \\ 180 & 0.108 & 0.095 & 0.105 & 0.020 & 0.020 \\ 300 & 0.015 & 0.020 & 0.178 & 0.030 & 0.022 \\ 420 & 0 & 0 & 0.185 & 0.032 & 0.025\end{array}$

${ }^{a}$ Concentrations determined by GC analysis using 1,3,5-trimethoxybenzene as an internal standard.

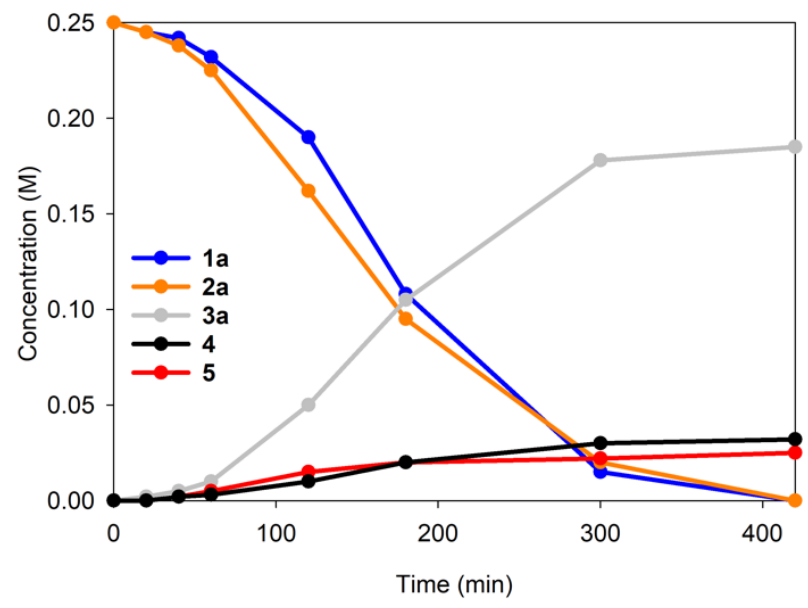

Figure S7. Plot of the concentrations of 1a (Ar-OTf), 2a (Ph-OTs), 3a (Ph-Ar), $4(\mathrm{Ar}-\mathrm{Ar})$ and $\mathbf{5}(\mathrm{Ph}-\mathrm{Ph})$ in Condition A over time. $\mathrm{Ar}=p$-anisyl. 
Table S11. Concentrations of $\mathbf{8}$ and $\mathbf{9}$ and the [aryl iodide]/[Ni] ratios from iodine quenching experiments in Condition $\mathrm{A}^{a}$

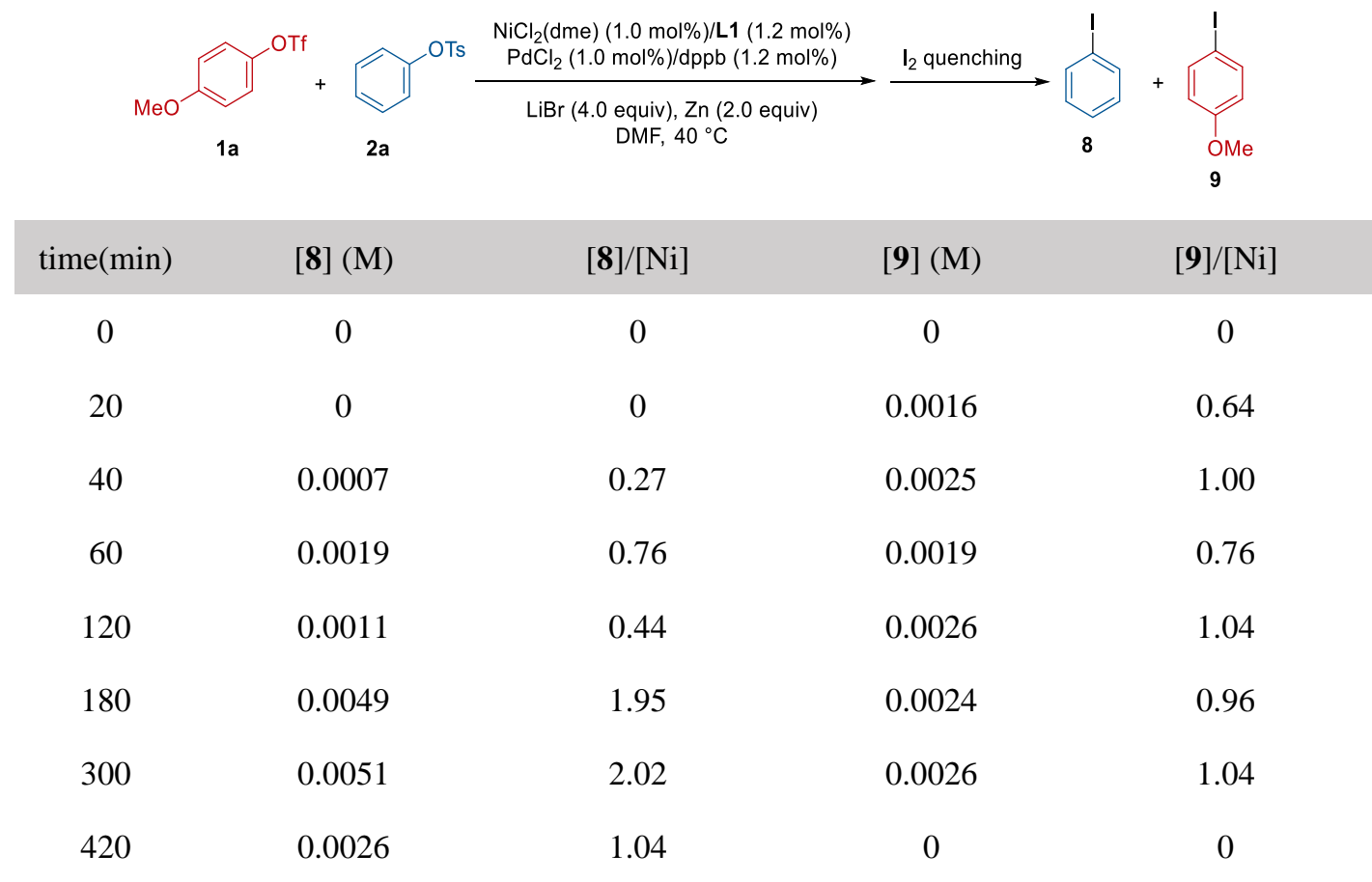

${ }^{a}$ Concentrations determined by GC analysis using 1,3,5-trimethoxybenzene as an internal standard.

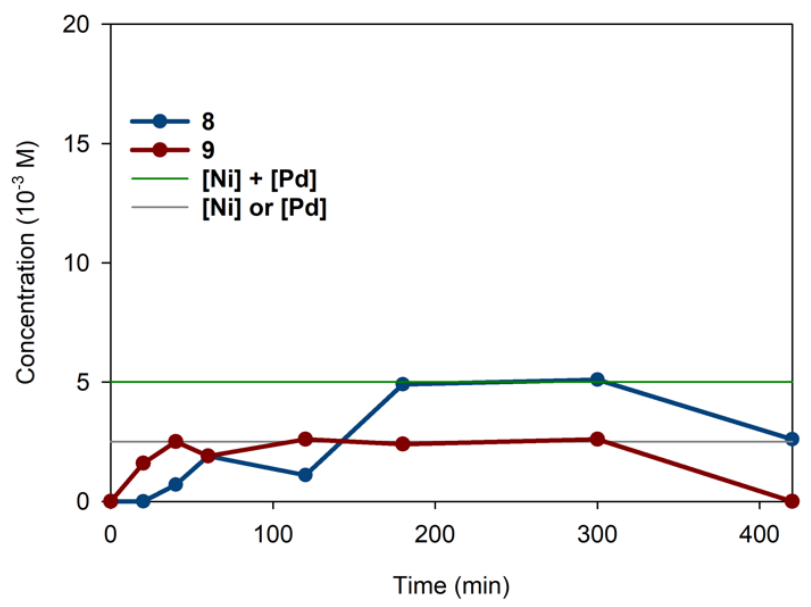

Figure S8. Plot of concentrations of iodobenzene (8) and $p$-iodoanisole (9) from iodine-quenched aliquots of Condition A over time. 
5.3.2 Iodine Quenching Experiments for the Reaction of $p$-Anisyl Triflate (1a) with Phenyl Tosylate (2a) under Ni-Only Conditions (Condition B)

Table S12. Concentrations of 1a, 2a, 3a, 4 and 5 in Condition $\mathrm{B}^{a}$
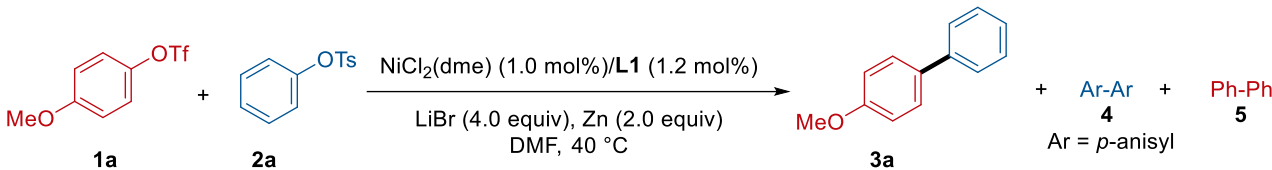

$\begin{array}{cccccc}\text { time }(\min ) & {[\mathbf{1 a}](\mathbf{M})} & {[\mathbf{2 a}](\mathbf{M})} & {[\mathbf{3 a}](\mathbf{M})} & {[\mathbf{4}](\mathbf{M})} & {[\mathbf{5}](\mathbf{M})} \\ 0 & 0.250 & 0.250 & 0 & 0 & 0 \\ 20 & 0.247 & 0.250 & 0 & 0 & 0 \\ 40 & 0.241 & 0.248 & 0.005 & 0 & 0.004 \\ 60 & 0.236 & 0.234 & 0.008 & 0.001 & 0.006 \\ 120 & 0.188 & 0.174 & 0.040 & 0.006 & 0.015 \\ 180 & 0.120 & 0.103 & 0.088 & 0.015 & 0.024 \\ 300 & 0.031 & 0.069 & 0.132 & 0.029 & 0.028 \\ 420 & 0 & 0 & 0.145 & 0.032 & 0.031\end{array}$

${ }^{a}$ Concentrations determined by GC analysis using 1,3,5-trimethoxybenzene as an internal standard.

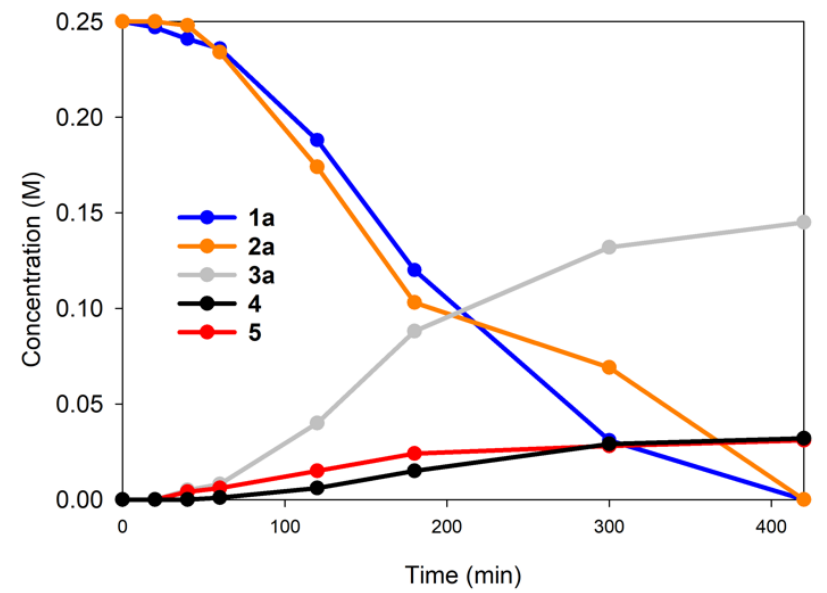

Figure S9. Plot of the concentrations of $\mathbf{1 a}(\mathrm{Ar}-\mathrm{OTf}), \mathbf{2 a}(\mathrm{Ph}-\mathrm{OTs}), \mathbf{3 a}(\mathrm{Ph}-\mathrm{Ar}), \mathbf{4}(\mathrm{Ar}-\mathrm{Ar})$ and $\mathbf{5}(\mathrm{Ph}-\mathrm{Ph})$ in Condition B over time. Ar $=p$-anisyl. 
Table S13. Concentrations of $\mathbf{8}$ and $\mathbf{9}$ and the [aryl iodide]/[Ni] ratios from iodine quenching experiments in Condition $\mathrm{B}^{a}$

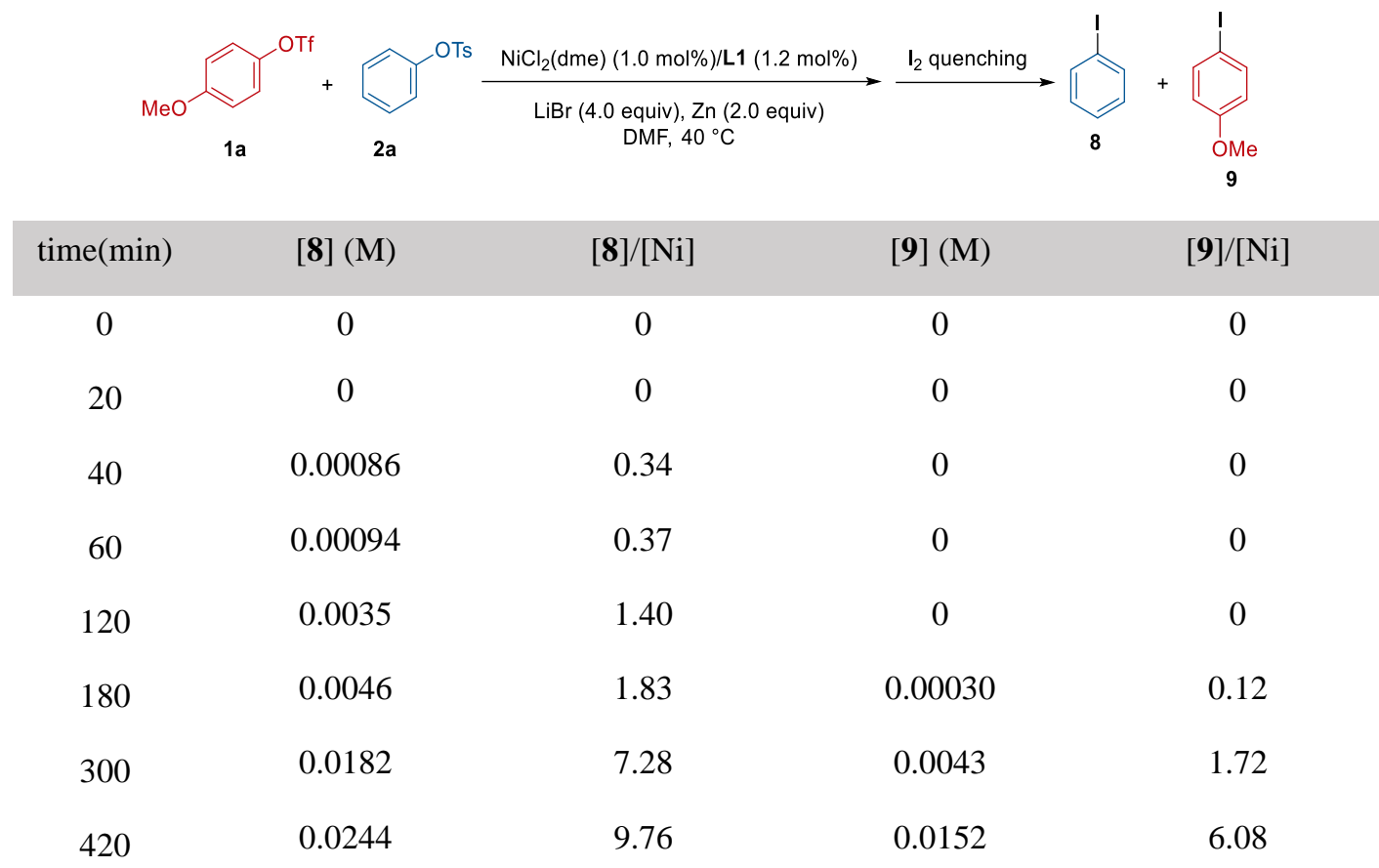

${ }^{a}$ Concentrations determined by GC analysis using 1,3,5-trimethoxybenzene as an internal standard.

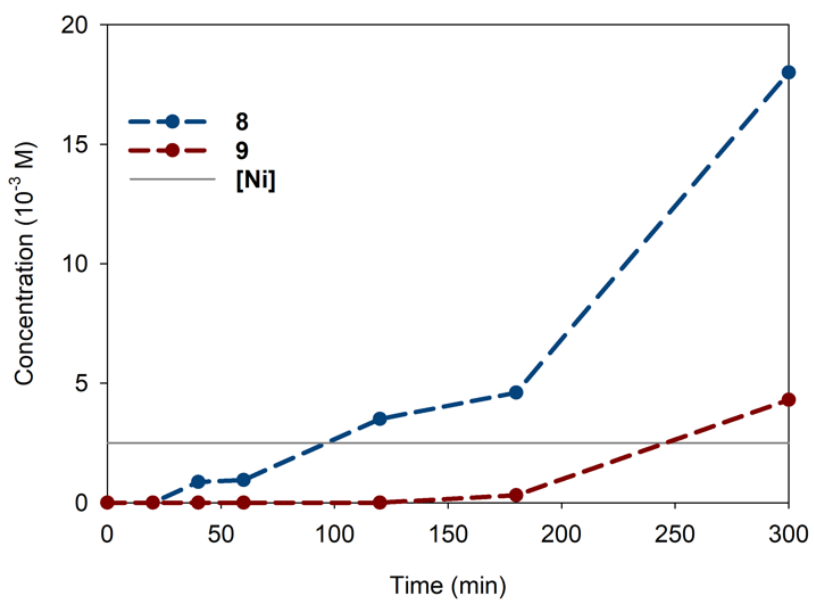

Figure S10. Plot of concentrations of iodobenzene (8) and $p$-iodoanisole (9) from iodine-quenched aliquots of Condition B over time. 


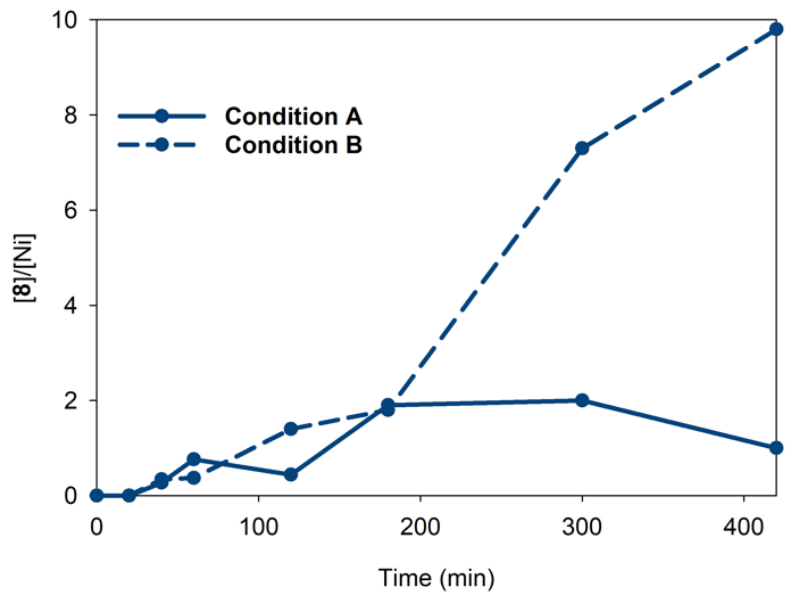

Figure S11. Plot of [8]/[Ni] ratios from iodine-quenched aliquots of Condition A and B over time.

5.3.3 Iodine Quenching Experiments for the Reaction of $p$-Anisyl Triflate (1a) with Phenyl Tosylate (2a) under Pd-Only Conditions (Condition C)

Table S14. Concentrations of 1a, 2a, 3a (Ph-Ar), $4(\mathrm{Ar}-\mathrm{Ar})$ and $5(\mathrm{Ph}-\mathrm{Ph})$ in Condition $\mathrm{C}(\mathrm{Ar}=p \text {-anisyl })^{a}$

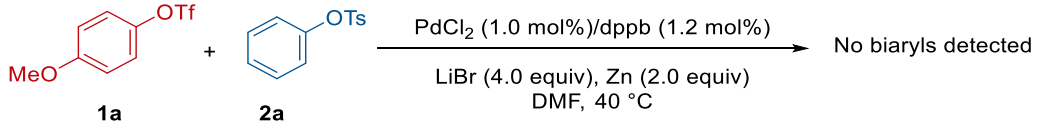

\begin{tabular}{cccccc} 
time $(\min )$ & {$[\mathbf{1 a}](\mathbf{M})$} & {$[\mathbf{2} \mathbf{a}](\mathbf{M})$} & {$[\mathbf{3 a}](\mathbf{M})$} & {$[\mathbf{4}](\mathbf{M})$} & {$[\mathbf{5}](\mathbf{M})$} \\
0 & 0.250 & 0.256 & 0 & 0 & 0 \\
20 & 0.250 & 0.256 & 0 & 0 & 0 \\
40 & 0.252 & 0.247 & 0 & 0 & 0 \\
60 & 0.249 & 0.250 & 0 & 0 & 0 \\
120 & 0.242 & 0.250 & 0 & 0 & 0 \\
180 & 0.244 & 0.249 & 0 & 0 & 0 \\
300 & 0.240 & 0.252 & 0 & 0 & 0 \\
420 & 0.240 & 0.246 & 0 & 0 & 0 \\
\hline
\end{tabular}

${ }^{a}$ Concentrations determined by GC analysis using 1,3,5-trimethoxybenzene as an internal standard. 
Table S15. Concentrations of $\mathbf{8}$ and $\mathbf{9}$ and the [aryl iodide]/[Pd] ratios from iodine quenching experiments of Condition $\mathrm{C}^{a}$

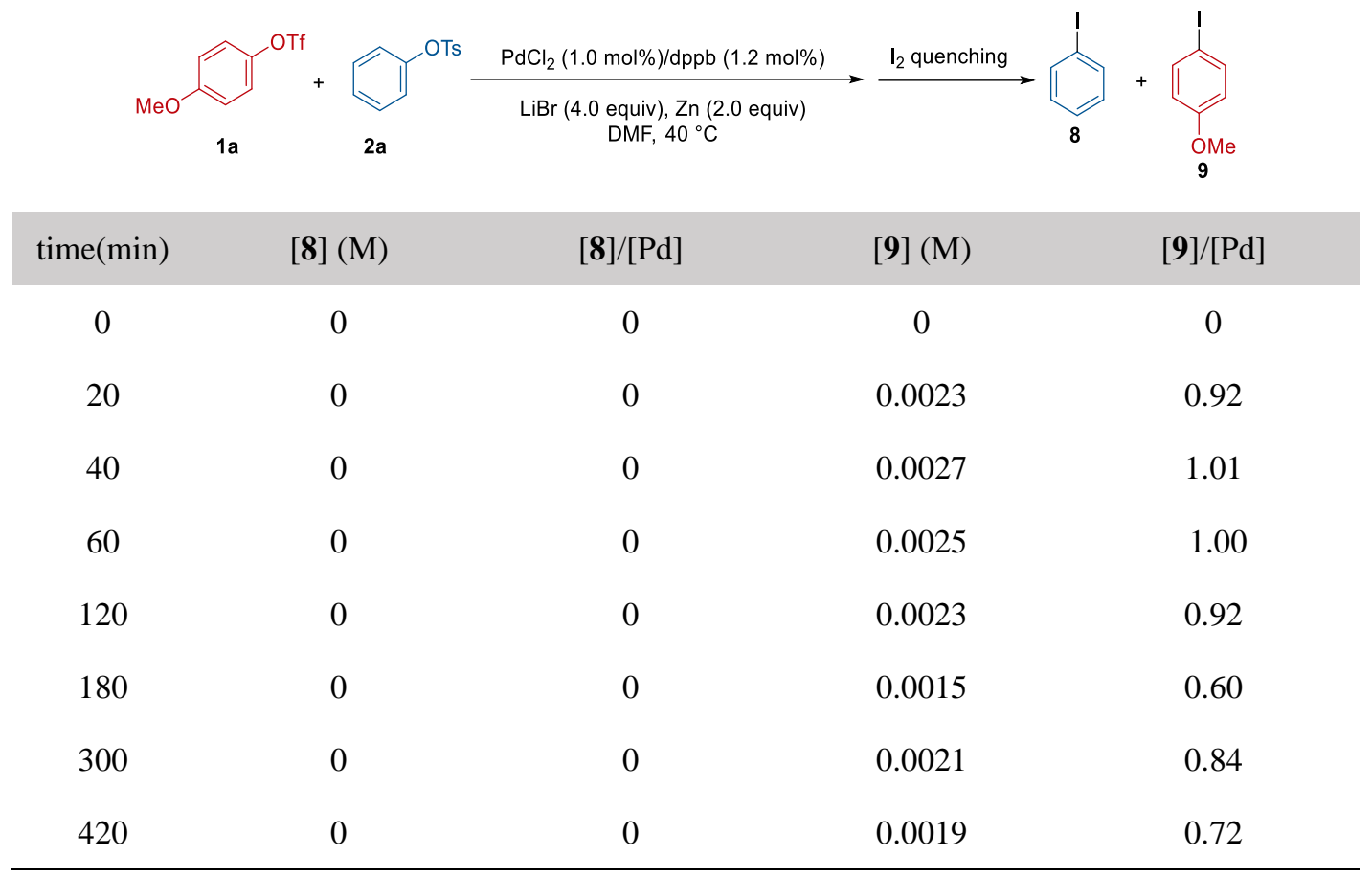

${ }^{a}$ Concentrations determined by GC analysis using 1,3,5-trimethoxybenzene as an internal standard.

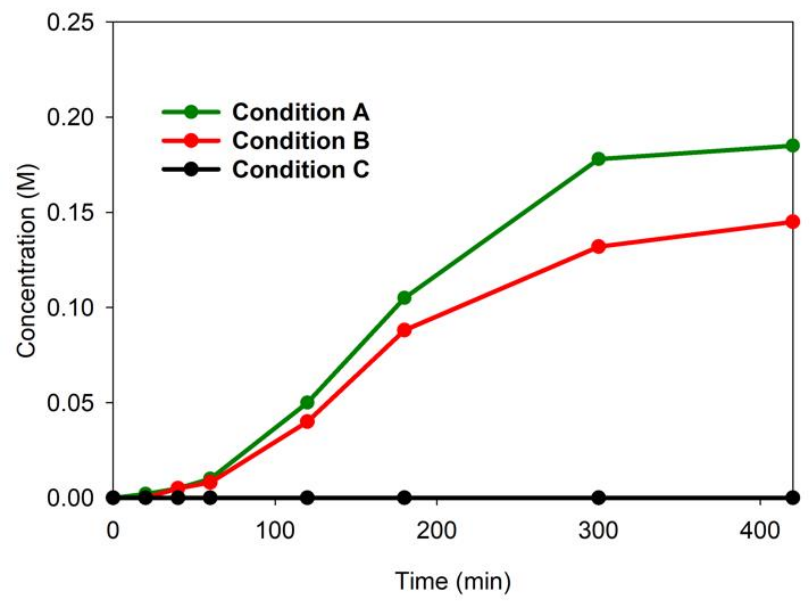

Figure 12. Plot of concentration of 3a in Condition A $(\mathrm{Ni}+\mathrm{Pd})$, Condition B (Ni-only) and Condition $\mathrm{C}$ (Pd-only) over time. 


\subsubsection{Iodine Quenching Experiments for the Reaction of $p$-Anisyl Triflate (1a) with Phenyl Tosylate}

(2a) under Pd-Only Conditions (Condition D)

Table S16. Concentrations of $\mathbf{8}$ and $\mathbf{9}$ and the [aryl iodide]/[Pd] ratios from iodine quenching experiments of Condition $\mathrm{D}^{a, b}$

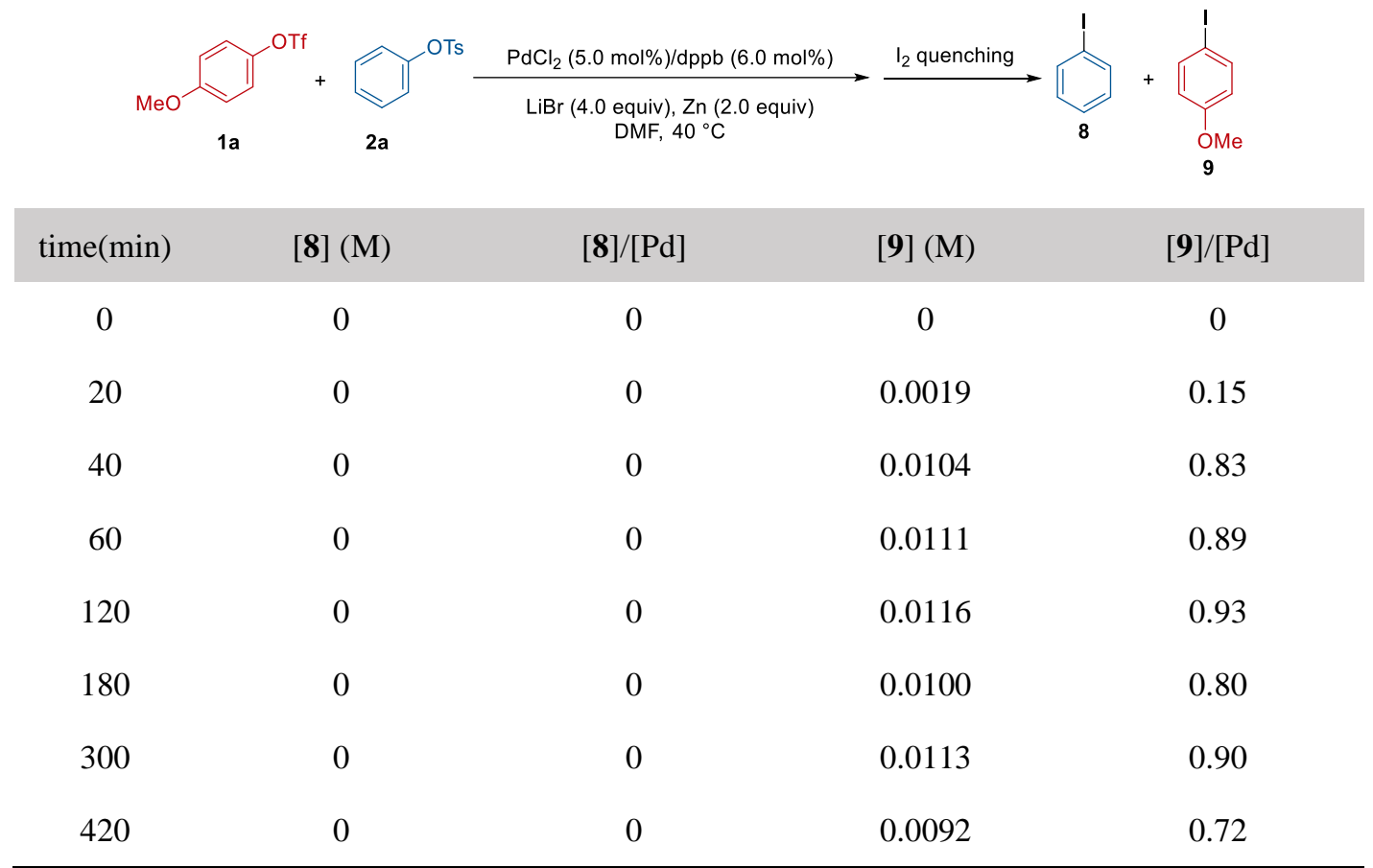

${ }^{a}$ Concentrations determined by GC analysis using 1,3,5-trimethoxybenzene as an internal standard. ${ }^{b}$ Until 420 min, no formation of biaryls or consumption of $\mathbf{2 a}$ was observed. Only trace amount of 1a was consumed.

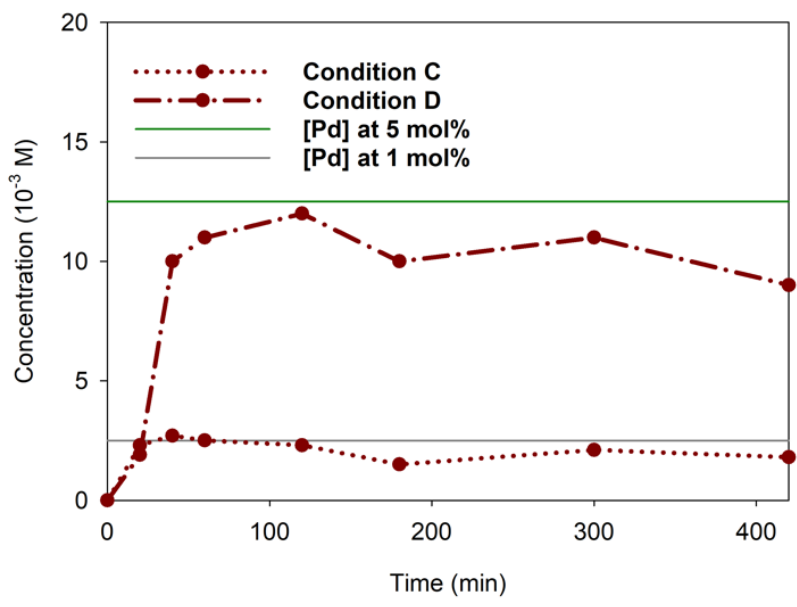

Figure S13. Plot of concentration of $p$-iodoanisole (9) from iodine-quenched aliquots of Condition C and Condition D over time. 


\subsection{Competitive Oxidative Additions of $p$-Anisyl Triflate (1a) and Phenyl Tosylate (2a) to (L1) $\mathrm{Ni}^{0}($ cod $)$}

\section{Procedure}

In a $\mathrm{N}_{2}$ filled glovebox, an oven-dried 1-dram vial equipped with a PTFE-coated stir-bar was charged with $\mathrm{Ni}(\mathrm{COD})_{2}(13.8 \mathrm{mg}, 0.050 \mathrm{mmol})$ and 4,4'-diphenyl-2,2'-bipyridine (15.4 $\left.\mathrm{mg}, 0.050 \mathrm{mmol}\right)$. DMF (1.0 mL) was then added. The vial was capped with a screw cap fitted with a PTFE-faced silicone septum, and the mixture was stirred $(1000 \mathrm{rpm})$ at $\mathrm{rt}$ in the glovebox for $6 \mathrm{~h}$. To the resulting dark blue solution was added 1a (38 mg, $0.15 \mathrm{mmol}), \mathbf{2 a}(37 \mathrm{mg}, 0.15 \mathrm{mmol})$ and 1,3,5-trimethoxybenzene (16.5 $\mathrm{mg})$ as an internal standard. The reaction vial was re-capped with a screw cap fitted with a PTFE-faced silicone septum, and the mixture was heated in a reaction block set to $40{ }^{\circ} \mathrm{C}$ with stirring $(1250 \mathrm{rpm})$. At $5,10,20$ and $30 \mathrm{~min}$, a $50 \mu \mathrm{L}$ aliquot of the reaction mixture was transferred into another 1-dram vial. The vial was capped with a screw cap fitted with a PTFE-faced silicone septum and removed from the glovebox. The aliquot was then diluted with ethyl acetate $(2 \mathrm{~mL})$ and filtered through a $2-\mathrm{cm}$ silica gel plug in a Pasteur pipette. The resulting filtrate was collected in a GC vial. The sample was analyzed by GC and the yields were determined based on the peak area of the analyte compared to 1,3,5-trimethoxybenzene as an internal standard. For the calculations in Table S14, 1a and 2a were used as limiting reagents and the theoretical yield is $0.15 \mathrm{mmol}$.

Table S17. Oxidative additions of $\mathbf{1 a}$ and $\mathbf{2 a}$ to $(\mathbf{L 1}) \mathrm{Ni}^{0}(\operatorname{cod})$ in DMF at $40{ }^{\circ} \mathrm{C}^{a}$

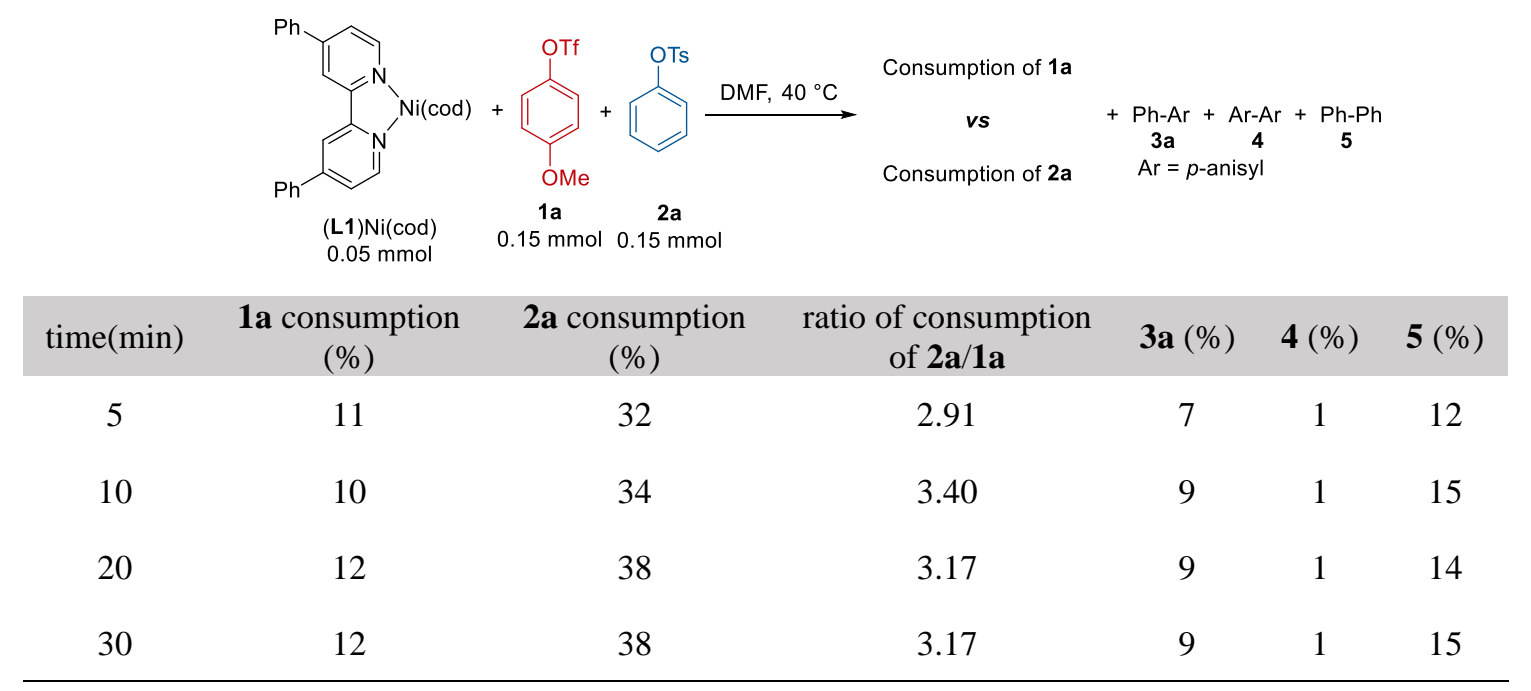

average ratio of consumption of $\mathbf{2 a} / \mathbf{1 a}=3.2 \pm 0.2$

${ }^{a}$ GC yields using 1, 3, 5-trimethoxybenzene as an internal standard. 


\section{References}

1. Yu, P.; Morandi, B. Nickel-Catalyzed Cyanation of Aryl Chlorides and Triflates Using Butyronitrile: Merging Retro-Hydrocyanation with Cross-Coupling. Angew. Chem. Int. Ed. 2017, 56, 15693-15697.

2. Joseph, J. T.; Sajith, A. M.; Ningegowda, R. C.; Shashikanth, S. Room Temperature Carbonylation of (Hetero) Aryl Pentafluoro-Benzenesulfonates and Triflates Using Palladium-Cobalt Bimetallic Catalyst: Dual Role of Cobalt Carbonyl. Adv. Synth. Catal. 2017, 359, 419-425.

3. Komami, N.; Matsuoka, K.; Yoshino, T.; Matsunaga, S., Palladium-Catalyzed Germylation of Aryl Bromides and Aryl Triflates Using Hexamethyldigermane. Synthesis 2018, 50, 2067-2075.

4. Mills, L. R.; Graham, J. M.; Patel, P.; Rousseaux, S. A. L. Ni-Catalyzed Reductive Cyanation of Aryl Halides and Phenol Derivatives Via Transnitrilation. J. Am. Chem. Soc. 2019, 141, 19257-19262.

5. Chang, J. W. W.; Chia, E. Y. R.; Chai, C. L. L.; Seayad, J. Scope of Direct Arylation of Fluorinated Aromatics with Aryl Sulfonates. Org. Biomol. Chem. 2012, 10, 2289-2299.

6. Mori, A.; Mizusaki, T.; Ikawa, T.; Maegawa, T.; Monguchi, Y.; Sajiki, H. Mechanistic Study of a Pd/CCatalyzed Reduction of Aryl Sulfonates Using the Mg-MeOH-NH $4 \mathrm{OAc}$ System. Chem. Eur. J. 2007, 13, 1432-1441.

7. Fawcett, A.; Biberger, T.; Aggarwal, V. K. Carbopalladation of C-C $\sigma$-Bonds Enabled by Strained Boronate Complexes. Nat. Chem. 2019, 11, 117-122.

8. Huang, Z. Y.; Liu, Z.; Zhou, J. R. An Enantioselective, Intermolecular $\alpha$-Arylation of Ester Enolates to Form Tertiary Stereocenters. J. Am. Chem. Soc. 2011, 133, 15882-15885.

9. Alessi, M.; Larkin, A. L.; Ogilvie, K. A.; Green, L. A.; Lai, S.; Lopez, S.; Snieckus, V. Directed ortho Metalation-Boronation and Suzuki-Miyaura Cross Coupling of Pyridine Derivatives: A One-Pot Protocol to Substituted Azabiaryls. J. Org. Chem. 2007, 72, 1588-1594.

10. Wang, S. S.; Chen, X. Z.; Ao, Q. Q.; Wang, H. F.; Zhai, H. B. Decarboxylative C( $\left(\mathrm{sp}^{3}\right)-\mathrm{C}\left(\mathrm{sp}^{3}\right)$ Coupling for Benzylation of Unstable Ketone Enolates: Synthesis of p-(Acylethyl)phenols. Chem. Commun. 2016, $52,9844-9844$.

11. Estrada, L. A.; Neckers, D. C. Synthesis and Photophysics of Dibenz[a,c]Phenazine Derivatives. Org. Lett. 2011, 13, 3304-3307.

12. Wan, W.; Yang, X. Y.; Smith, R. C. Convenient Synthetic Route to Tetraarylphosphonium Polyelectrolytes via Palladium-Catalyzed P-C Coupling of Aryl Triflates and Diphenylphosphine. J. Polym. Sci., Part A: Polym. Chem. 2017, 55, 1984-1990.

13. Ma, X. S.; Dang, H.; Rose, J. A.; Rablen, P.; Herzon, S. B. Hydroheteroarylation of Unactivated Alkenes Using N-Methoxyheteroarenium Salts. J. Am. Chem. Soc. 2017, 139, 5998-6007.

14. O' Neill, P. M.; Stocks, P. A.; Sabbani, S.; Roberts, N. L.; Amewu, R. K.; Shore, E. R.; Aljayyoussi, G.; Angulo-Barturen, I.; Belen, M.; Jimenez-Diaz; Bazaga, S. F.; Martinez, M. S.; Campo, B.; Sharma, R.; Charman, S. A.; Ryan, E.; Chen, G.; Shackleford, D. M.; Davies, J.; Nixon, G. L.; Biagini, G. A.; Ward, S. A. Synthesis and Profiling of Benzylmorpholine 1,2,4,5-Tetraoxane Analogue N205: Towards Tetraoxane Scaffolds with Potential for Single Dose Cure of Malaria. Biorg. Med. Chem. 2018, 26, 2996-3005.

15. Cui, L. C.; Zhang, Z. Q.; Lu, X.; Xiao, B.; Fu, Y. Pd-Catalyzed Cross-Coupling of 1,1-Diborylalkanes with Aryl Triflates. Rsc. Adv. 2016, 6, 51932-51935.

16. Rebih, F.; Andreini, M.; Moncomble, A.; Harrison-Marchand, A.; Maddaluno, J.; Durandetti, M. Direct Carboxylation of Aryl Tosylates by $\mathrm{CO}_{2}$ Catalyzed by in situ-Generated $\mathrm{Ni}^{0}$. Chem. Eur. J. 2016, 22, 3758-3763.

17. Russell, J. E. A.; Entz, E. D.; Joyce, I. M.; Neufeldt, S. R. Nickel-Catalyzed Stille Cross Coupling of C-O Electrophiles. ACS Catal. 2019, 9, 3304-3310.

18. Lei, X. Y.; Jalla, A.; Abou Shama, M. A.; Stafford, J. M.; Cao, B. Chromatography-Free and EcoFriendly Synthesis of Aryl Tosylates and Mesylates. Synthesis 2015, 47, 2578-2585.

19. Ge, Q. Q.; Qian, J. S.; Xuan, J., Electron Donor-Acceptor Complex Enabled Decarboxylative Sulfonylation of Cinnamic Acids under Visible-Light Irradiation. J. Org. Chem. 2019, 84, 8691-8701. 
20. Kuroda, J. I.; Inamoto, K.; Hiroya, K.; Doi, T. N-Heterocyclic Carbene Derived Nickel-Pincer Complexes: Efficient and Applicable Catalysts for Suzuki-Miyaura Coupling Reactions of Aryl/Alkenyl Tosylates and Mesylates. Eur. J. Org. Chem. 2009, 2251-2261.

21. Rostami, A.; Rostami, A.; Ghaderi, A.; Gholinejad, M.; Gheisarzadeh, S. Copper-Catalyzed C-S Bond Formation via the Cleavage of C-O Bonds in the Presence of $\mathrm{S}_{8}$ as the Sulfur Source. Synthesis 2017, 49, 5025-5038.

22. Wang, J. W.; Zhao, J. H.; Gong, H. G. Nickel-Catalyzed Methylation of Aryl Halides/Tosylates with Methyl Tosylate. Chem. Commun. 2017, 53, 10180-10183.

23. Agrawal, T.; Cook, S. P. Iron-Catalyzed Cross-Coupling Reactions of Alkyl Grignards with Aryl Sulfamates and Tosylates. Org. Lett. 2013, 15, 96-99.

24. Nummert, V.; Maemets, V.; Piirsalu, M.; Koppel, I. A. O ${ }^{17}$ NMR Studies of Ortho-Substituent Effects in Substituted Phenyl Tosylates. Magn. Reson. Chem. 2012, 50, 696-704.

25. Lv, L. Y.; Qiu, Z. H.; Li, J. B.; Liu, M. X.; Li, C. J. $\mathrm{N}_{2} \mathrm{H}_{4}$ as Traceless Mediator for Homo- and CrossAryl Coupling. Nat. Commun. 2018, 9, 4739.

26. Yadav, J. S.; Singh, V. K.; Thirupathaiah, B.; Reddy, A. B. First Total Synthesis and Reassignment of Absolute Configuration of Diosniponol A and B. Tetrahedron Lett. 2014, 55, 4427-4429.

27. Aneja, B.; Azam, M.; Alam, S.; Perwez, A.; Maguire, R.; Yadava, U.; Kavanagh, K.; Daniliuc, C. G.; Rizvi, M. M. A.; Haq, Q. M. R.; Abid, M. Natural Product-Based 1,2,3-Triazole/Sulfonate Analogues as Potential Chemotherapeutic Agents for Bacterial Infections. ACS Omega 2018, 3, 6912-6930.

28. Chung, S.; Sach, N.; Choi, C.; Yang, X. J.; Drozda, S. E.; Singer, R. A.; Wright, S. W. Aminocarbonylation of Aryl Tosylates to Carboxamides. Org. Lett. 2015, 17, 2848-2851.

29. Jones, C. S.; Bull, S. D.; Williams, J. M. J. DBN Hexafluorophosphate Salts as Convenient Sulfonylating and Phosphonylating Agents. Org. Biomol. Chem. 2016, 14, 8452-8456.

30. Wilson, D. A.; Wilson, C. J.; Moldoveanu, C.; Resmerita, A. M.; Corcoran, P.; Hoang, L. M.; Rosen, B. M.; Percec, V. Neopentylglycolborylation of Aryl Mesylates and Tosylates Catalyzed by Ni-Based Mixed-Ligand Systems Activated with Zn. J. Am. Chem. Soc. 2010, 132, 1800-1801.

31. Gu, Y. T.; Martin, R. Ni-Catalyzed Stannylation of Aryl Esters via C-O Bond Cleavage. Angew. Chem. Int. Ed. 2017, 56, 3187-3190.

32. Qrareya, H.; Protti, S.; Fagnoni, M. Aryl Imidazylates and Aryl Sulfates as Electrophiles in Metal-Free $\mathrm{ArS}_{\mathrm{N}} 1$ Reactions. J. Org. Chem. 2014, 79, 11527-11533.

33. Durr, A. B.; Yin, G. Y.; Kalvet, I.; Napoly, F.; Schoenebeck, F. Nickel-Catalyzed Trifluoromethylthiolation of Csp²-O Bonds. Chem. Sci. 2016, 7, 1076-1081.

34. Iwai, T.; Harada, T.; Shimada, H.; Asano, K.; Sawamura, M. A Polystyrene-Cross-Linking Bisphosphine: Controlled Metal Monochelation and Ligand-Enabled First-Row Transition Metal Catalysis. ACS Catal. 2017, 7, 1681-1692.

35. Manabe, K.; Kimura, T. Repetitive Synthetic Method for $o, o, p$-Oligophenylenes Using C-H Arylation. Org. Lett. 2013, 15, 374-377.

36. Katritzky, A. R.; Zhang, G. F.; Wu, J. 1-Phenylsulfonylbenzotriazole - a Novel and Convenient Reagent for the Preparation of Benzenesulfonamides and Aryl Benzenesulfonates. Synth. Commun. 1994, 24, 205-216.

37. Alam, M. S.; Koo, S. Deprotection of Durable Benzenesulfonyl Protection for Phenols Efficient Synthesis of Polyphenols. Synth. Commun. 2018, 48, 247-254.

38. Murata, M.; Oda, T.; Sogabe, Y.; Tone, H.; Namikoshi, T.; Watanabe, S. Palladium-Catalyzed Borylation of Aryl Arenesulfonates with Dialkoxyboranes. Chem. Lett. 2011, 40, 962-963.

39. Kageyama, Y.; Ohshima, R.; Sakurama, K.; Fujiwara, Y.; Tanimoto, Y.; Yamada, Y.; Aoki, S. Photochemical Cleavage Reactions of 8-Quinolinyl Sulfonates in Aqueous Solution. Chem. Pharm. Bull. 2009, 57, 1257-1266.

40. Huang, L. B.; Ackerman, L. K. G.; Kang, K.; Parsons, A. M.; Weix, D. J. LiCl-Accelerated Multimetallic Cross-Coupling of Aryl Chlorides with Aryl Triflates. J. Am. Chem. Soc. 2019, 141, 10978-10983. 
41. Liu, W. B.; Li, J. B.; Querard, P.; Li, C. J. Transition-Metal-Free C-C, C-O, and C-N Cross-Couplings Enabled by Light. J. Am. Chem. Soc. 2019, 141, 6755-6764.

42. Liu, X. W.; Zarate, C.; Martin, R. Base-Mediated Defluorosilylation of $\mathrm{C}\left(\mathrm{sp}^{2}\right)-\mathrm{F}$ and $\mathrm{C}\left(\mathrm{sp}^{3}\right)$-F Bonds. Angew. Chem. Int. Ed. 2019, 58, 2064-2068.

43. Ginman, T.; Viklund, J.; Malmstrom, J.; Blid, J.; Emond, R.; Forsblom, R.; Johansson, A.; Kers, A.; Lake, F.; Sehgelmeble, F.; Sterky, K. J.; Bergh, M.; Lindgren, A.; Johansson, P.; Jeppsson, F.; Falting, J.; Gravenfors, Y.; Rahm, F. Core Refinement toward Permeable $\beta$-Secretase (BACE-1) Inhibitors with Low Herg Activity. J. Med. Chem. 2013, 56, 4181-4205.

44. Asghar, S.; Tailor, S. B.; Elorriaga, D.; Bedford, R. B. Cobalt-Catalyzed Suzuki Biaryl Coupling of Aryl Halides. Angew. Chem. Int. Ed. 2017, 56, 16367-16370.

45. Reeves, E. K.; Humke, J. N.; Neufeldt, S. R. N-Heterocyclic Carbene Ligand-Controlled Chemodivergent Suzuki-Miyaura Cross Coupling. J. Org. Chem. 2019, 84, 11799-11812.

46. Wrackmeyer, B. Carbon-13 NMR Spectroscopy of Boron Compounds. Prog. Nucl. Magn. Reson. Spectrosc. 1979, 12, 227-259.

47. Tao, B.; Boykin, D. W. Pd(OAc)2/2-Aryl-2-Oxazolines Catalyzed Suzuki Coupling Reactions of Aryl Bromides and Arylboronic Acids. Tetrahedron Lett. 2002, 43, 4955-4957.

48. Gehrtz, P. H.; Geiger, V.; Schmidt, T.; Srsan, L.; Fleischer, I. Cross-Coupling of Chloro(hetero)arenes with Thiolates Employing a Ni(0)-Precatalyst. Org. Lett. 2019, 21, 50-55.

49. Zhao, Y. G.; Snieckus, V. Beyond Directed Ortho Metalation: Ru-Catalyzed $\mathrm{C}_{\mathrm{Ar}}-\mathrm{O}$ Activation/CrossCoupling Reaction by Amide Chelation. J. Am. Chem. Soc. 2014, 136, 11224-11227.

50. Ando, S.; Matsunaga, H.; Ishizuka, T. An N-Heterocyclic Carbene-Nickel Half-Sandwich Complex as a Precatalyst for Suzuki Miyaura Coupling of Aryl/Heteroaryl Halides with Aryl/Heteroarylboronic Acids. J. Org. Chem. 2017, 82, 1266-1272.

51. Karmakar, S.; Mandal, T.; Dash, J. Ring Closing Metathesis Approach for the Synthesis of $o$-Terphenyl Derivatives. Eur. J. Org. Chem. 2019, 5916-5924.

52. Shieh, W. C.; Carlson, J. A. A Simple Asymmetric-Synthesis of 4-Arylphenylalanines via PalladiumCatalyzed Cross-Coupling Reaction of Arylboronic Acids with Tyrosine Triflate. J. Org. Chem. 1992, 57, 379-381.

53. Drinkwater, N.; Vinh, N. B.; Mistry, S. N.; Bamert, R. S.; Ruggeri, C.; Holleran, J. P.; Loganathan, S.; Paiardini, A.; Charman, S. A.; Powell, A. K.; Avery, V. M.; McGowan, S.; Scammells, P. J. Potent Dual Inhibitors of Plasmodium Falciparum M1 and M17 Aminopeptidases through Optimization of S1 Pocket Interactions. Eur. J. Med. Chem. 2016, 110, 43-64.

54. Ohgi, A.; Semba, K.; Hiyama, T.; Nakao, Y. Silicon-Based Cross-Coupling of Aryl Tosylates by Cooperative Palladium/Copper Catalysis. Chem. Lett. 2016, 45, 973-975.

55. Krasovskiy, A.; Knochel, P. Convenient Titration Method for Organometallic Zinc, Magnesium, and Lanthanide Reagents. Synthesis 2006, 890-891. 
Copies of NMR Spectra 
${ }^{1} \mathrm{H}$ NMR spectrum for compound 3a following general procedure $\mathrm{A}\left(400 \mathrm{MHz}, \mathrm{CDCl}_{3}\right)$

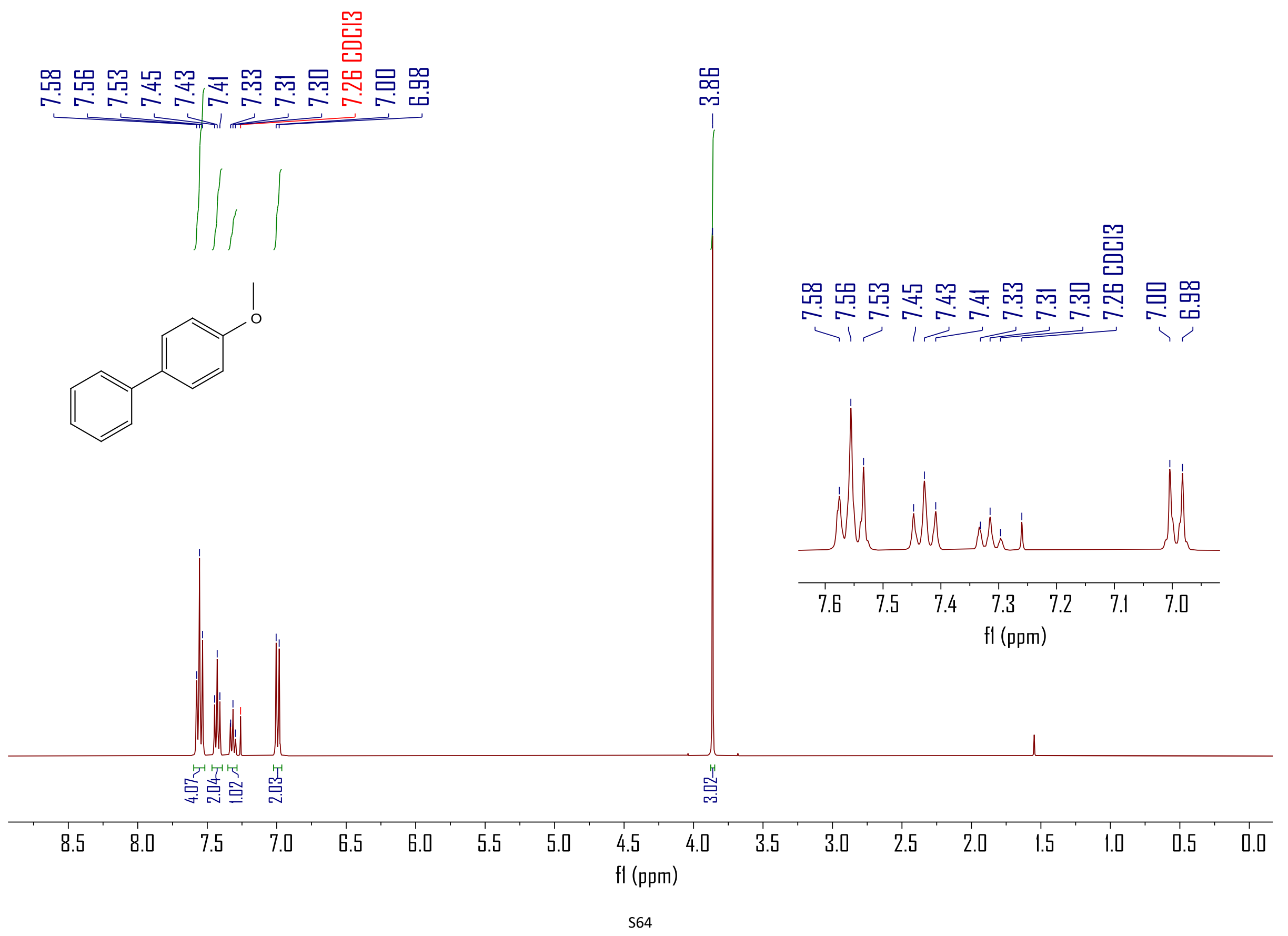


${ }^{13} \mathrm{C}\left\{{ }^{1} \mathrm{H}\right\}$ NMR spectrum for compound 3a following general procedure $\mathrm{A}\left(100 \mathrm{MHz}, \mathrm{CDCl}_{3}\right)$
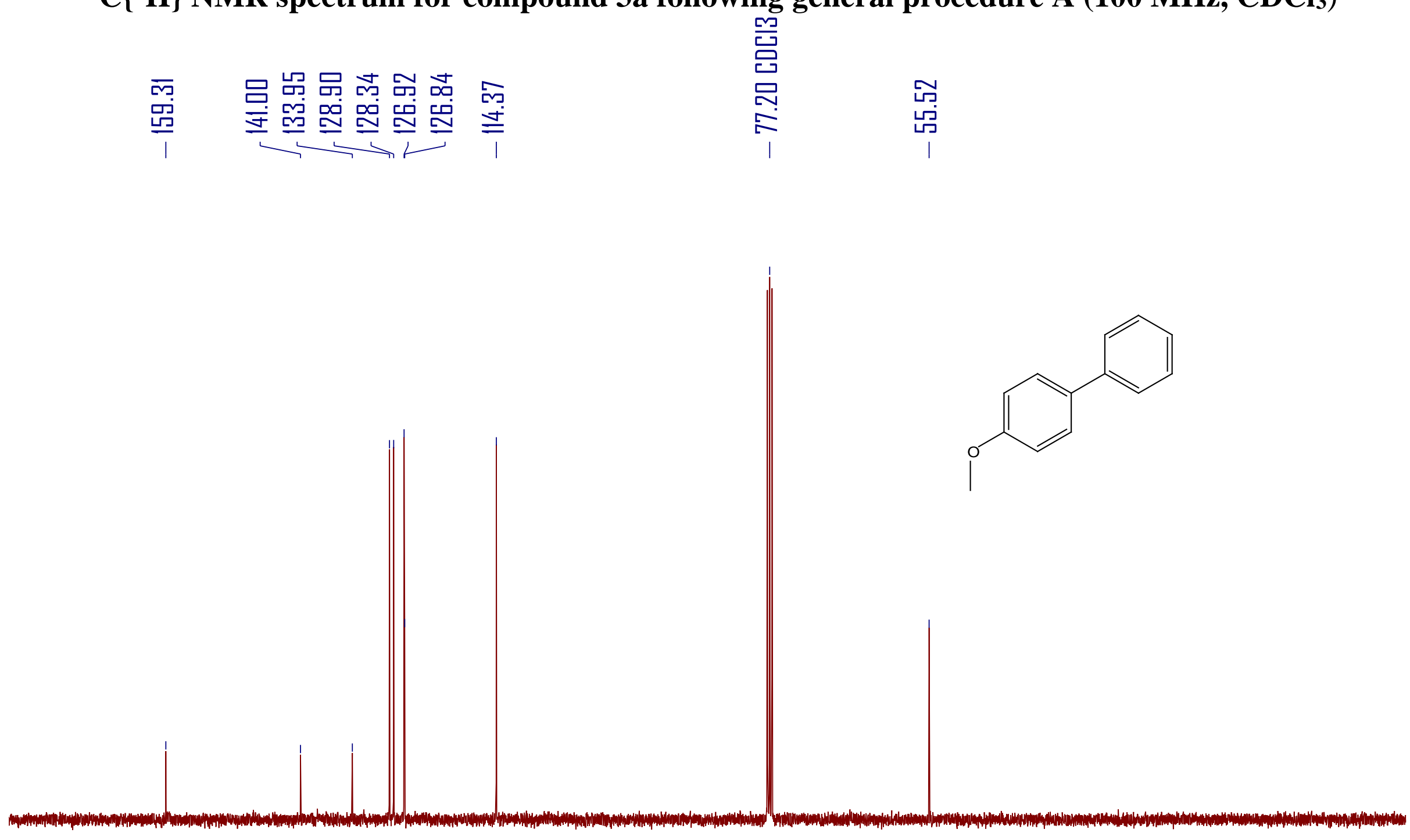

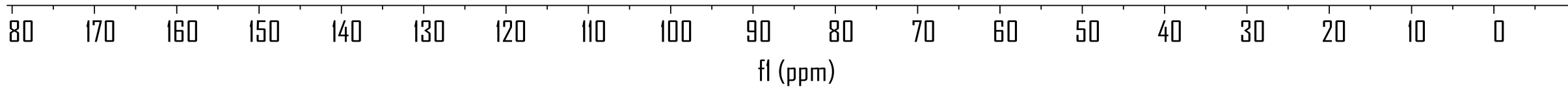


${ }^{1} \mathrm{H}$ NMR spectrum for compound 3a following general procedure $\mathrm{B}\left(400 \mathrm{MHz}, \mathrm{CDCl}_{3}\right)$

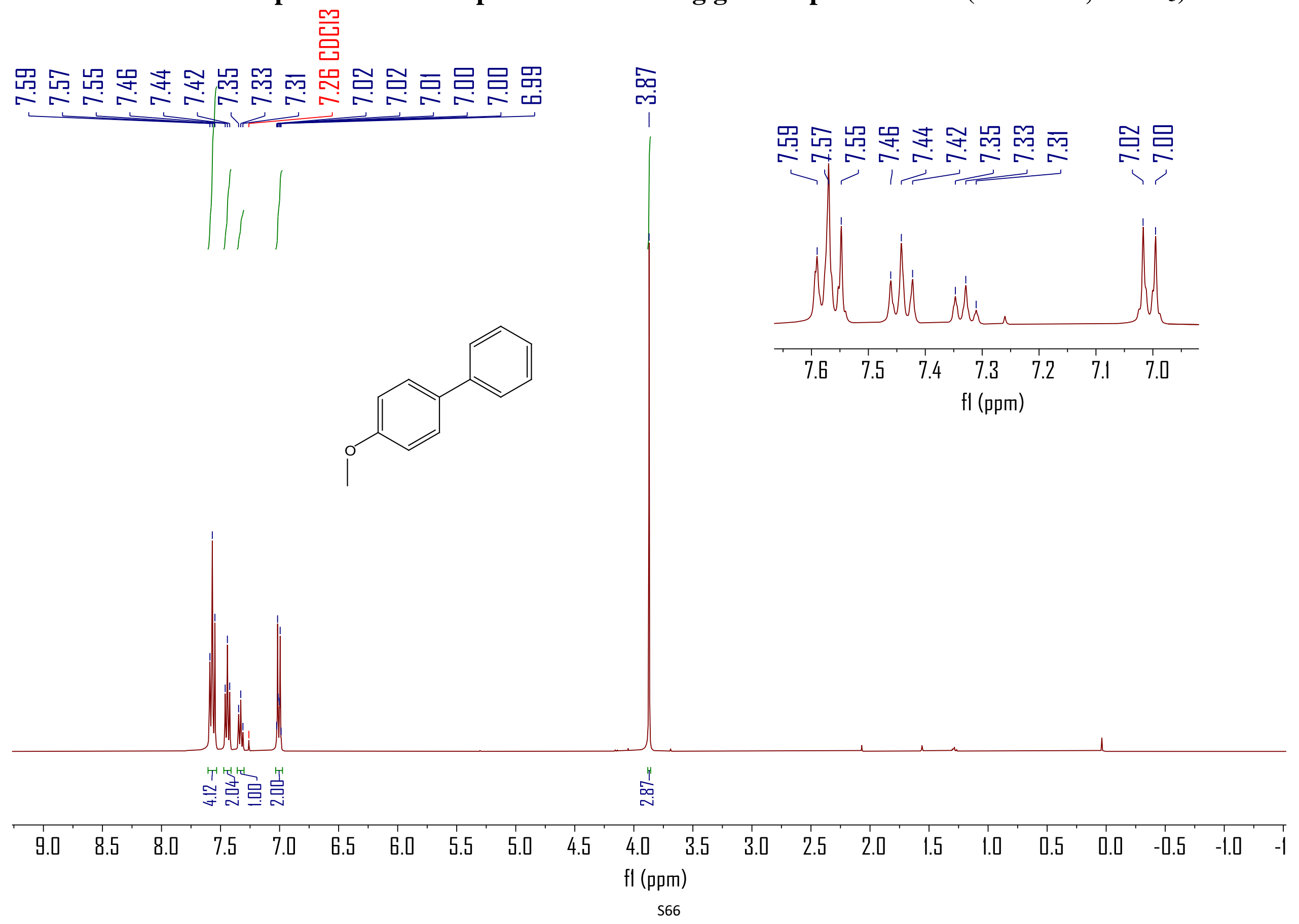


${ }^{13} \mathrm{C}\left\{{ }^{1} \mathrm{H}\right\}$ NMR spectrum for compound 3a following general procedure $\mathrm{B}\left(100 \mathrm{MHz}, \mathrm{CDCl}_{3}\right)$

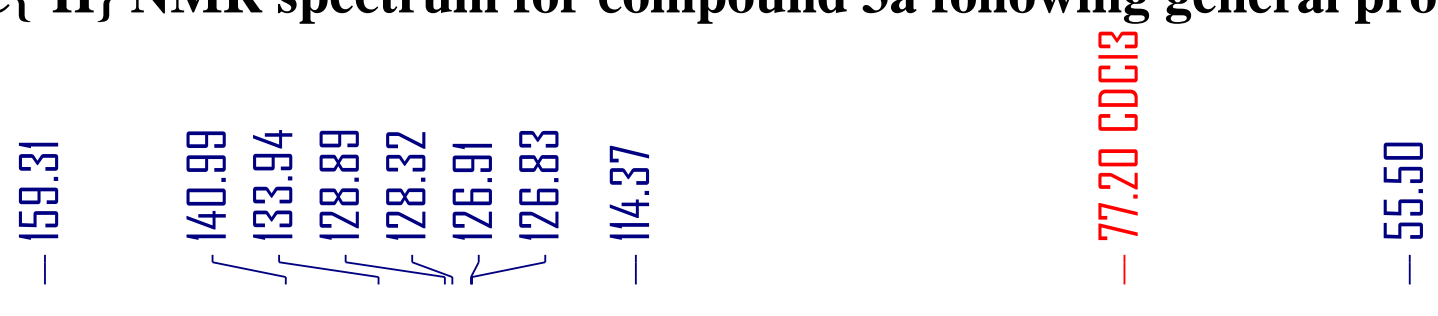

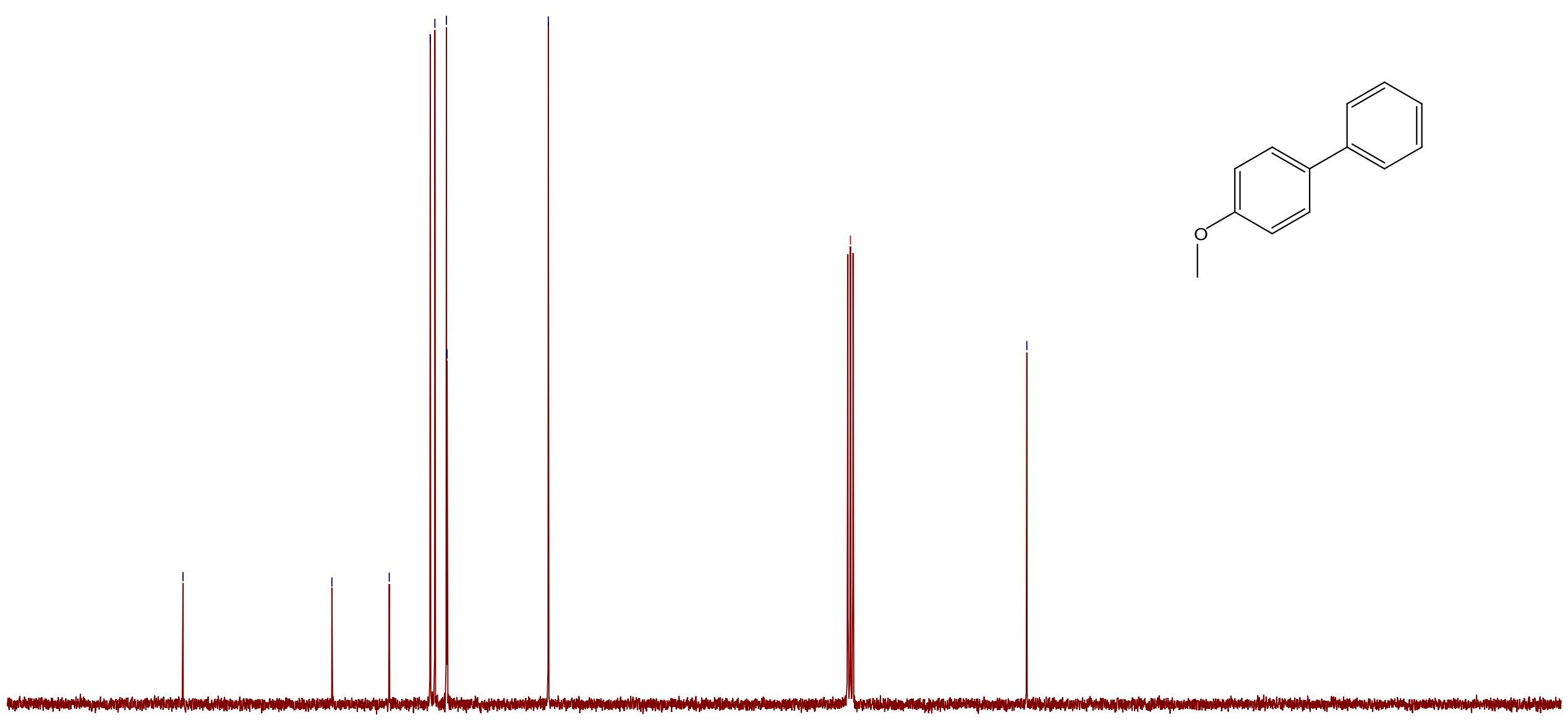

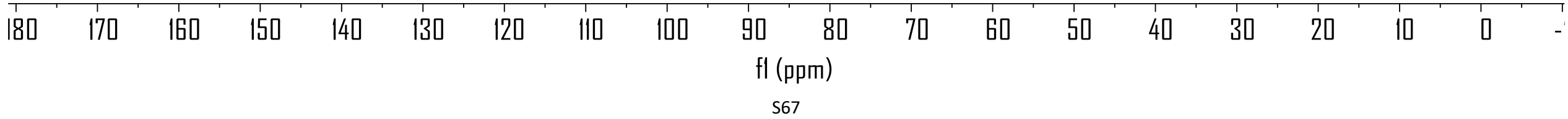




\section{${ }^{1} \mathrm{H}$ NMR spectrum for compound $3 \mathrm{~b}\left(400 \mathrm{MHz}, \mathrm{CDCl}_{3}\right)$}

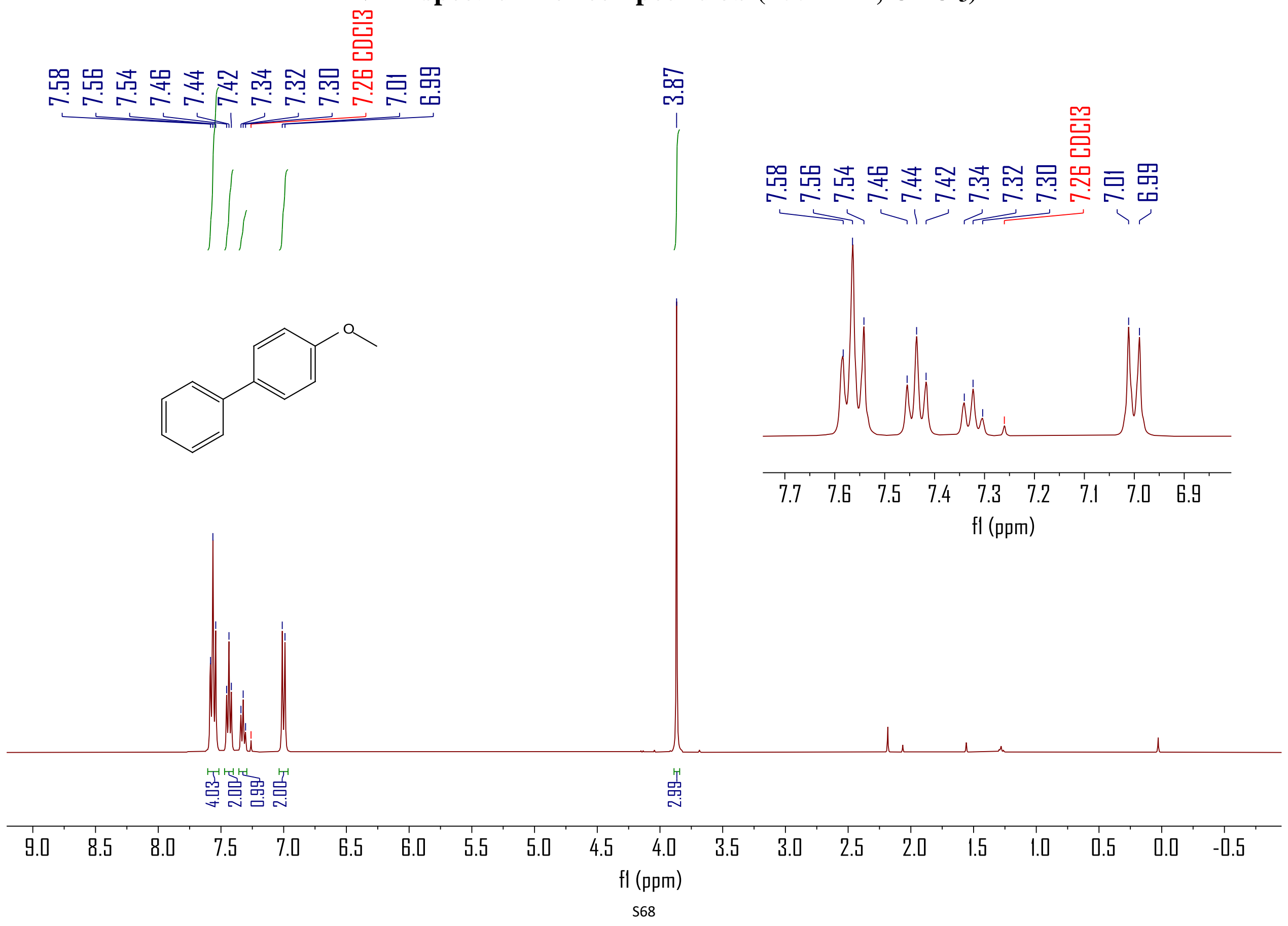




\section{${ }^{13} \mathrm{C}\left\{{ }^{1} \mathrm{H}\right\} \mathrm{NMR}$ spectrum for compound $3 \mathrm{~b}\left(100 \mathrm{MHz}, \mathrm{CDCl}_{3}\right)$}
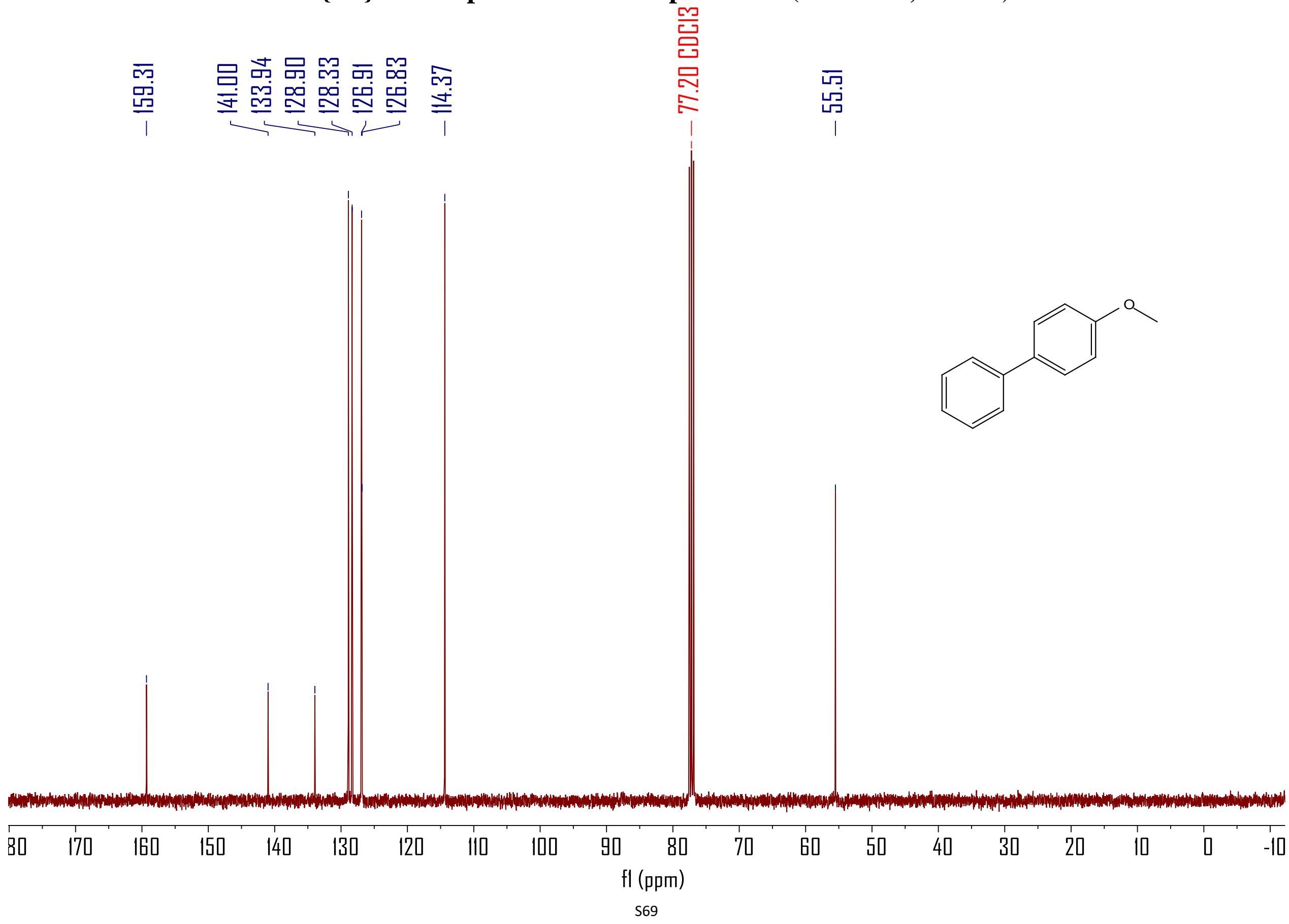


\section{${ }^{1} \mathrm{H}$ NMR spectrum for compound $3 \mathrm{c}\left(500 \mathrm{MHz} \mathrm{CDCl}_{3}\right)$}

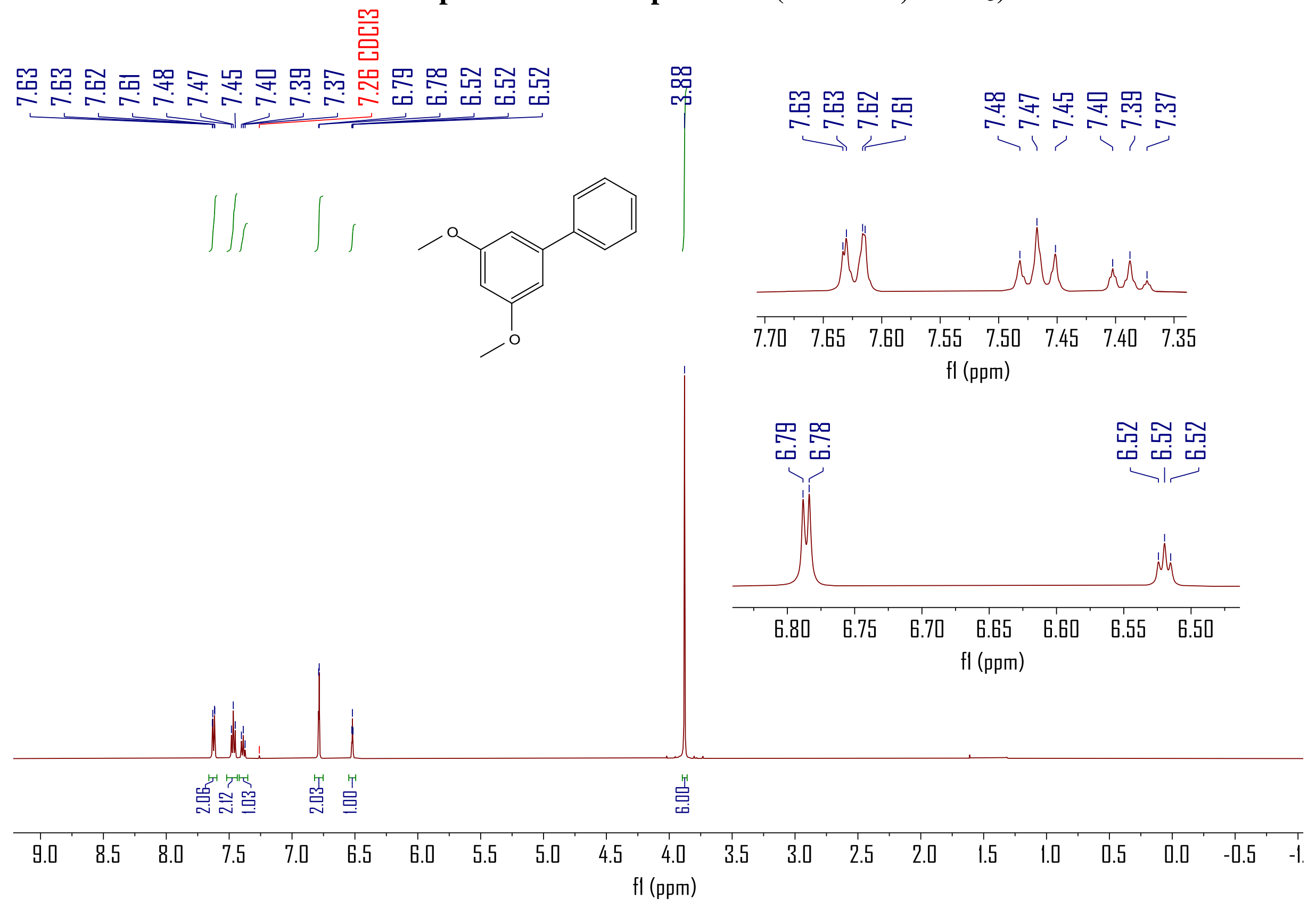




\section{${ }^{13} \mathrm{C}\left\{{ }^{1} \mathrm{H}\right\}$ NMR spectrum for compound $3 \mathrm{c}\left(125 \mathrm{MHz}, \mathrm{CDCl}_{3}\right)$}

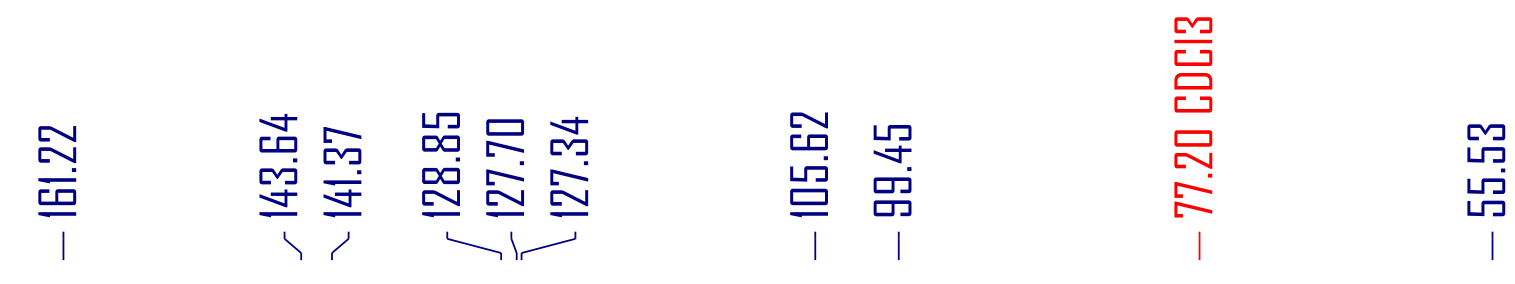

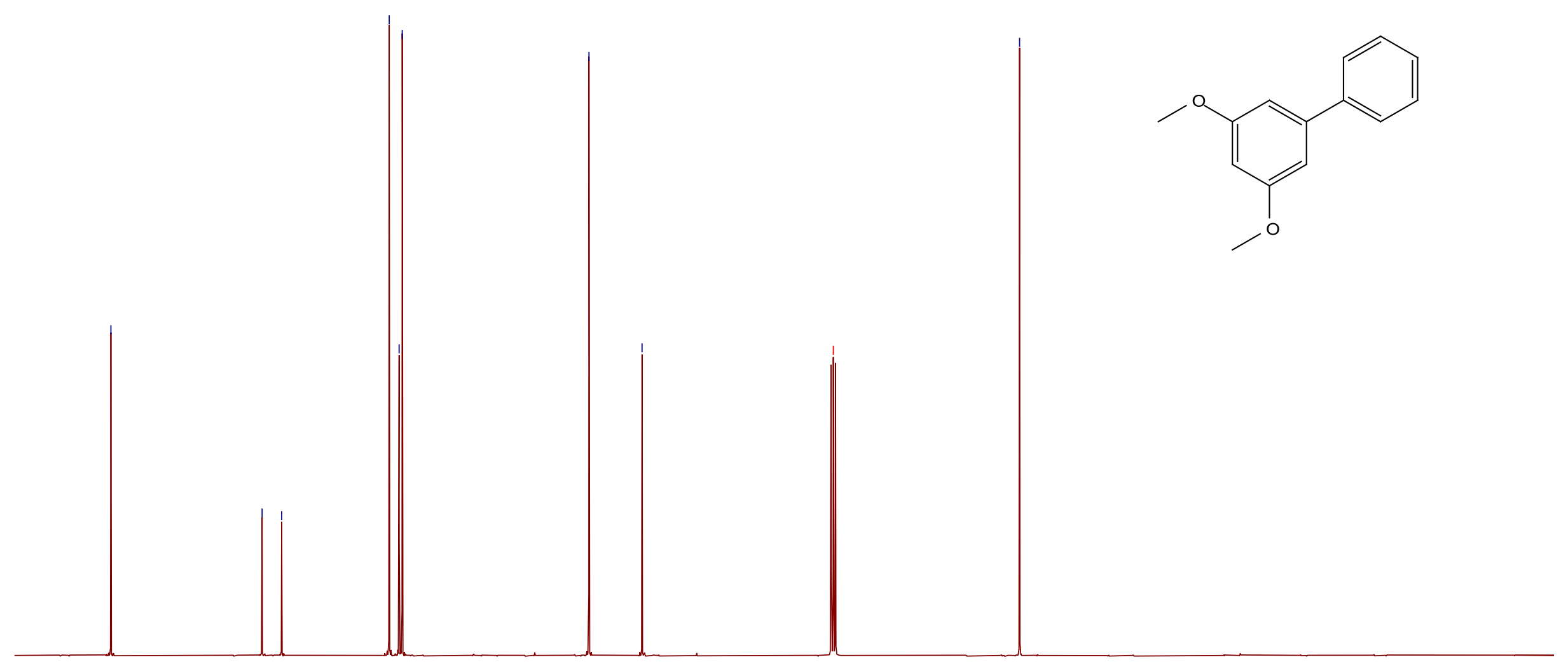

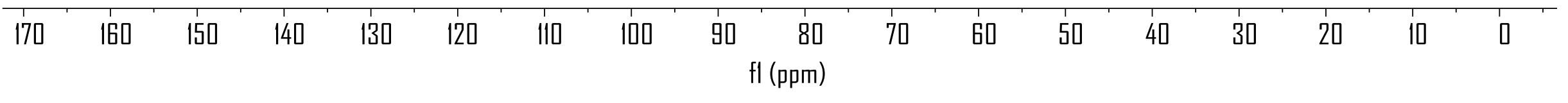




\section{${ }^{1} \mathrm{H}$ NMR spectrum for compound 3d following general procedure $\mathrm{A}\left(400 \mathrm{MHz}, \mathrm{CDCl}_{3}\right)$}

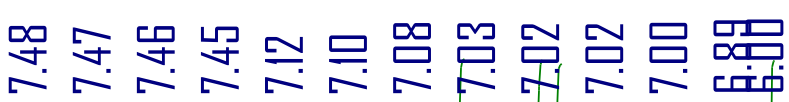
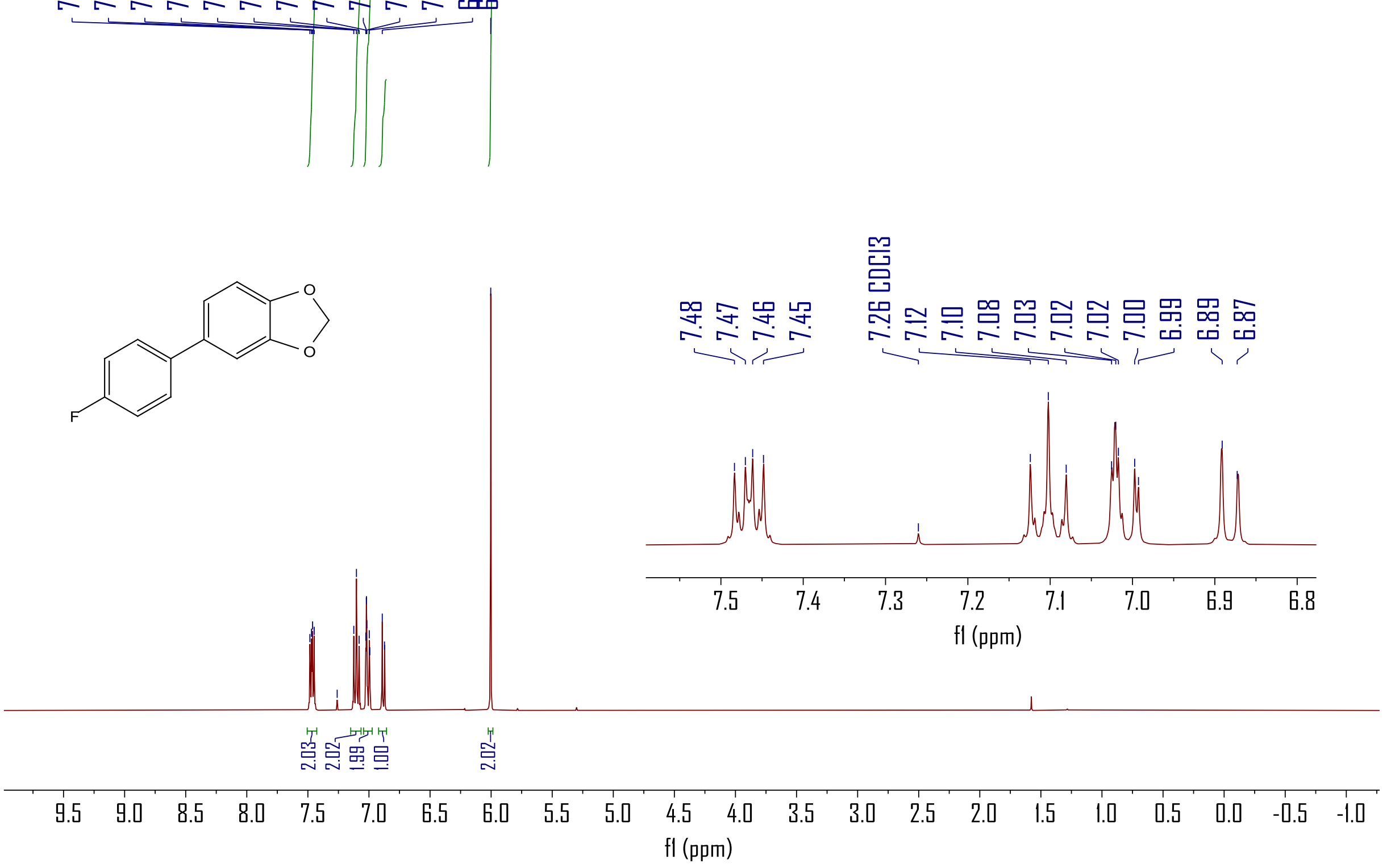


\section{${ }^{13} \mathrm{C}\left\{{ }^{1} \mathrm{H}\right\}$ NMR spectrum for compound 3d following general procedure $\mathrm{A}\left(100 \mathrm{MHz}, \mathrm{CDCl}_{3}\right)$}

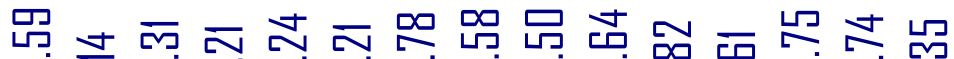

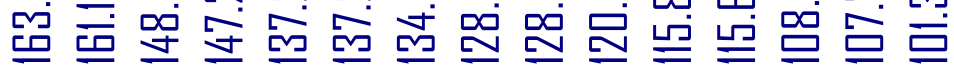

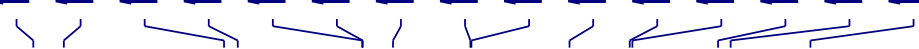
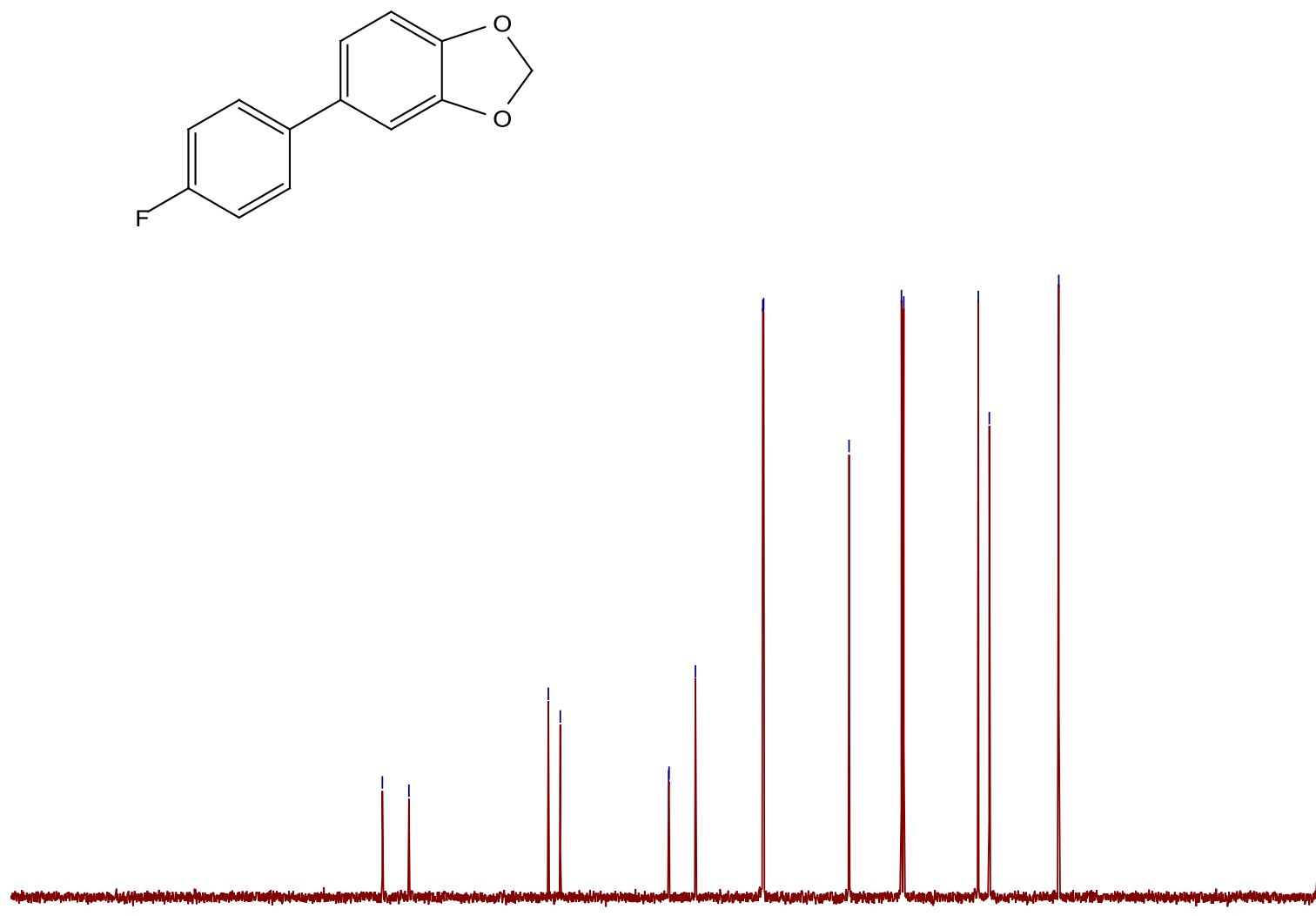

몸

品 号

只 哭

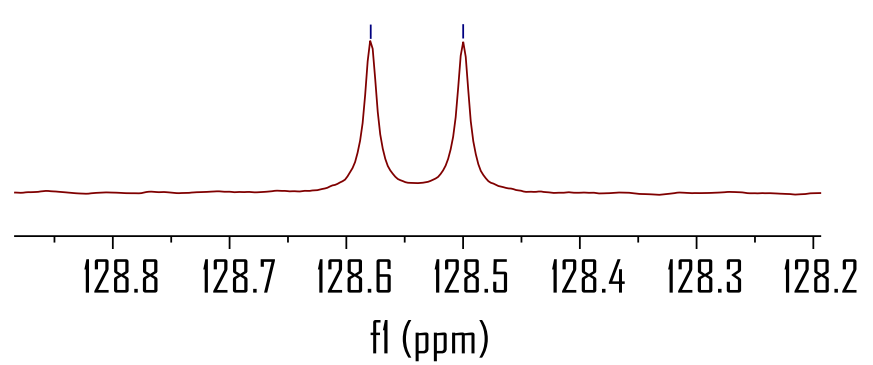

压

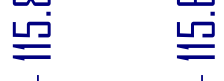

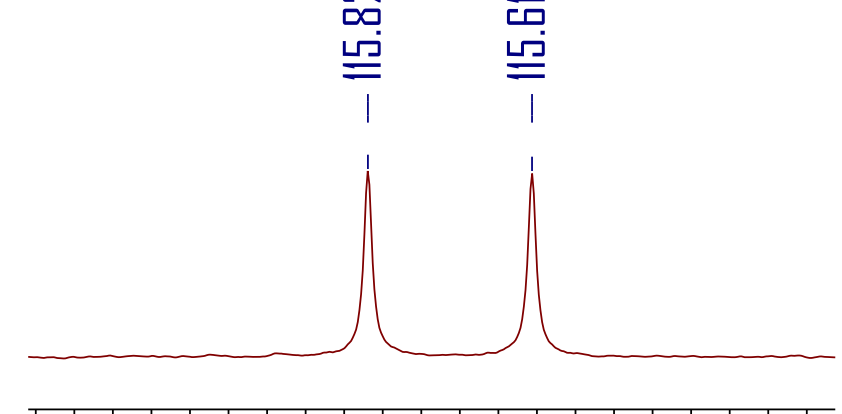

\|6.2 \|6. I I6.

$\mathrm{fl}$ (ppm)

$190 \quad 180 \quad 170 \quad 160$

$150 \quad 140 \quad 130 \quad 120$

$110 \quad 100$

90

80

70

$60 \quad 50$

40

30

$20 \quad 10$ 
${ }^{19} \mathrm{~F}\left\{{ }^{1} \mathrm{H}\right\}$ NMR spectrum for compound 3d following general procedure $\mathrm{A}\left(376 \mathrm{MHz}, \mathrm{CDCl}_{3}\right)$

号

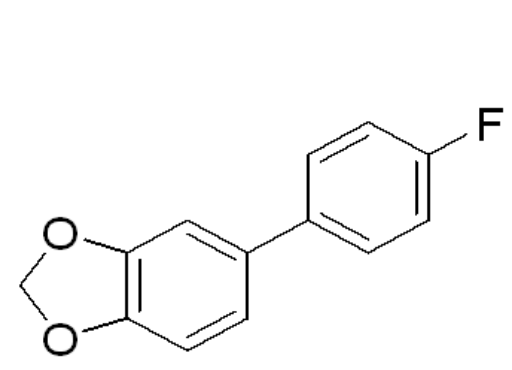
fl (ppm)

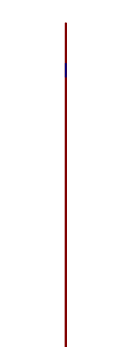


${ }^{1} \mathrm{H}$ NMR spectrum for compound $3 \mathrm{~d}$ following general procedure $\mathrm{B}\left(400 \mathrm{MHz}, \mathrm{CDCl}_{3}\right)$

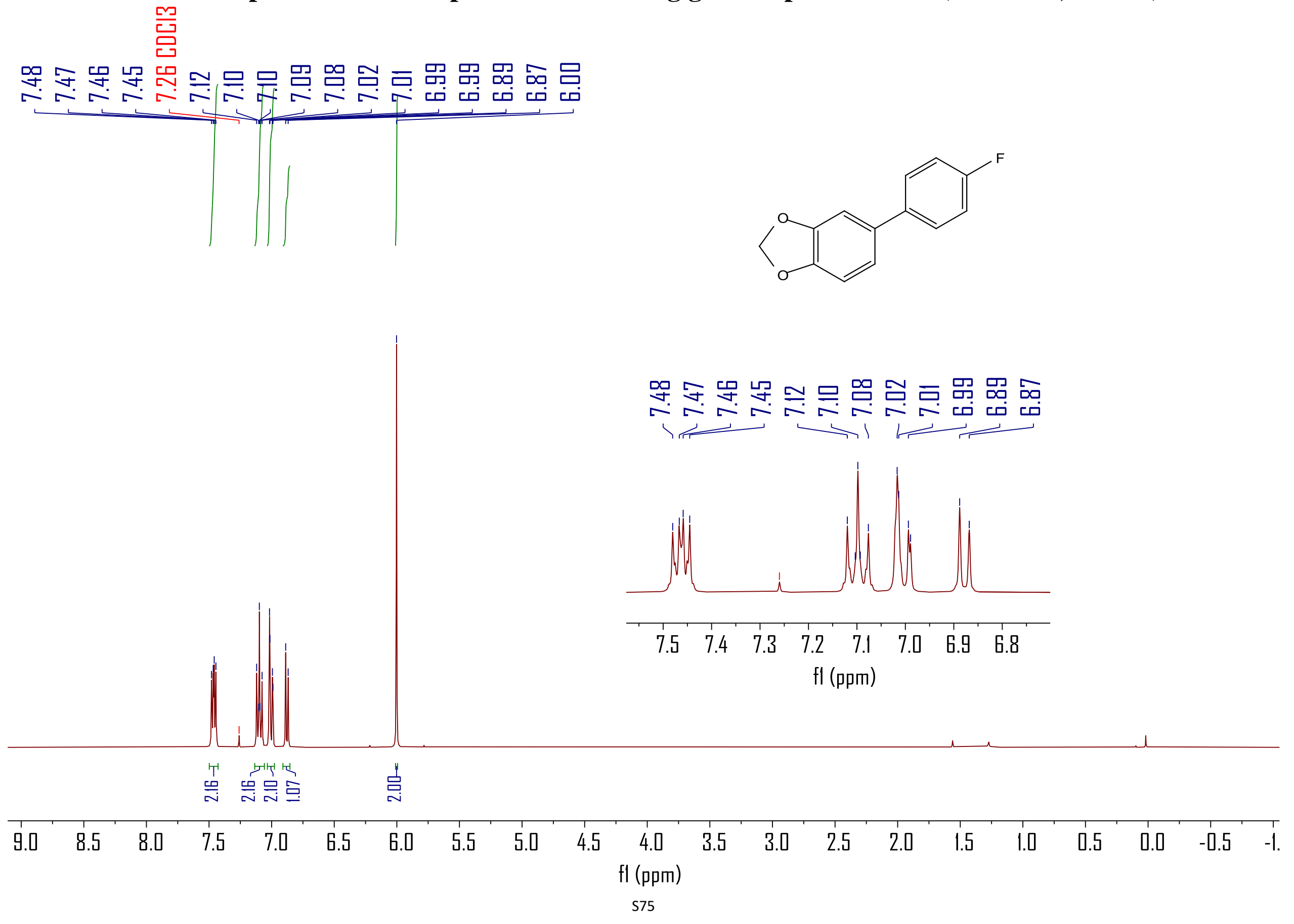




\section{${ }^{13} \mathrm{C}\left\{{ }^{1} \mathrm{H}\right\}$ NMR spectrum for compound 3d following general procedure $\mathrm{B}\left(100 \mathrm{MHz}, \mathrm{CDCl}_{3}\right)$}

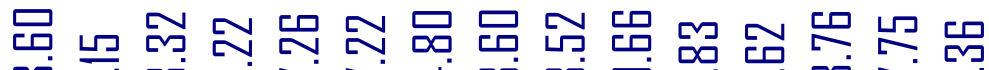

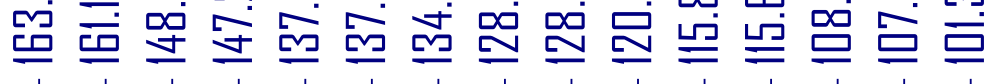

$$
\text { 몸 }
$$
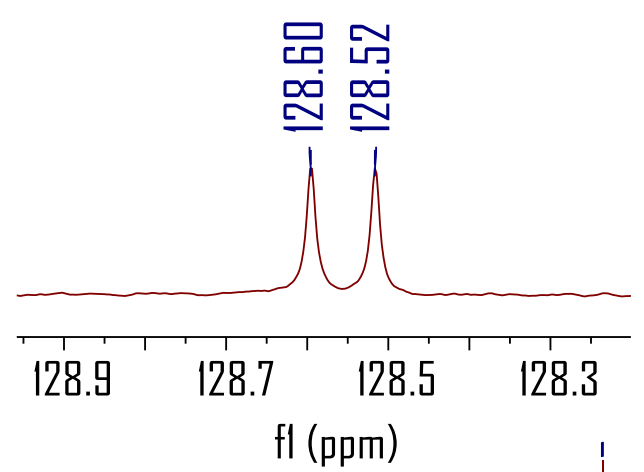

$$
\mathrm{fl}(\mathrm{ppm})
$$

170

$150 \quad 140 \quad 130$

120
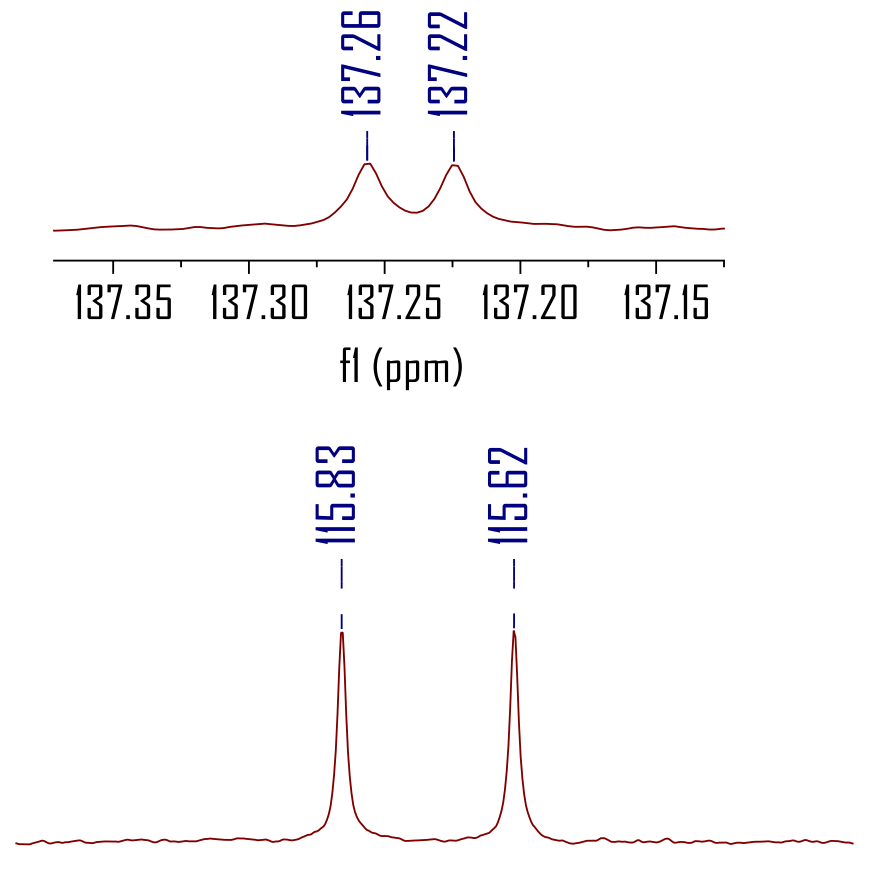

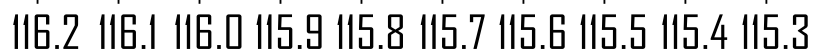
$\mathrm{fl}(\mathrm{ppm})$

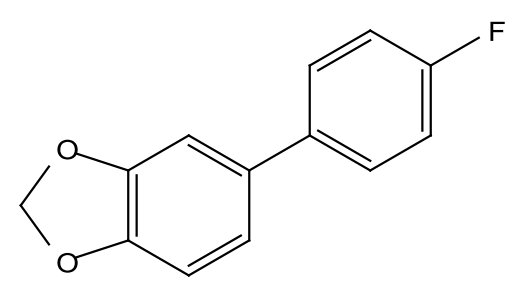


${ }^{19} \mathrm{~F}\left\{{ }^{1} \mathrm{H}\right\}$ NMR spectrum for compound 3d following general procedure $\mathrm{B}\left(376 \mathrm{MHz}, \mathrm{CDCl}_{3}\right)$

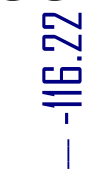

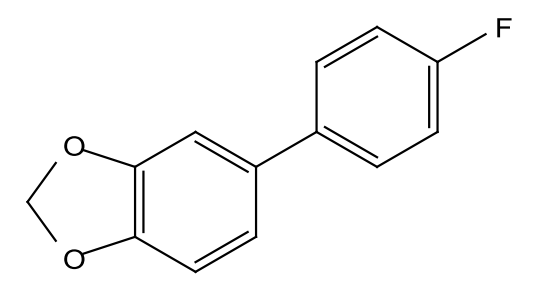

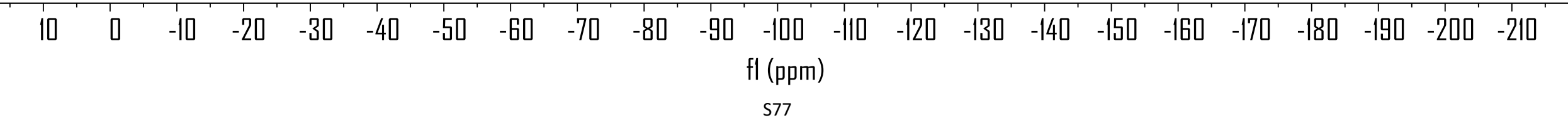




\section{${ }^{1} \mathrm{H}$ NMR spectrum for compound $3 \mathrm{e}\left(400 \mathrm{MHz}, \mathrm{CDCl}_{3}\right)$}

믐

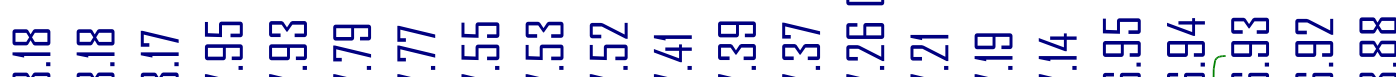

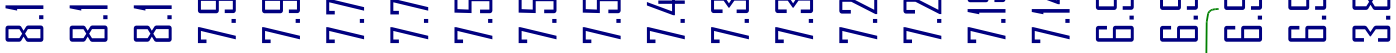
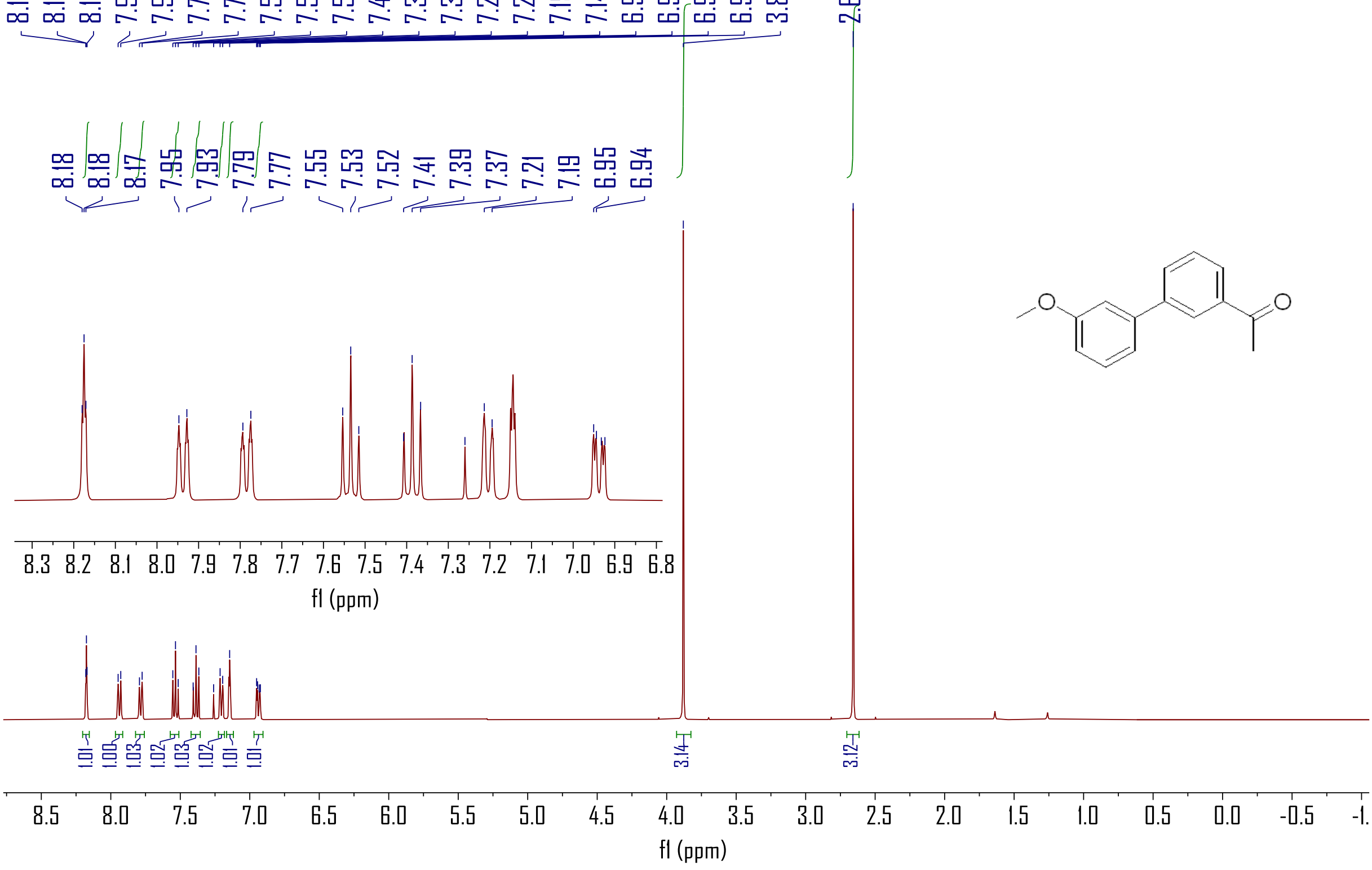


\section{${ }^{13} \mathrm{C}\left\{{ }^{1} \mathrm{H}\right\}$ NMR spectrum for compound $3 \mathrm{e}\left(125 \mathrm{MHz}, \mathrm{CDCl}_{3}\right)$}

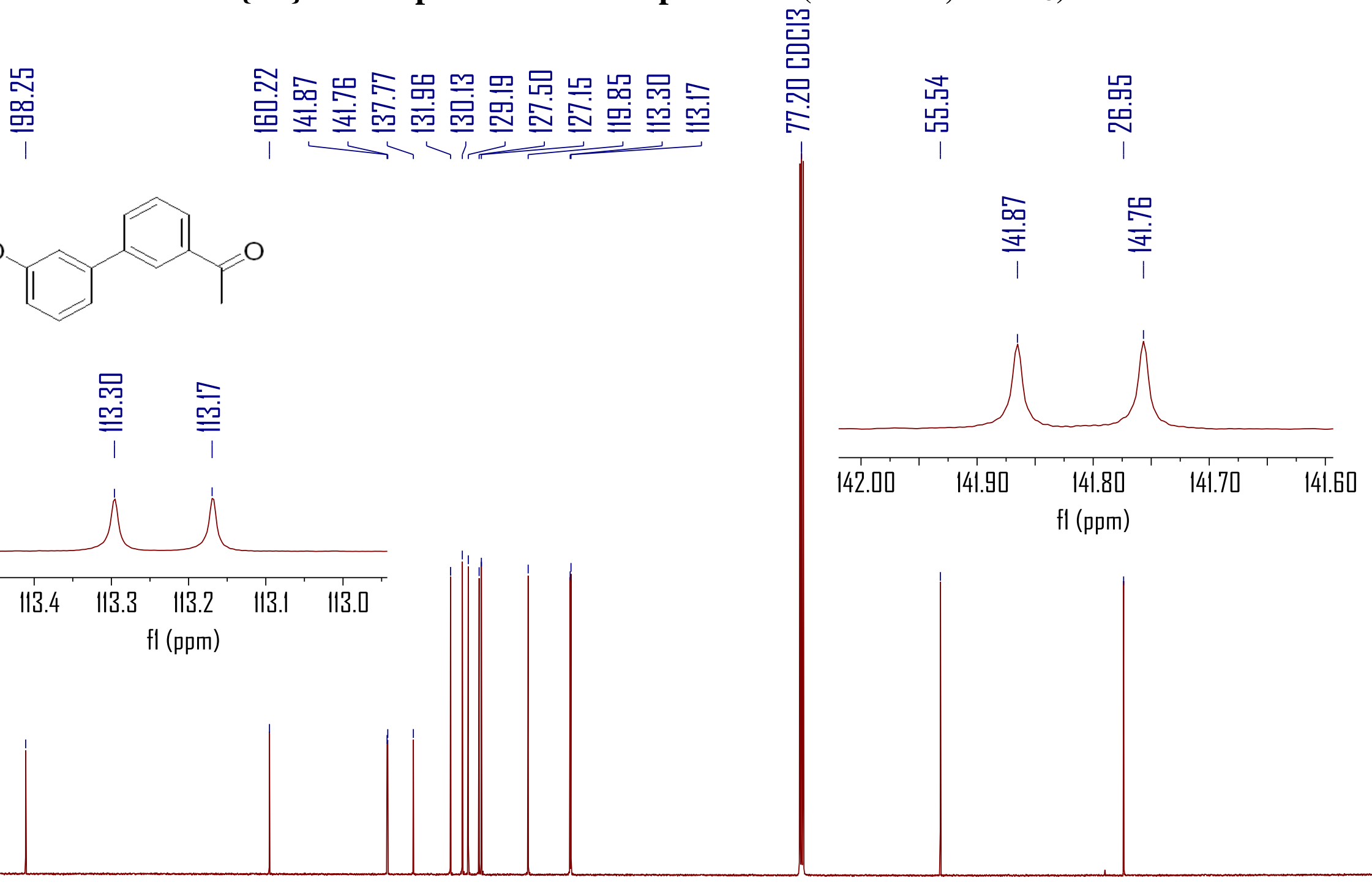

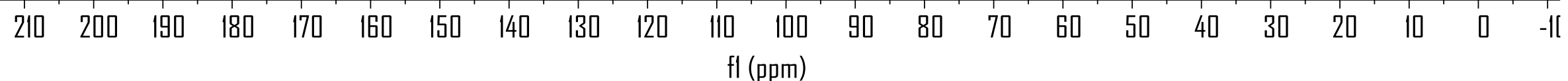




\section{${ }^{1} \mathrm{H}$ NMR spectrum for compound $3 \mathrm{f}\left(500 \mathrm{MHz}, \mathrm{CDCl}_{3}\right)$}

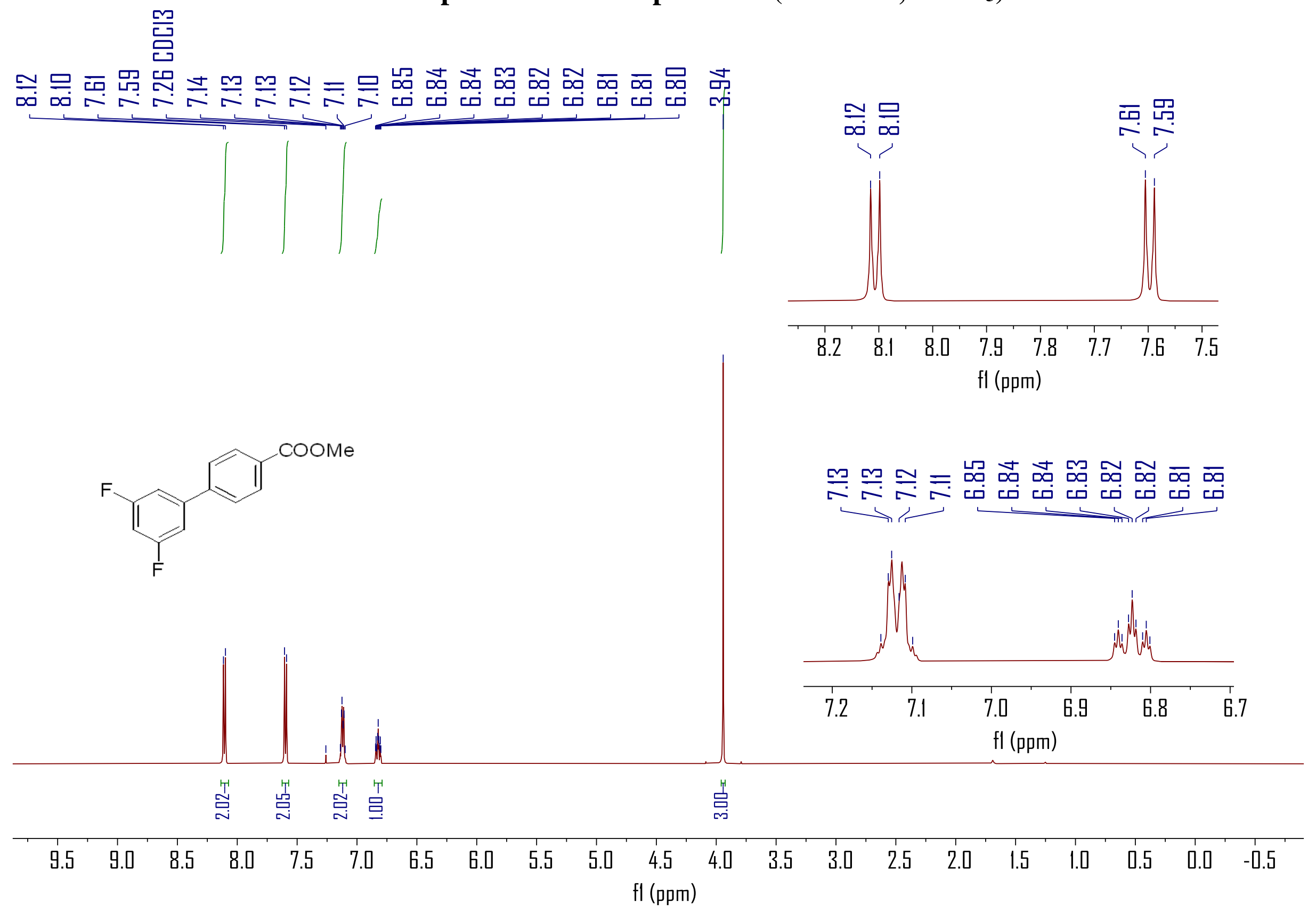




\section{${ }^{13} \mathrm{C}\left\{{ }^{1} \mathrm{H}\right\}$ NMR spectrum for compound $3 f\left(125 \mathrm{MHz}, \mathrm{CDCl}_{3}\right)$}

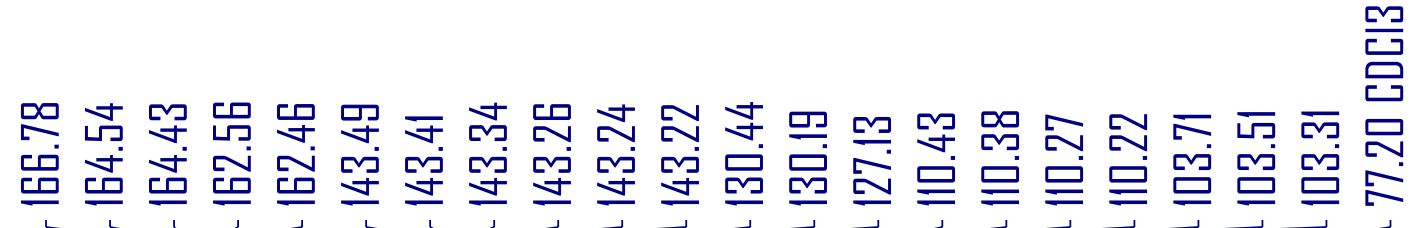

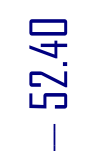

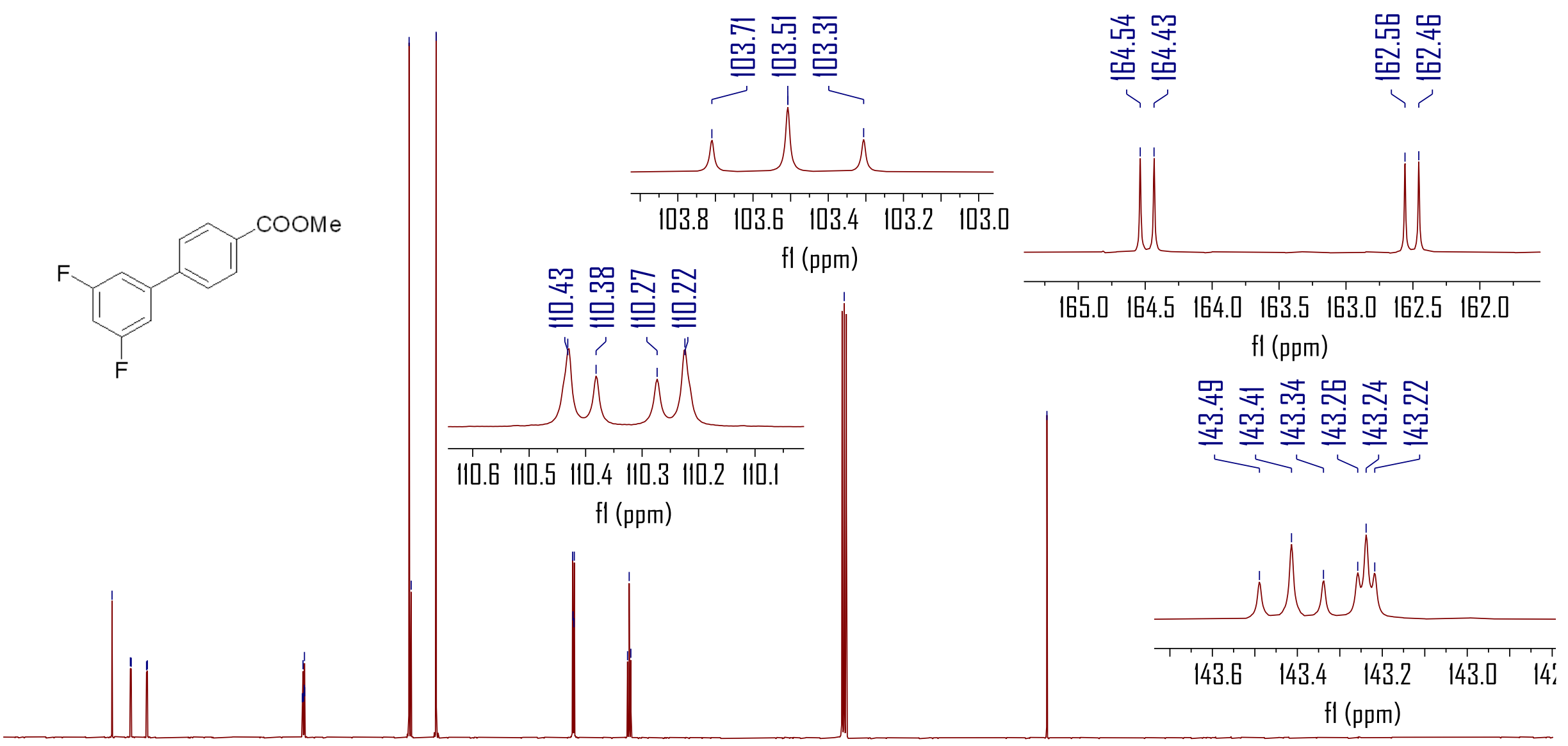

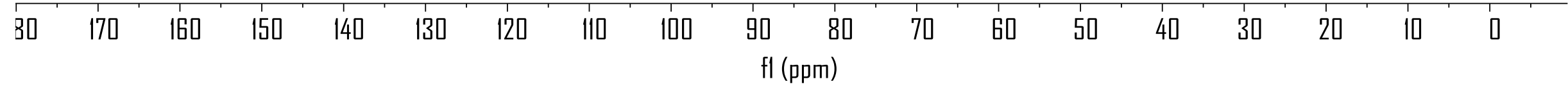




\section{${ }^{19} \mathrm{~F}\left\{{ }^{1} \mathrm{H}\right\}$ NMR spectrum for compound $3 f\left(376 \mathrm{MHz}, \mathrm{CDCl}_{3}\right)$}

$$
\text { 号 }
$$

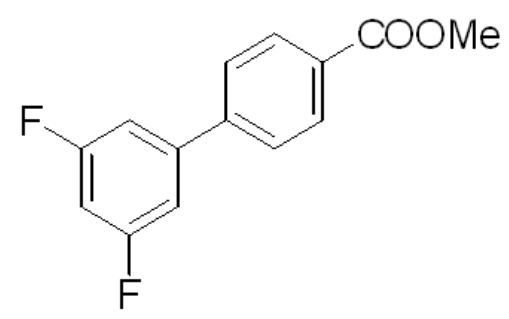

总

$\begin{array}{llllllllllllllllllllllll}10 & 0 & -10 & -20 & -30 & -40 & -50 & -60 & -70 & -80 & -90 & -100 & -110 & -120 & -130 & -140 & -150 & -160 & -170 & -180 & -190 & -200 & -210\end{array}$




\section{${ }^{1} \mathrm{H}$ NMR spectrum for compound $3 \mathrm{~g}\left(400 \mathrm{MHz}, \mathrm{CDCl}_{3}\right)$}
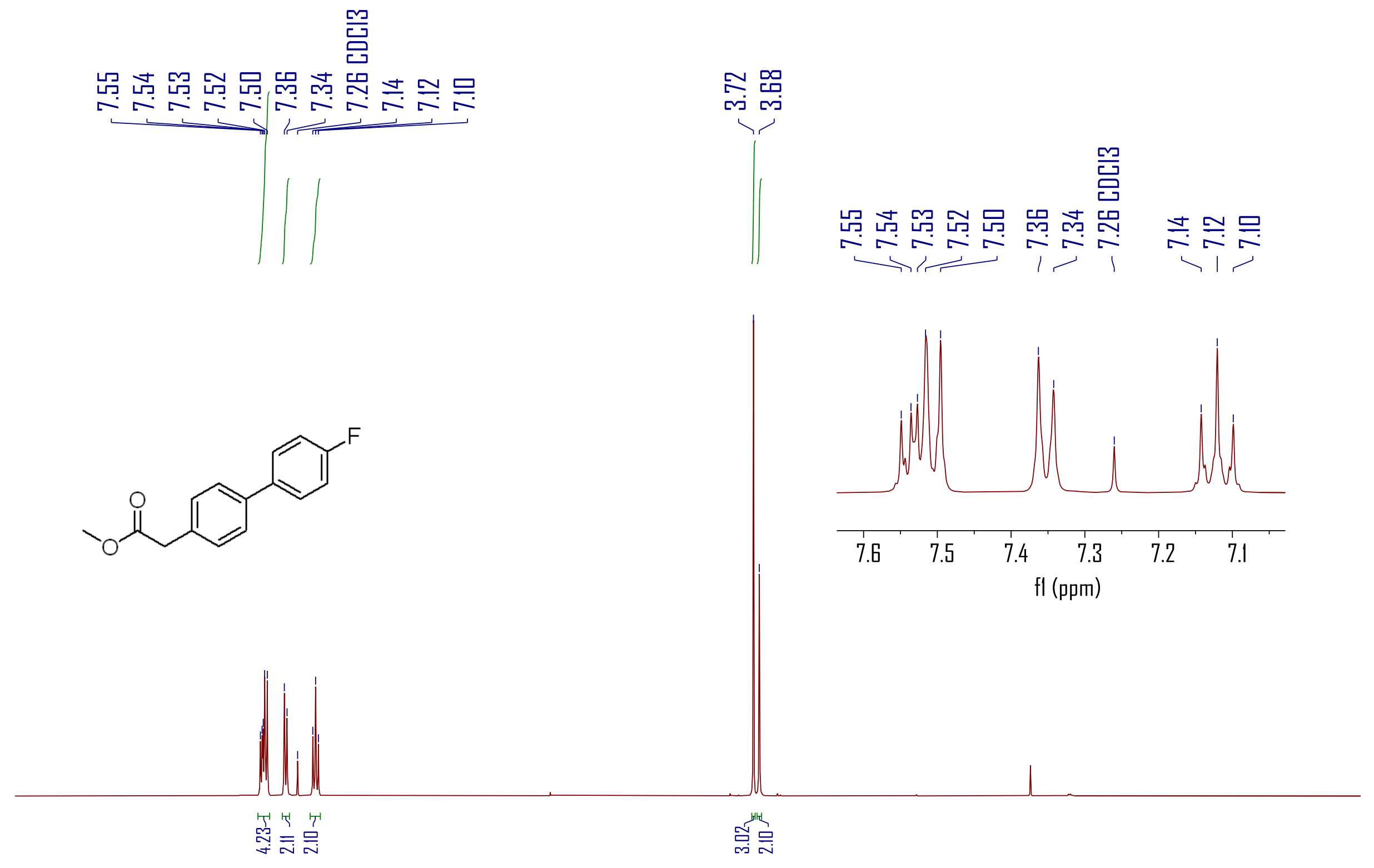

$\begin{array}{llllllll}9.0 & 8.5 & 8.0 & 7.5 & 7.0 & 6.5 & 6.0 & 5.5\end{array}$

$5.5 \quad 5.0 \quad 4.5$




\section{${ }^{13} \mathrm{C}\left\{{ }^{1} \mathrm{H}\right\}$ NMR spectrum for compound $3 \mathrm{~g}\left(125 \mathrm{MHz}, \mathrm{CDCl}_{3}\right)$}

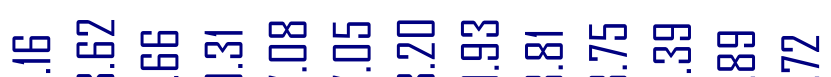

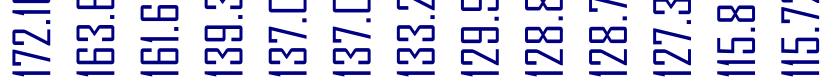
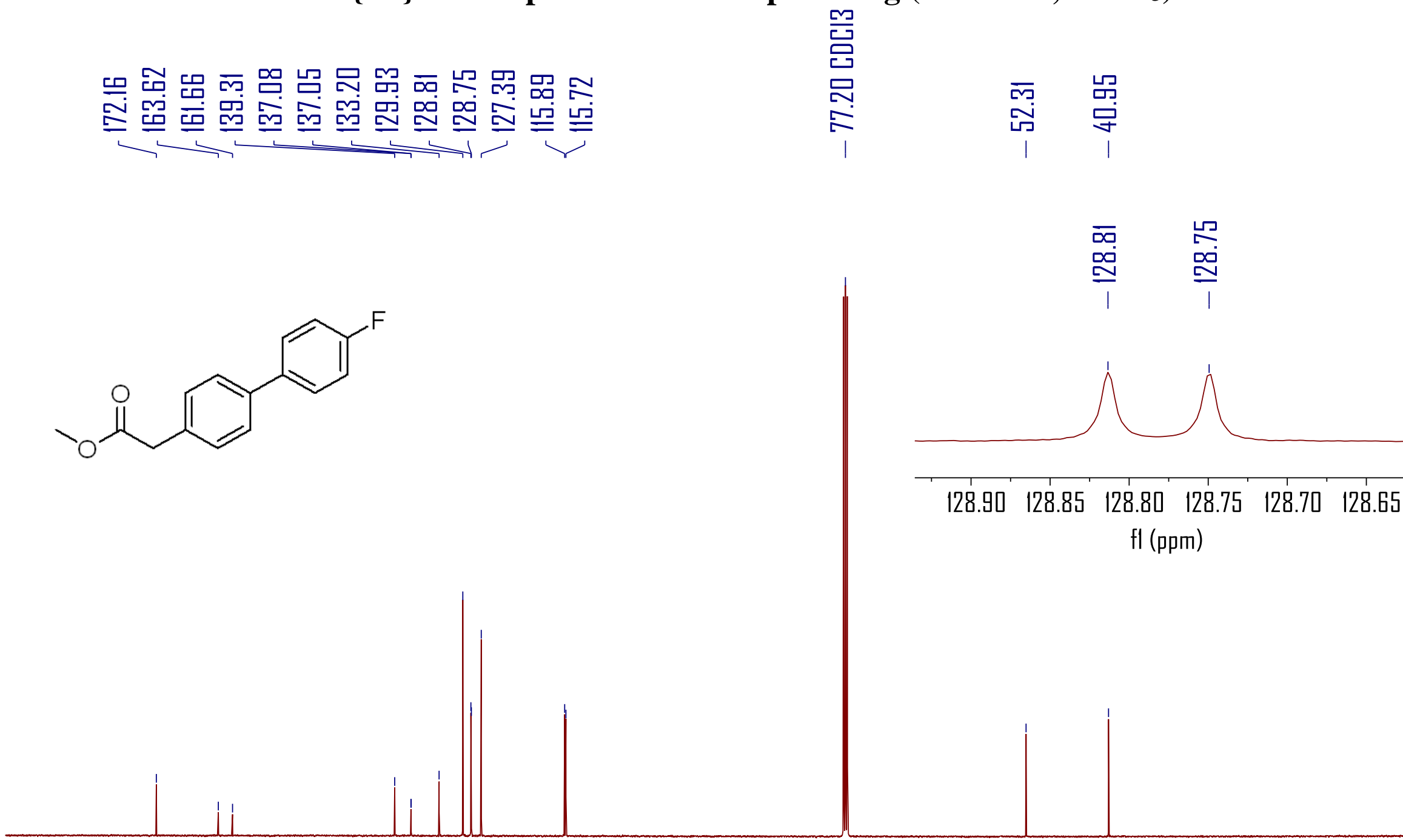

$\begin{array}{llllll}128.90 & 128.85 & 128.80 & 128.75 & 128.70 & 128.65\end{array}$

$$
\mathrm{fl}(\mathrm{ppm})
$$

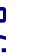

1

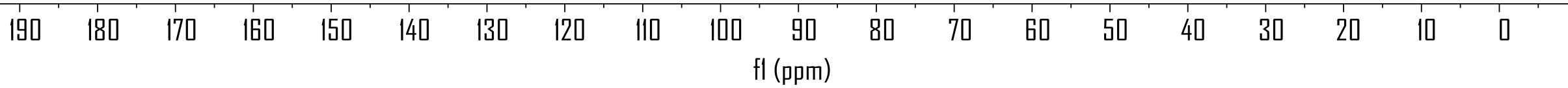




\section{${ }^{19} \mathrm{~F}\left\{{ }^{1} \mathrm{H}\right\}$ NMR spectrum for compound $3 \mathrm{~g}\left(376 \mathrm{MHz}, \mathrm{CDCl}_{3}\right)$}

过
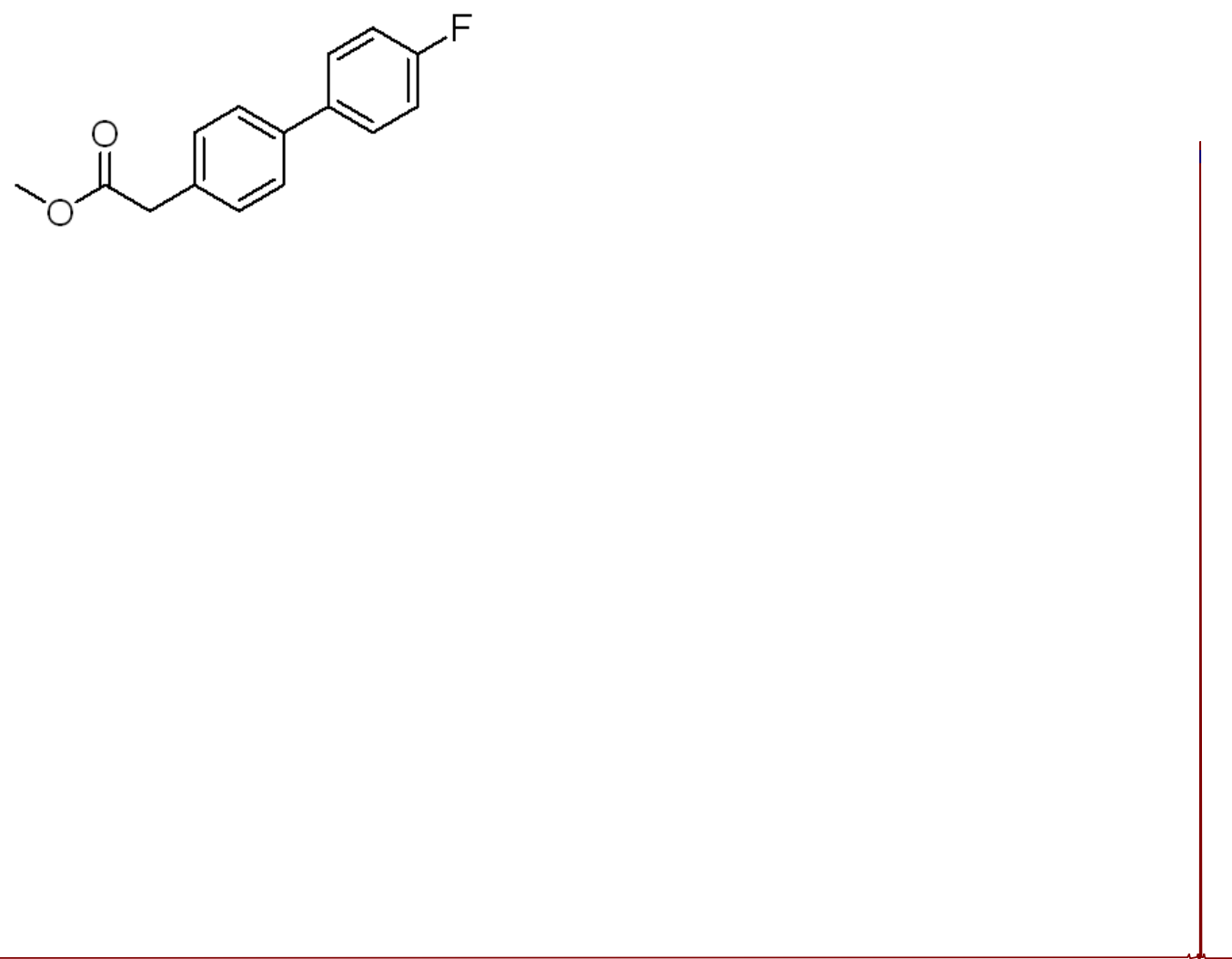

$\begin{array}{lllllllllllllllllllllllll}10 & 0 & -10 & -20 & -30 & -40 & -50 & -60 & -70 & -80 & -90 & -100 & -110 & -120 & -130 & -140 & -150 & -160 & -170 & -180 & -190 & -200 & -210\end{array}$ 


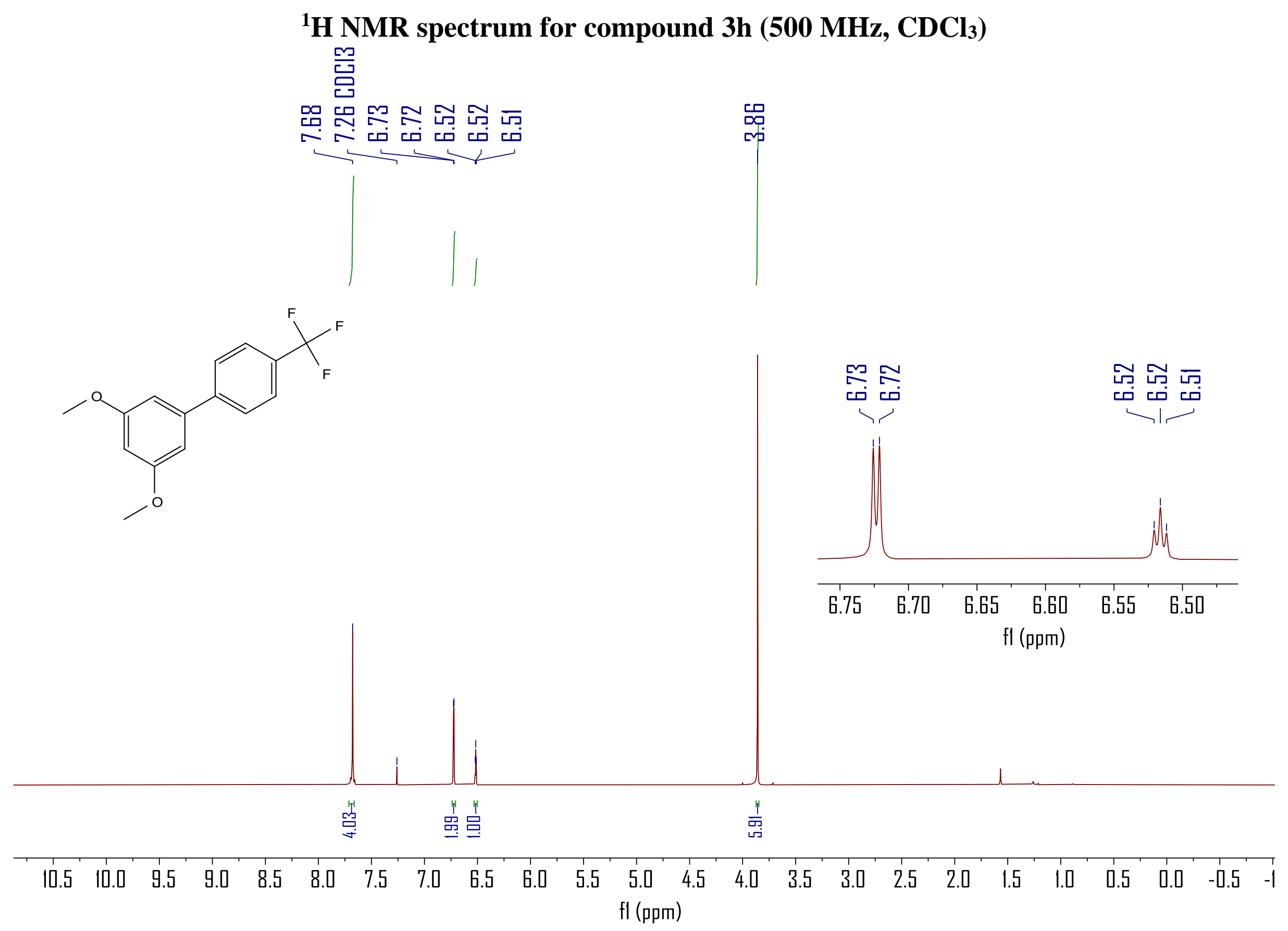




\section{${ }^{13} \mathrm{C}\left\{{ }^{1} \mathrm{H}\right\}$ NMR spectrum for compound $3 \mathrm{~h}\left(125 \mathrm{MHz}, \mathrm{CDCl}_{3}\right)$}

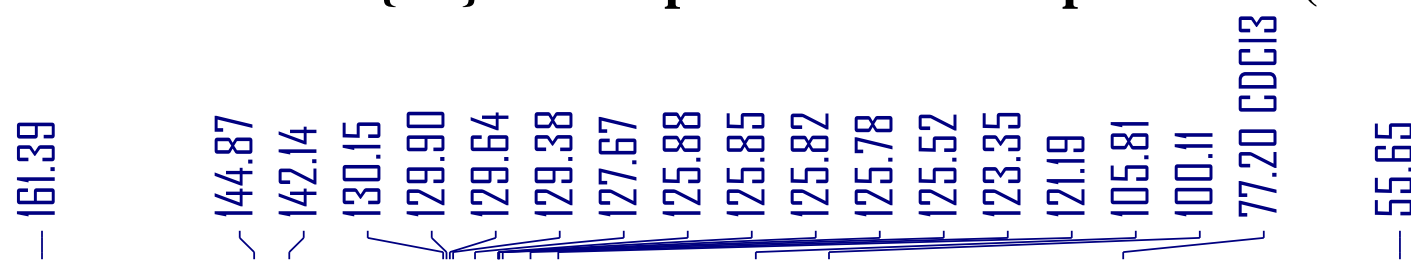

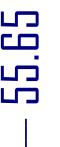

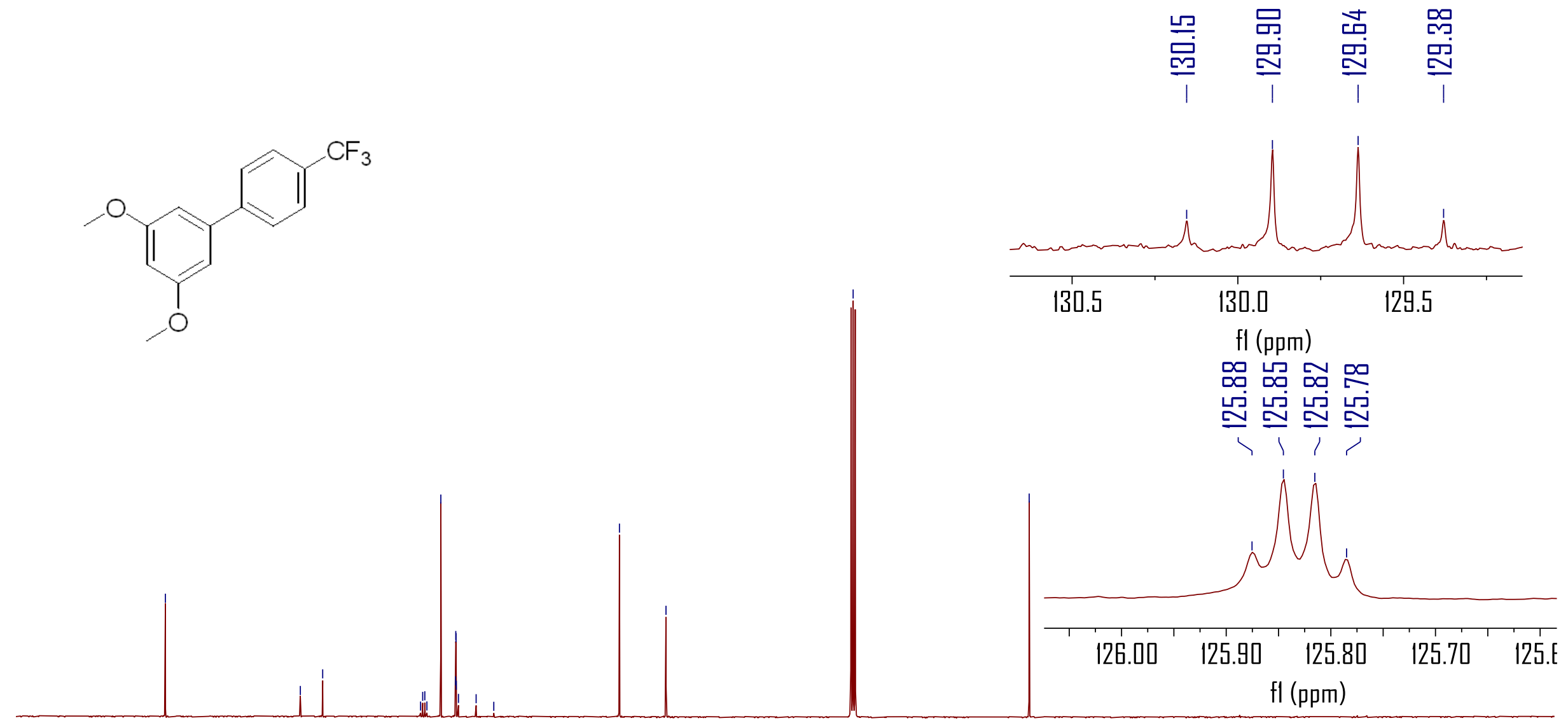

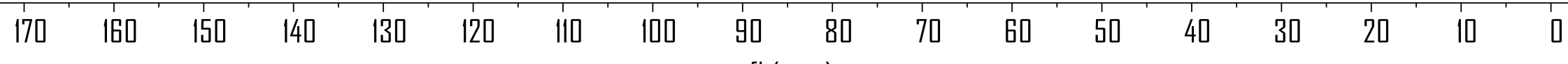

$\mathrm{fl}(\mathrm{ppm})$ 


\section{${ }^{19} \mathrm{~F}\left\{{ }^{1} \mathrm{H}\right\} \mathrm{NMR}$ spectrum for compound $3 \mathrm{~h}\left(376 \mathrm{M}, \mathrm{CDCl}_{3}\right)$}

藏

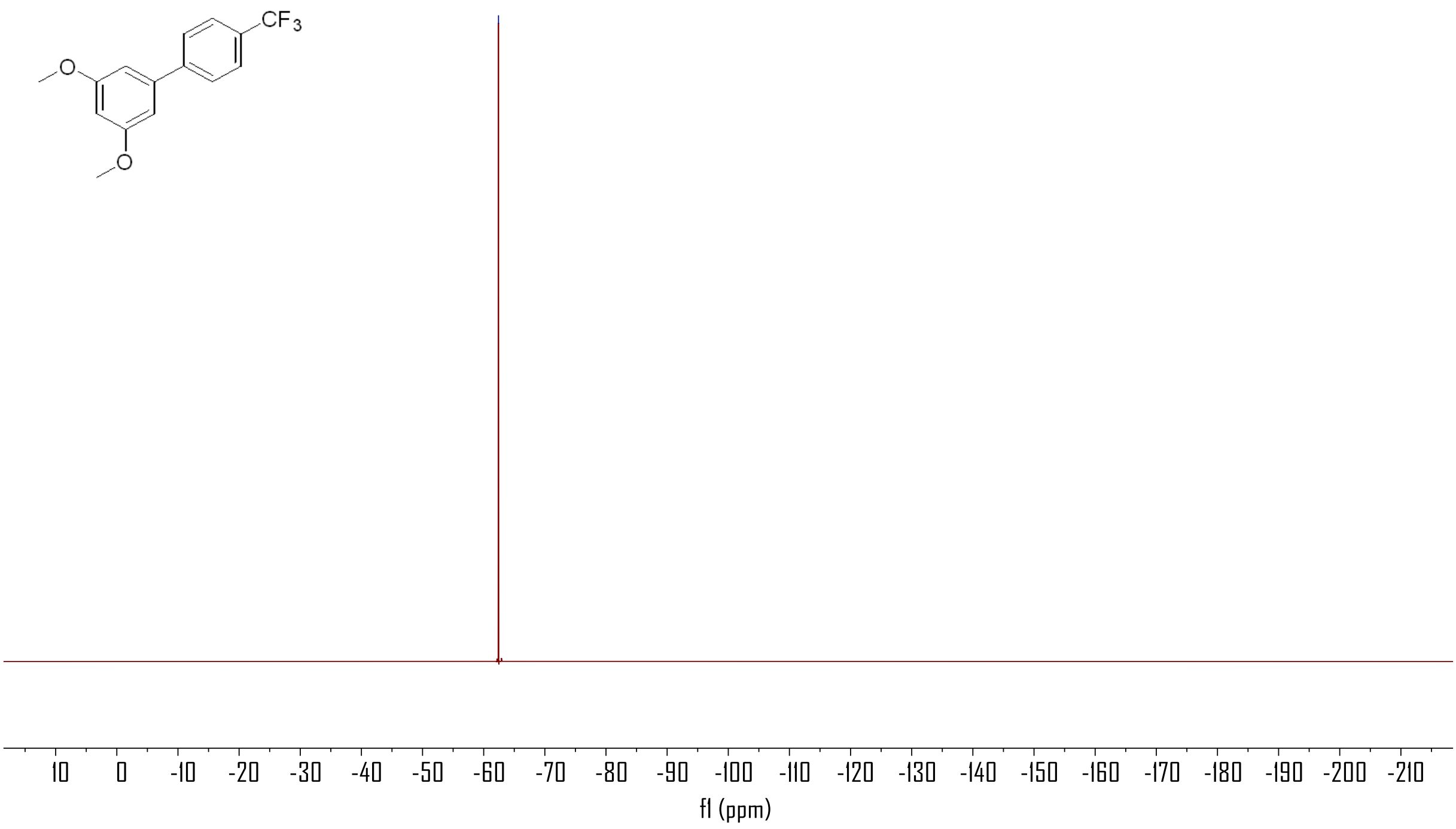




\section{${ }^{1} \mathrm{H}$ NMR spectrum for compound $3 \mathrm{i}\left(500 \mathrm{MHz}, \mathrm{C}_{6} \mathrm{D}_{6}\right)$}

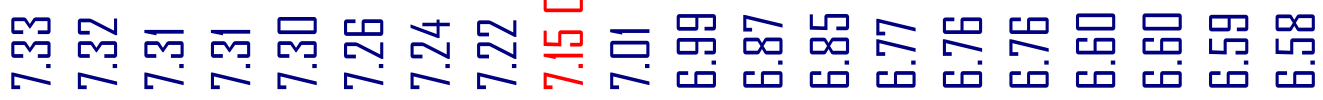
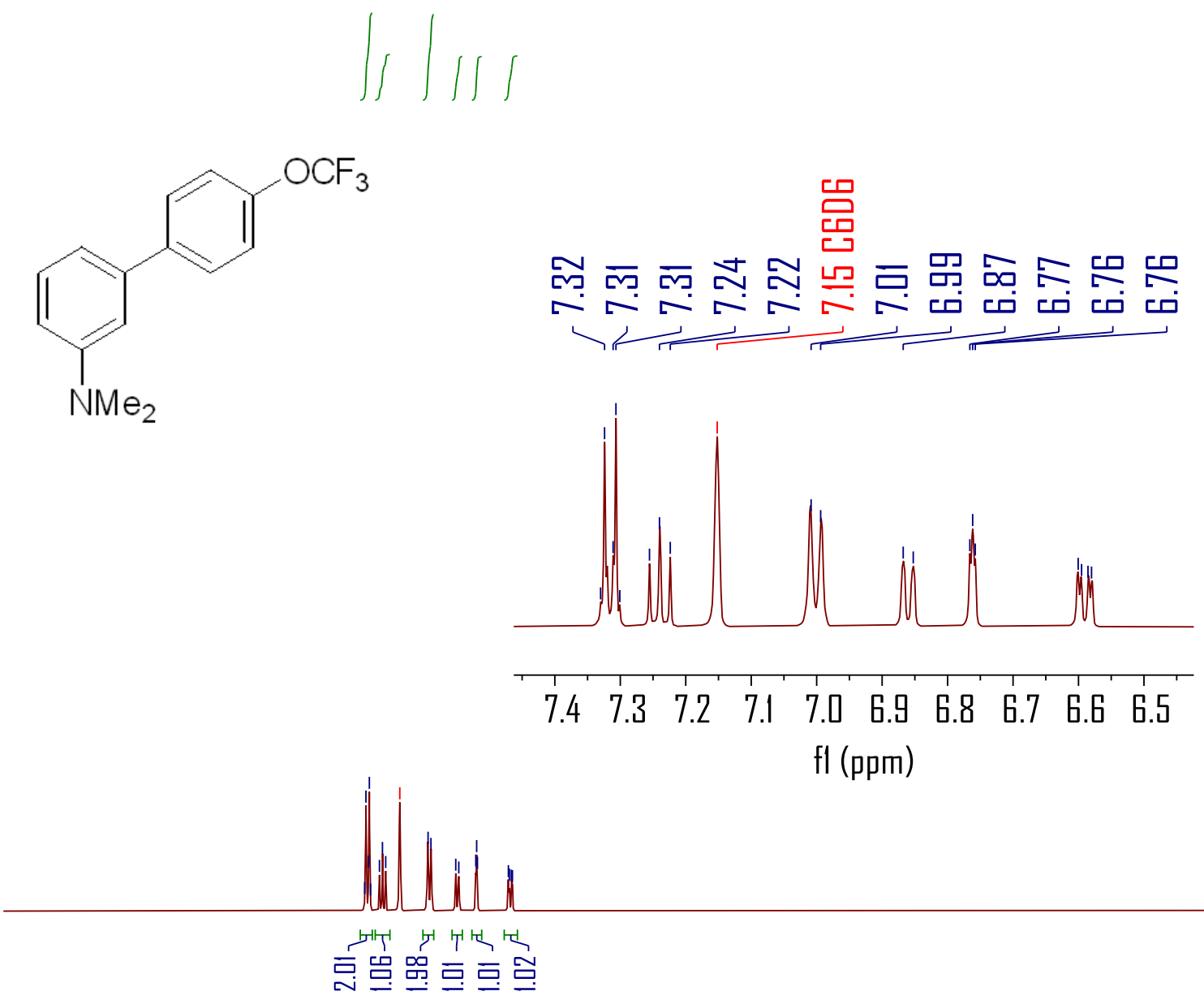

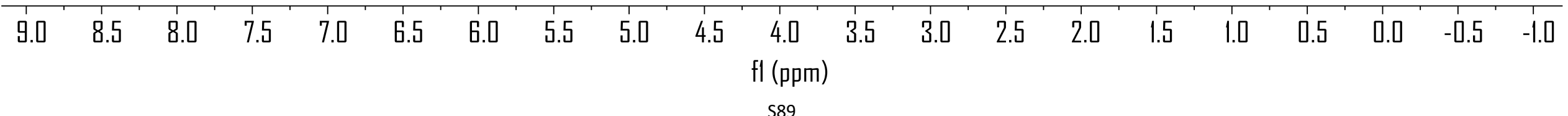




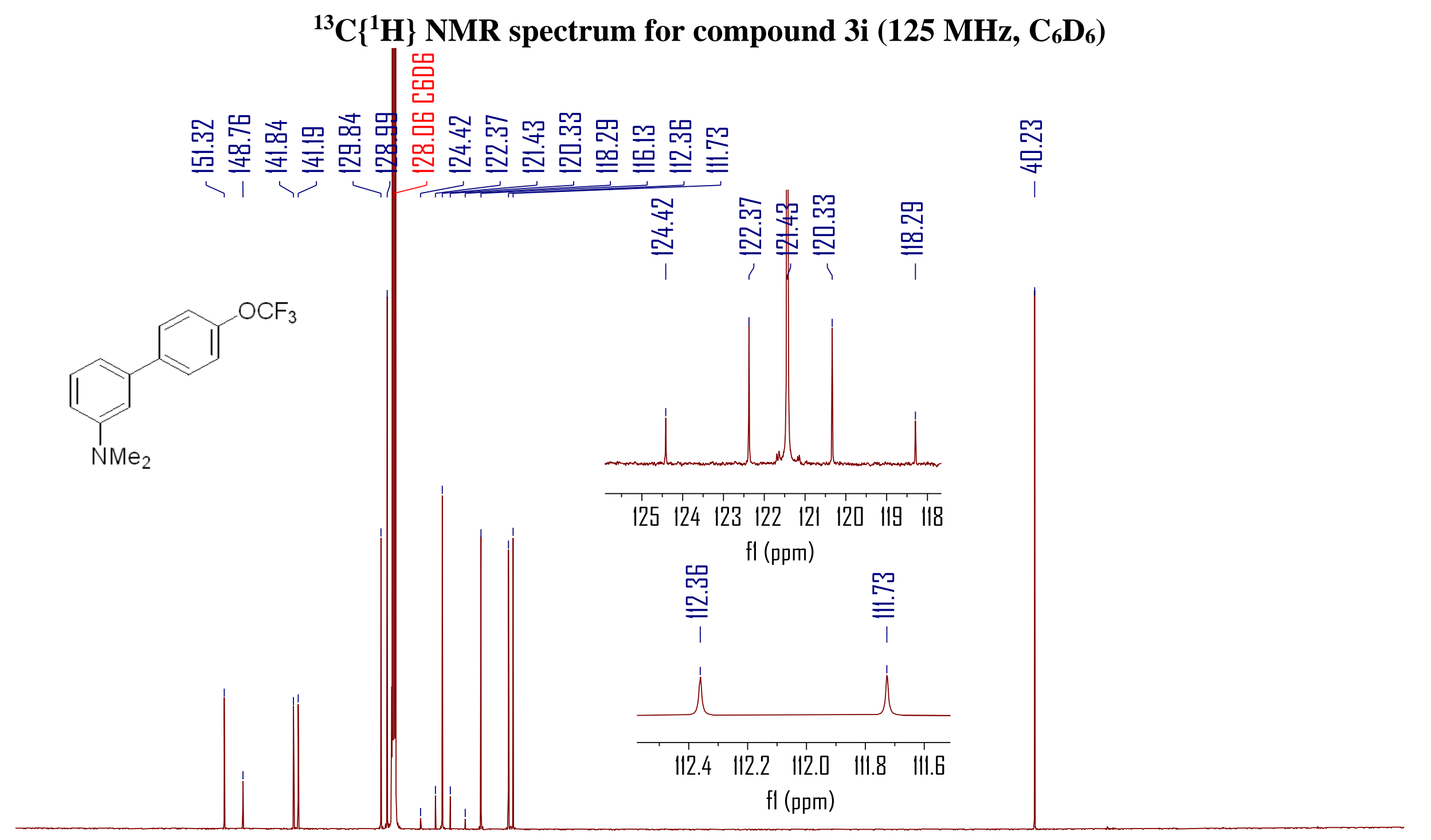

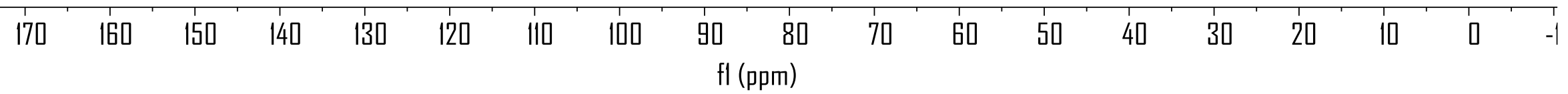




\section{${ }^{19} \mathrm{~F}\left\{{ }^{1} \mathrm{H}\right\}$ NMR spectrum for compound $3 \mathrm{i}\left(376 \mathrm{MHz}, \mathrm{C}_{6} \mathrm{D}_{6}\right)$}

牙
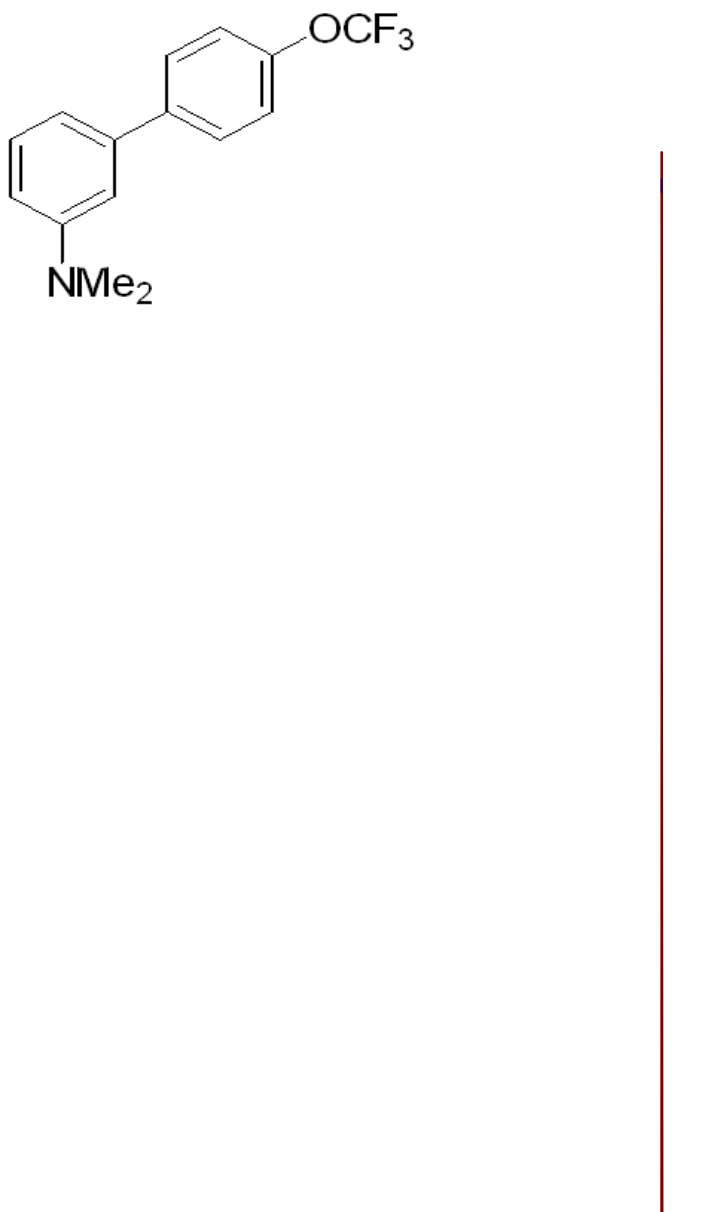

$\begin{array}{lllllllllll}10 & 0 & -10 & -20 & -30 & -40 & -50 & -60 & -70 & -80 & -90\end{array}$

$\begin{array}{lllllllllllll}-90 & -100 & -110 & -120 & -130 & -140 & -150 & -160 & -170 & -180 & -190 & -200 & -210\end{array}$ $\mathrm{fl}(\mathrm{ppm})$ 


\section{${ }^{1} \mathrm{H}$ NMR spectrum for compound $3 \mathrm{j}$ (500 $\left.\mathrm{MHz}, \mathrm{CDCl}_{3}\right)$}

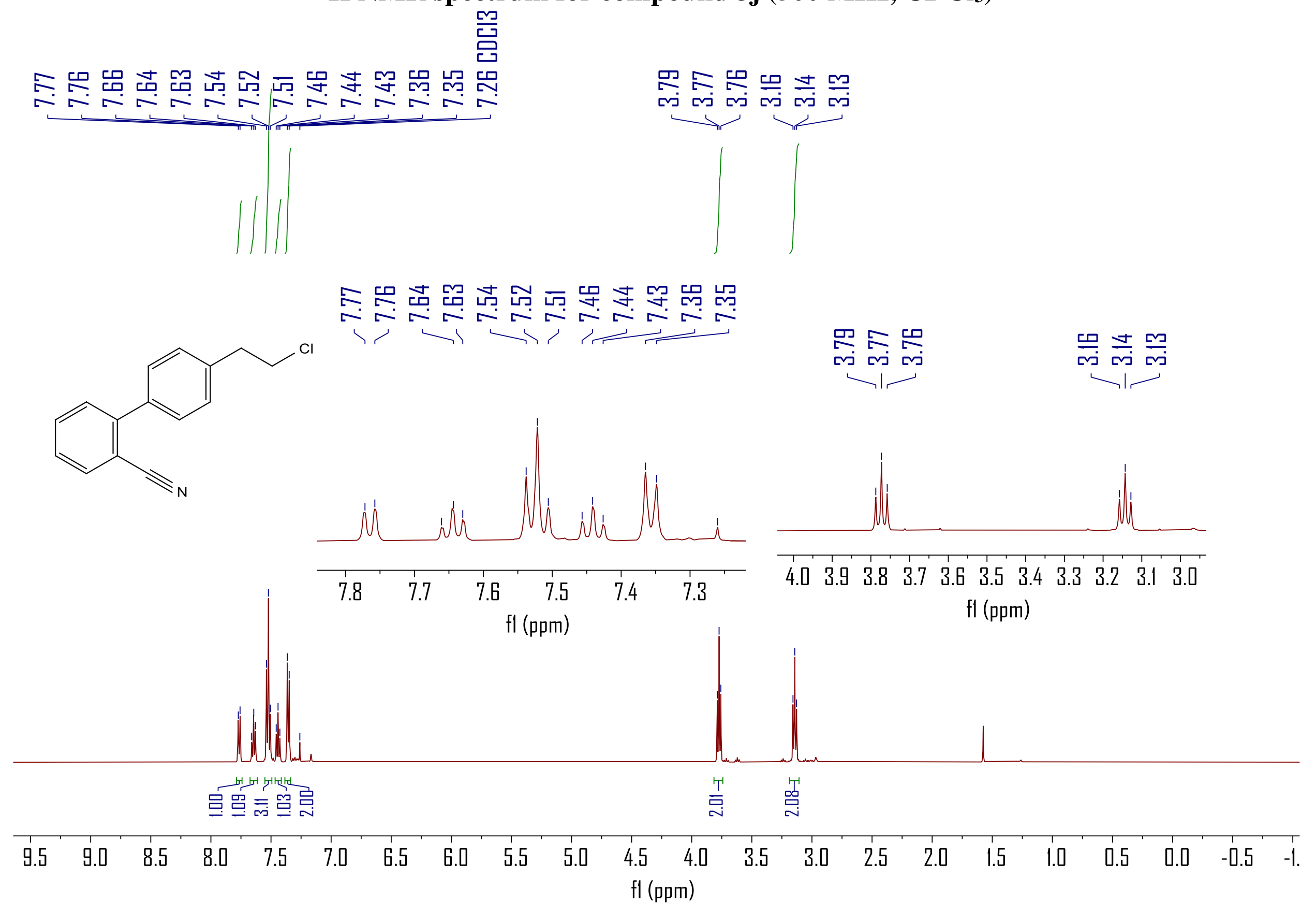




\section{${ }^{13} \mathrm{C}\left\{{ }^{1} \mathrm{H}\right\}$ NMR spectrum for compound $3 \mathrm{j}\left(125 \mathrm{MHz}, \mathrm{CDCl}_{3}\right)$}

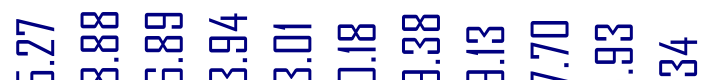

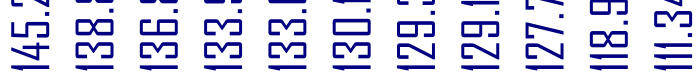
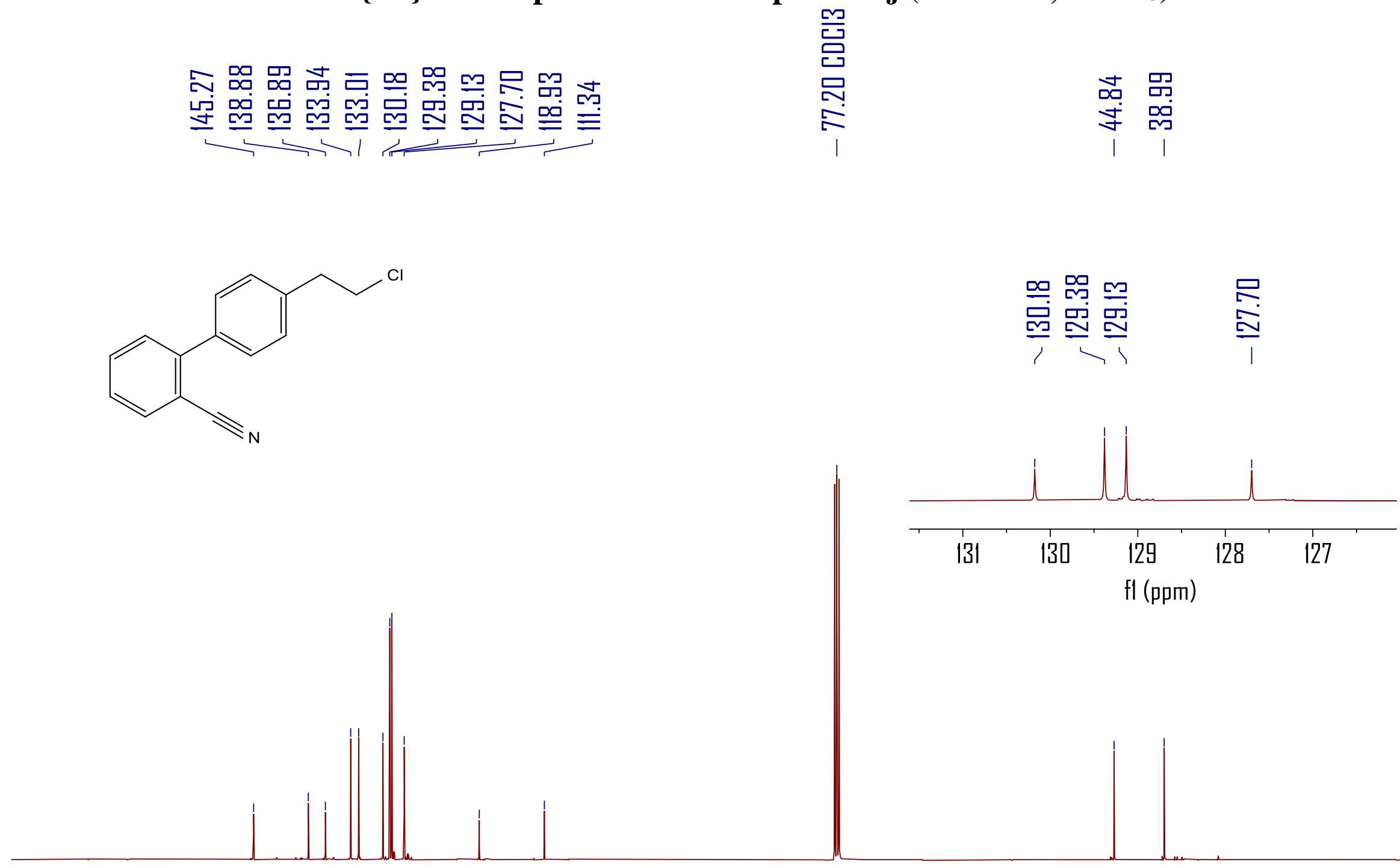

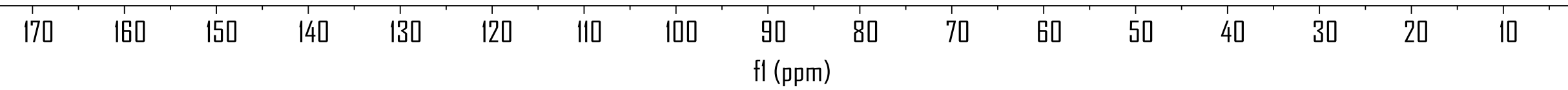


${ }^{1} \mathrm{H}$ NMR spectrum for compound 3k (500 $\mathrm{MHz}^{\mathrm{CDCl}} \mathrm{CD}_{3}$

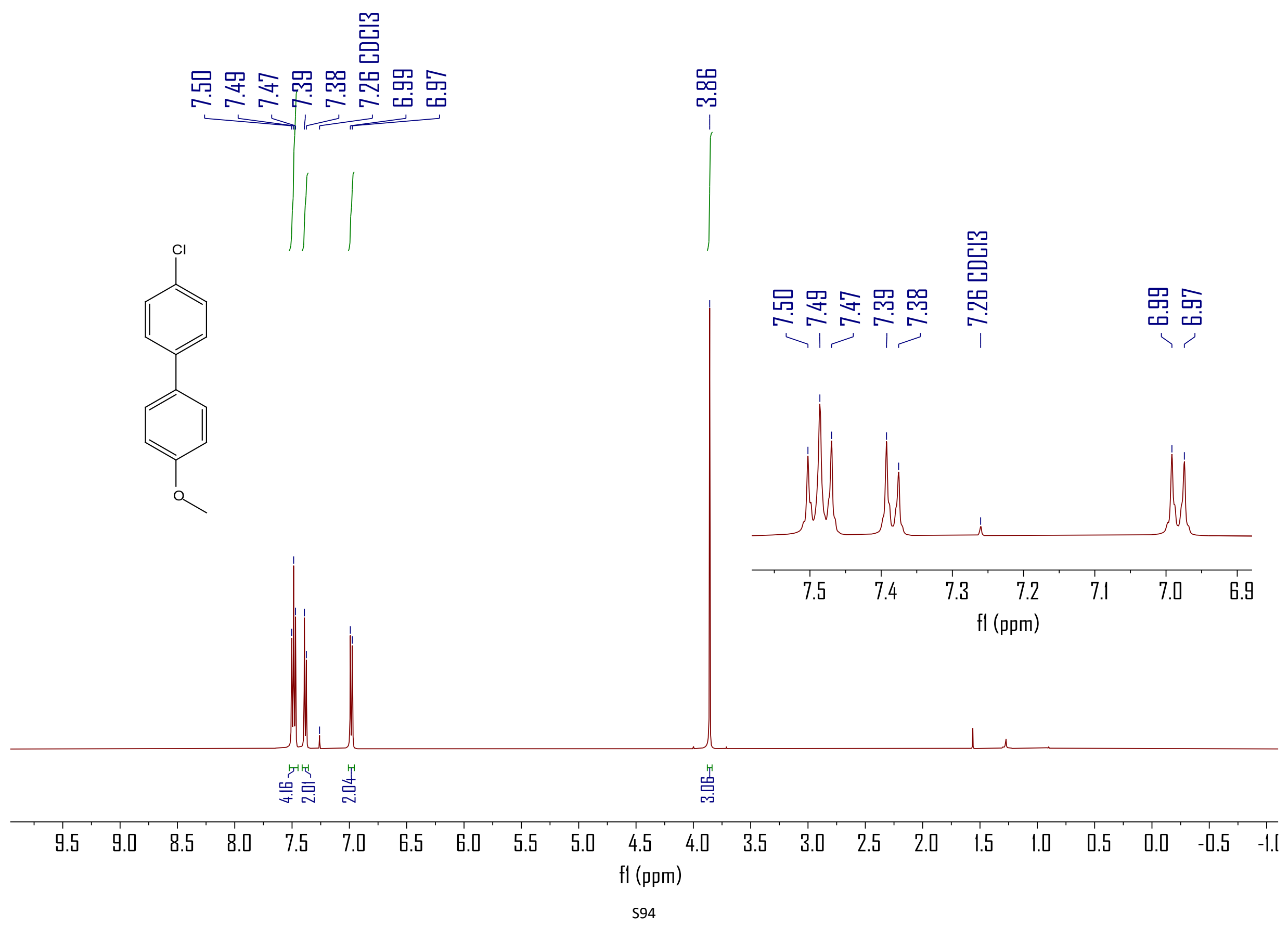




\section{${ }^{13} \mathrm{C}$ NMR spectrum for compound 3k (125 $\left.\mathrm{MHz}, \mathrm{CDCl}_{3}\right)$}

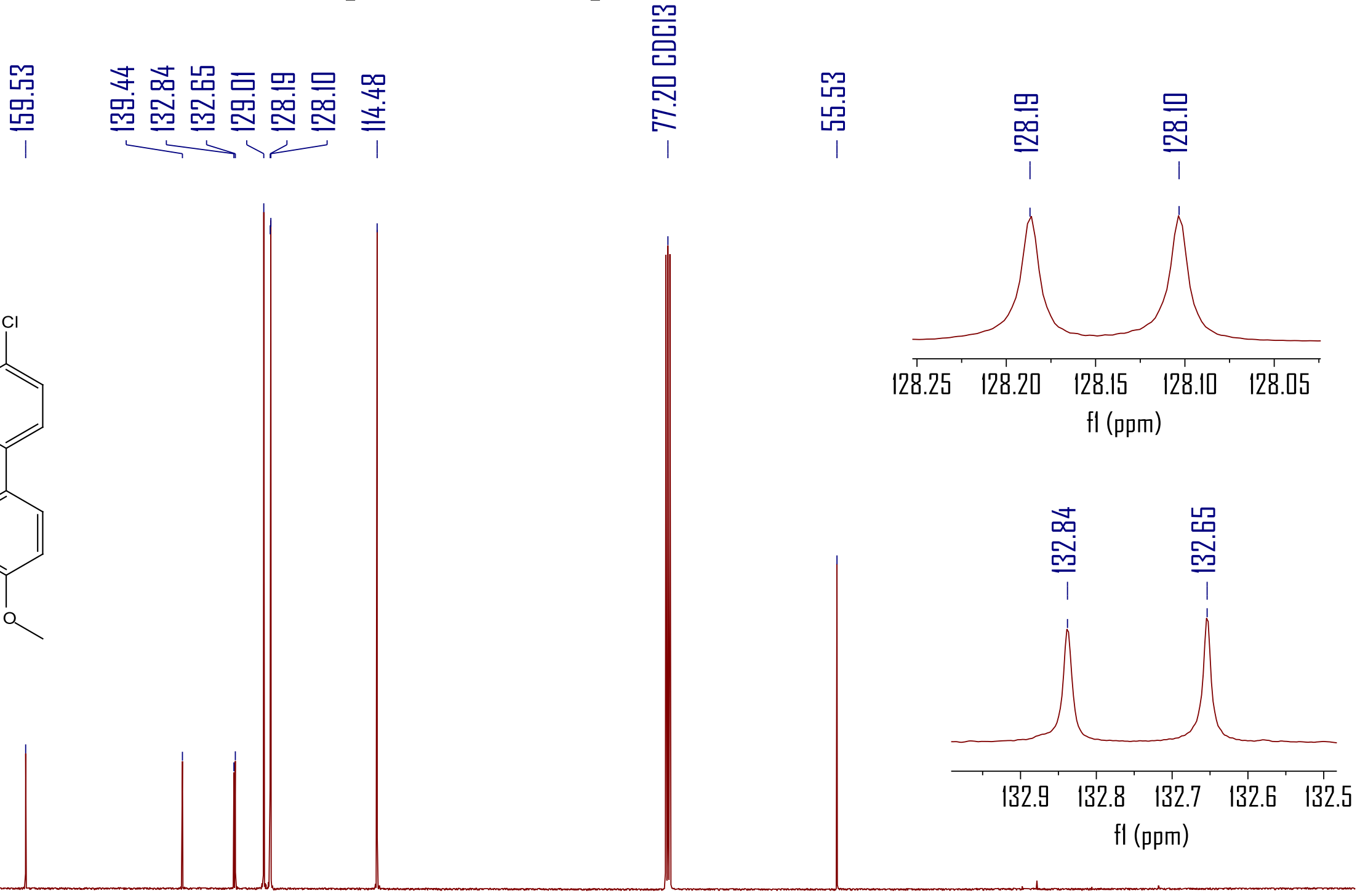

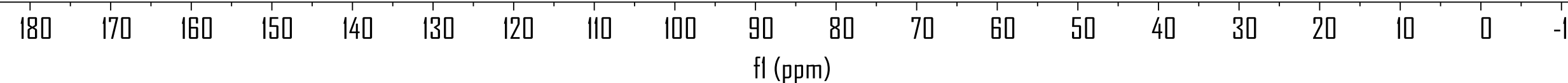




\section{${ }^{1} \mathrm{H}$ NMR spectrum for compound $31\left(500 \mathrm{MHz}, \mathrm{CDCl}_{3}\right)$}

管

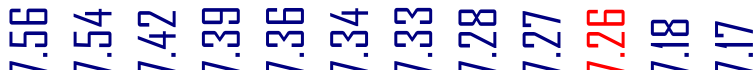

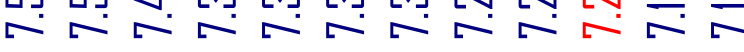
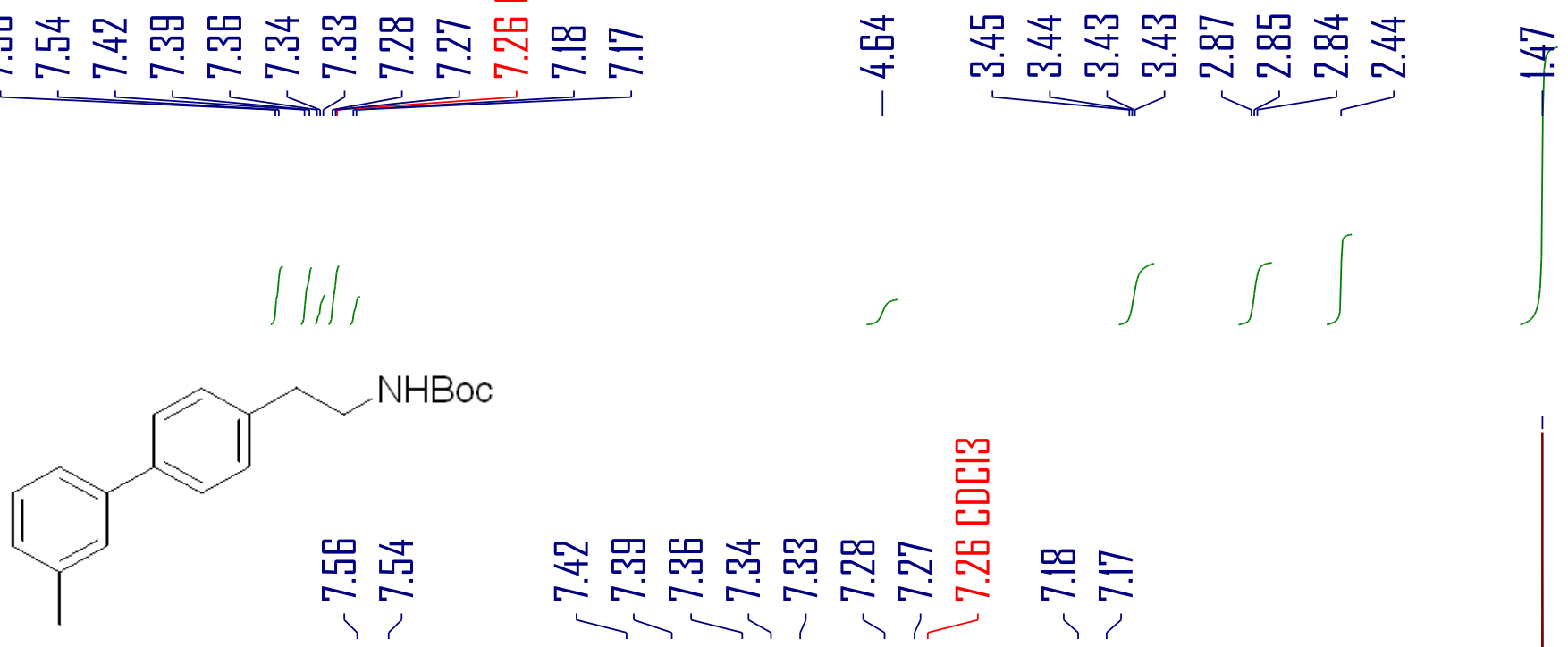

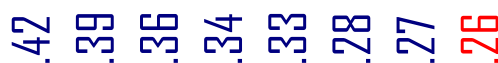

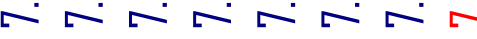

믄 둔
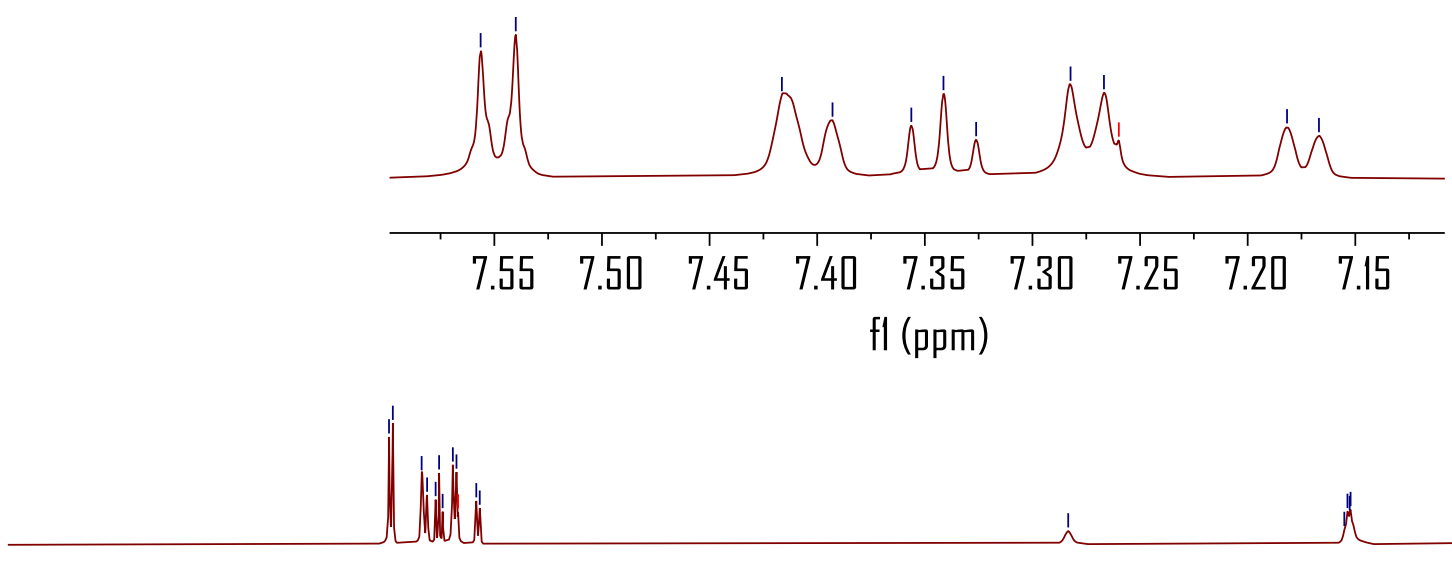

喿哭总哥产

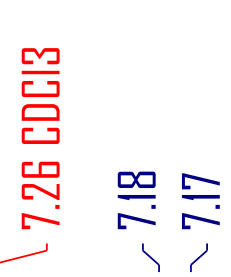

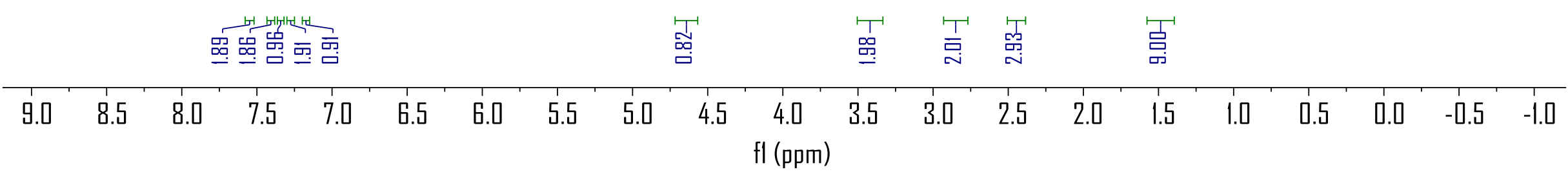

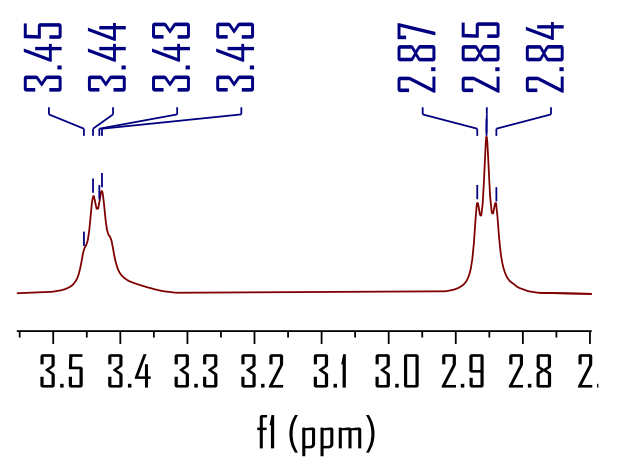

$\mathrm{fl}(\mathrm{ppm})$ 


\section{${ }^{13} \mathrm{C}\left\{{ }^{1} \mathrm{H}\right\}$ NMR spectrum for compound 31 (125 $\left.\mathrm{MHz}^{\mathrm{CDCl}} \mathrm{CD}_{3}\right)$}

\begin{tabular}{|c|c|c|}
\hline 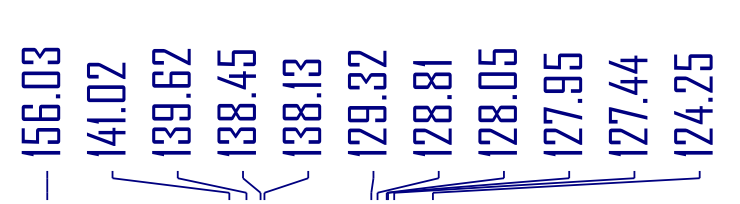 & 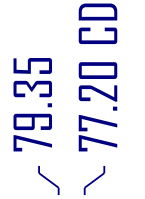 & 虽忽品品品 \\
\hline
\end{tabular}

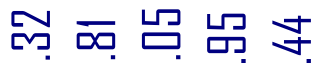

回 吅㗁豆

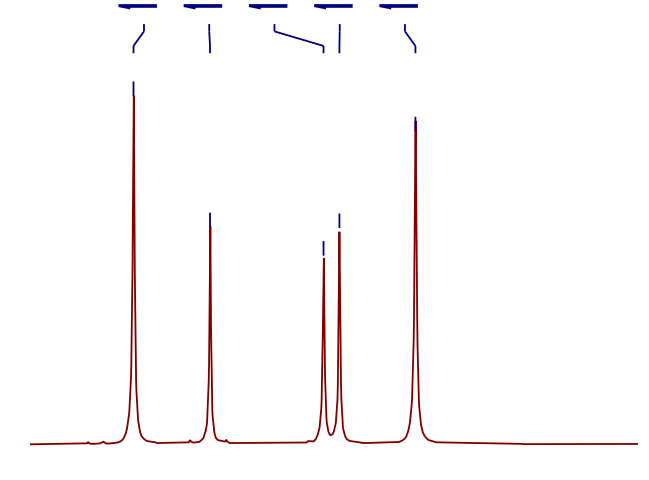

130

129

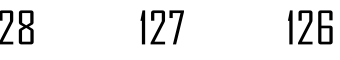

fl (ppm)

$190 \quad 180 \quad 17$

$160 \quad 150 \quad 140$

130

120

$110 \quad 100$

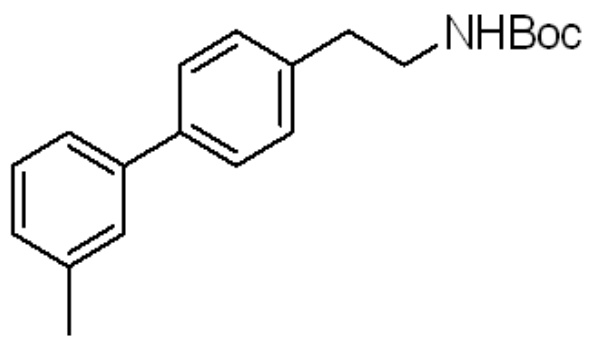

$138.8 \quad 138.4 \quad 138.0$

$\mathrm{fl}$ (ppm)
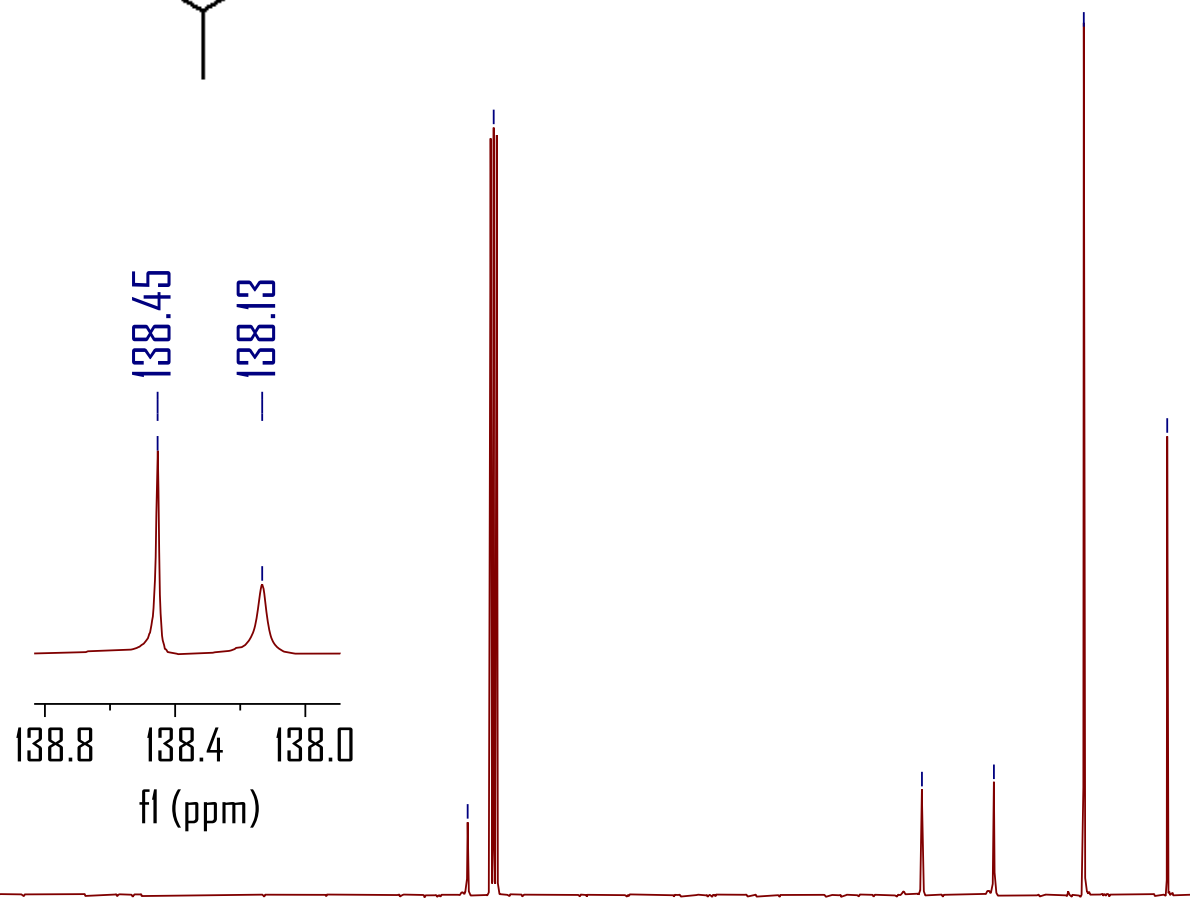

$\mathrm{fl}$ (ppm) 


\section{${ }^{1} \mathrm{H}$ NMR spectrum for compound $3 \mathrm{~m}\left(500 \mathrm{MHz}, \mathrm{CDCl}_{3}\right)$}

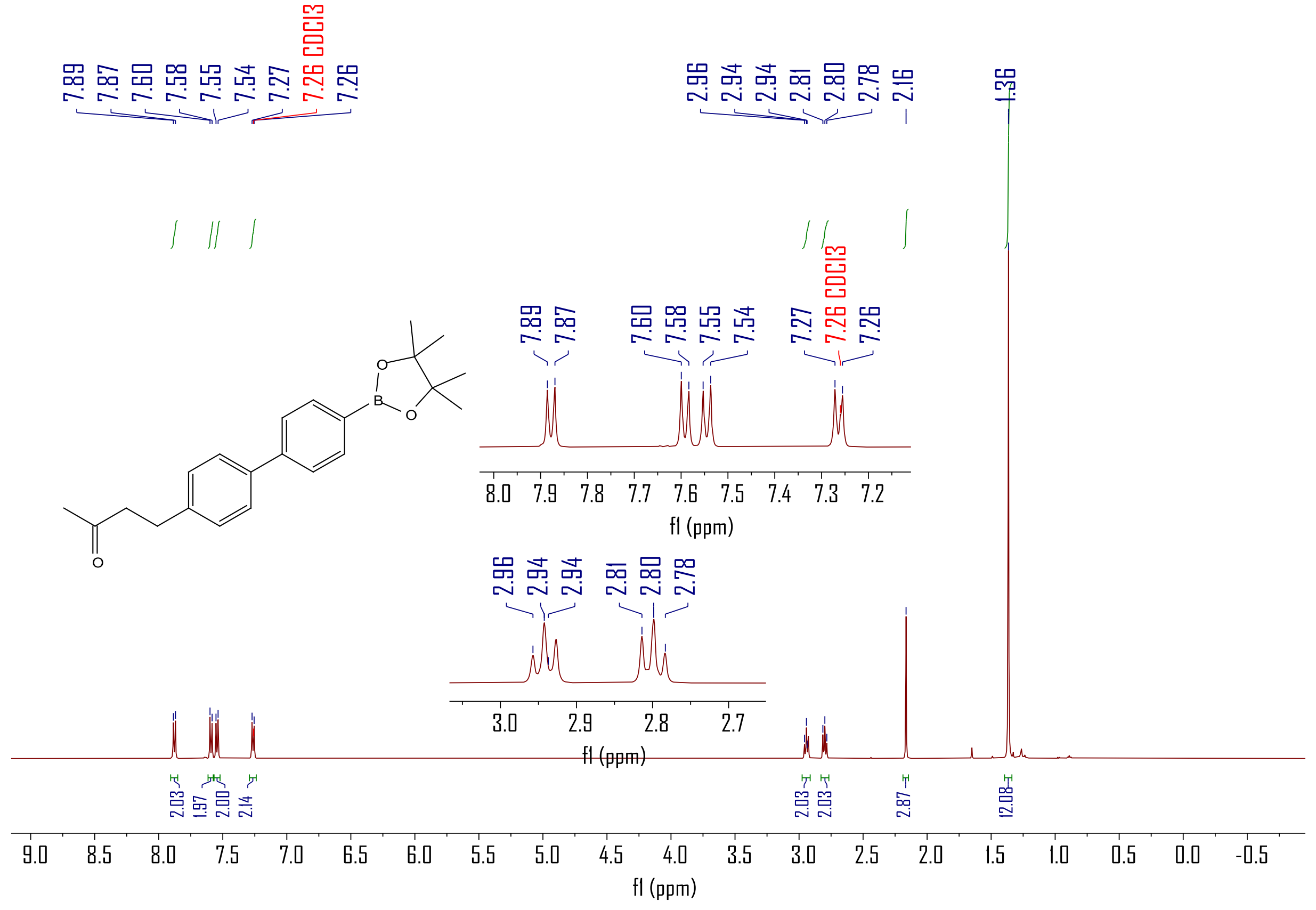




\section{${ }^{13} \mathrm{C}\left\{{ }^{1} \mathrm{H}\right\}$ NMR spectrum for compound $3 \mathrm{~m}\left(125 \mathrm{MHz}, \mathrm{CDCl}_{3}\right)$}
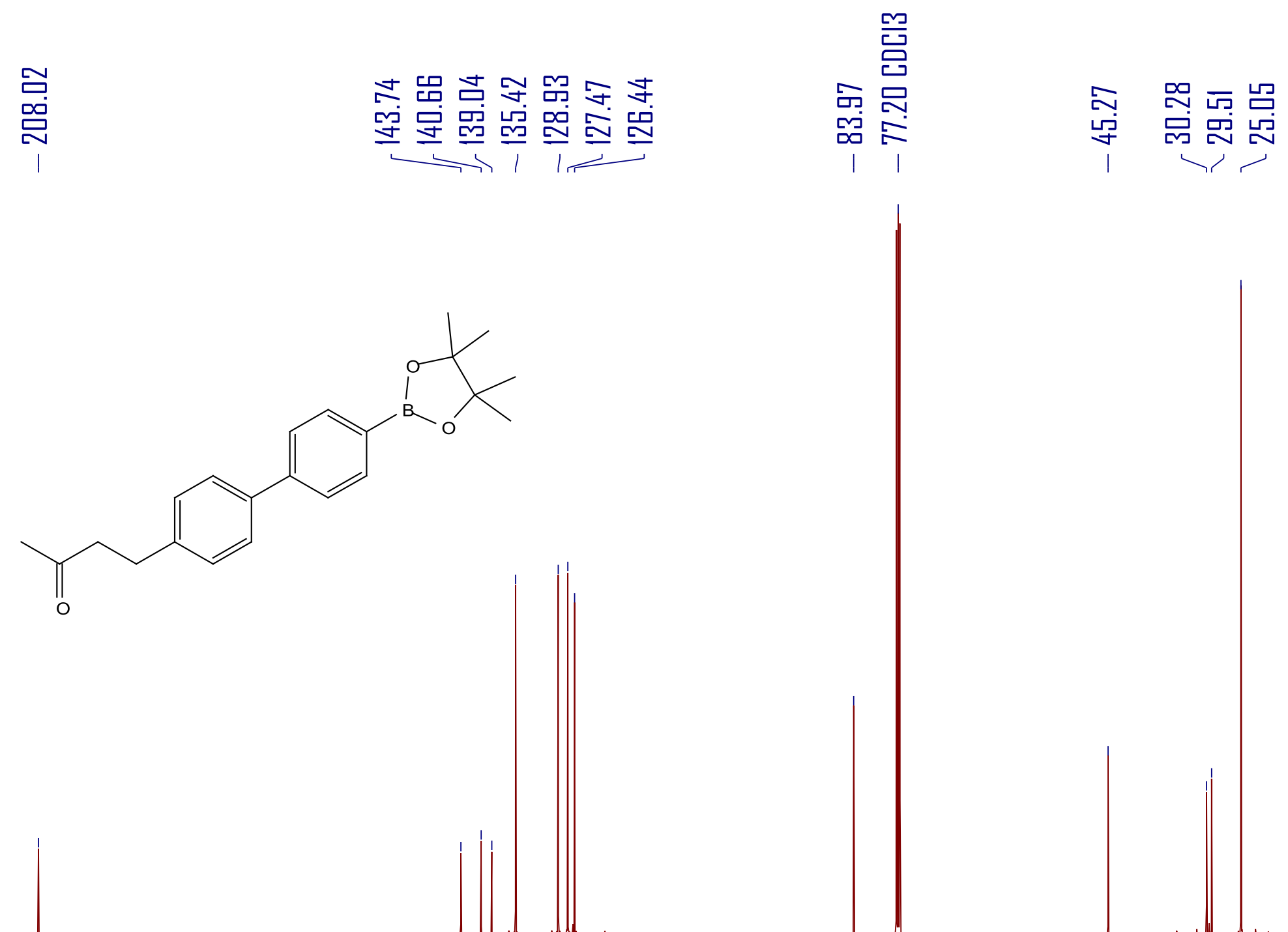

3022

$210 \quad 2$

$200 \quad 190$

$180 \quad 170$

$170 \quad 160$

$150 \quad 140$

$130 \quad 12$ fl (ppm) 
${ }^{1} \mathrm{H}$ NMR spectrum for compound 3n (500 $\left.\mathrm{MHz}, \mathrm{CDCl}_{3}\right)$

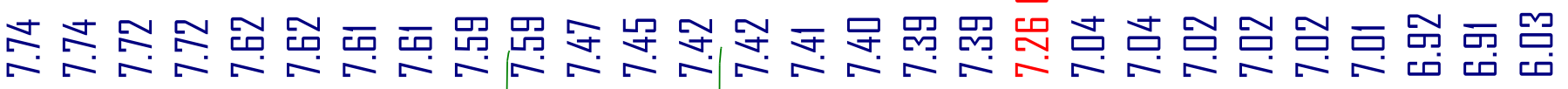

鹗
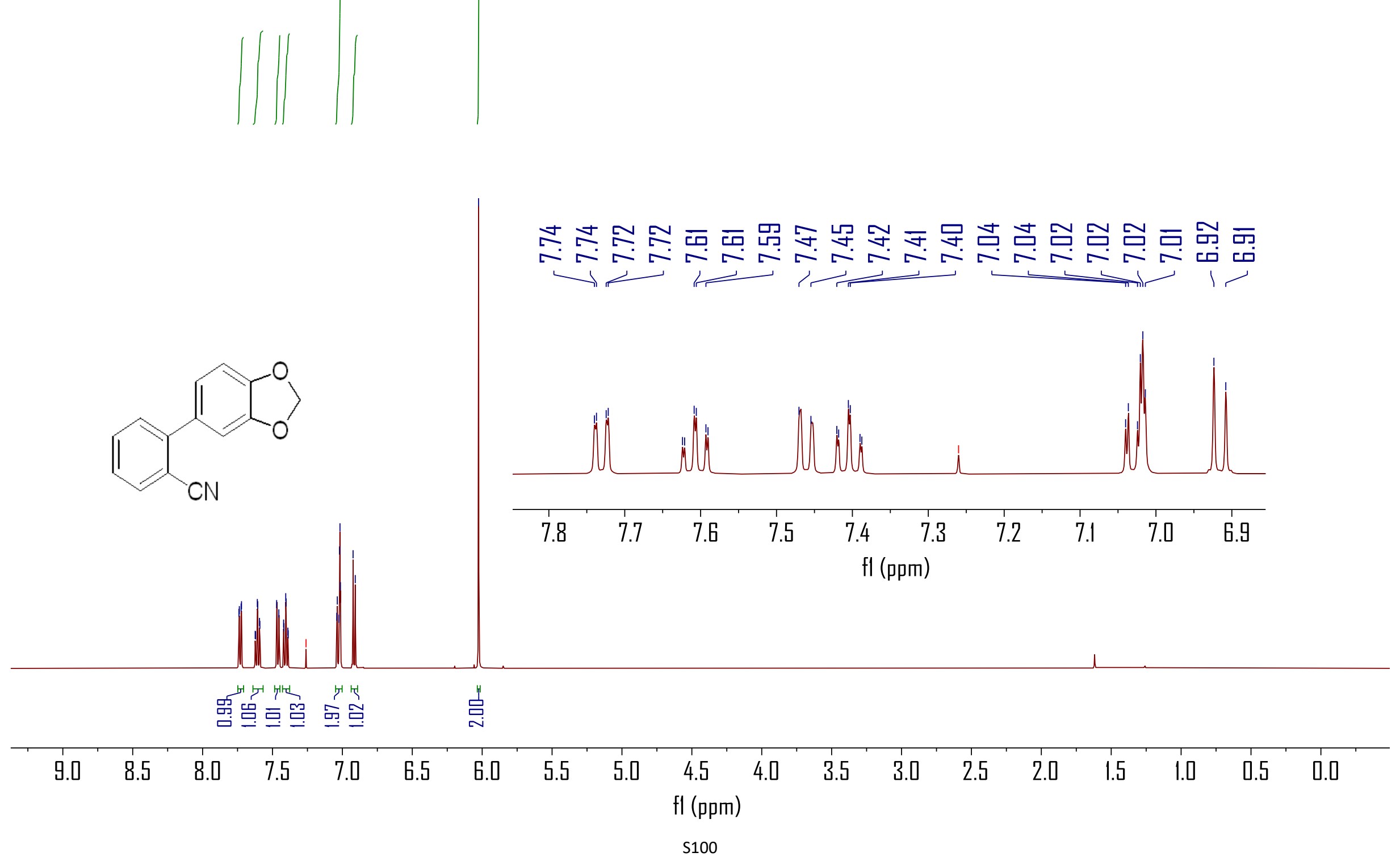


\section{${ }^{13} \mathrm{C}\left\{{ }^{1} \mathrm{H}\right\} \mathrm{NMR}$ spectrum for compound 3n (125 $\left.\mathrm{MHz}, \mathrm{CDCl}_{3}\right)$}

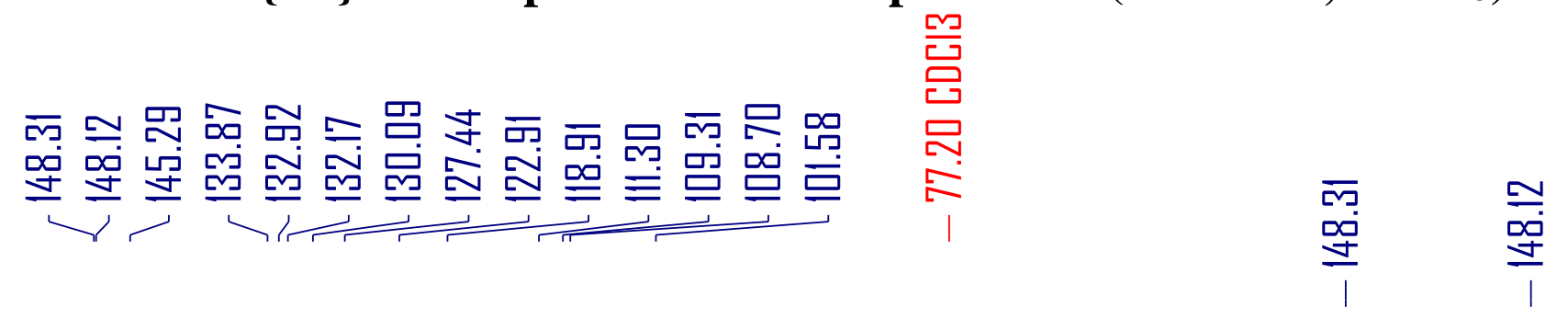
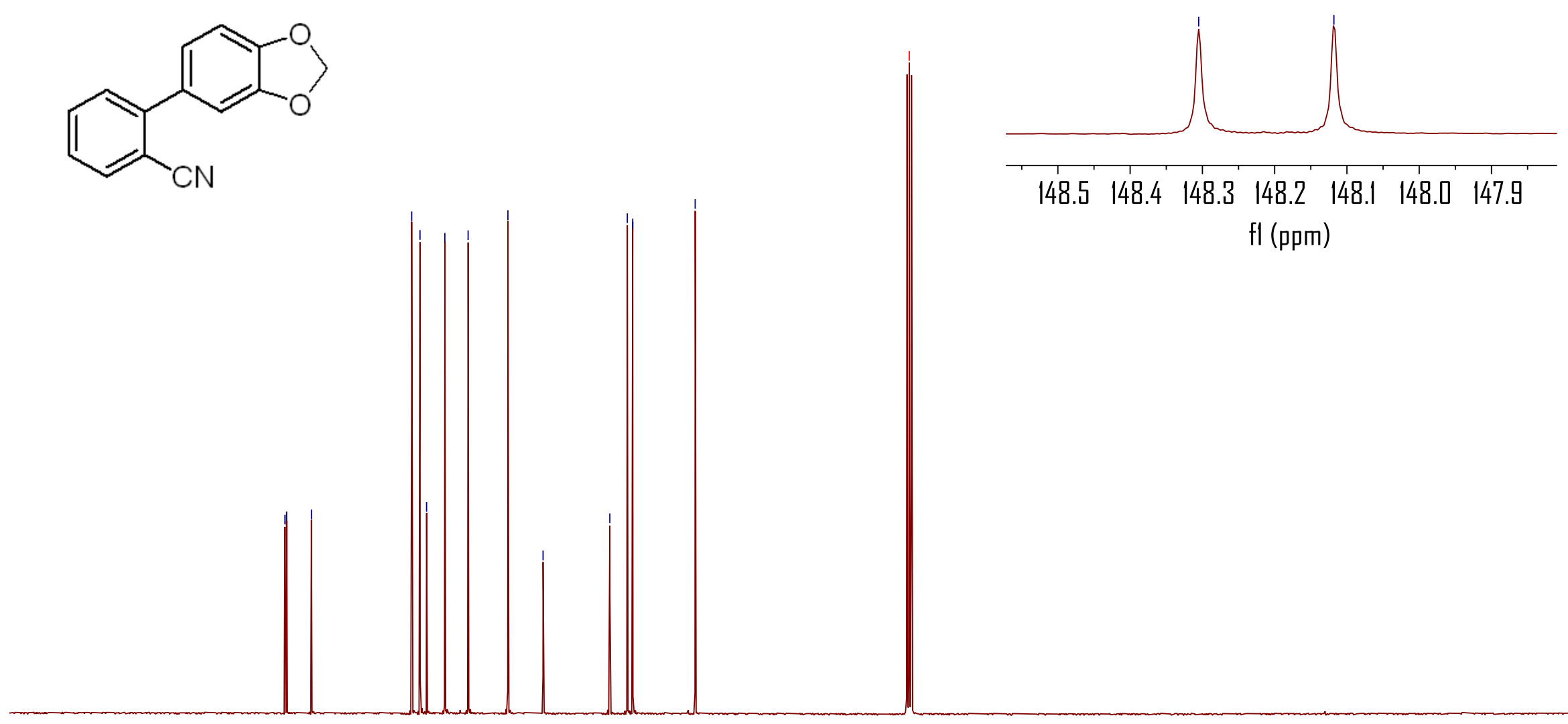

$\begin{array}{lllllll}148.5 & 148.4 & 148.3 & 148.2 & 148.1 & 148.0 & 147.9\end{array}$

$\mathrm{fl}(\mathrm{ppm})$

$\begin{array}{llllllllll}170 & 160 & 150 & 140 & 130 & 120 & 110 & 100 & 90 & 80 \\ f 1(\mathrm{ppm})\end{array}$ 


\section{${ }^{1} \mathrm{H}$ NMR spectrum for compound 30 (400 $\left.\mathrm{MHz} \mathrm{CDCl}_{3}, \mathrm{TMS}\right)$}

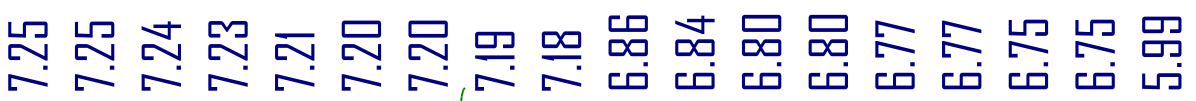

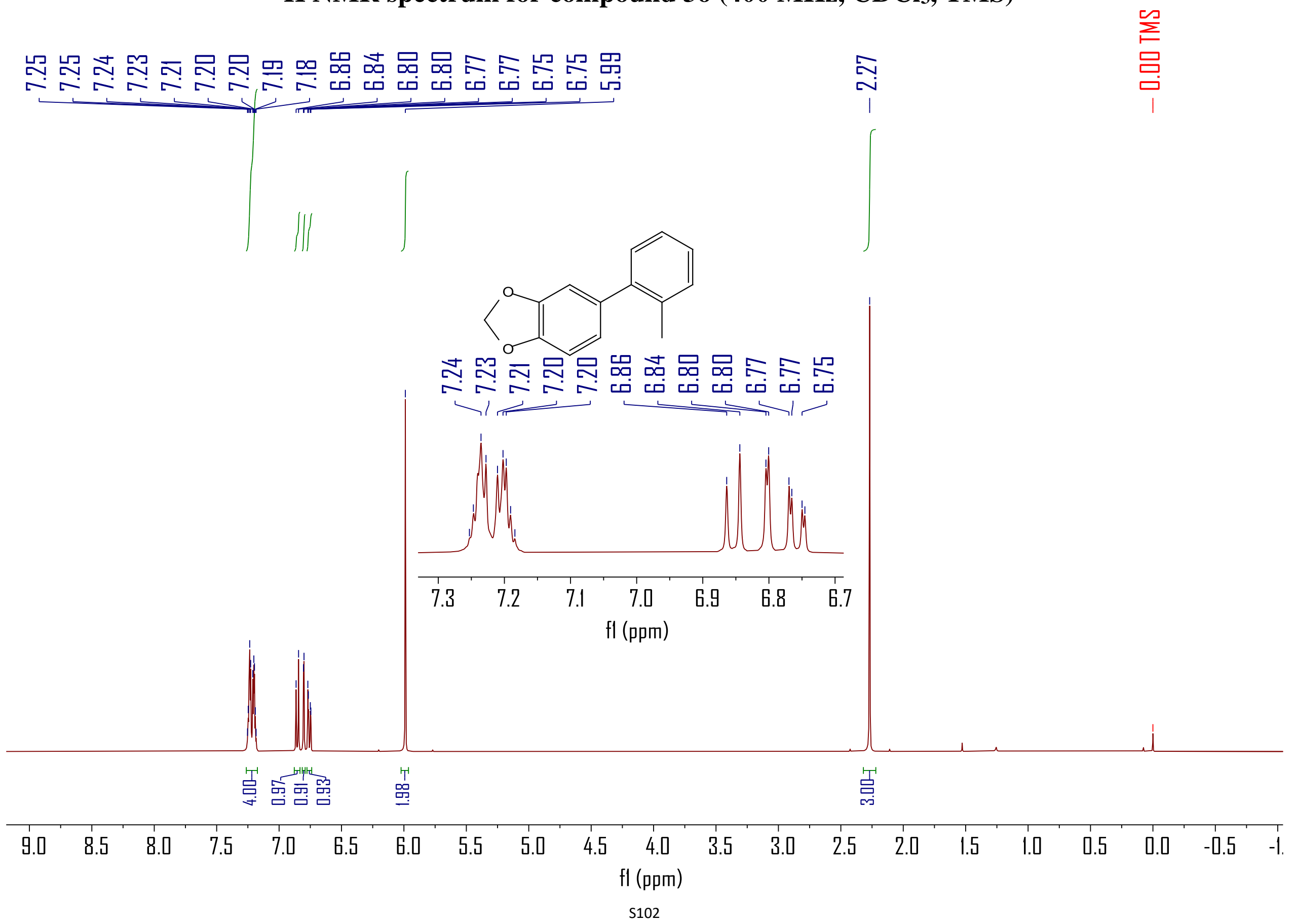




\section{${ }^{13} \mathrm{C}\left\{{ }^{1} \mathrm{H}\right\}$ NMR spectrum for compound $30\left(125 \mathrm{MHz}, \mathrm{CDCl}_{3}\right)$}

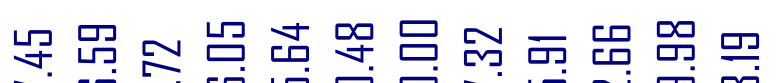

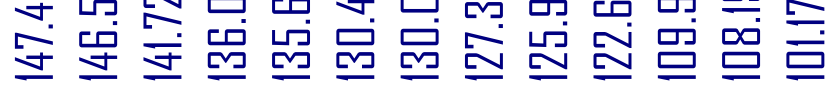

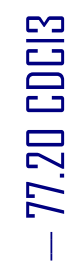

氮

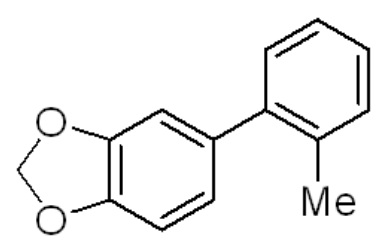

$70 \quad 60$

\begin{tabular}{lllllll}
\hline 50 & 50 & 30 & 20 & 10 & 0 & -10
\end{tabular}


${ }^{1} \mathrm{H}$ NMR spectrum for compound 3p following general procedure $\mathrm{A}\left(500 \mathrm{MHz}, \mathrm{CDCl}_{3}\right)$

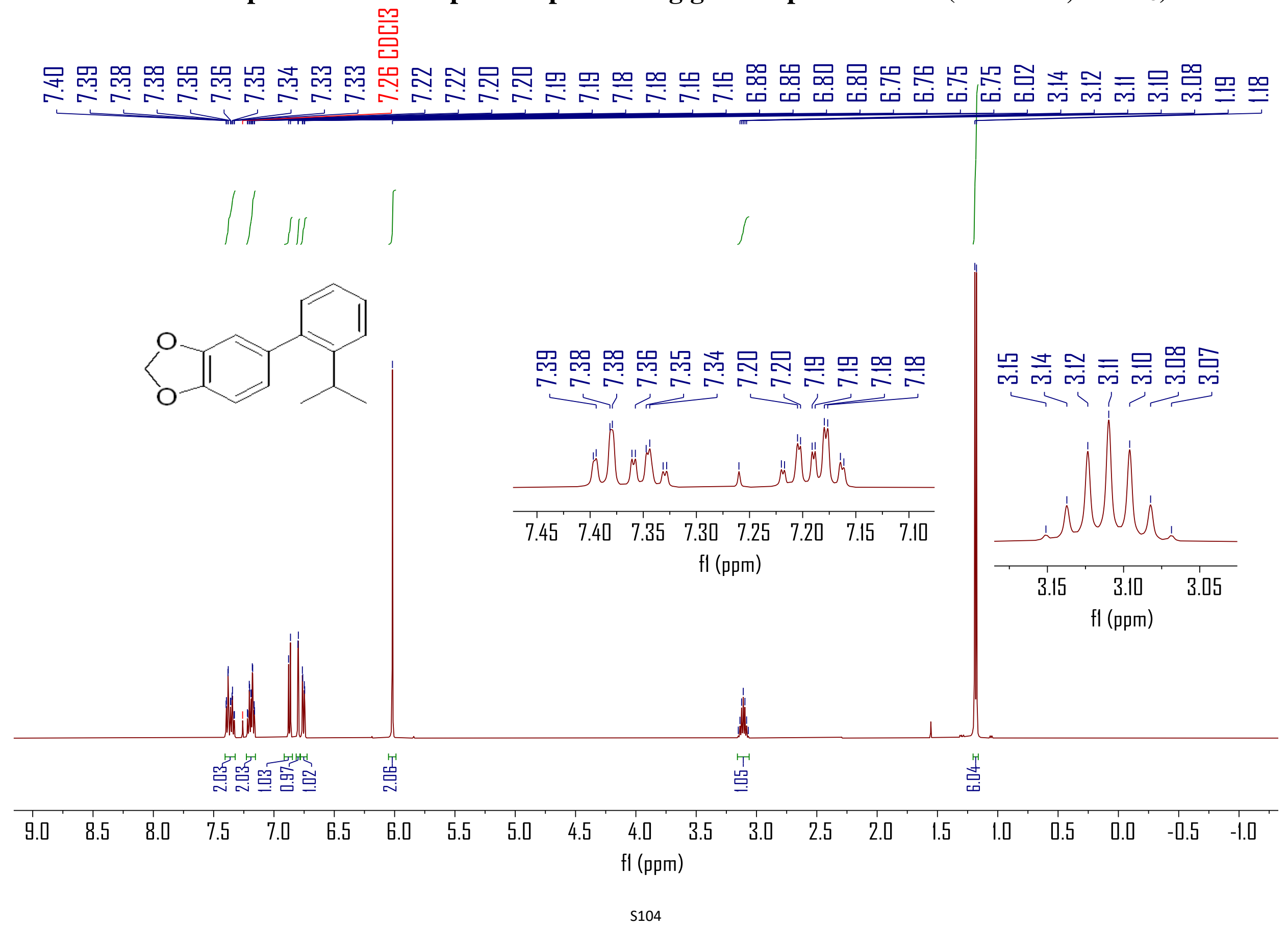




\section{${ }^{13} \mathrm{C}\left\{{ }^{1} \mathrm{H}\right\}$ NMR spectrum for compound 3p following general procedure $\mathrm{A}\left(125 \mathrm{MHz}, \mathrm{CDCl}_{3}\right)$}
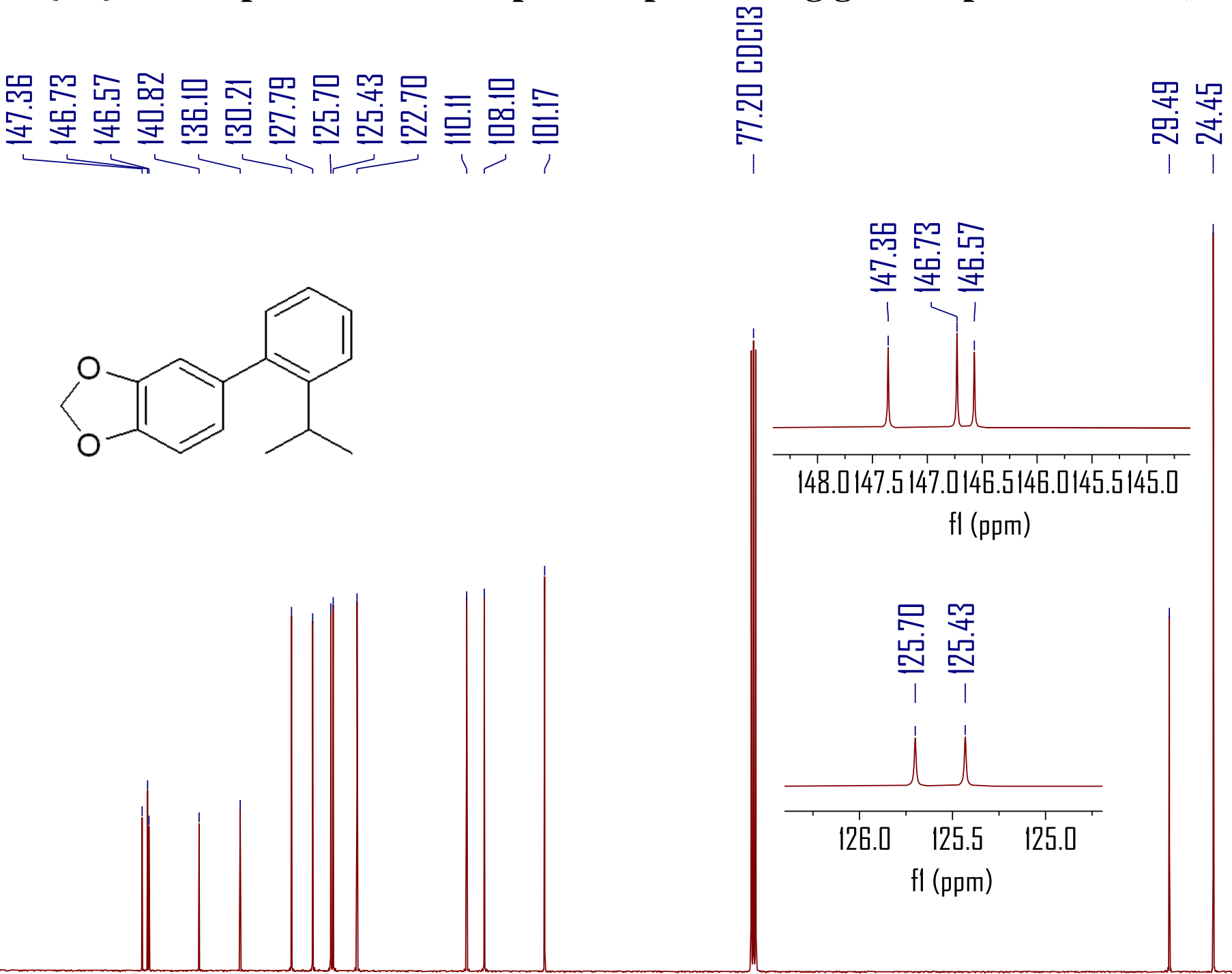

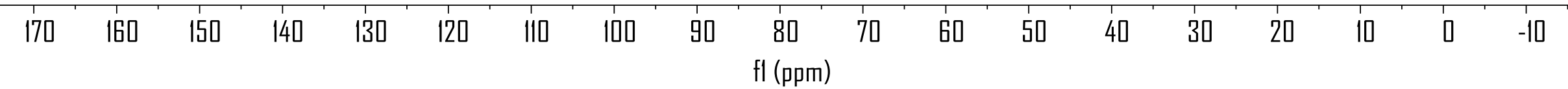




\section{${ }^{1} \mathrm{H}$ NMR spectrum for compound 3p following general procedure $\mathrm{B}\left(400 \mathrm{MHz}, \mathrm{CDCl}_{3}\right)$}

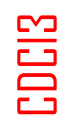

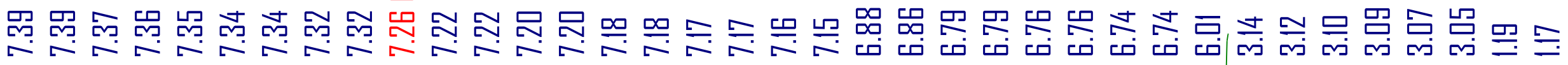
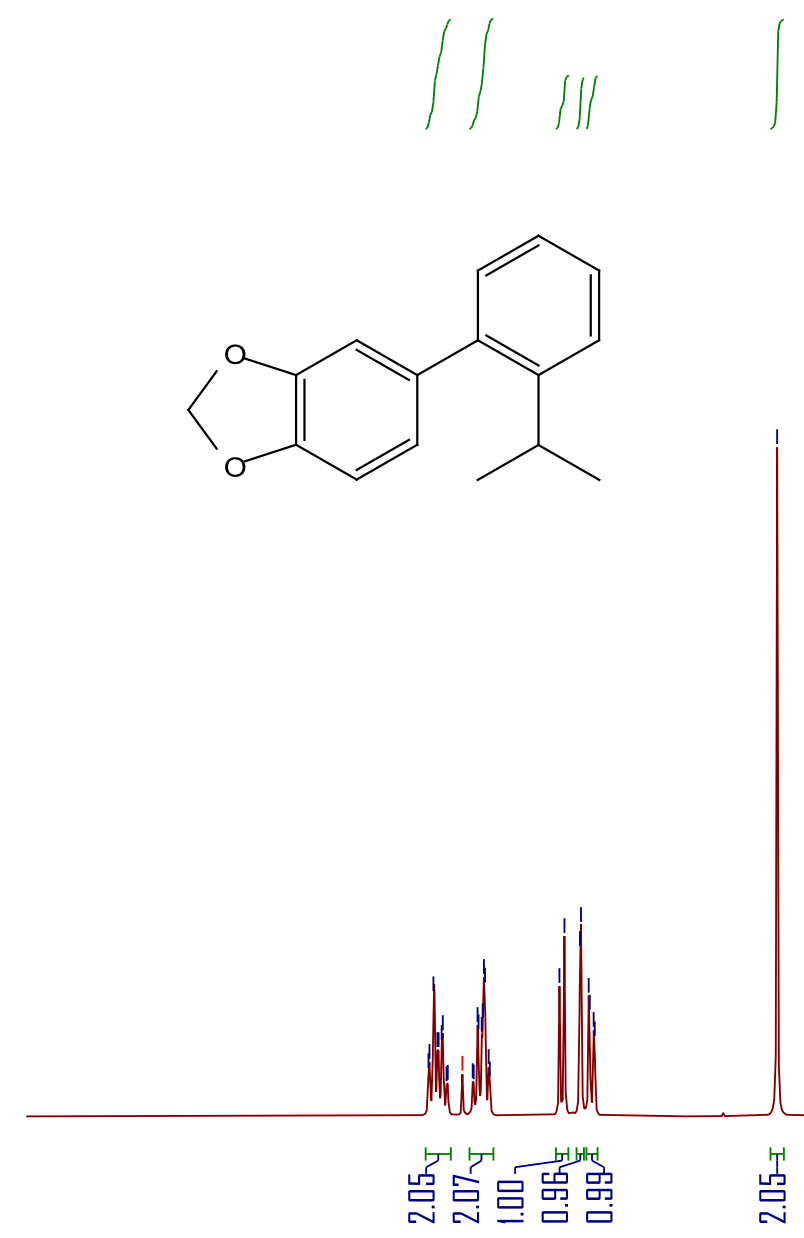

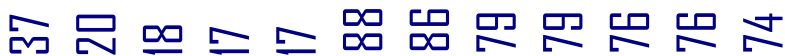
ऽ

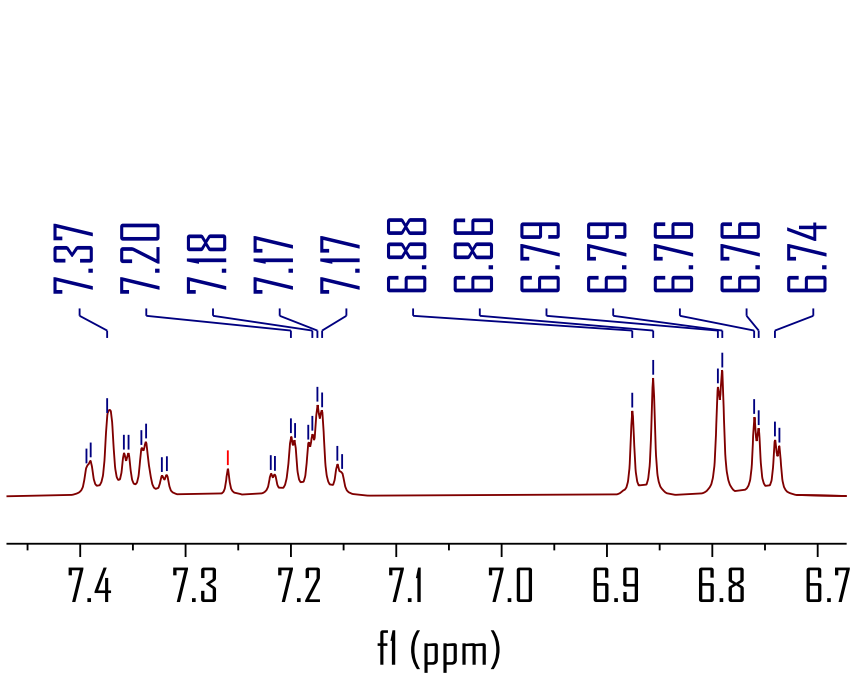

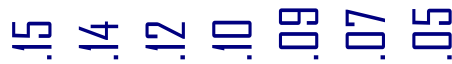

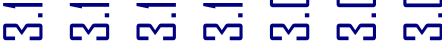

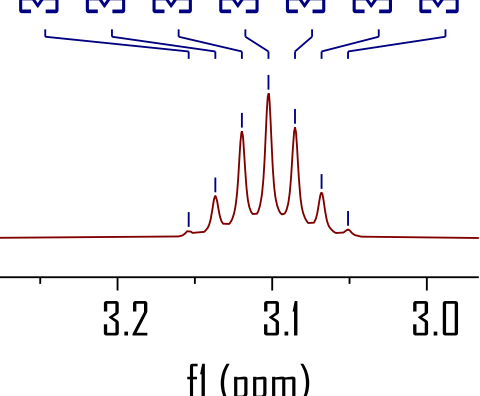

8.5

$8.0 \quad 7.5$

$7.0 \quad 6.5$

6.0 5.5

5.5

$5.0 \quad 4.5$

4.5

fl (ppm)

贺 
${ }^{13} \mathrm{C}\left\{{ }^{1} \mathrm{H}\right\}$ NMR spectrum for compound 3p following general procedure $\mathrm{B}\left(100 \mathrm{MHz}, \mathrm{CDCl}_{3}\right)$

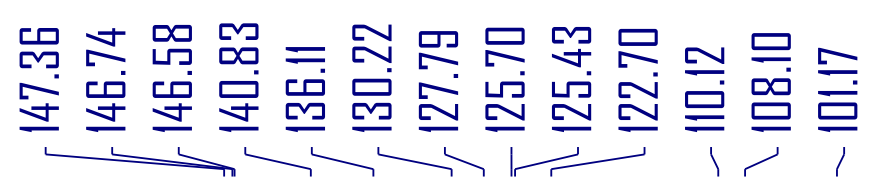

몸
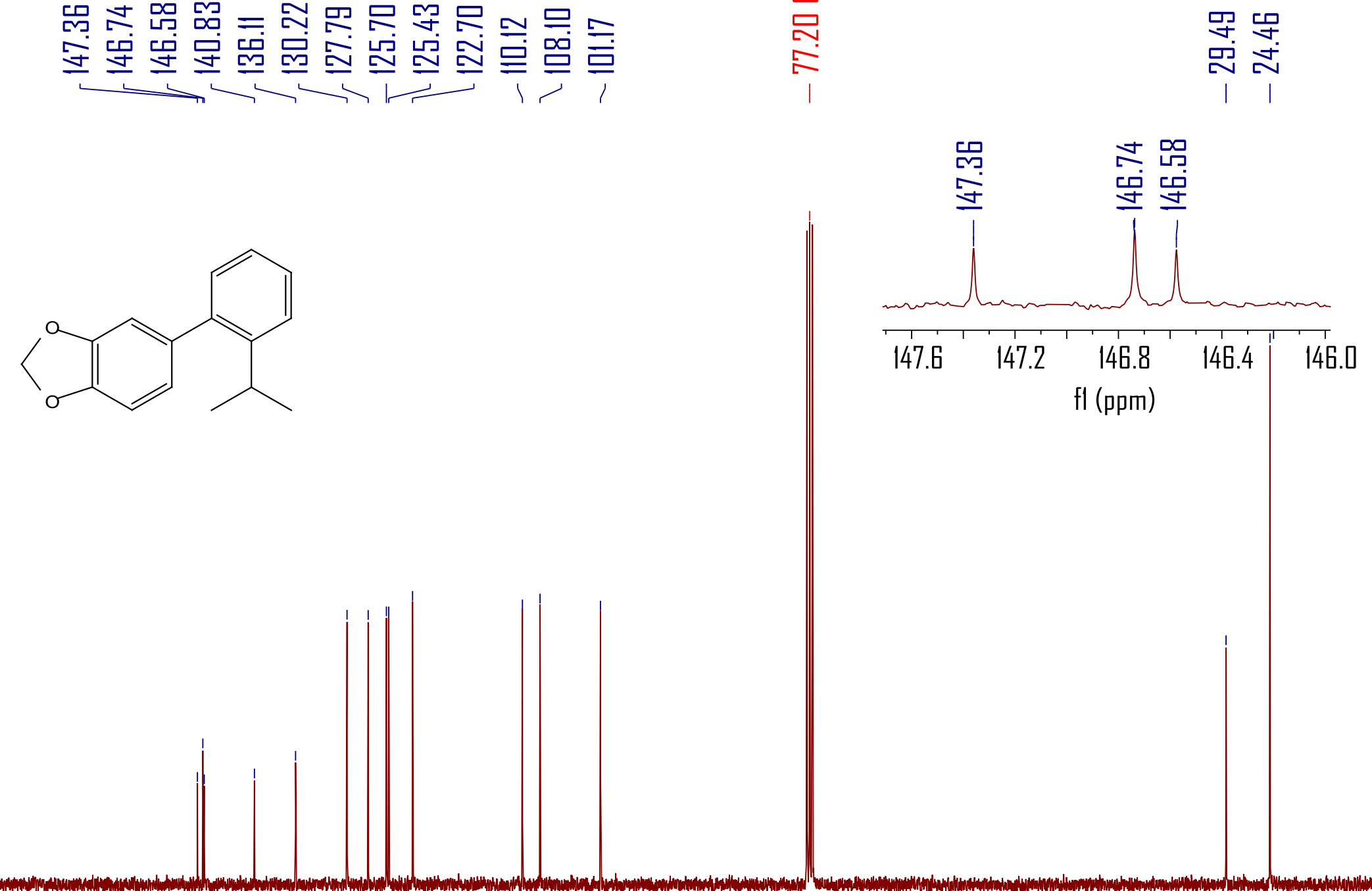

120

110

100

90

$\mathrm{fl}(\mathrm{ppm})$

70

6




\section{${ }^{1} \mathrm{H}$ NMR spectrum for compound 3q (500 $\left.\mathrm{MHz}, \mathrm{CDCl}_{3}\right)$}

\section{営}

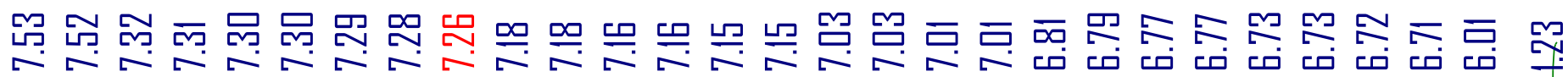

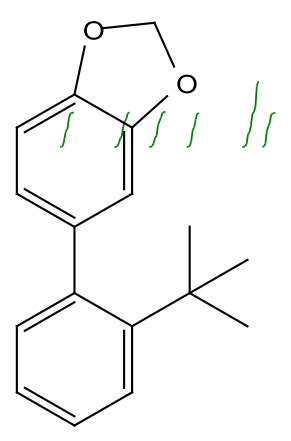

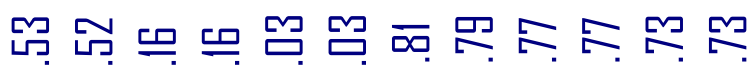

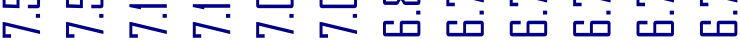
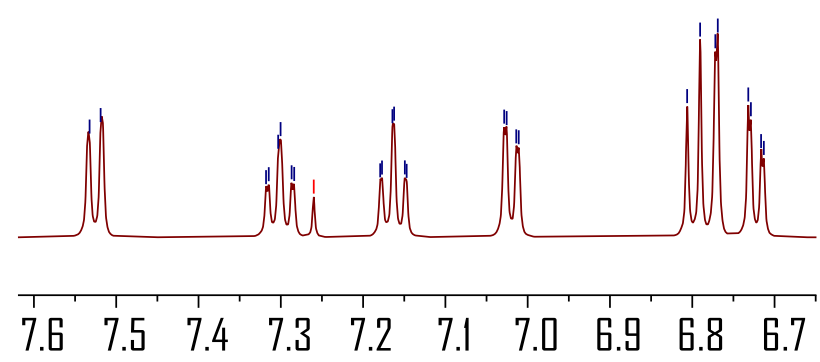

$\mathrm{fl}(\mathrm{ppm})$

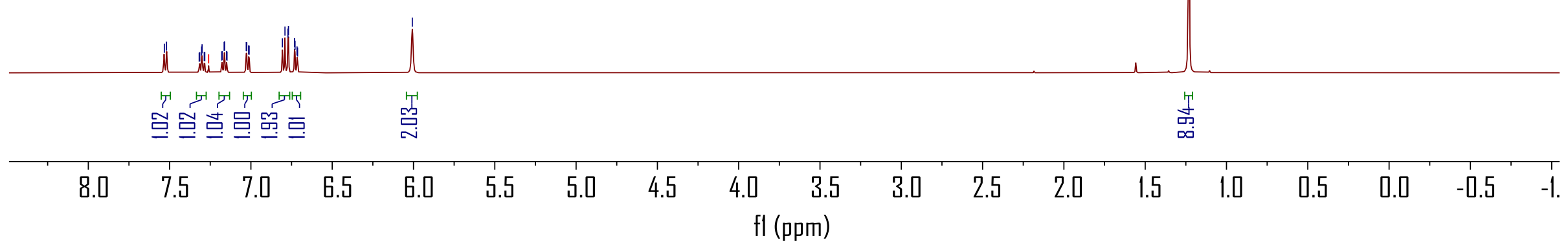




\section{${ }^{13} \mathrm{C}\left\{{ }^{1} \mathrm{H}\right\}$ NMR spectrum for compound 3q (125 $\left.\mathrm{MHz}, \mathrm{CDCl}_{3}\right)$}

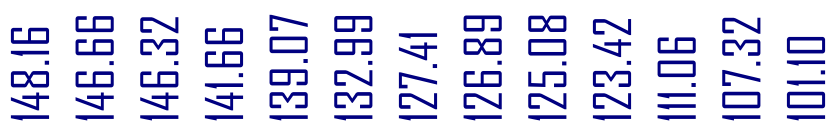

몰

$\approx \stackrel{5}{\infty}$

菂
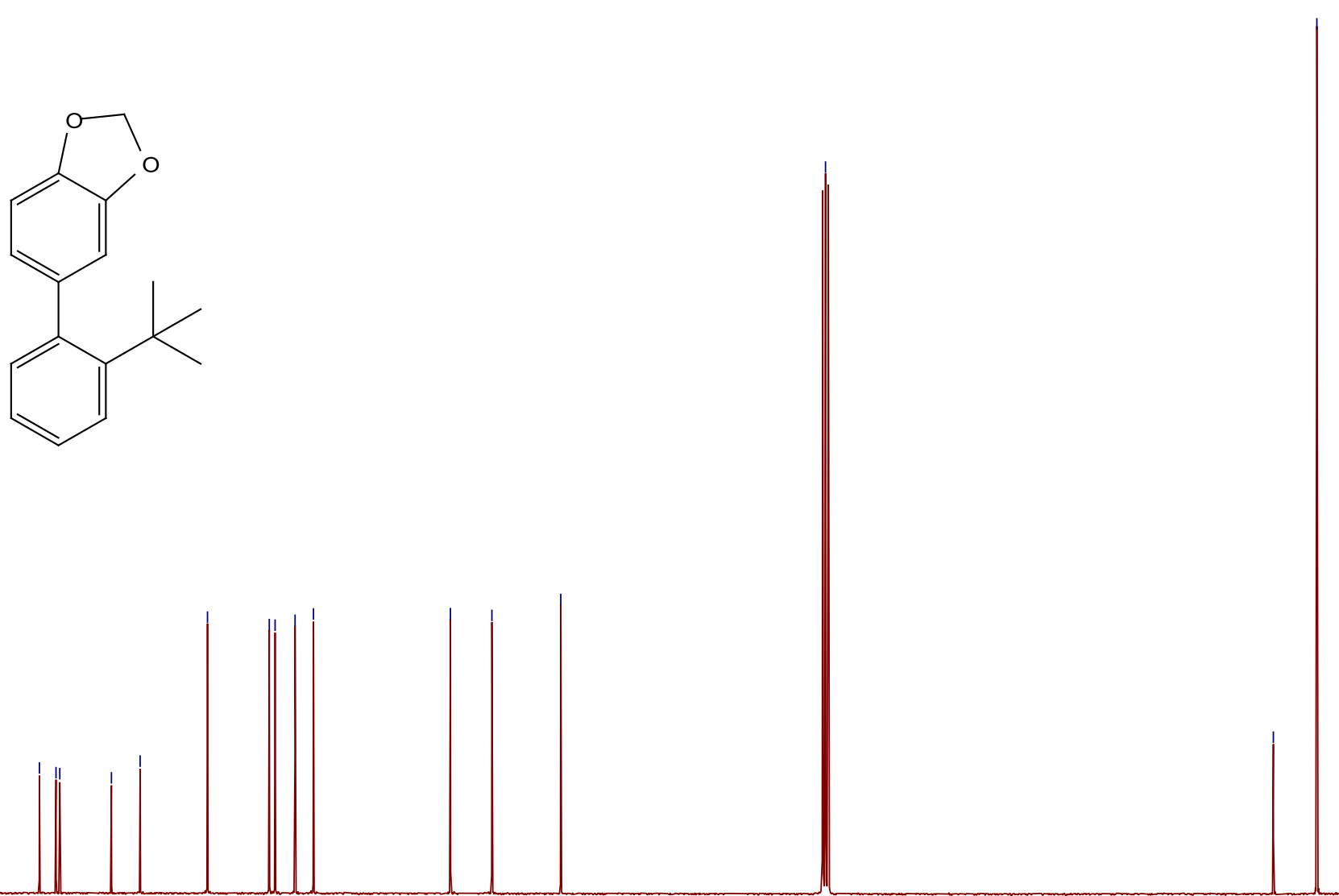

170

130

120

110

100

90 


\section{${ }^{1} \mathrm{H}$ NMR spectrum for compound $3 \mathrm{r}\left(500 \mathrm{MHz}, \mathrm{CDCl}_{3}\right)$}

\section{믐}

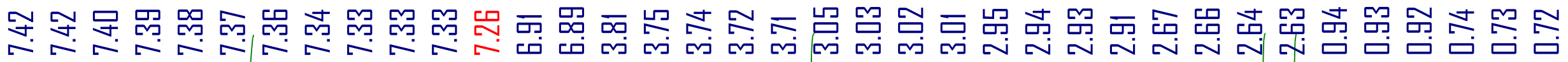
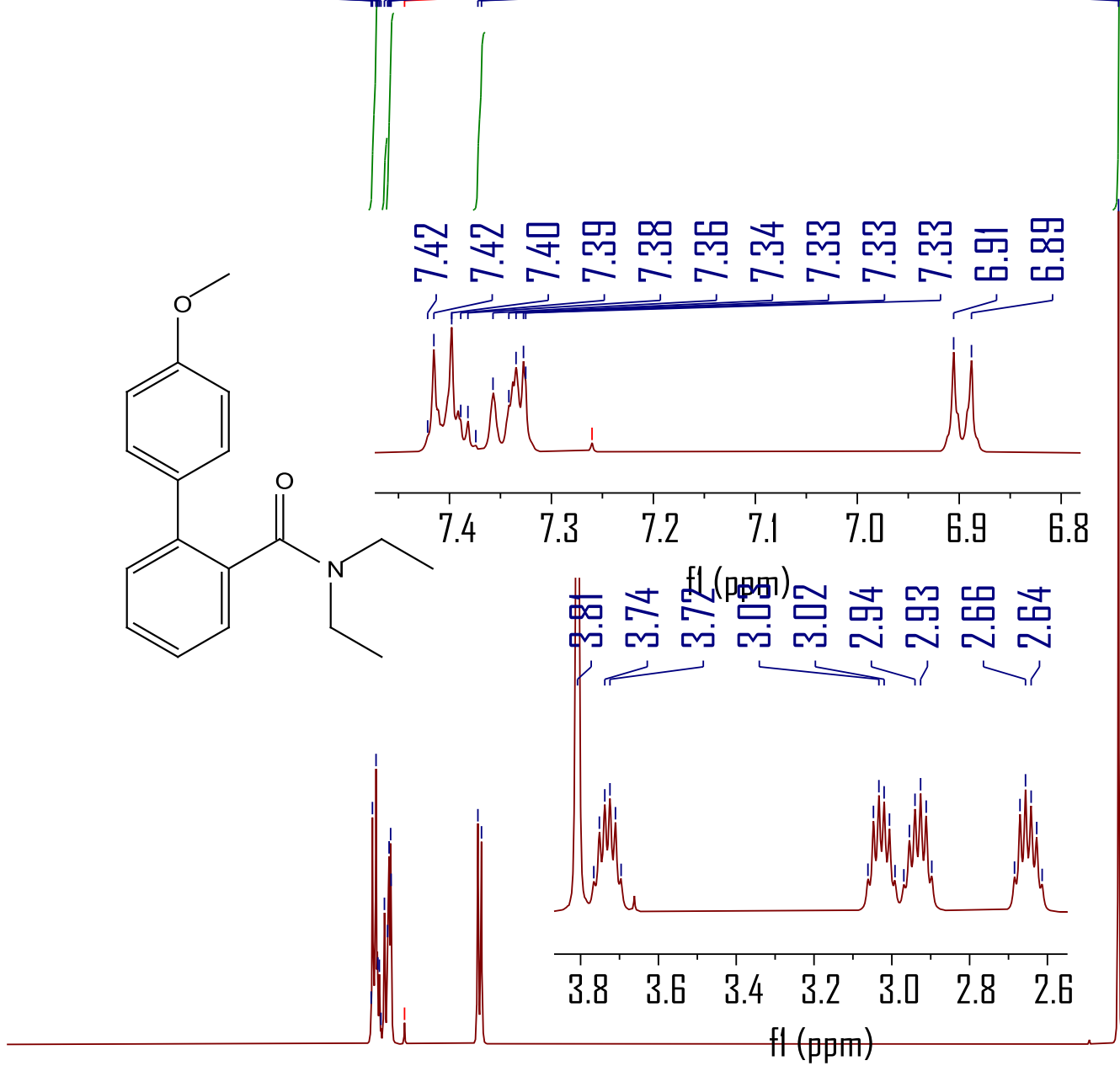

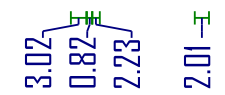

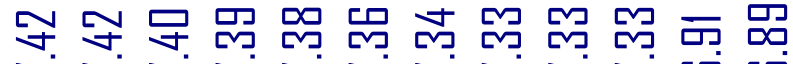

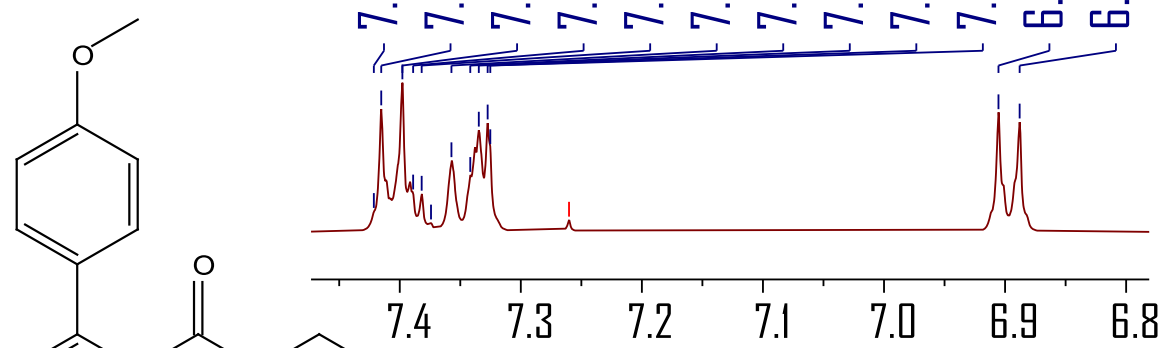

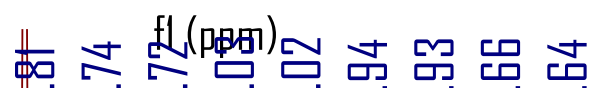

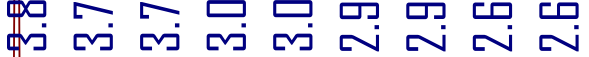
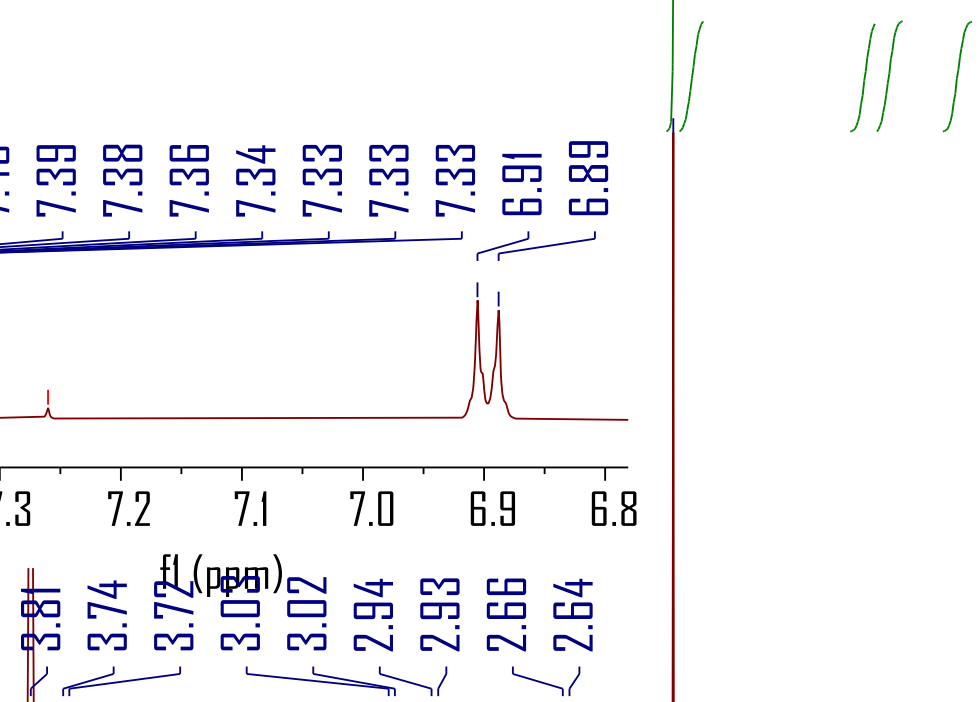

\section{8}

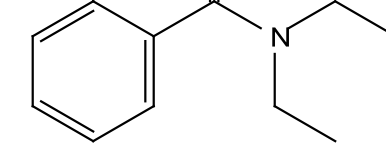

fl (ppm)

第鮆

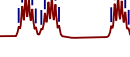

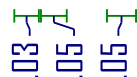

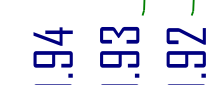

高骂 品

$\underbrace{\square}_{1}$

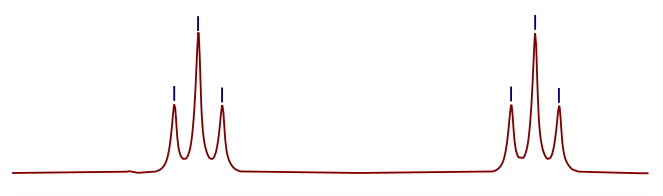

1.000 .950 .900 .850 .800 .750 .70 $\mathrm{fl}(\mathrm{ppm})$

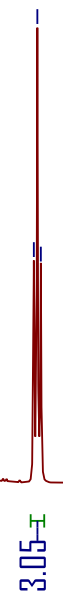

\begin{tabular}{ccccccccccccccccccccc}
\hline 9.0 & 8.5 & 8.0 & 7.5 & 7.0 & 6.5 & 6.0 & 5.5 & 5.0 & 4.5 & $\begin{array}{c}4.0 \\
\mathrm{fl}(\mathrm{ppm}) \\
\mathrm{s} 110\end{array}$ & 3.5 & 3.0 & 2.5 & 2.0 & 1.5 & 1.0 & 0.5 & 0.0 & -0.5 & -1.
\end{tabular}




\section{${ }^{13} \mathrm{C}\left\{{ }^{1} \mathrm{H}\right\}$ NMR spectrum for compound 3r (125 $\left.\mathrm{MHz}, \mathrm{CDCl}_{3}\right)$}

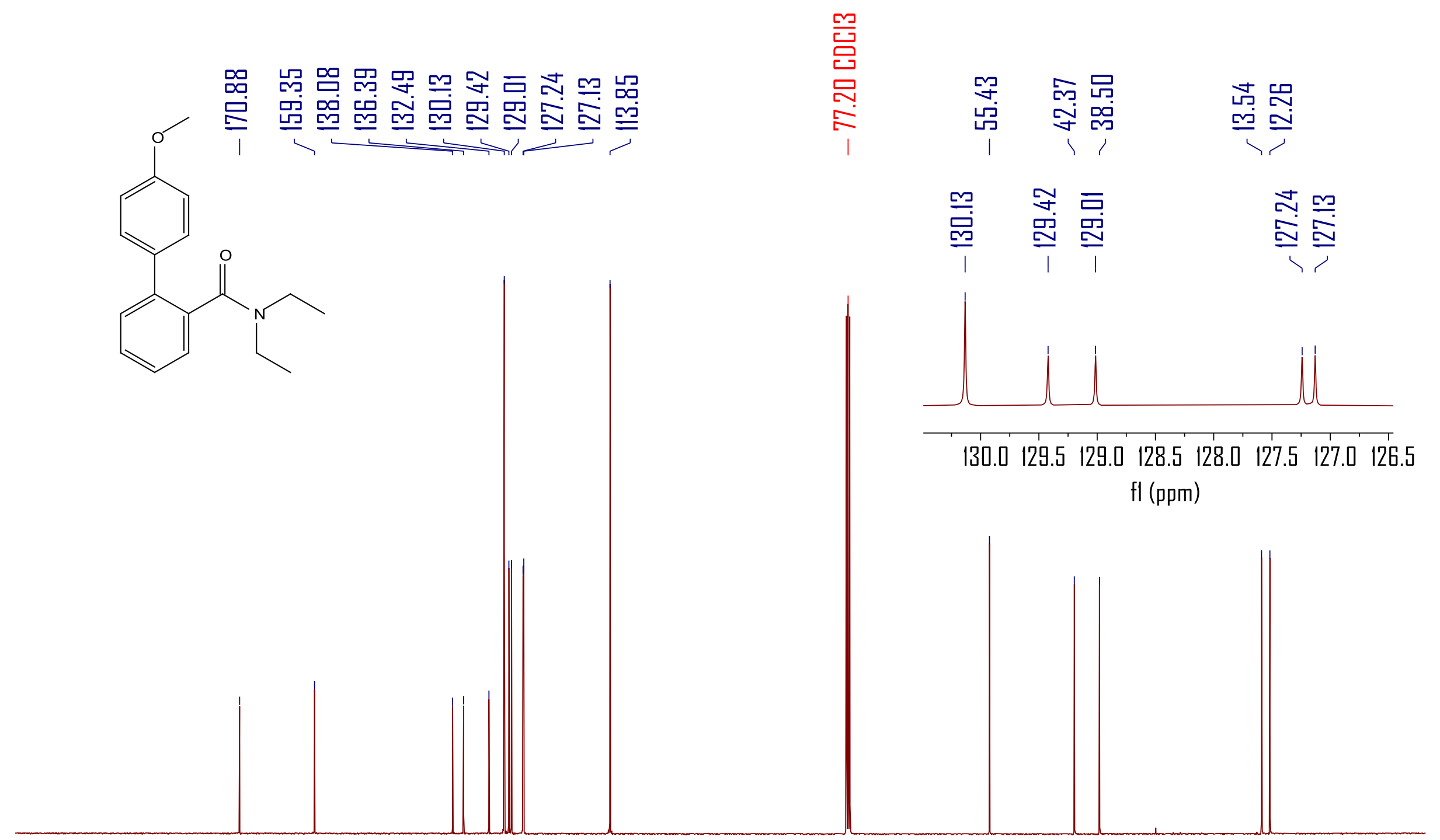

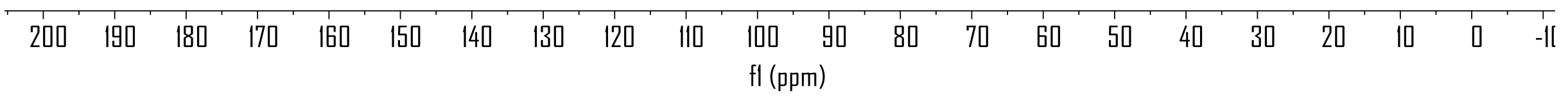




\section{${ }^{1} \mathrm{H}$ NMR spectrum for compound 3s (500 $\mathrm{MHz}, \mathrm{CDCl}_{3}$, TMS)}

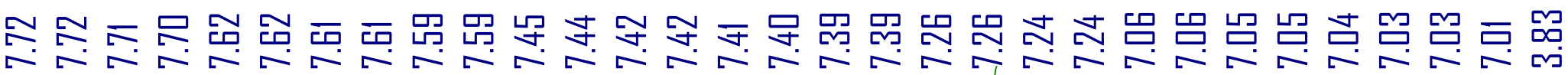

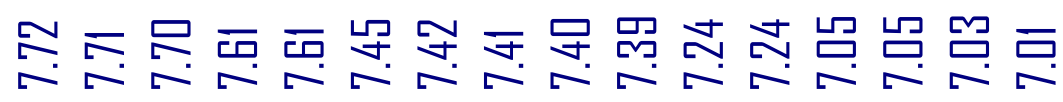
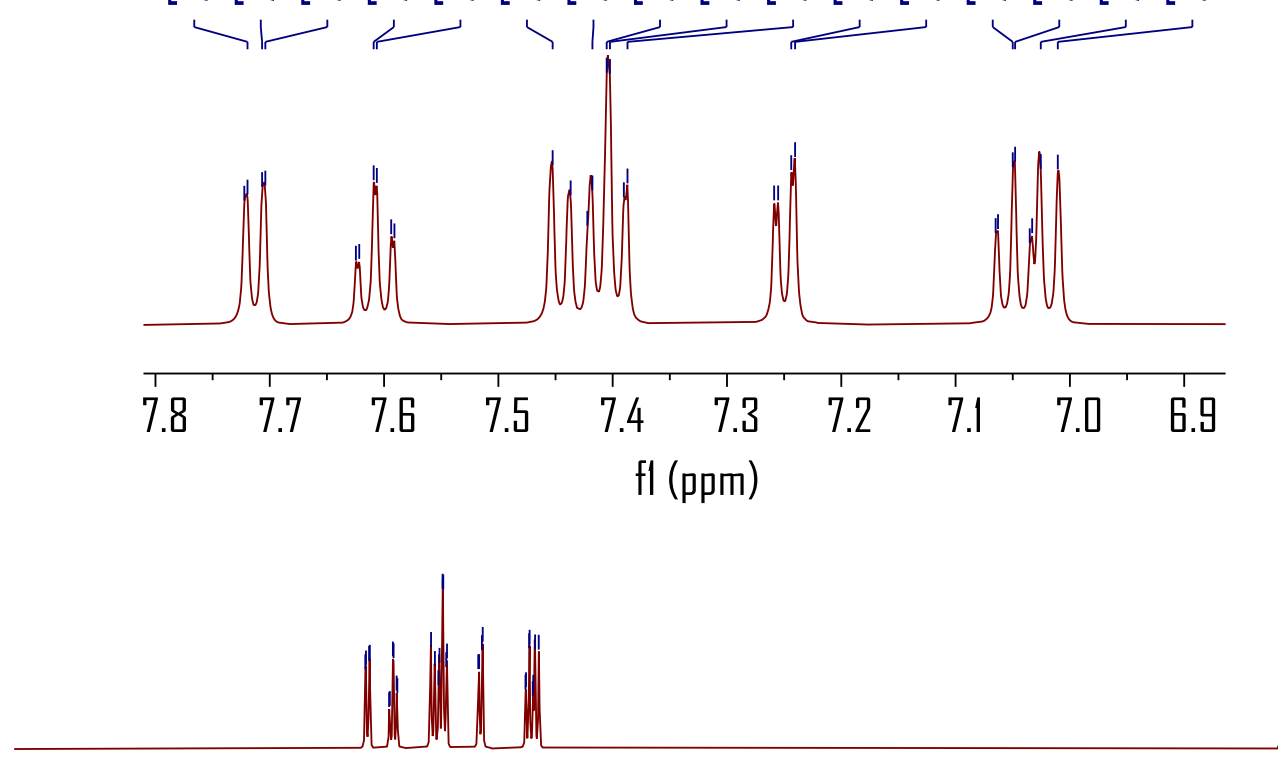

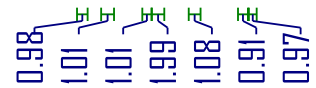

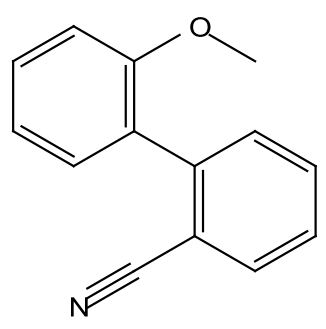

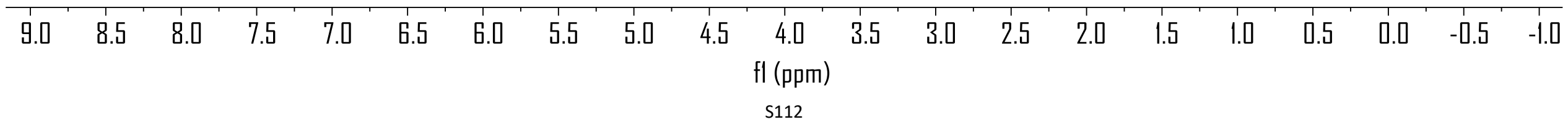




\section{${ }^{13} \mathrm{C}\left\{{ }^{1} \mathrm{H}\right\}$ NMR spectrum for compound 3s (100 $\left.\mathrm{MHz}, \mathrm{CDCl}_{3}\right)$}

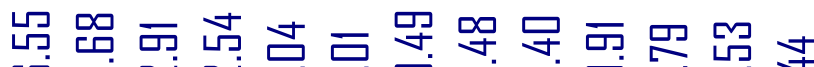

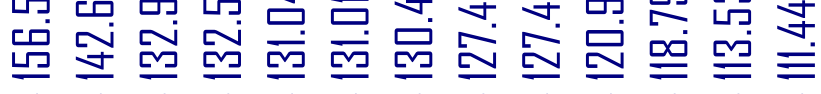

無
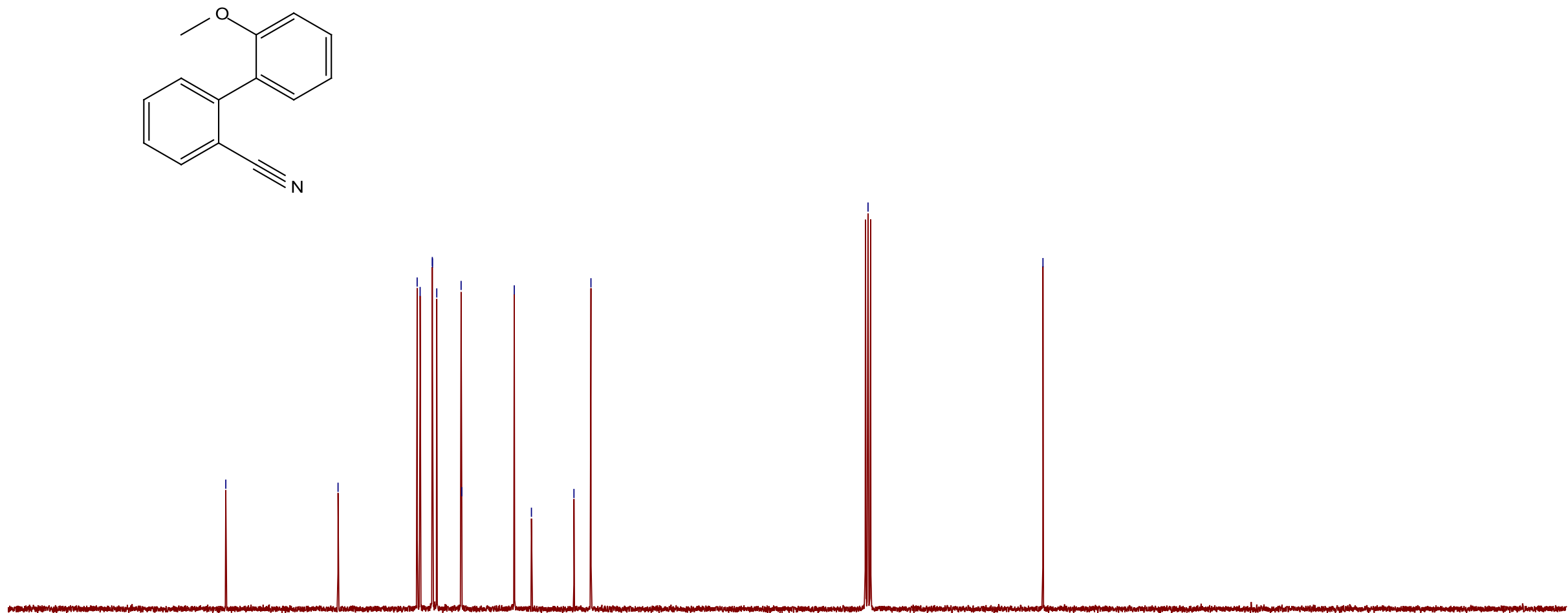

מִ 


\section{${ }^{1} \mathrm{H}$ NMR spectrum for compound $3 \mathrm{t}\left(400 \mathrm{MHz}, \mathrm{CDCl}_{3}, \mathrm{TMS}\right)$}

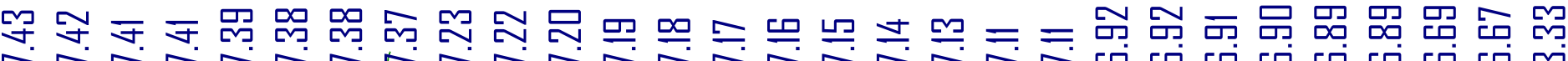

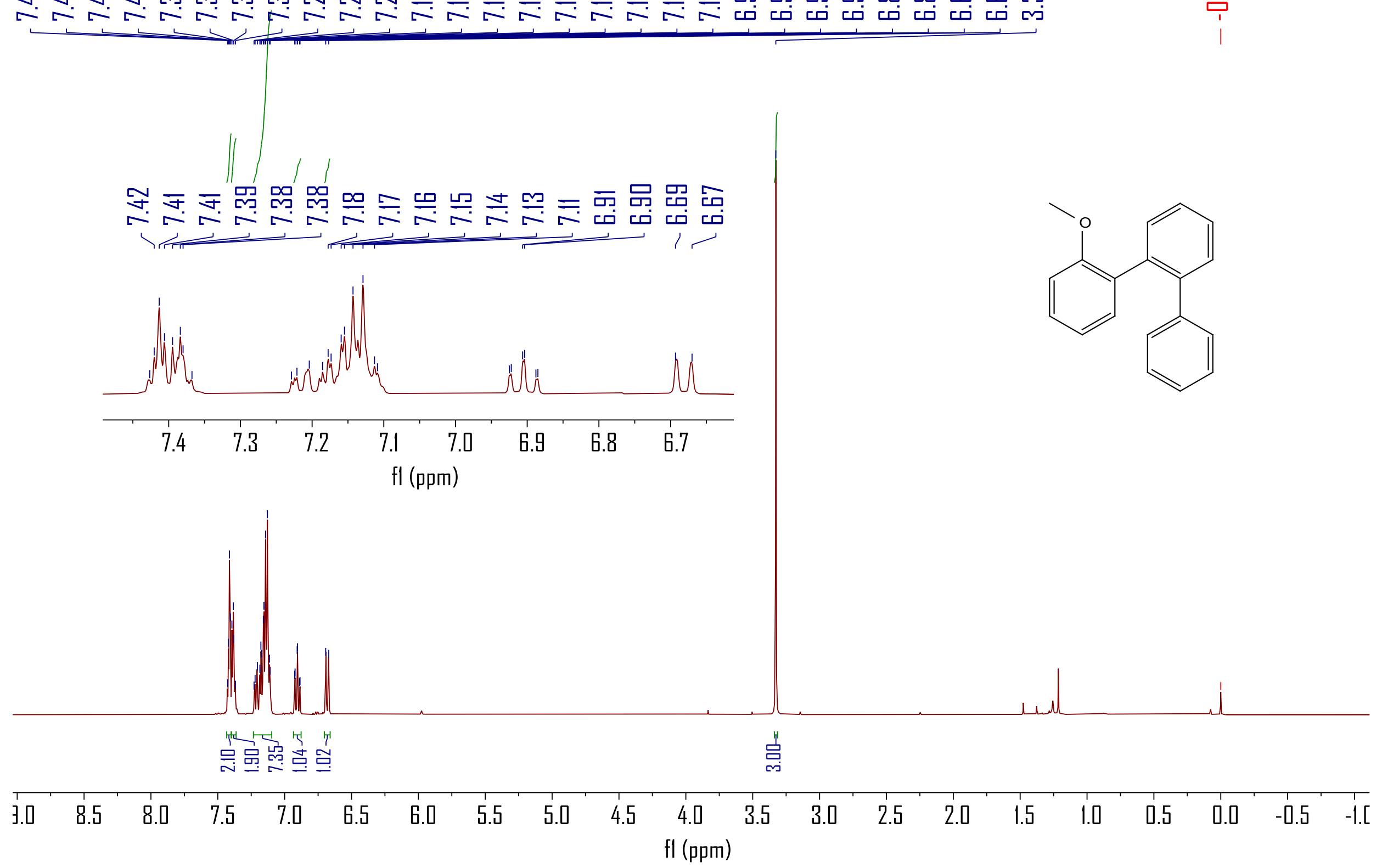




\section{${ }^{13} \mathrm{C}\left\{{ }^{1} \mathrm{H}\right\}$ NMR spectrum for compound 3t (125 $\left.\mathrm{MHz}, \mathrm{CDCl}_{3}\right)$}

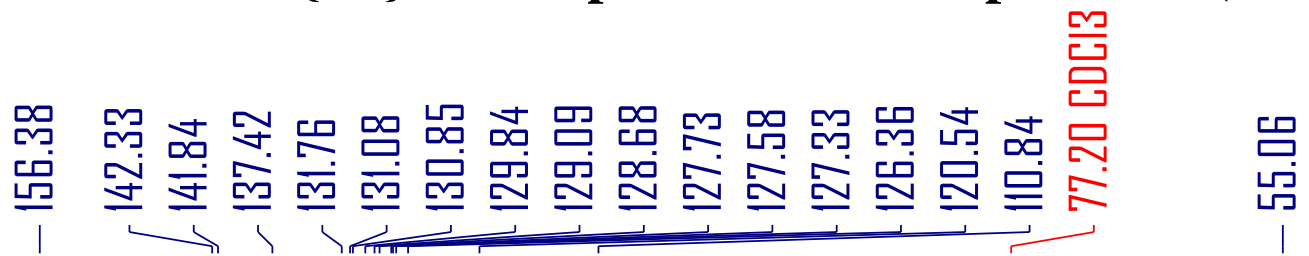

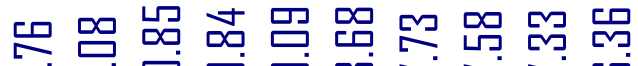

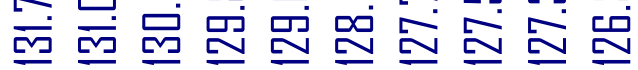
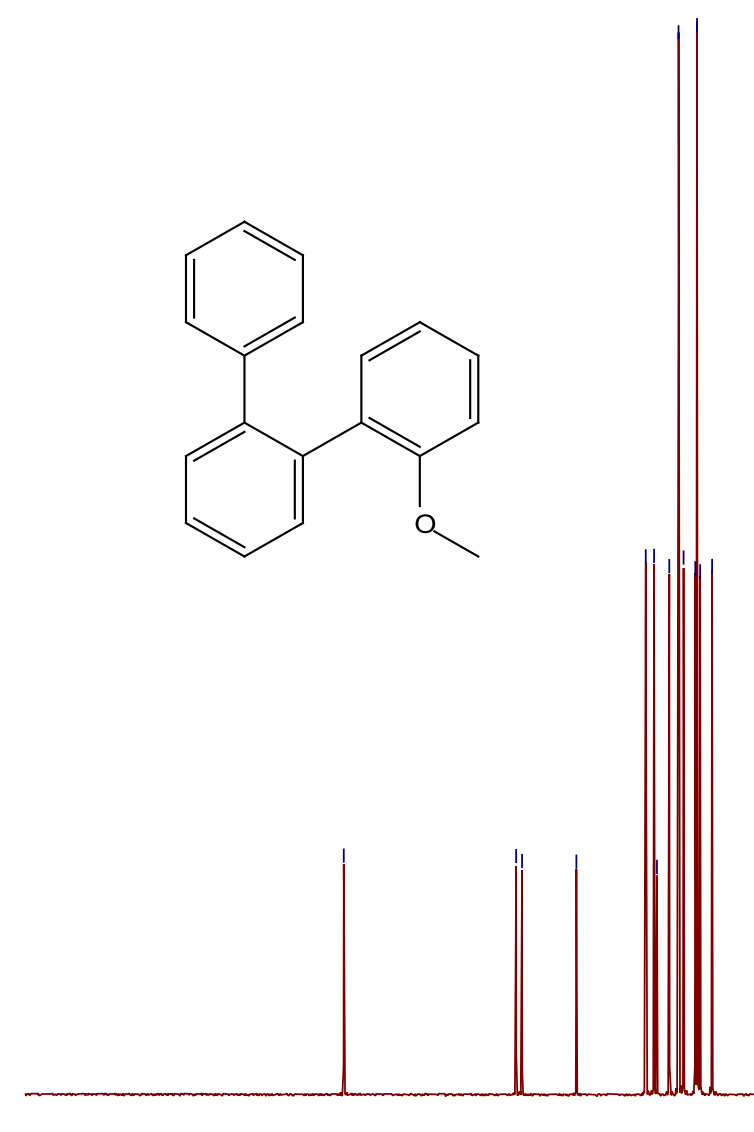

$15 \div-\frac{1}{1} \div 1$
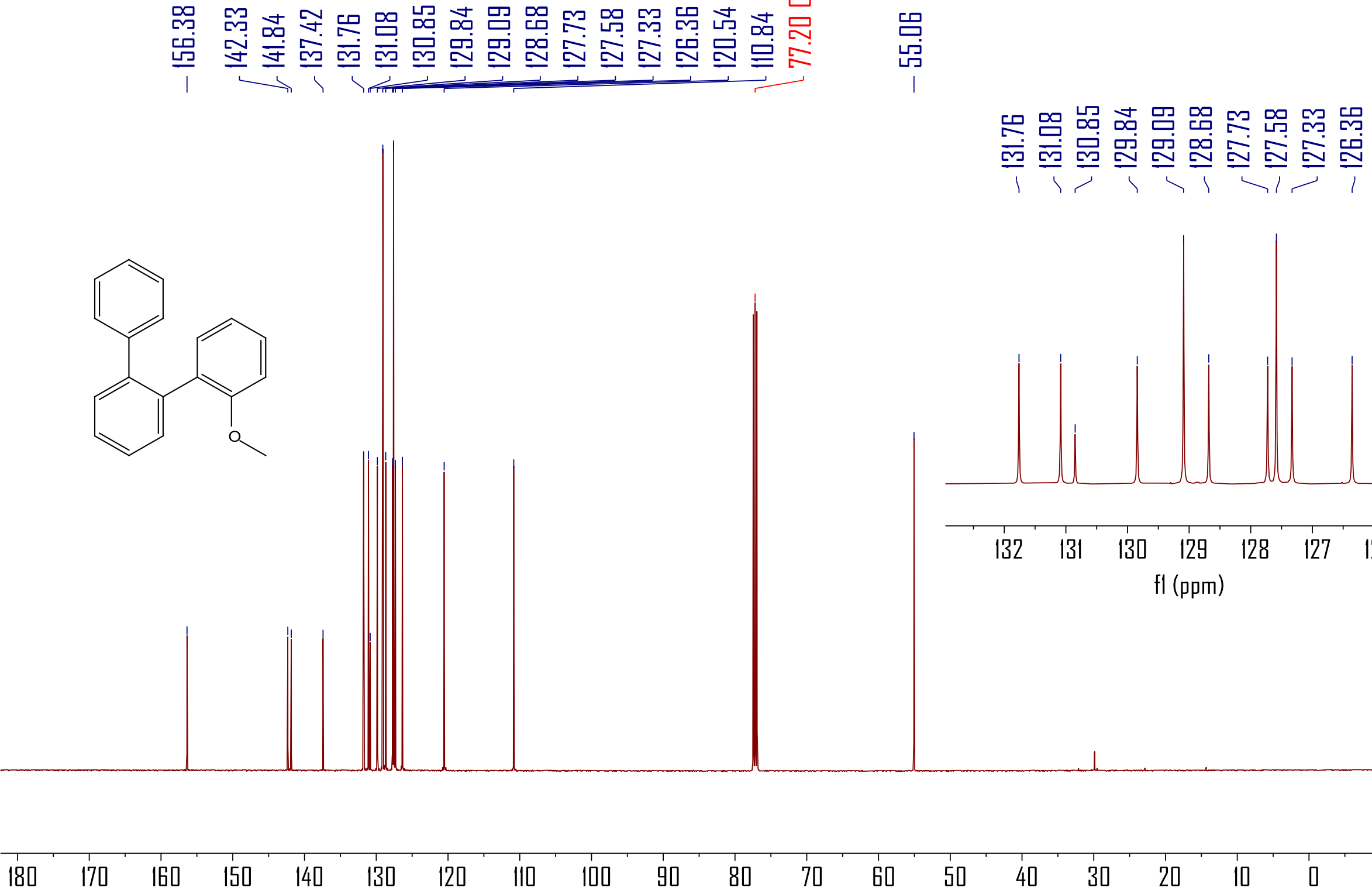

130

120

$+1$
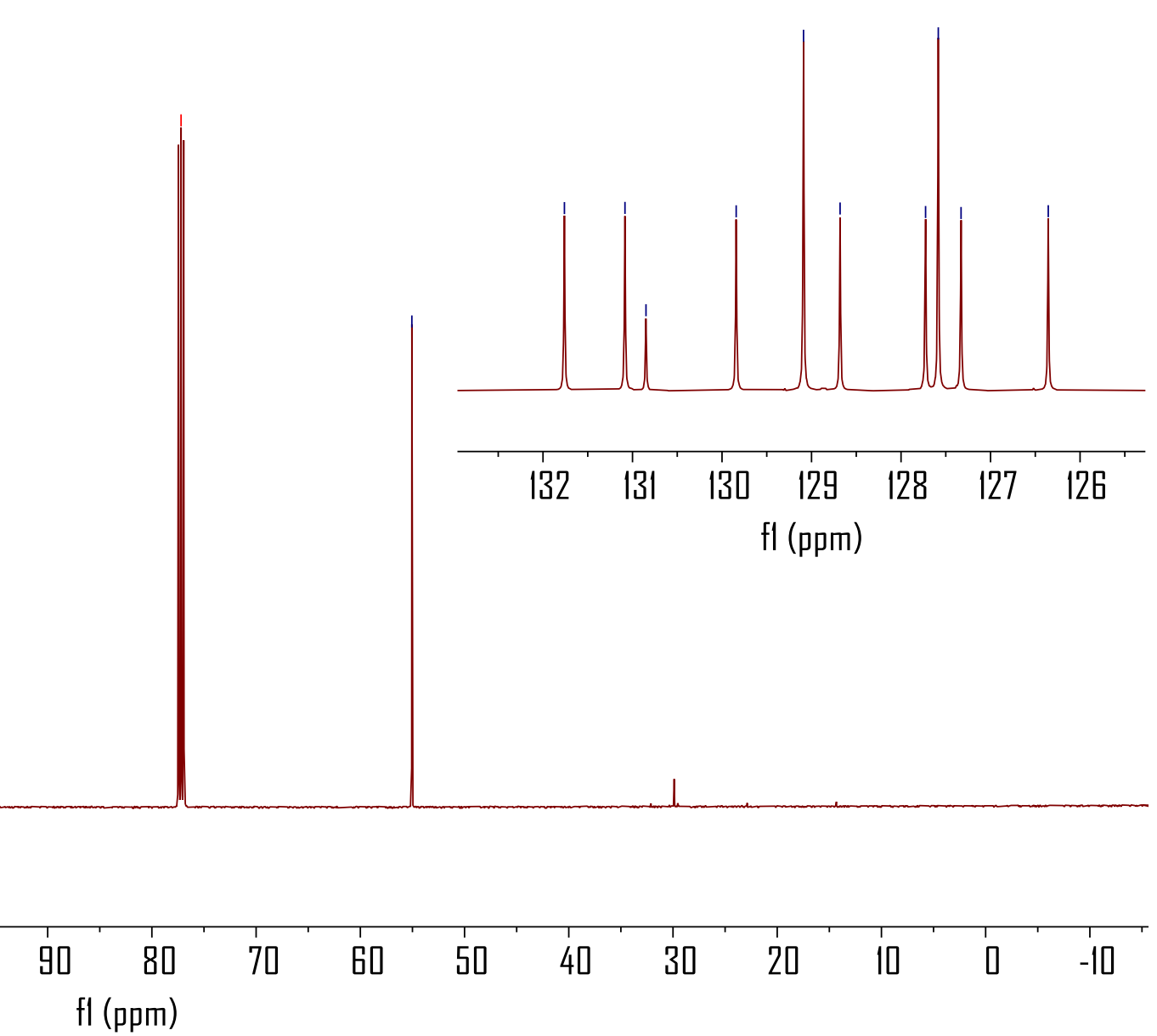


\section{${ }^{1} \mathrm{H}$ NMR spectrum for compound $3 \mathrm{u}\left(500 \mathrm{MHz}^{\mathrm{CDCl}} \mathrm{CD}_{3}\right.$}

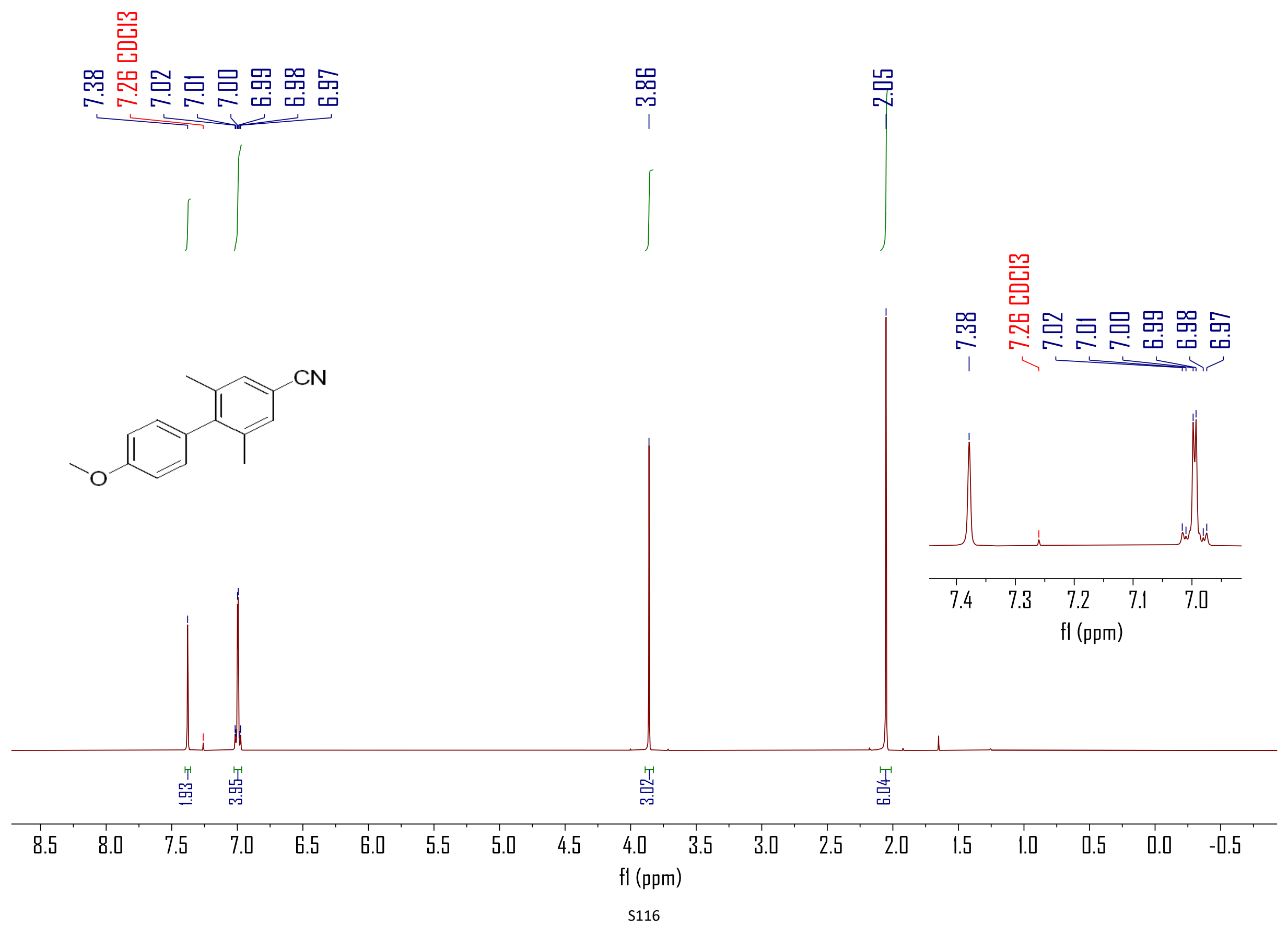


${ }^{13} \mathrm{C}\left\{{ }^{1} \mathrm{H}\right\}$ NMR spectrum for compound $3 \mathrm{u}\left(125 \mathrm{MHz}, \mathrm{CDCl}_{3}\right)$

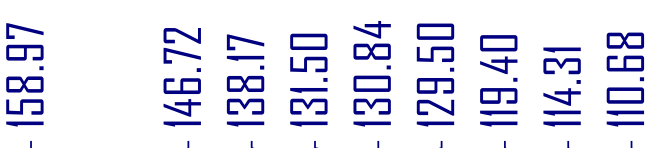

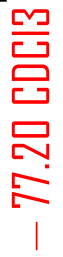

品

虫

茗
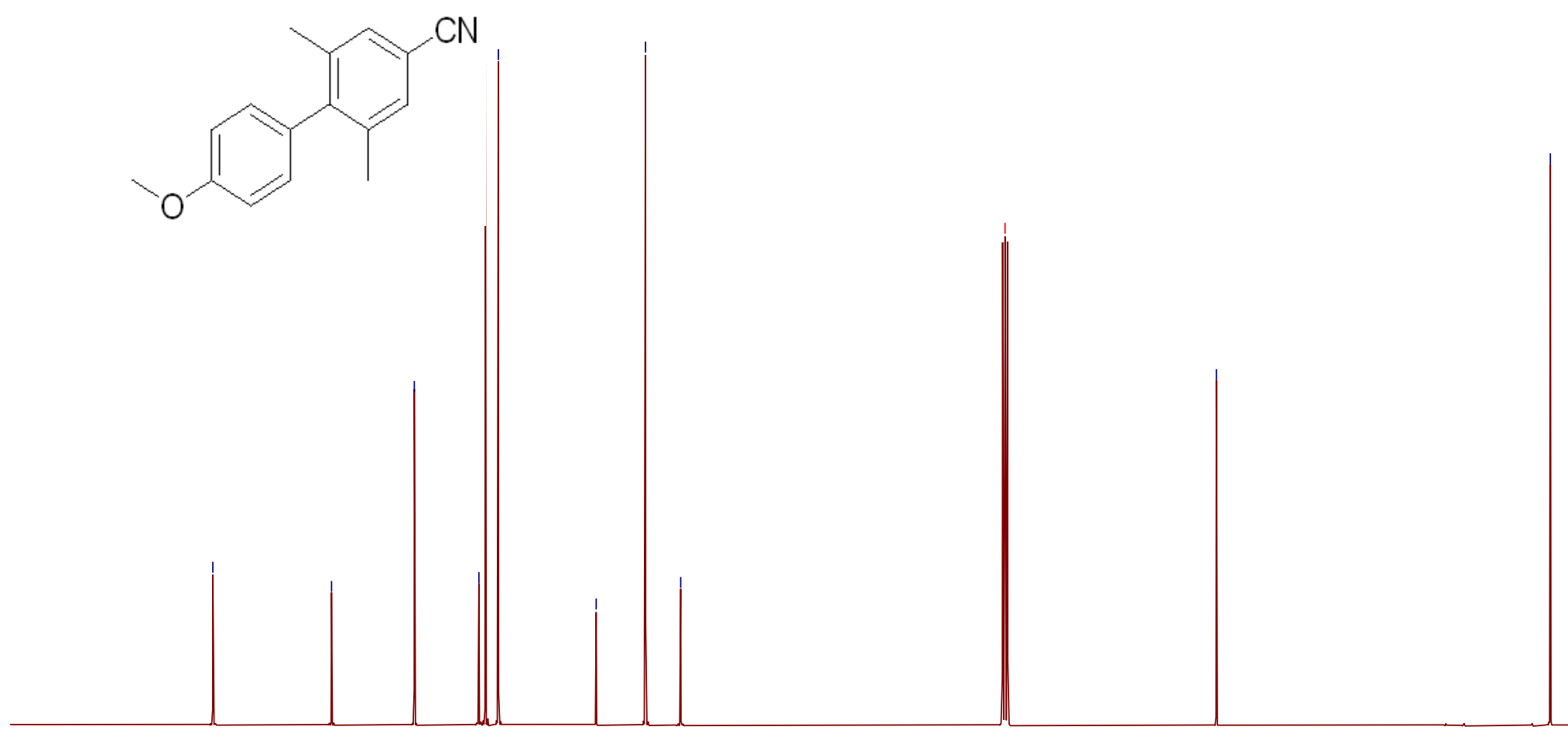


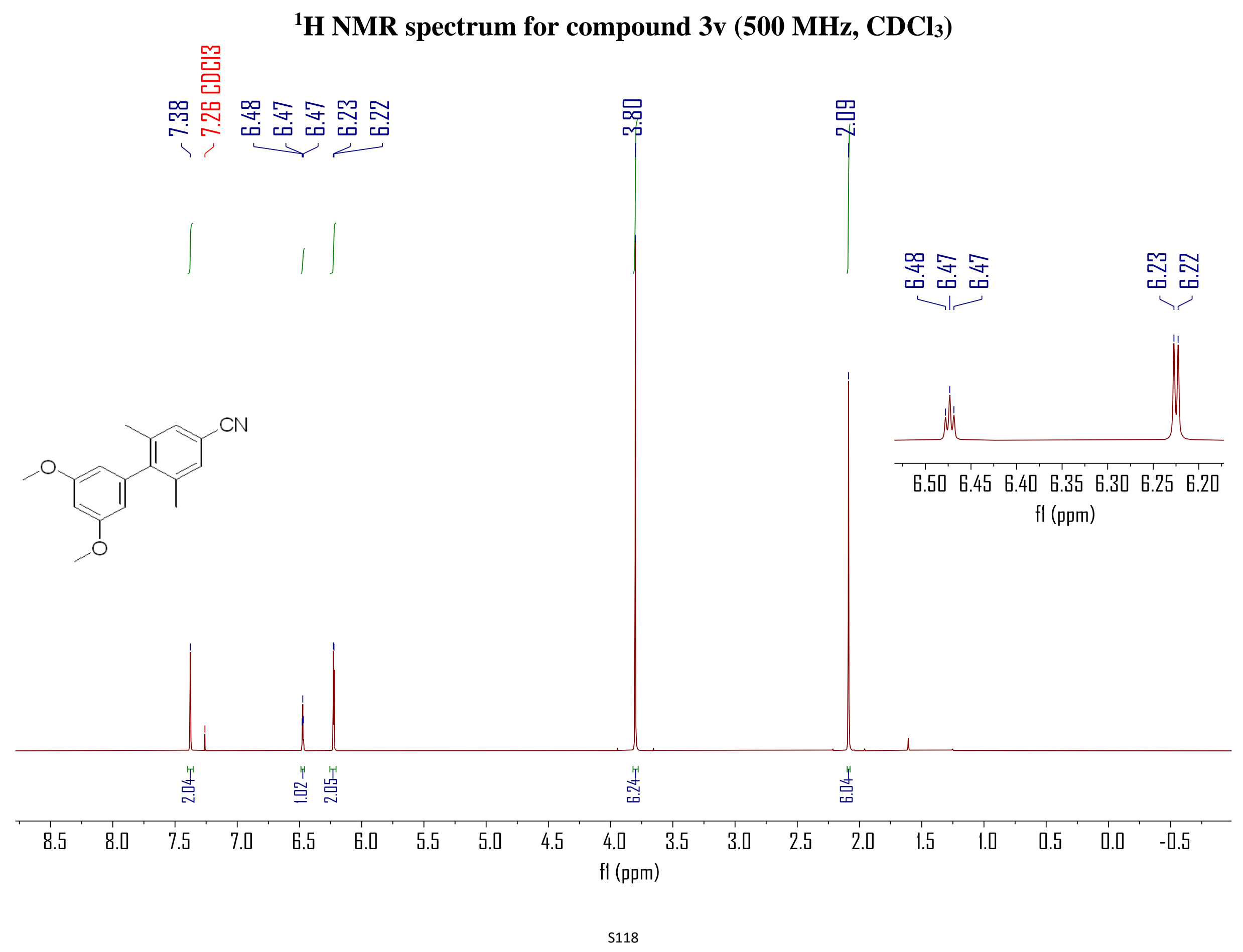




\section{${ }^{13} \mathrm{C}\left\{{ }^{1} \mathrm{H}\right\} \mathrm{NMR}$ spectrum for compound 3v (125 $\left.\mathrm{MHz}, \mathrm{CDCl}_{3}\right)$}

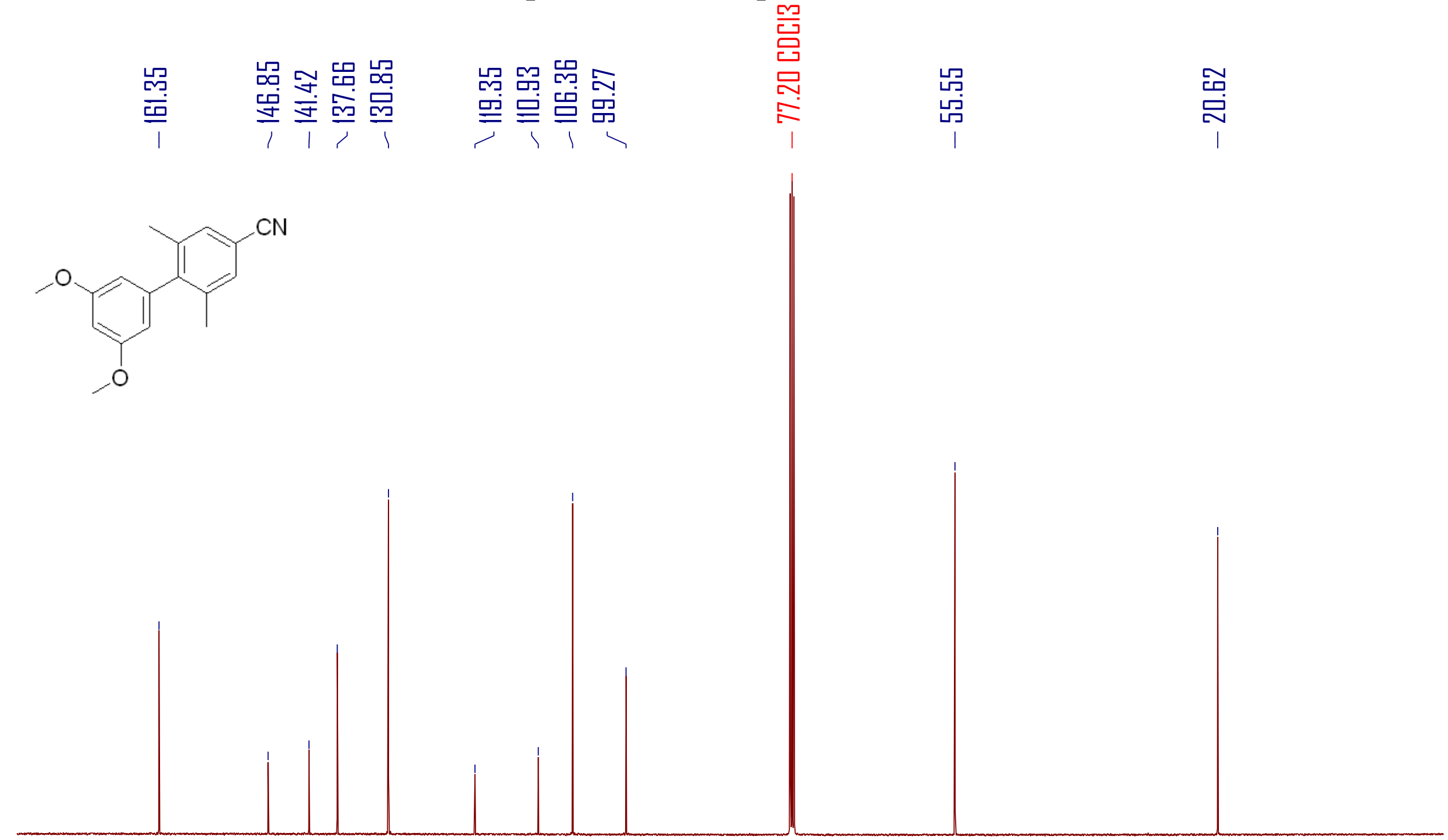

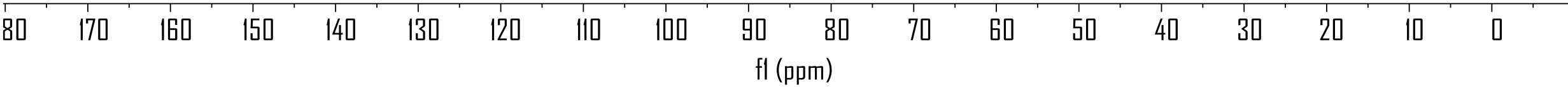




\section{${ }^{1} \mathrm{H}$ NMR spectrum for compound 3w following general procedure $\mathrm{A}(400 \mathrm{MHz}, \mathrm{CDCl}, \mathrm{TMS})$}

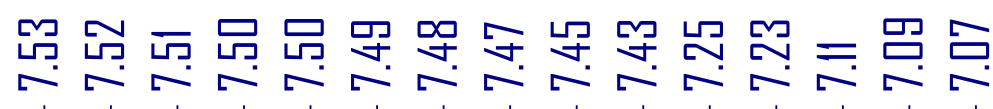
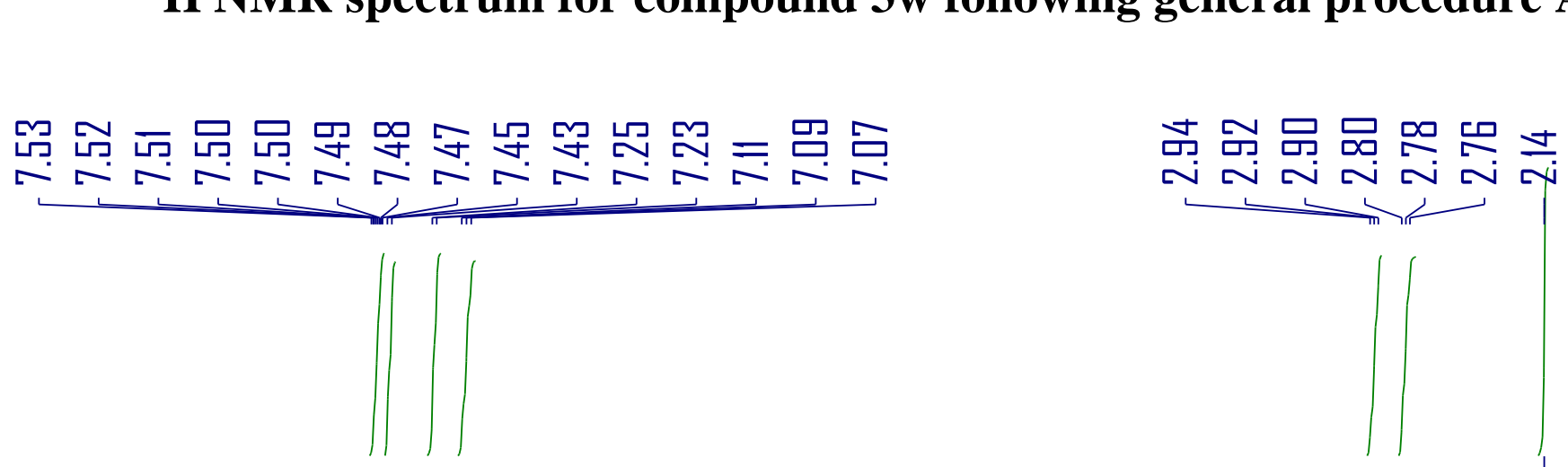

点

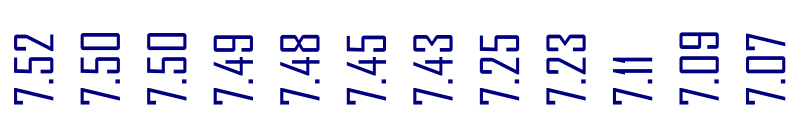

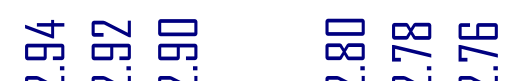

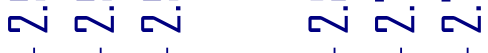

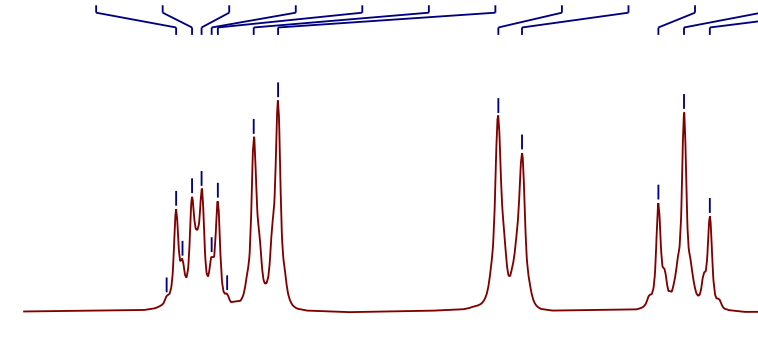

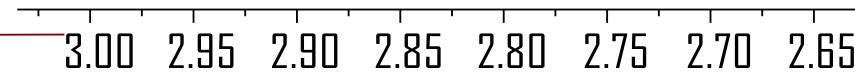

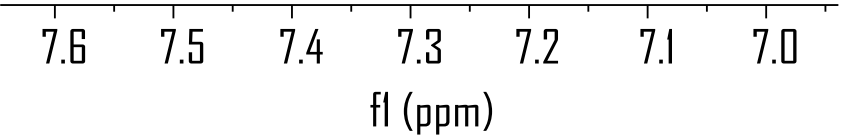

$\mathrm{fl}$ (ppm)

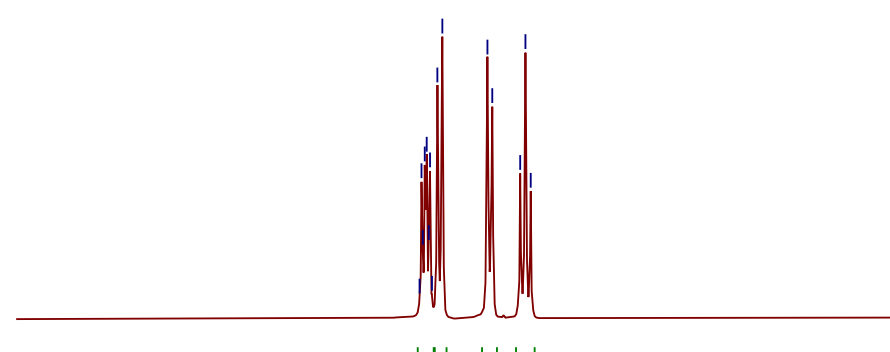

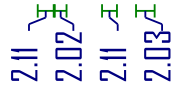

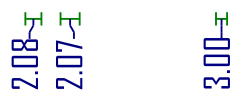
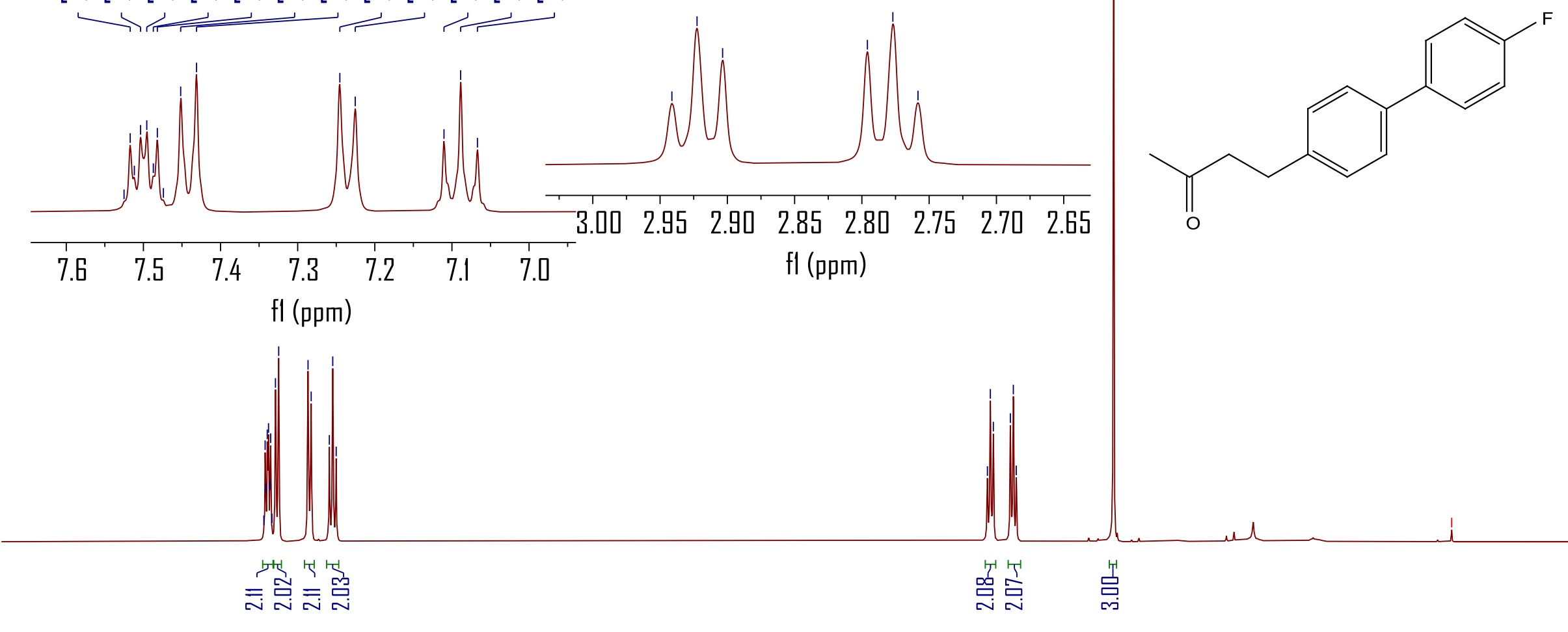

$9.0 \quad 8.5 \quad 8.5$

$\begin{array}{lll}1.5 & 7.0 & 6.5\end{array}$

6.0

$5.5 \quad 5.0$

4.5




\section{${ }^{13} \mathrm{C}\left\{{ }^{1} \mathrm{H}\right\}$ NMR spectrum for compound 3w following general procedure $\mathrm{A}\left(100 \mathrm{MHz}, \mathrm{CDCl}_{3}\right)$}
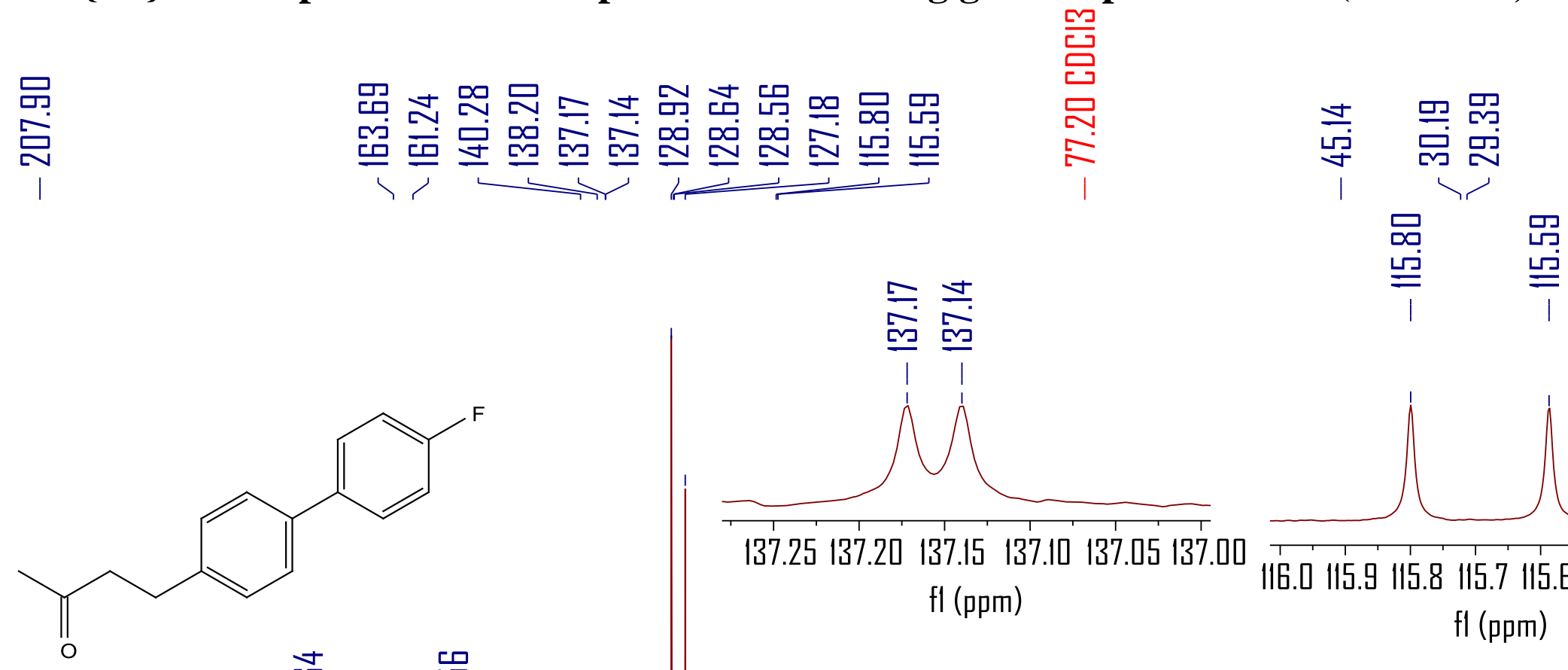

$137.25137 .20137 .15 \quad 137.10137 .05137 .00$ $\mathrm{fl}$ (ppm)

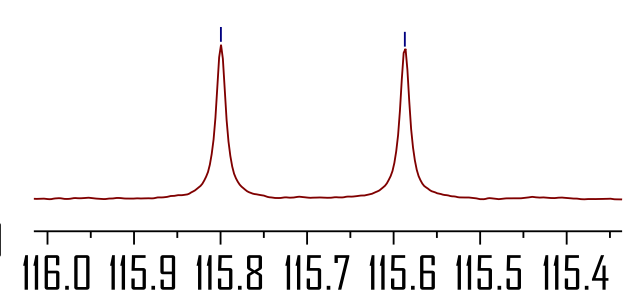

$\mathrm{fl}(\mathrm{ppm})$

$128.70128 .65 \quad 128.60128 .55128 .50$

$\mathrm{fl}(\mathrm{ppm})$

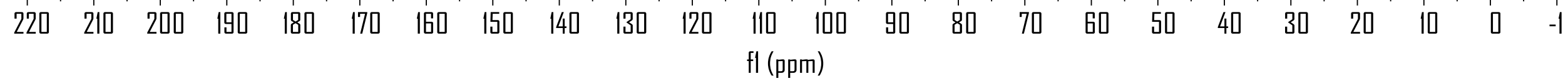


${ }^{19} \mathrm{~F}\left\{{ }^{1} \mathrm{H}\right\}$ NMR spectrum for compound 3w following general procedure $\mathrm{A}\left(376 \mathrm{MHz}, \mathrm{CDCl}_{3}\right)$

号

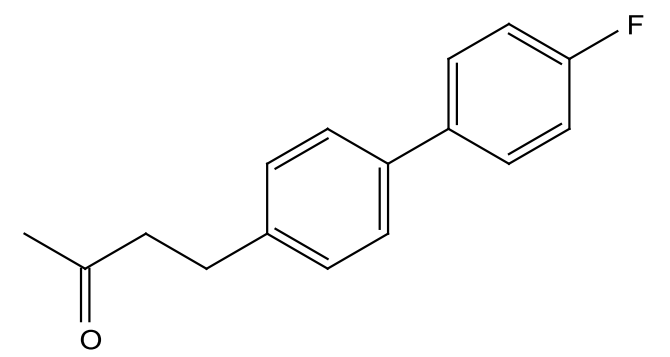

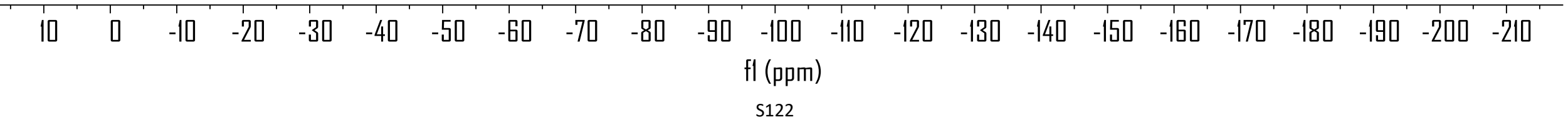




\section{${ }^{1} \mathrm{H}$ NMR spectrum for compound 3w following general procedure $\mathrm{B}\left(400 \mathrm{MHz}, \mathrm{CDCl}_{3}, \mathrm{TMS}\right)$}
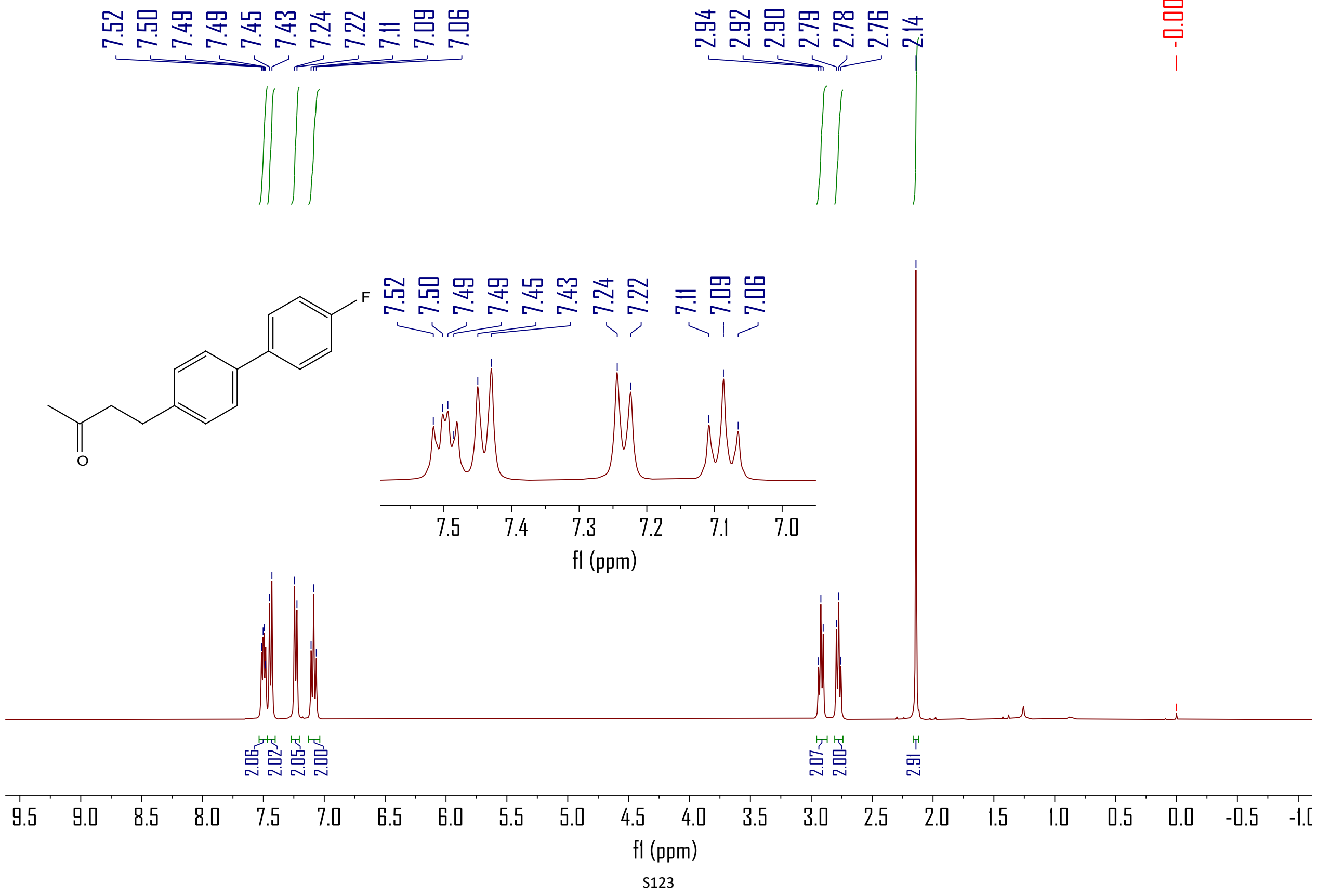


\section{${ }^{13} \mathrm{C}\left\{{ }^{1} \mathrm{H}\right\}$ NMR spectrum for compound $3 w$ following general procedure $\mathrm{B}\left(100 \mathrm{MHz}, \mathrm{CDCl}_{3}\right)$}

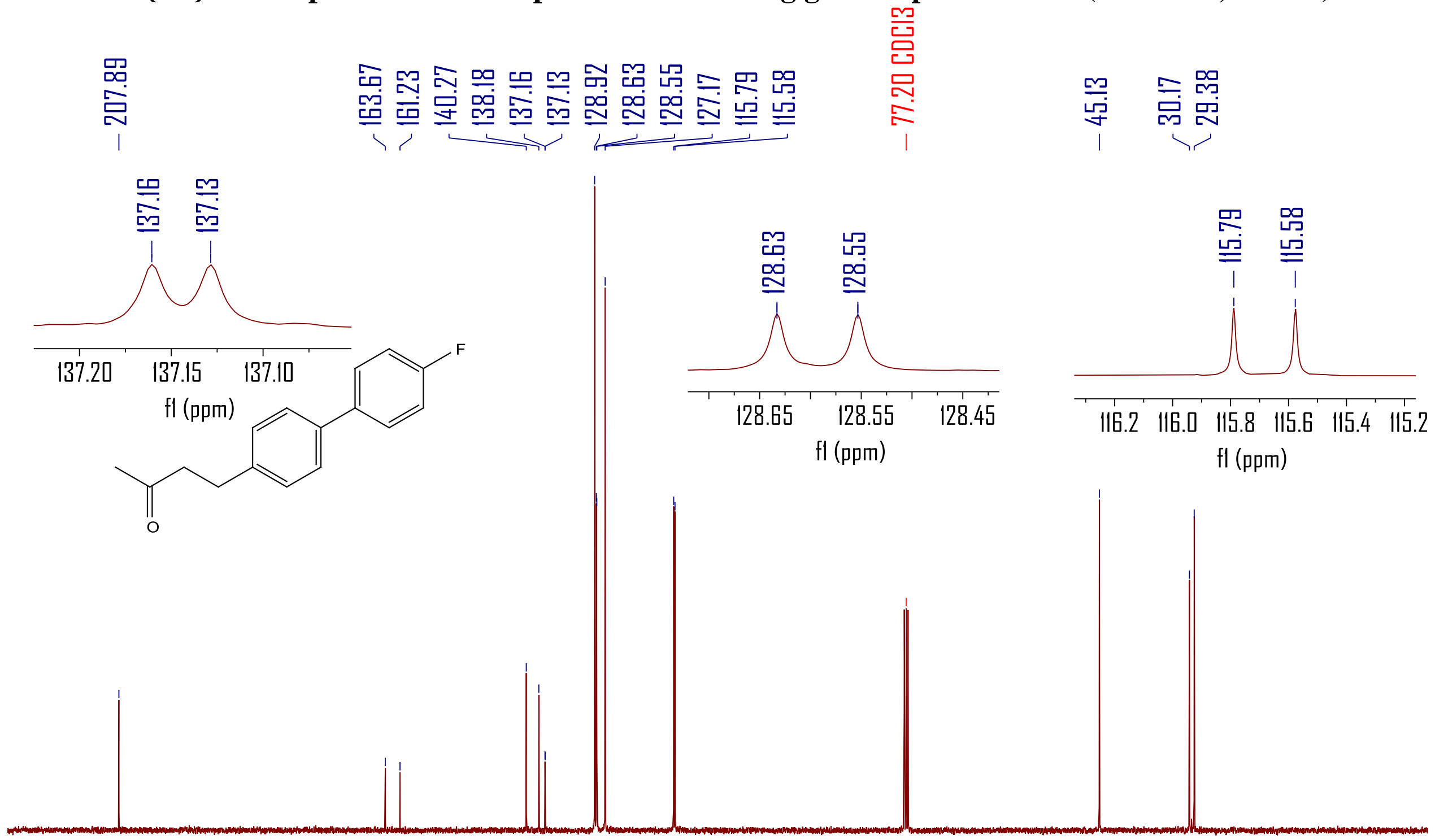

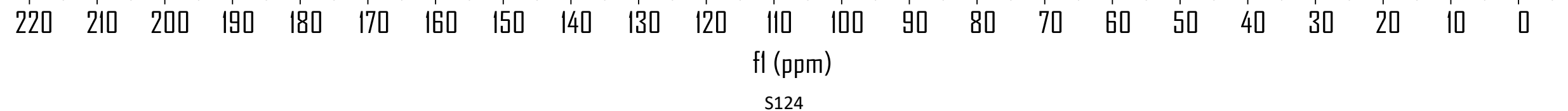


${ }^{19} \mathrm{~F}\left\{{ }^{1} \mathrm{H}\right\}$ NMR spectrum for compound 3w following general procedure $\mathrm{B}\left(376 \mathrm{MHz}, \mathrm{CDCl}_{3}\right)$

䚡
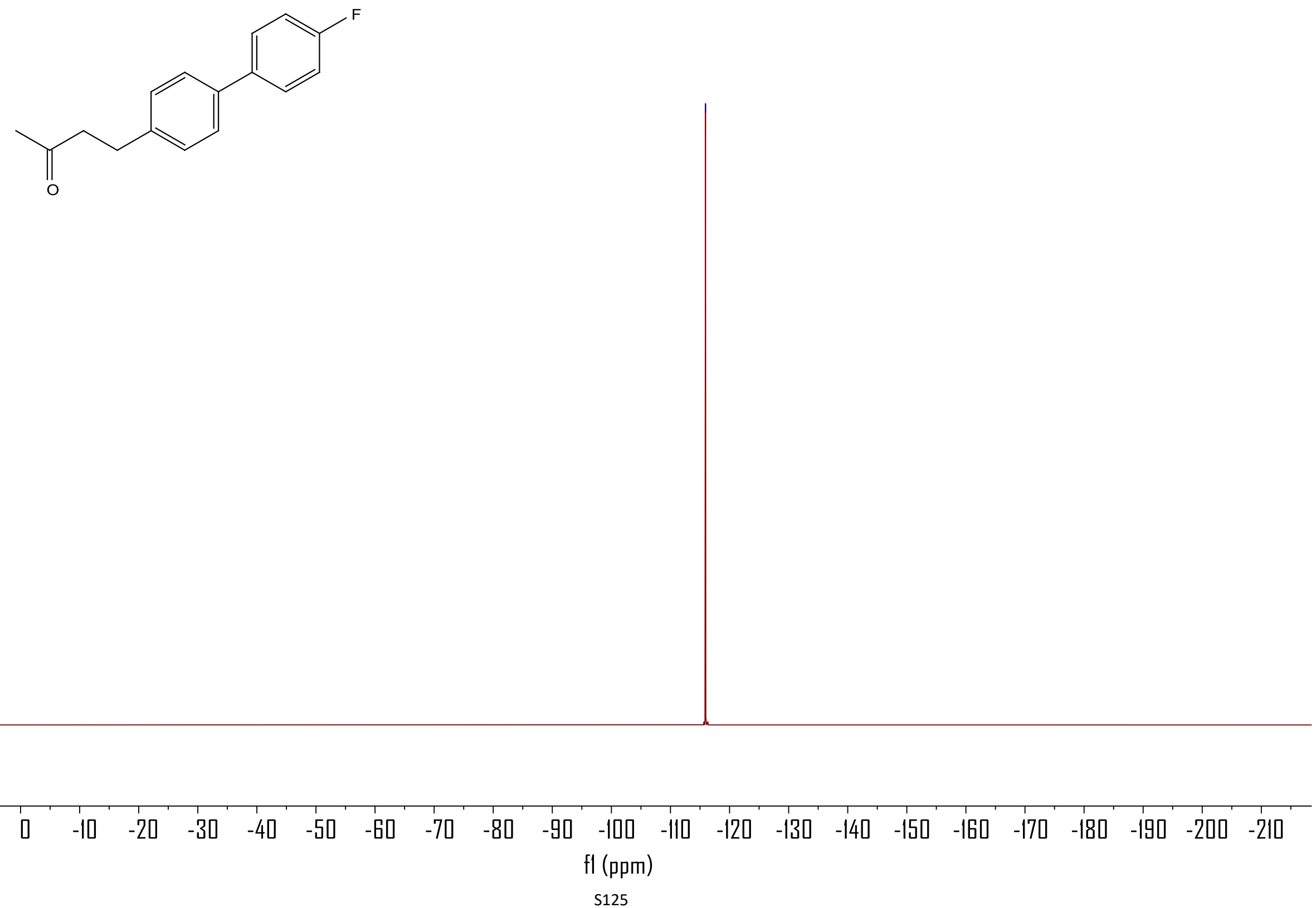
${ }^{1} \mathrm{H}$ NMR spectrum for compound $3 \mathrm{w}$ from $5.0 \mathrm{mmol}$ scale (500 $\mathrm{MHz}, \mathrm{CDCl}_{3}, \mathrm{TMS}$ )

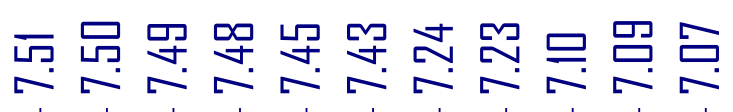

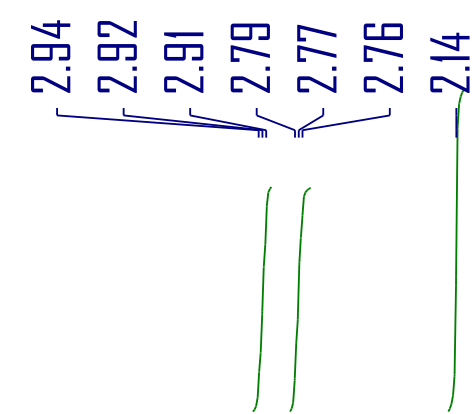

号

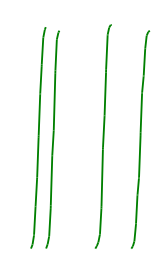

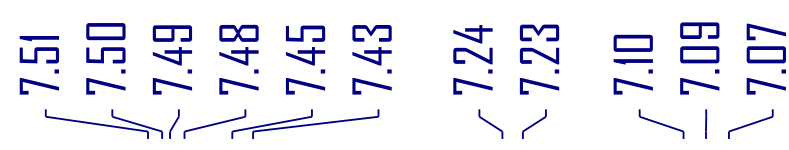
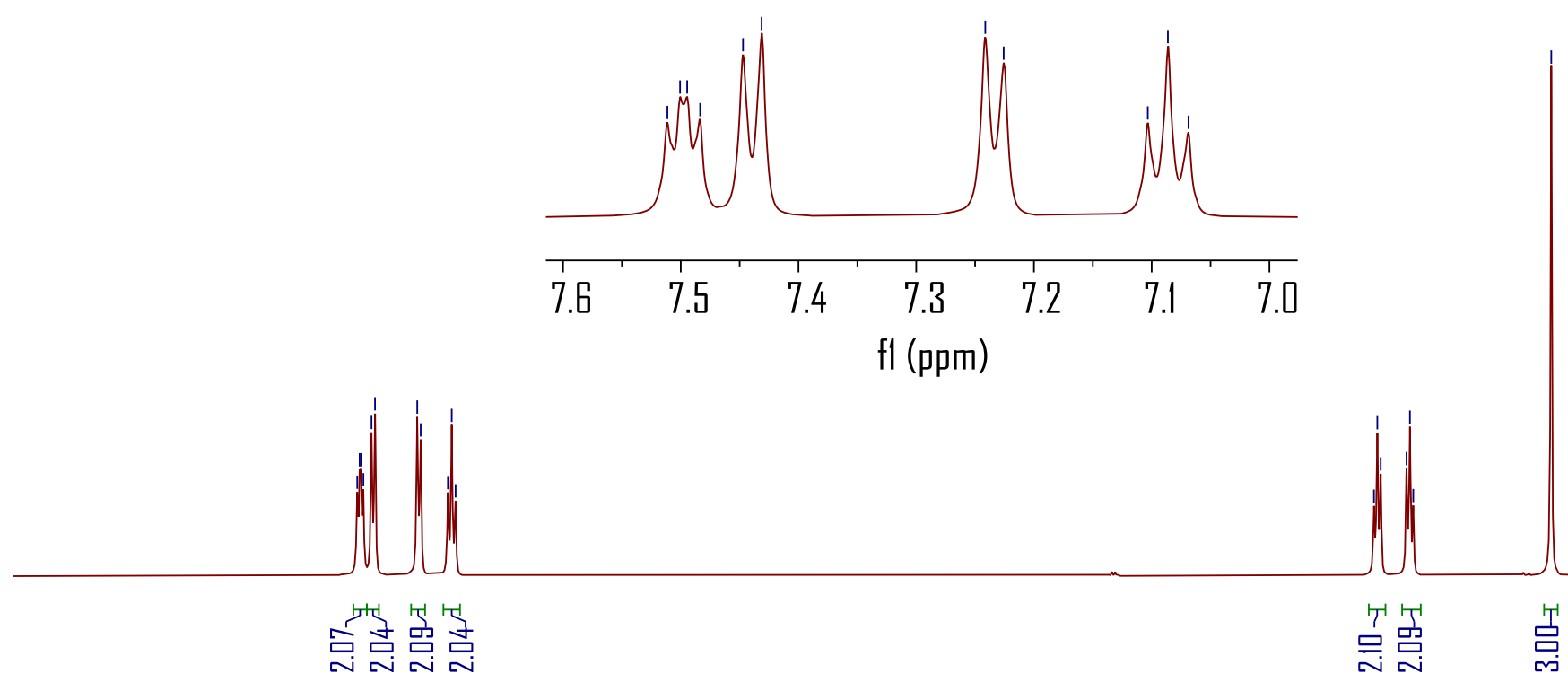

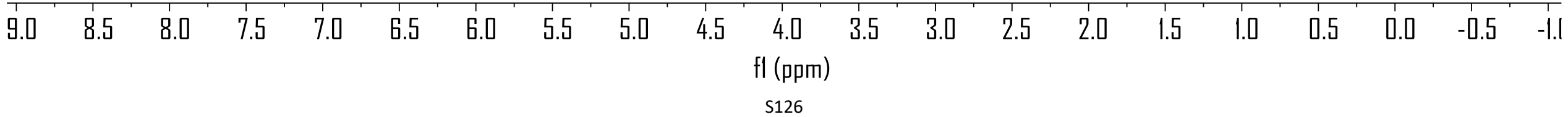




\section{${ }^{13} \mathrm{C}\left\{{ }^{1} \mathrm{H}\right\}$ NMR spectrum for compound $3 \mathrm{w}$ from $5.0 \mathrm{mmol}$ scale $\left(125 \mathrm{MHz}, \mathrm{CDCl}_{3}\right)$}

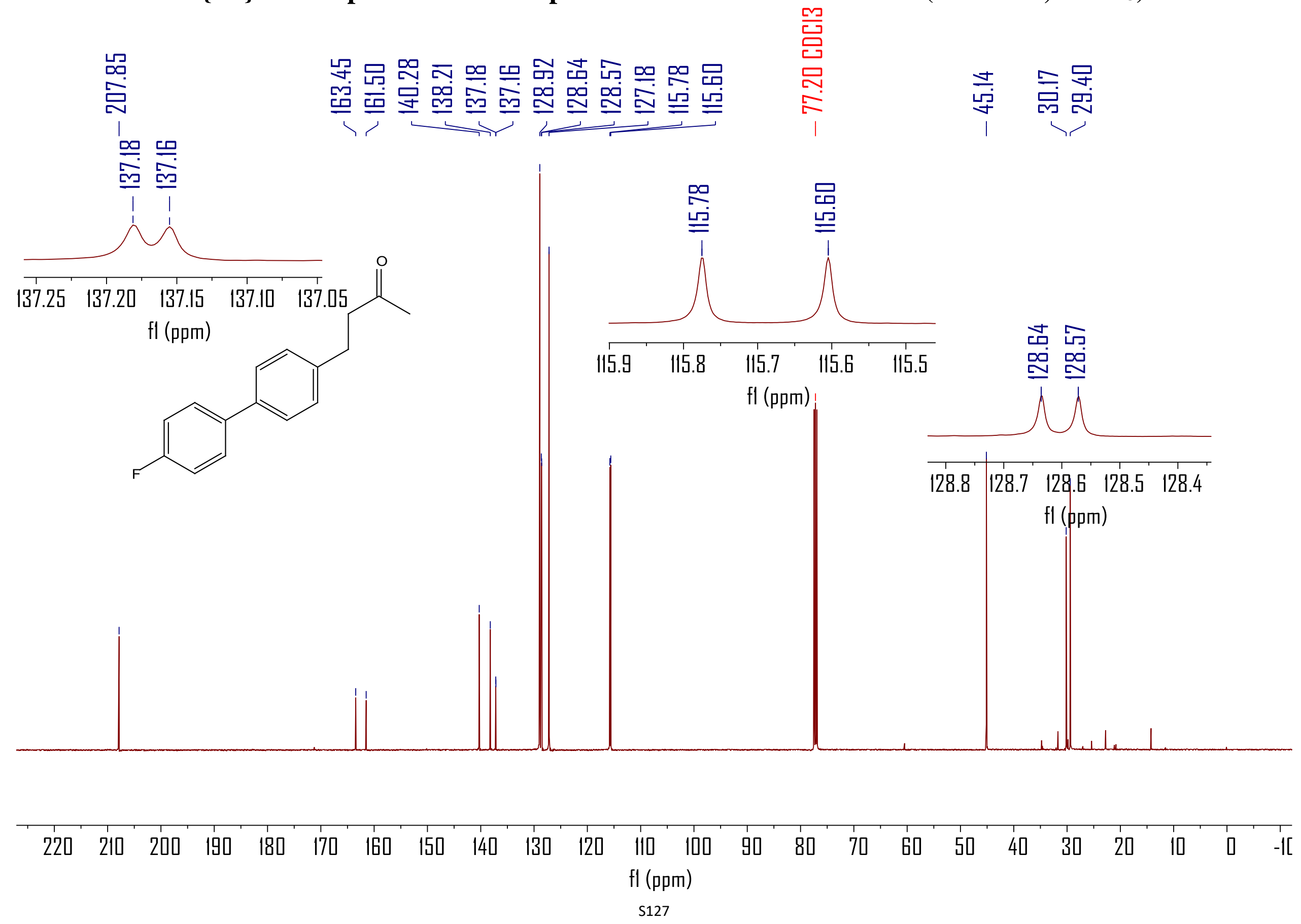


${ }^{19} \mathrm{~F}\left\{{ }^{1} \mathrm{H}\right\}$ NMR spectrum for compound 3w from $5.0 \mathrm{mmol}$ scale $\left(376 \mathrm{MHz}, \mathrm{CDCl}_{3}\right)$

苟
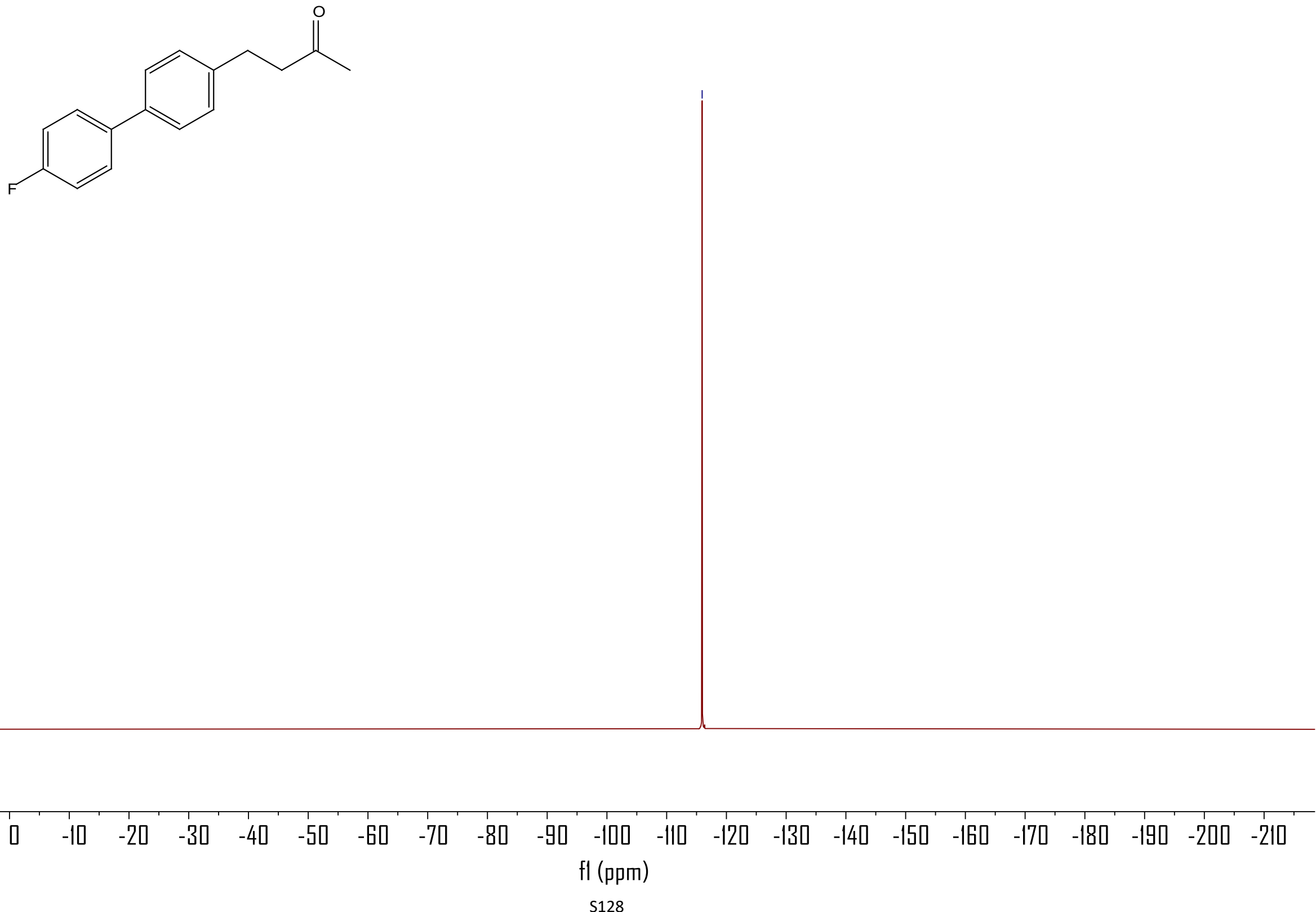


\section{${ }^{1} \mathrm{H}$ NMR spectrum for compound 3x (500 $\mathrm{MHz}, \mathrm{CDCl}_{3}$, TMS)}

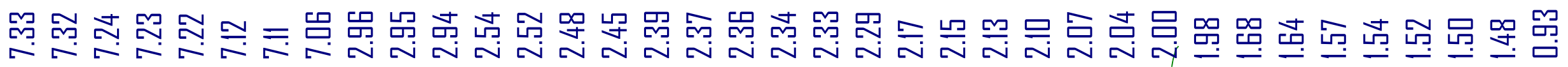

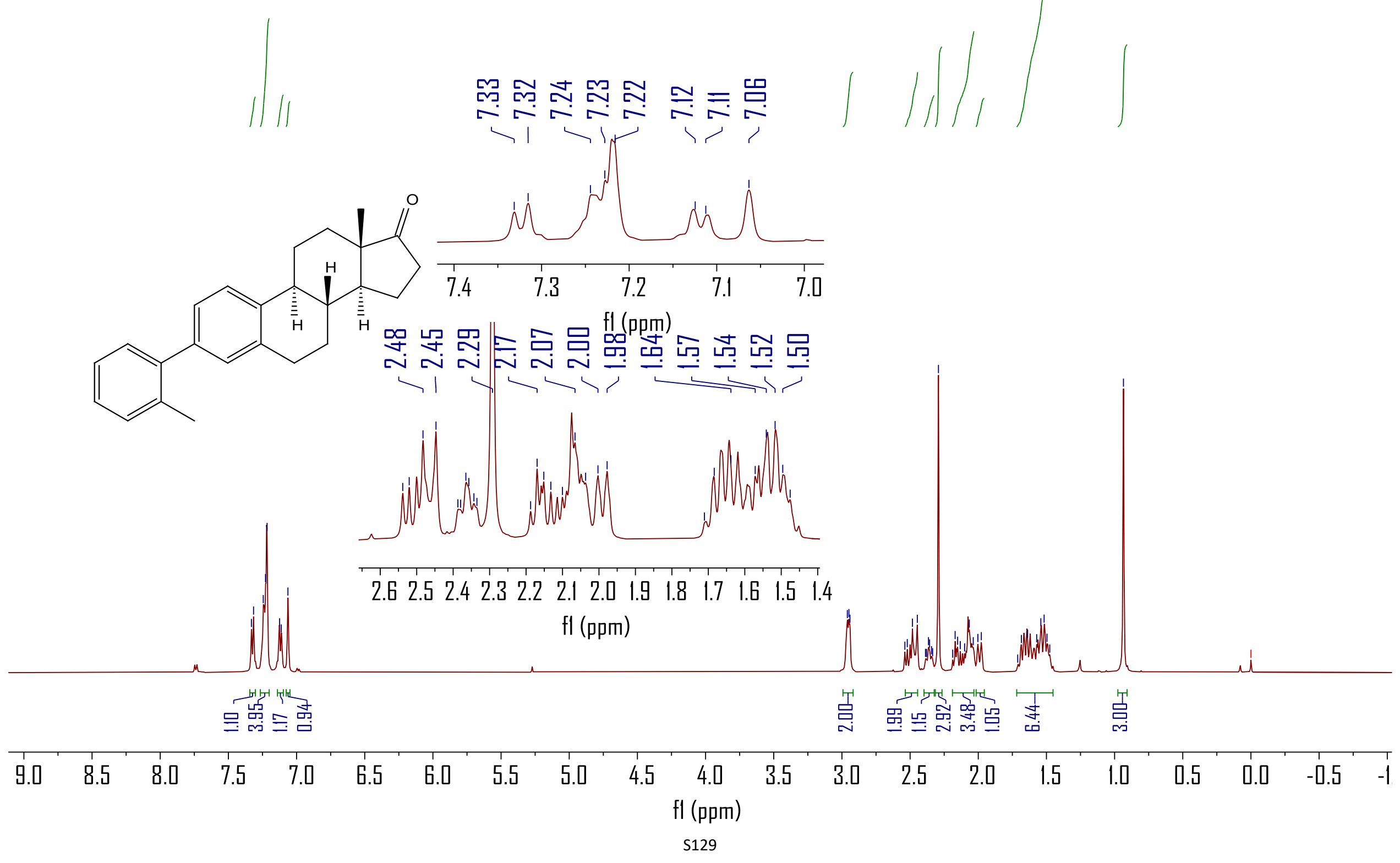




\section{${ }^{13} \mathrm{C}\left\{{ }^{1} \mathrm{H}\right\}$ NMR spectrum for compound $3 x\left(125 \mathrm{MHz}, \mathrm{CDCl}_{3}\right)$}

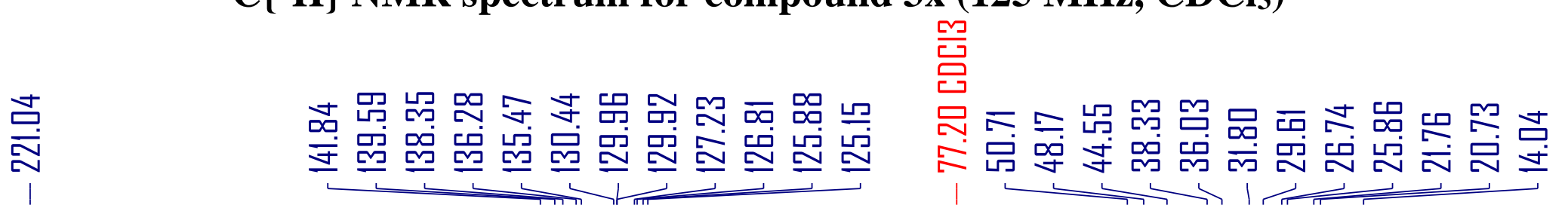
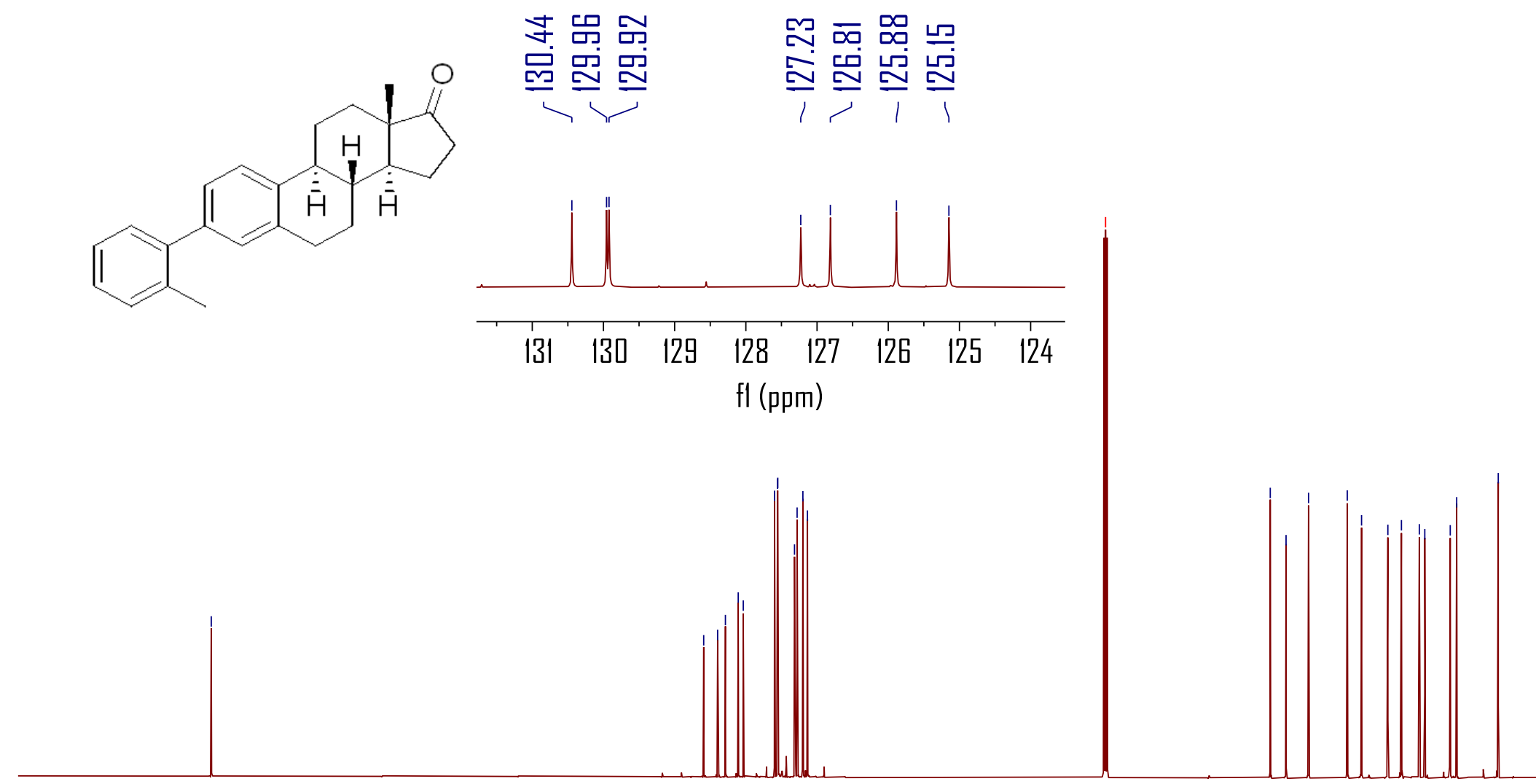

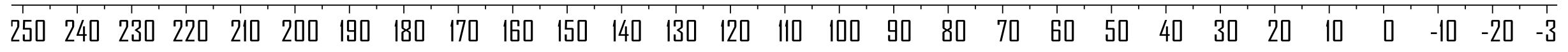
$\mathrm{fl}(\mathrm{ppm})$ 


\section{${ }^{1} \mathrm{H}$ NMR spectrum for compound $3 \mathrm{y}\left(400 \mathrm{MHz}, \mathrm{CDCl}_{3}\right)$}

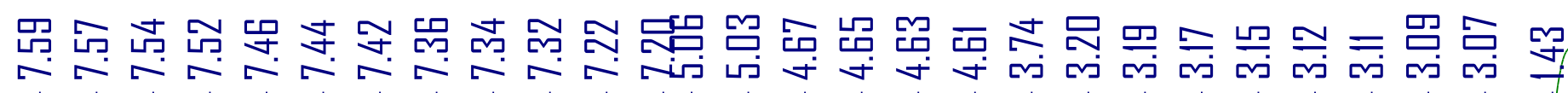

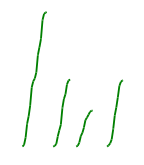

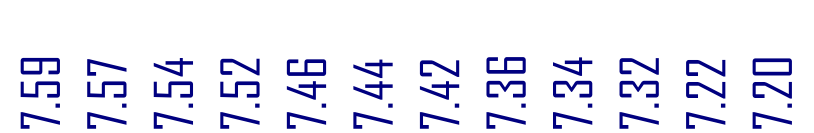

吕 몸

品品䏰品

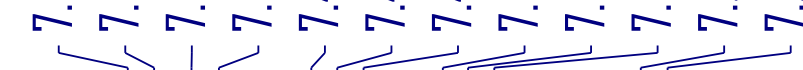
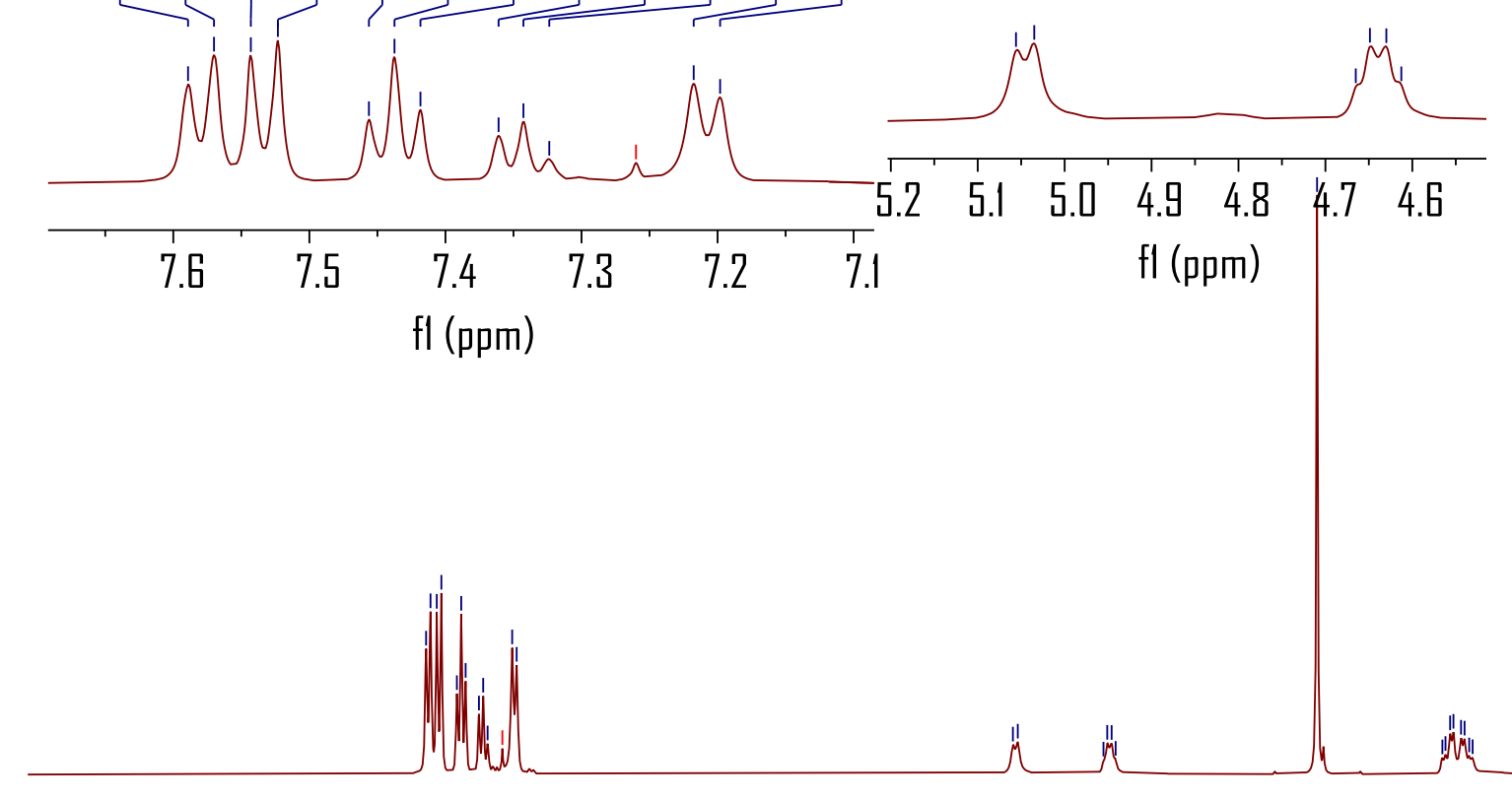

亭总言骂

$\mathrm{fl}(\mathrm{ppm})$
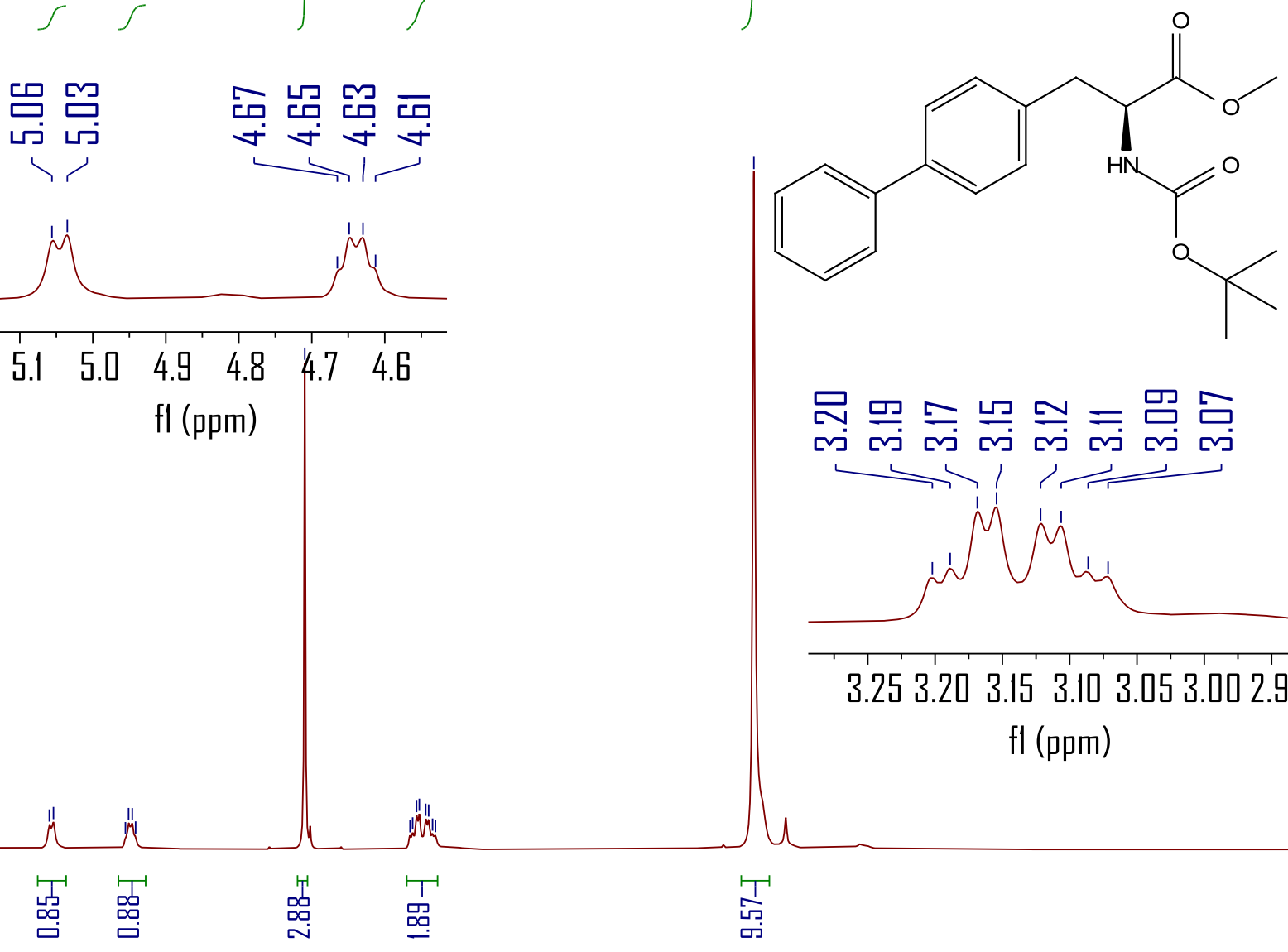

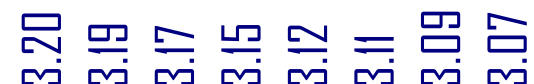

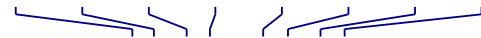

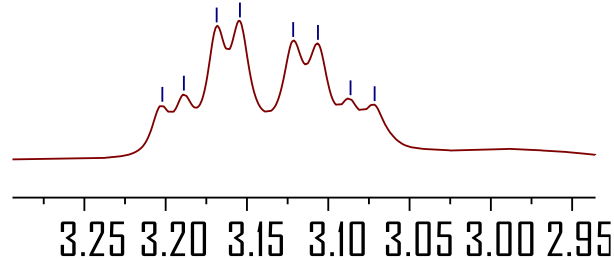
$\mathrm{fl}(\mathrm{ppm})$

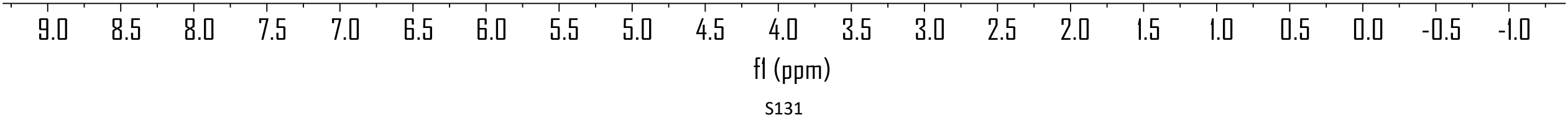




\section{${ }^{13} \mathrm{C}\left\{{ }^{1} \mathrm{H}\right\}$ NMR spectrum for compound $3 \mathrm{y}\left(100 \mathrm{MHz}, \mathrm{CDCl}_{3}\right)$}

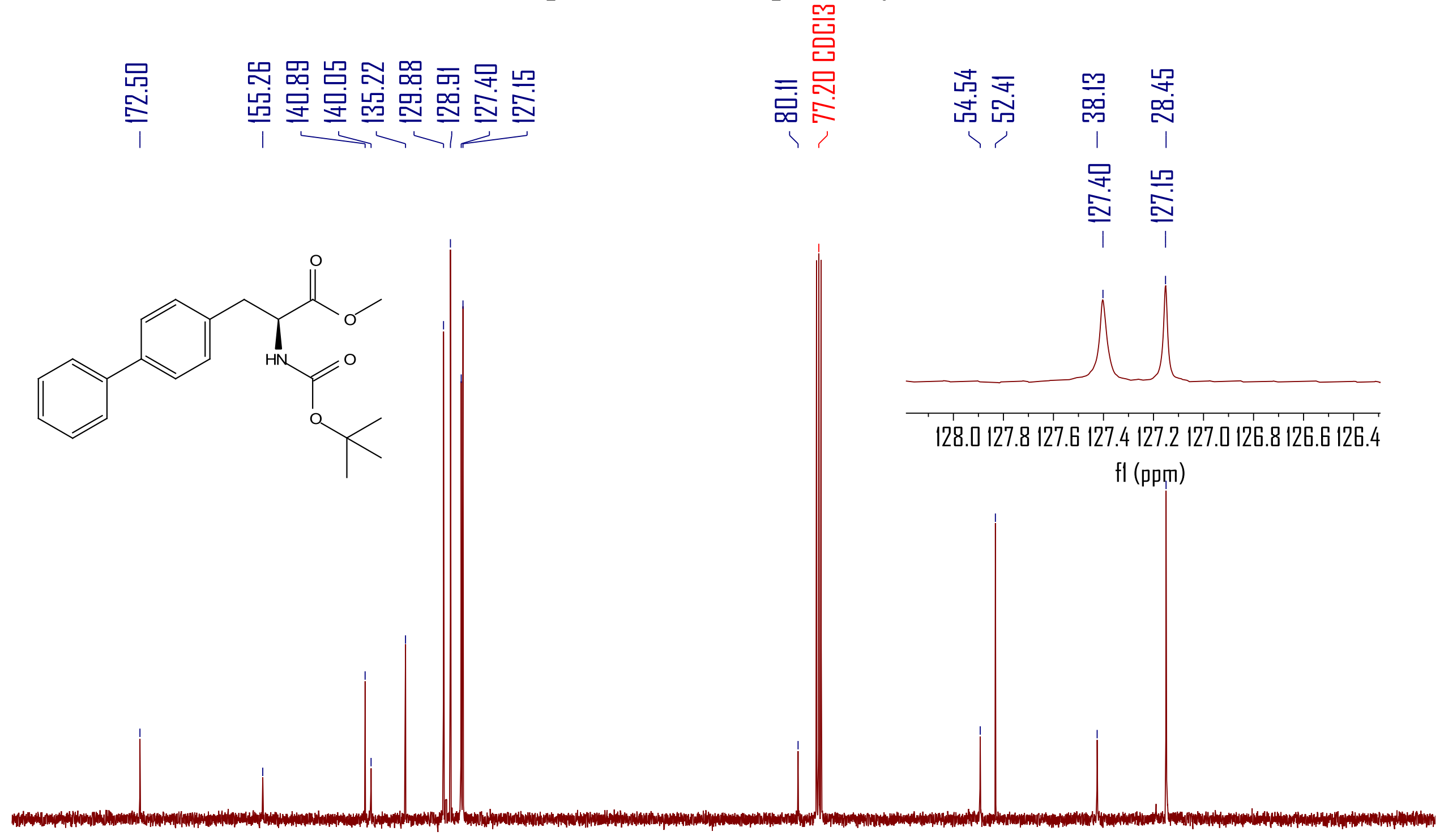

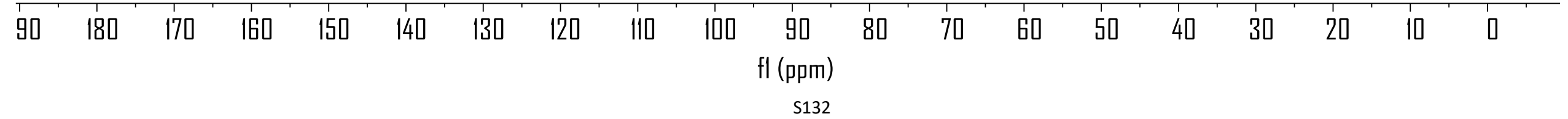




\section{${ }^{1}$ H NMR spectrum for compound 3z (500 MHz, DMSO-d6)}

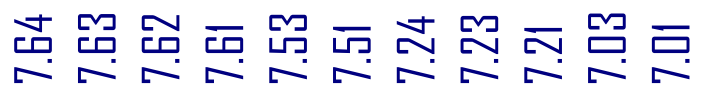

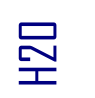

号

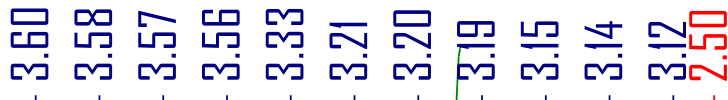
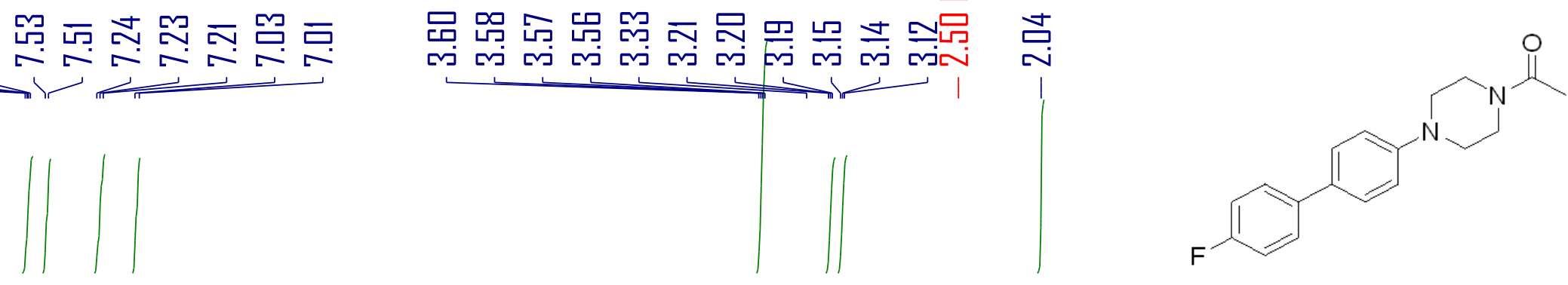

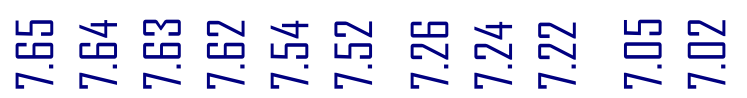

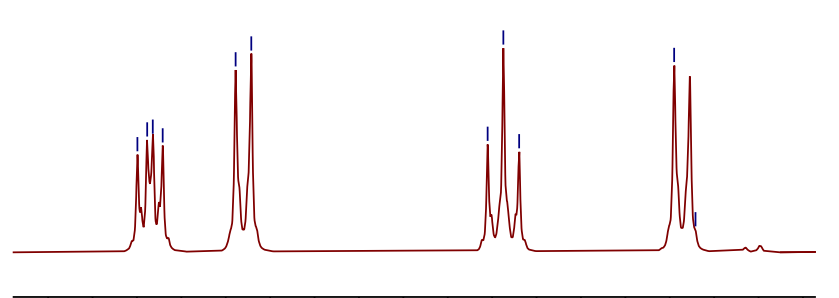

$\begin{array}{ccccccccc}7.7 & 7.6 & 7.5 & 7.4 & 7.3 & 7.2 & 7.1 & 7.0 & 6.9 \\ \mathrm{fl}(\mathrm{ppm}) & & & & \end{array}$
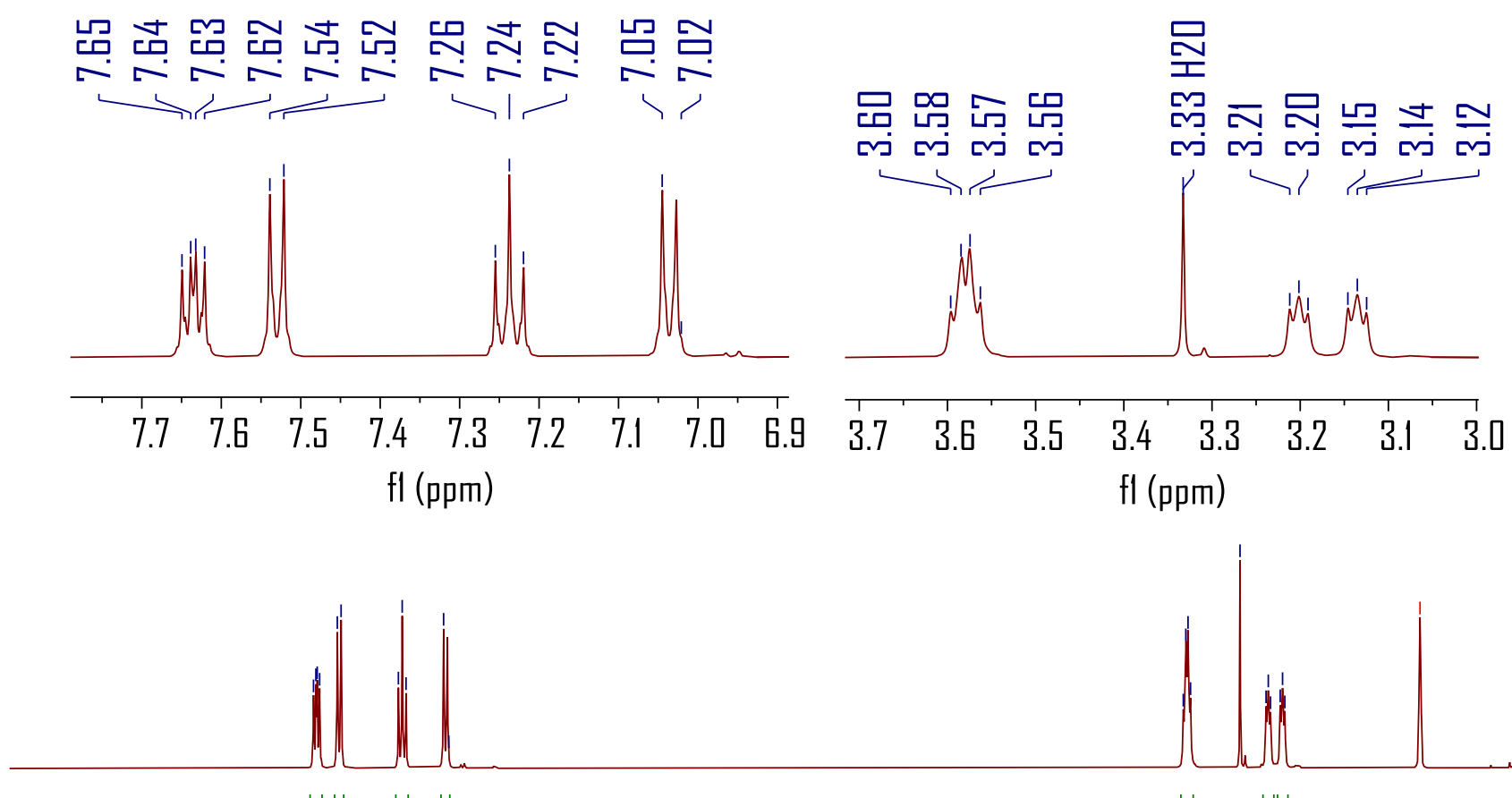

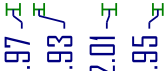

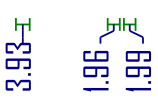

岁

\begin{tabular}{llllllllllllllllllll}
\hline 9.0 & 8.5 & 8.0 & 7.5 & 7.0 & 6.5 & 6.0 & 5.5 & 5.0 & 4.5 & 4.0 & 3.5 & 3.0 & 2.5 & 2.0 & 1.5 & 1.0 & 0.5 & 0.0 & -0.5
\end{tabular}




\section{${ }^{13} \mathrm{C}\left\{{ }^{1} \mathrm{H}\right\}$ NMR spectrum for compound 3z (DMSO-d $6,125 \mathrm{MHz}$ )}

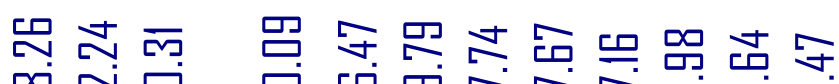

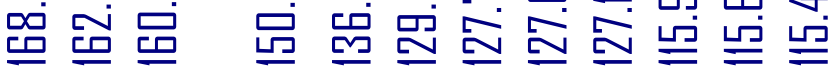
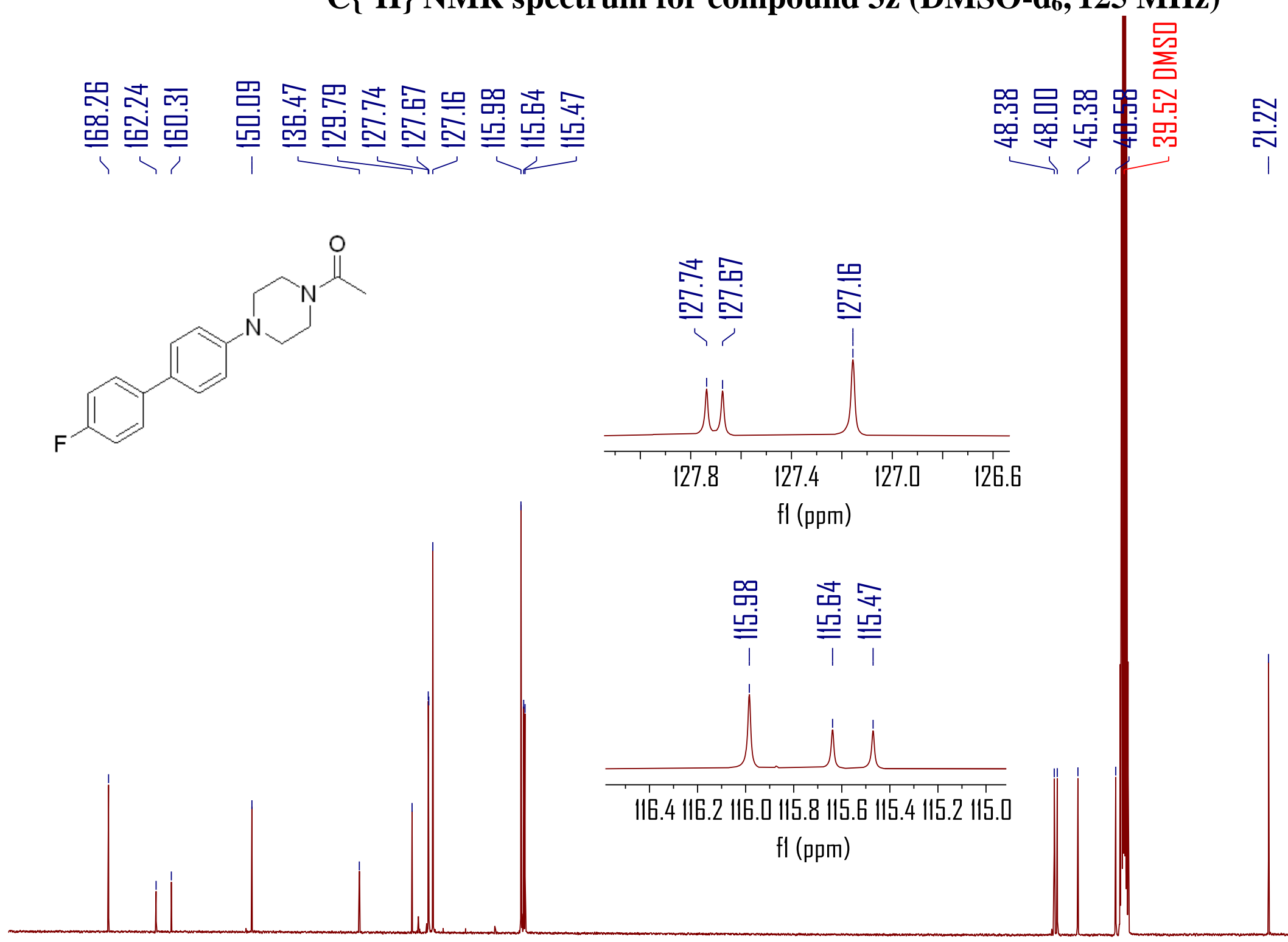


\section{${ }^{19} \mathrm{~F}\left\{{ }^{1} \mathrm{H}\right\}$ NMR spectrum for compound 3z (DMSO-d6, $376 \mathrm{MHz}$ )}

$$
\text { 号 }
$$
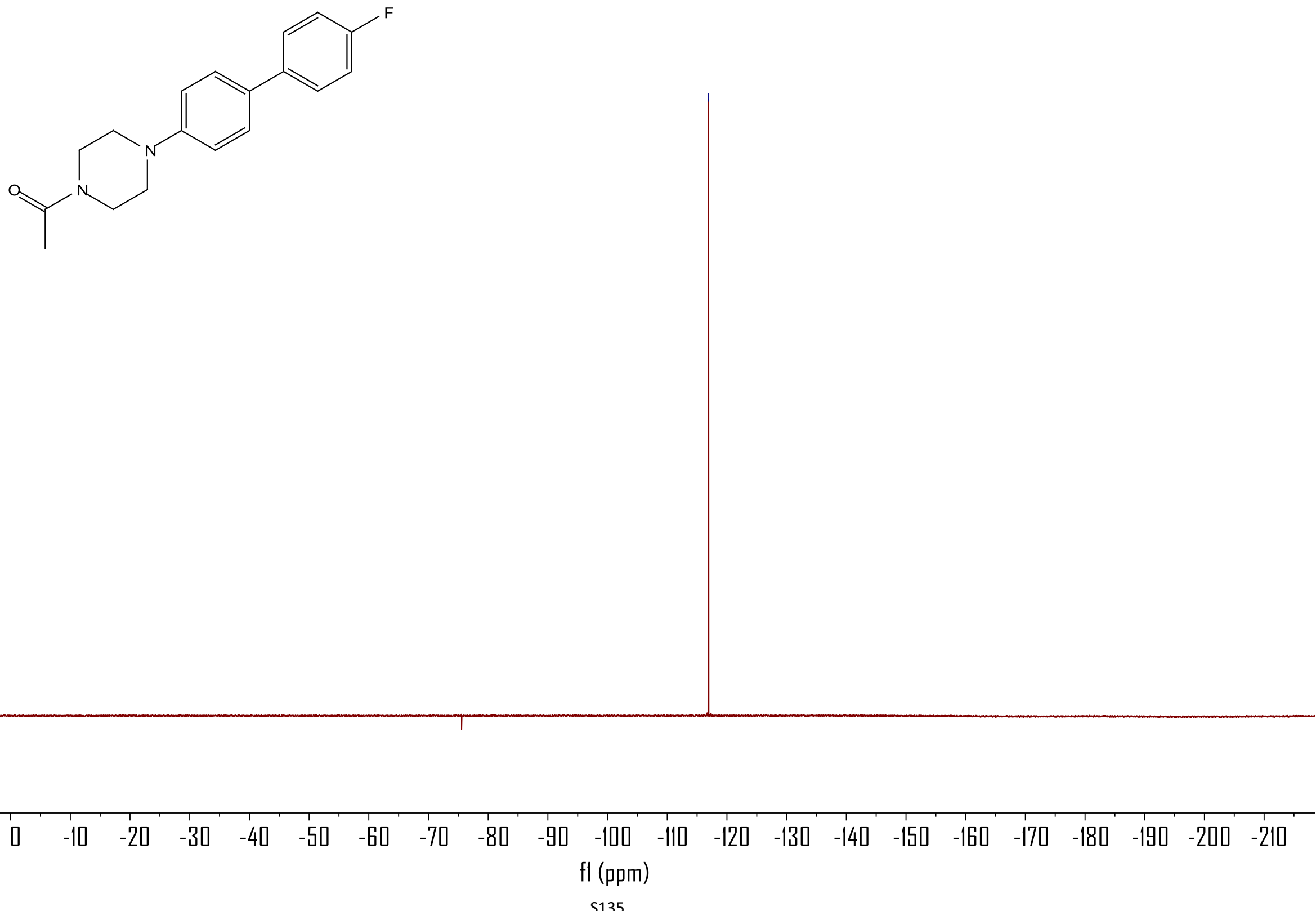


\section{${ }^{1} \mathrm{H}$ NMR spectrum for compound 3aa (500 $\left.\mathrm{MHz}, \mathrm{CDCl}_{3}\right)$}

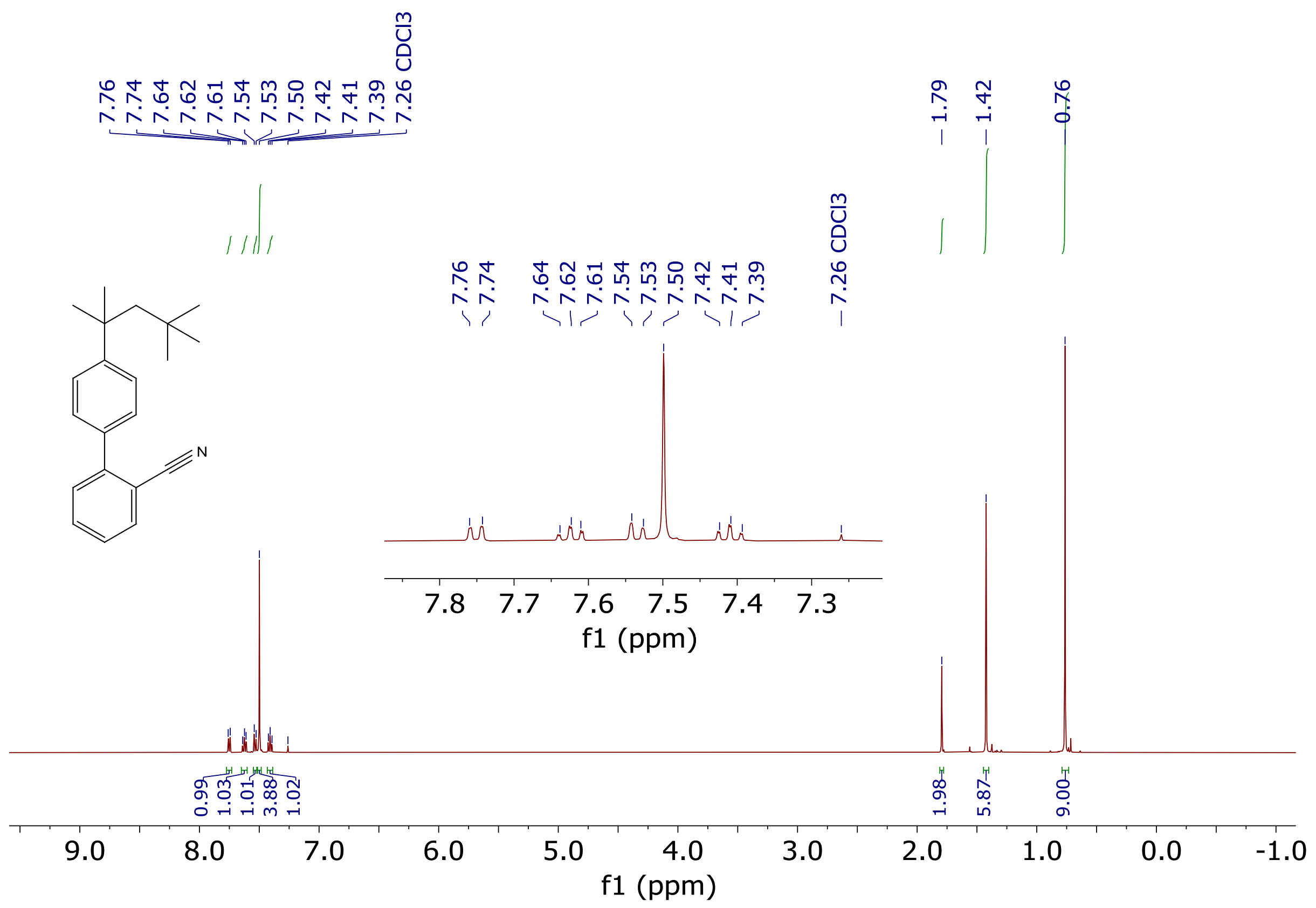




\section{${ }^{13} \mathrm{C}\left\{{ }^{1} \mathrm{H}\right\}$ NMR spectrum for compound 3aa (125 $\left.\mathrm{MHz}, \mathrm{CDCl}_{3}\right)$}

ન

峲

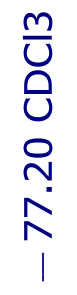

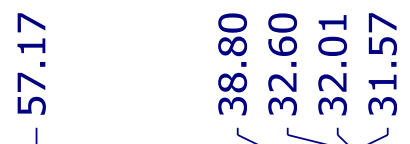
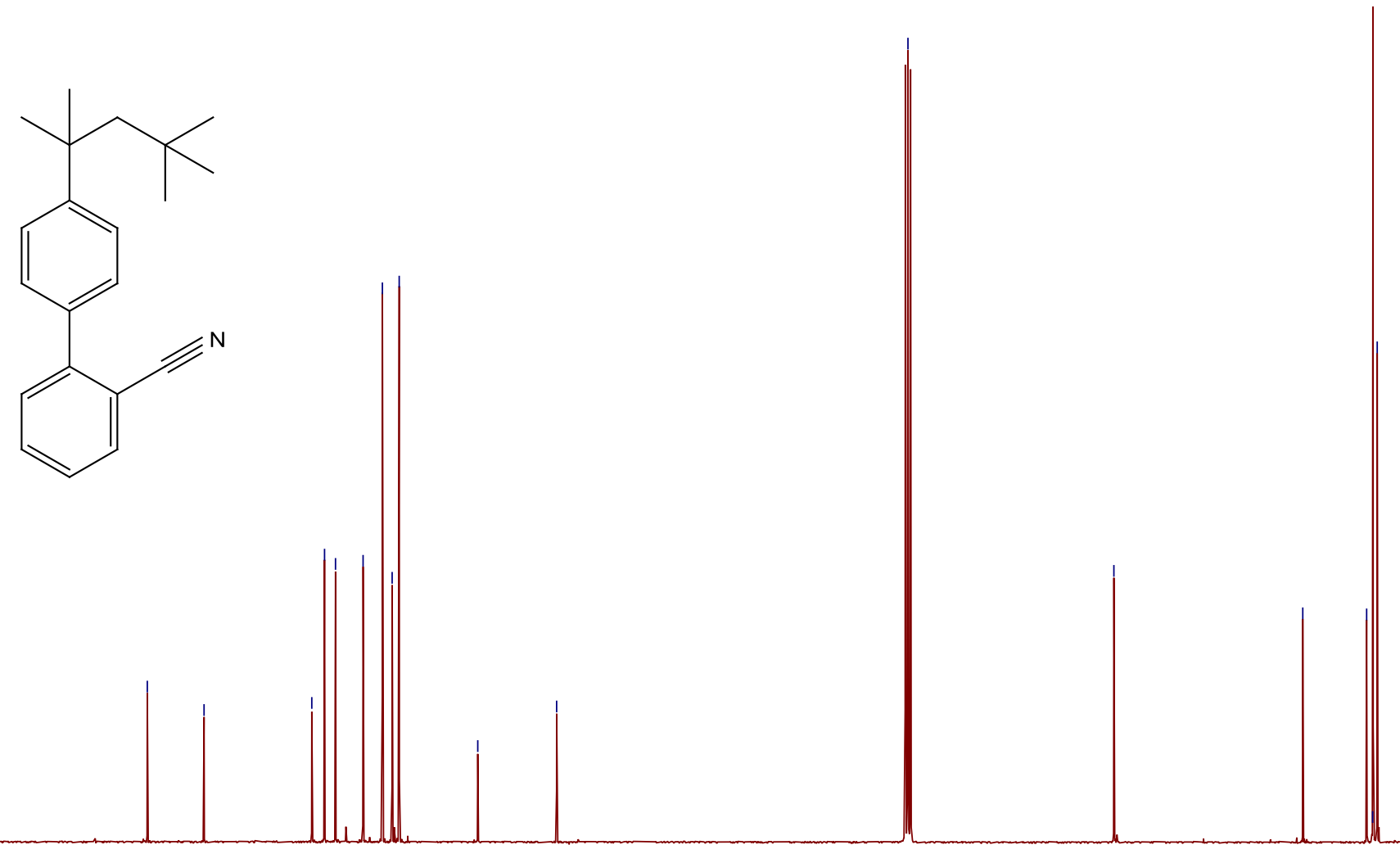

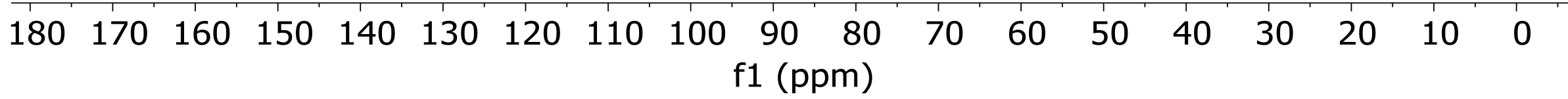




\section{${ }^{1} \mathrm{H}$ NMR spectrum for compound $3 \mathrm{ab}\left(500 \mathrm{MHz}, \mathrm{CDCl}_{3}\right)$}

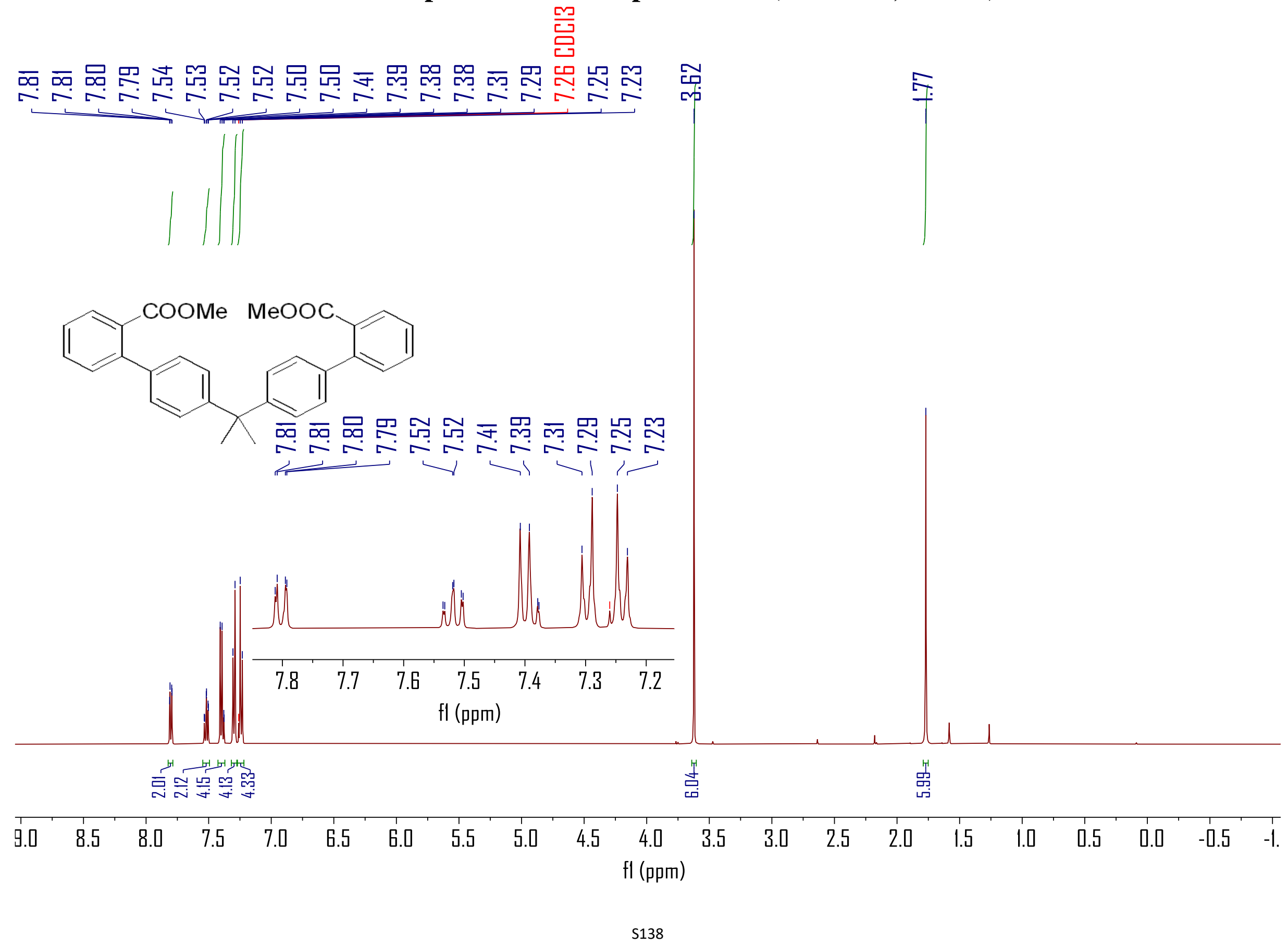




\section{${ }^{13} \mathrm{C}\left\{{ }^{1} \mathrm{H}\right\}$ NMR spectrum for compound $3 \mathrm{ab}\left(125 \mathrm{MHz}, \mathrm{CDCl}_{3}\right)$}

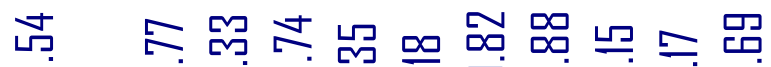

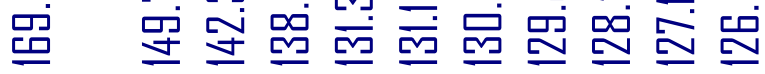
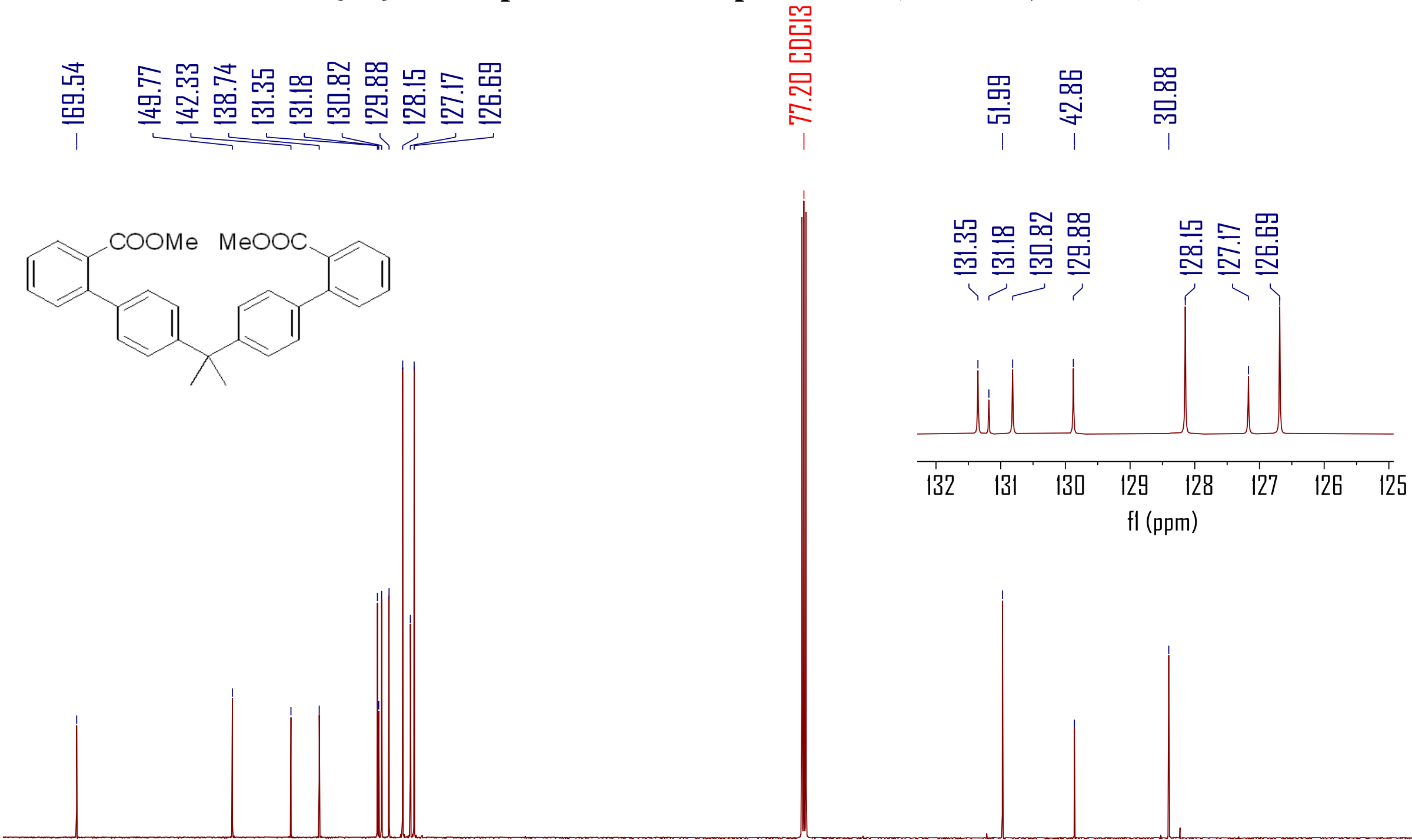

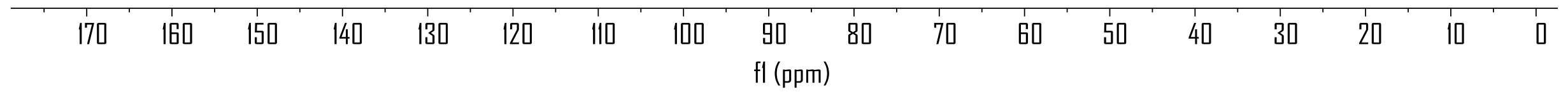




\section{${ }^{1} \mathrm{H}$ NMR spectrum for compound $3 \mathrm{ac}\left(500 \mathrm{MHz}, \mathrm{CDCl}_{3}\right)$}

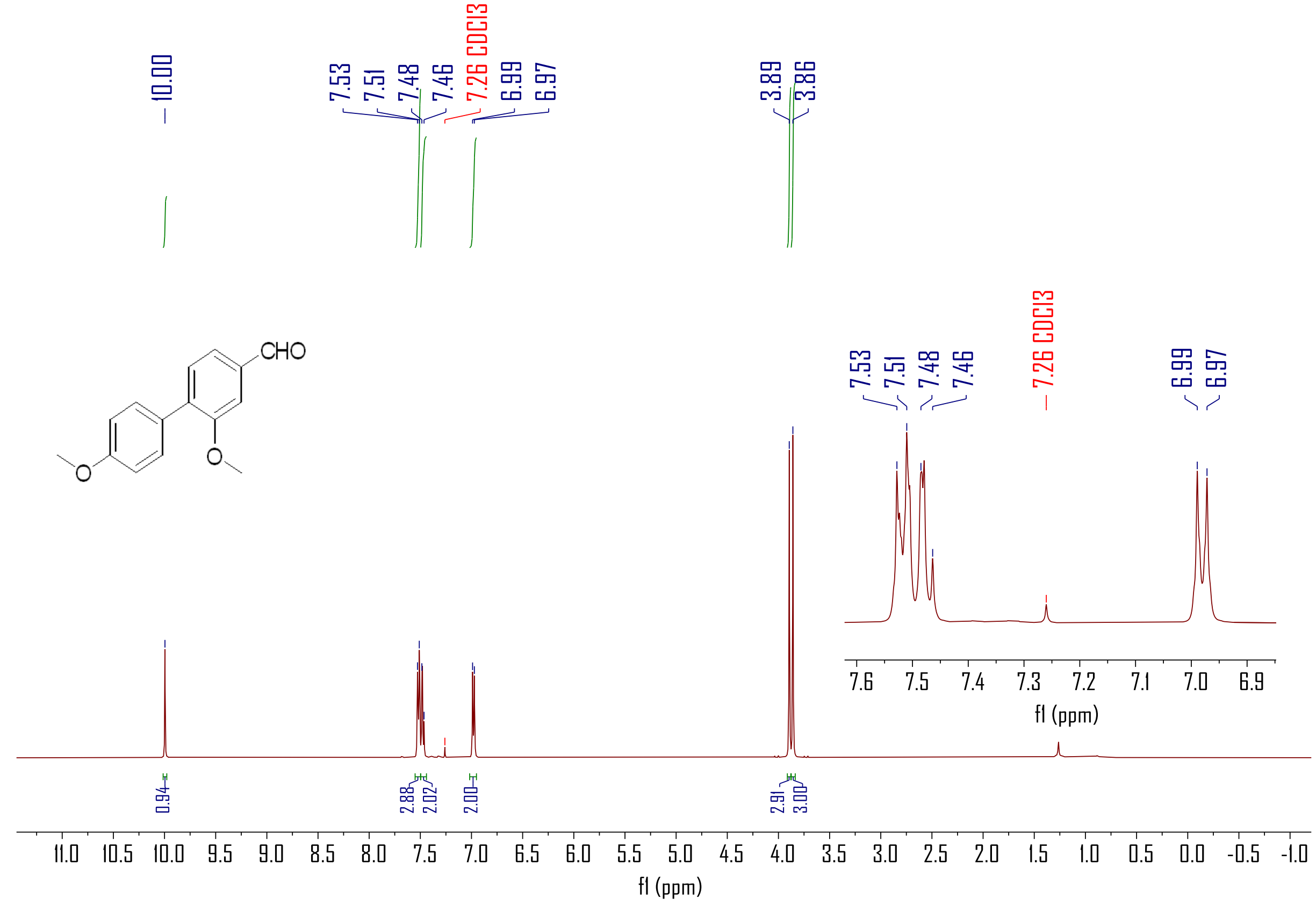




\section{${ }^{13} \mathrm{C}\left\{{ }^{1} \mathrm{H}\right\}$ NMR spectrum for compound 3ac $\left(125 \mathrm{MHz}, \mathrm{CDCl}_{3}\right)$}
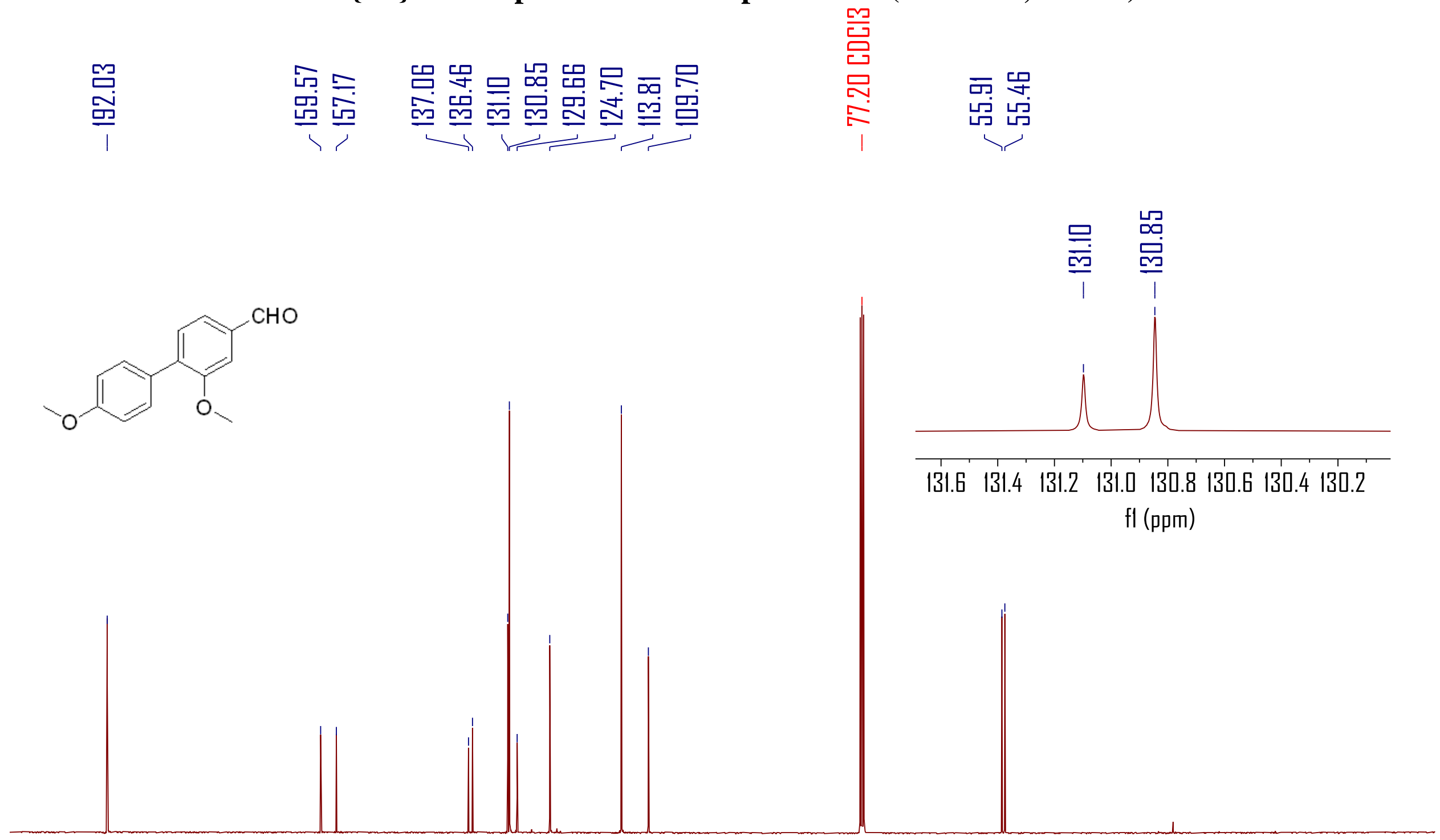

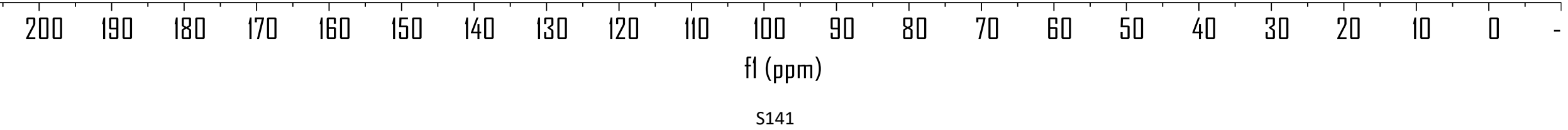




\section{${ }^{1} \mathrm{H}$ NMR spectrum for compound $3 \mathrm{ad}\left(500 \mathrm{MHz}, \mathrm{CDCl}_{3}\right)$}

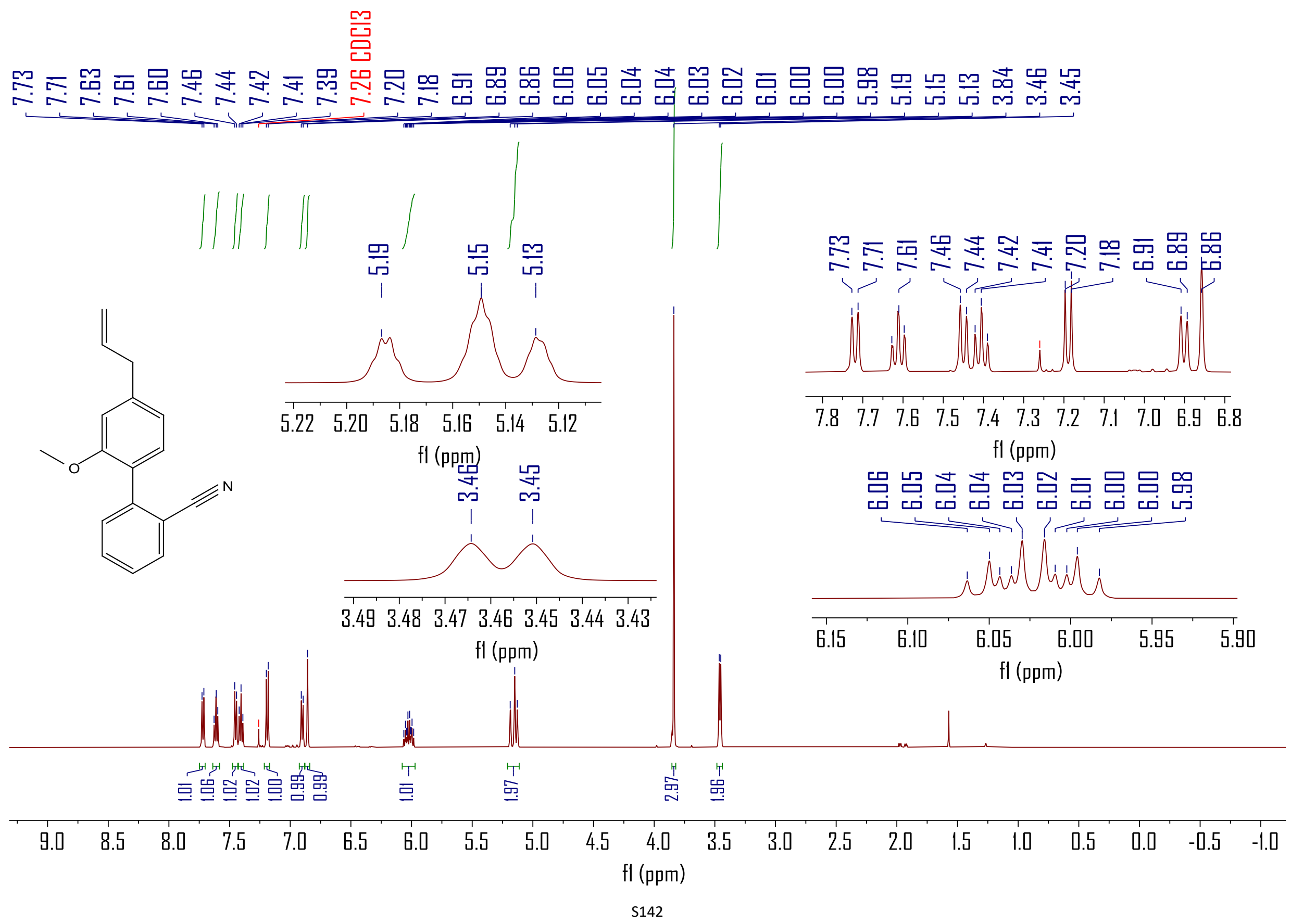




\section{${ }^{13} \mathrm{C}\left\{{ }^{1} \mathrm{H}\right\}$ NMR spectrum for compound 3ad (125 $\left.\mathrm{MHz}, \mathrm{CDCl}_{3}\right)$}

\begin{tabular}{|c|c|c|}
\hline 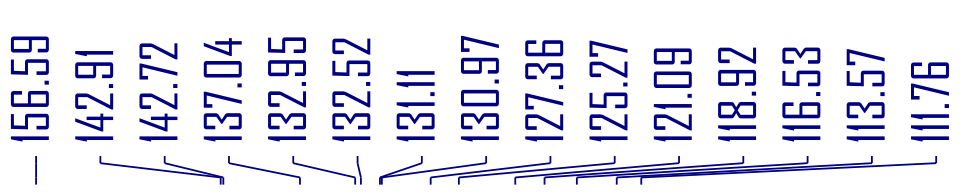 & 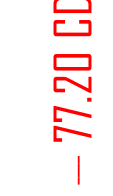 & 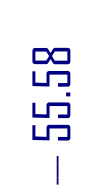 \\
\hline
\end{tabular}
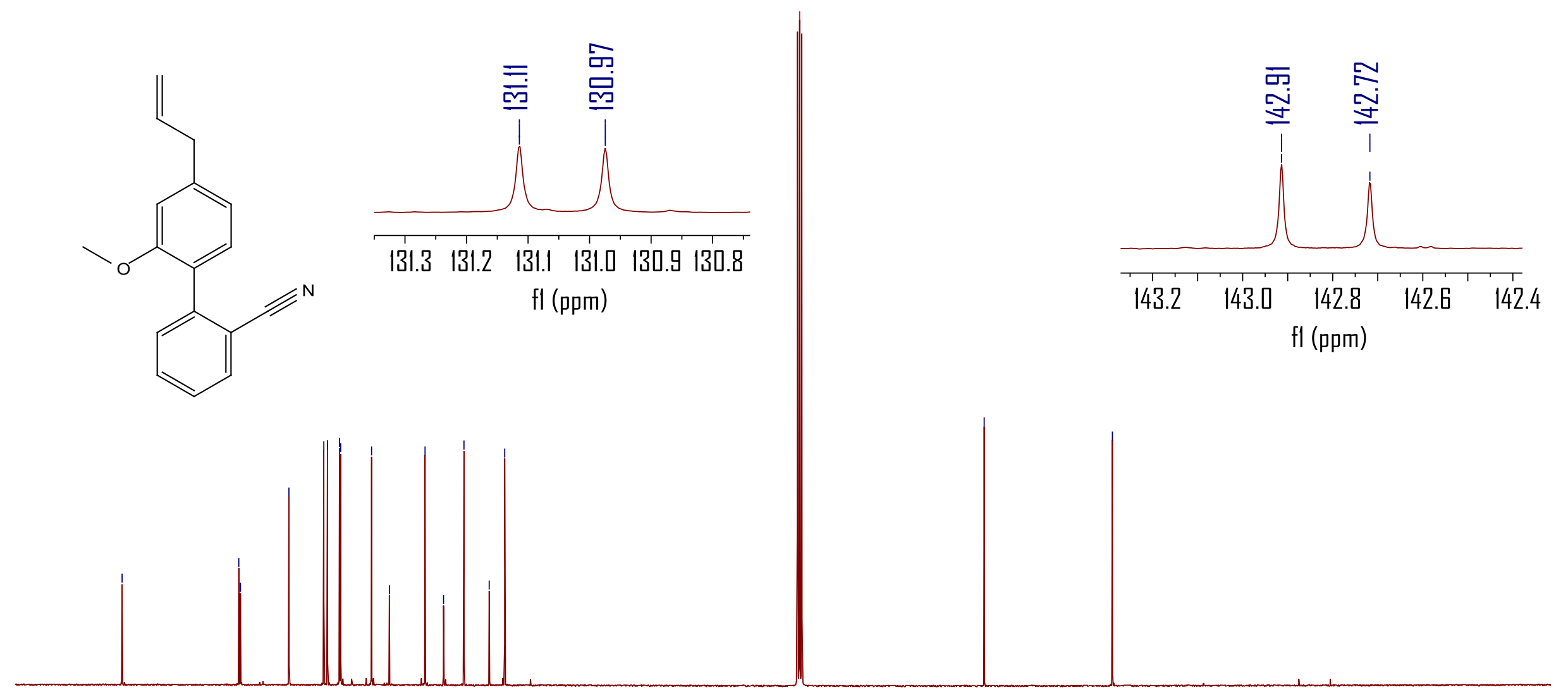

字

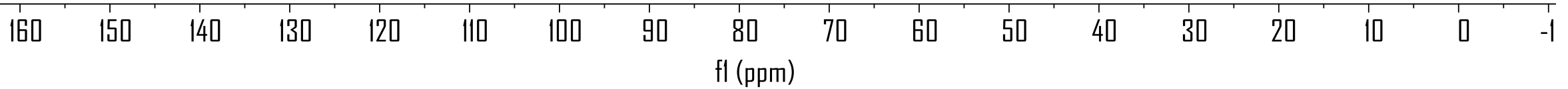


${ }^{1} \mathrm{H}$ NMR spectrum for compound 3ae (500 $\left.\mathrm{MHz}, \mathrm{CDCl}_{3}\right)$

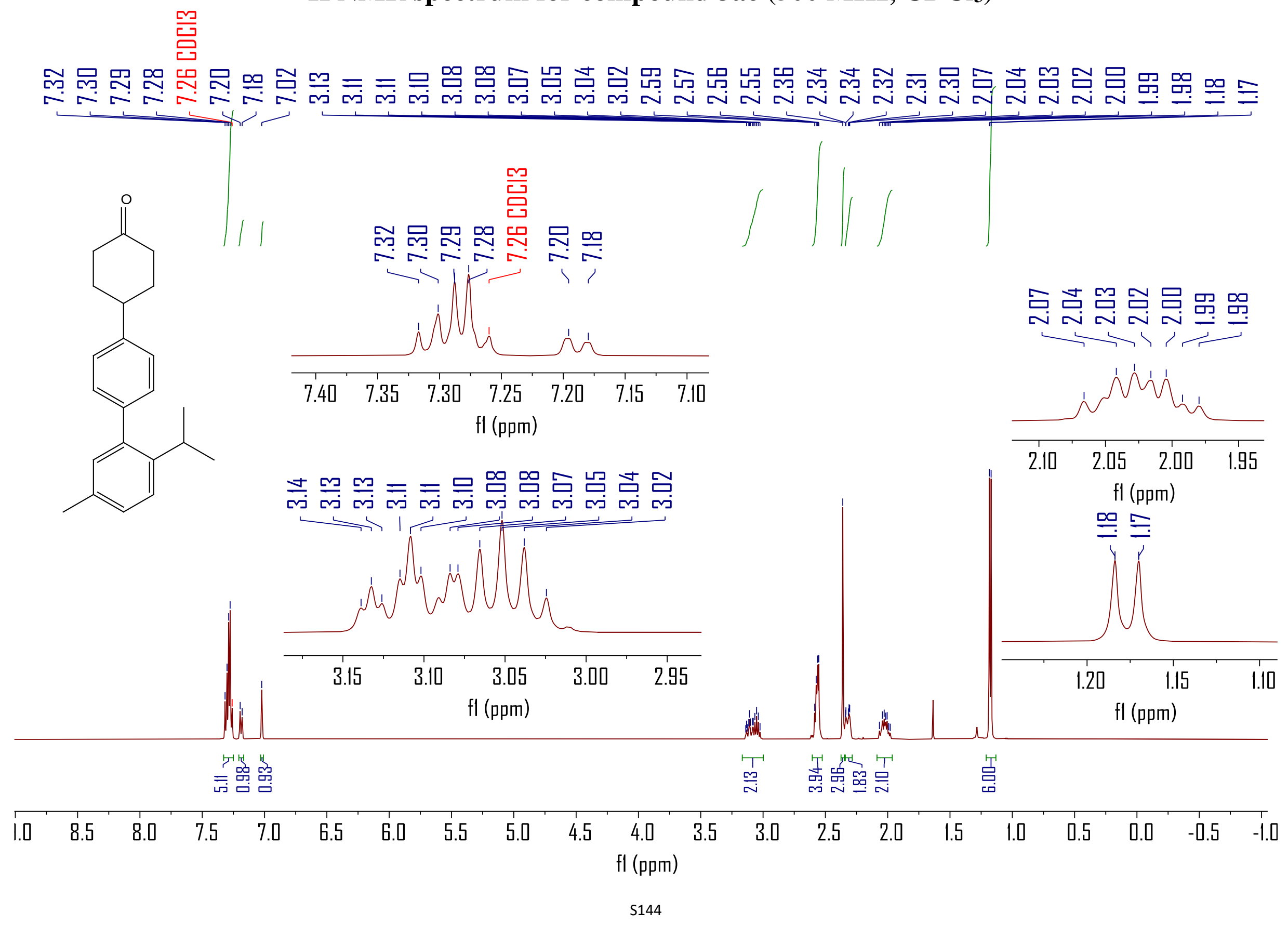


${ }^{13} \mathrm{C}\left\{{ }^{1} \mathrm{H}\right\}$ NMR spectrum for compound 3ae (125 $\left.\mathrm{MHz}, \mathrm{CDCl}_{3}\right)$

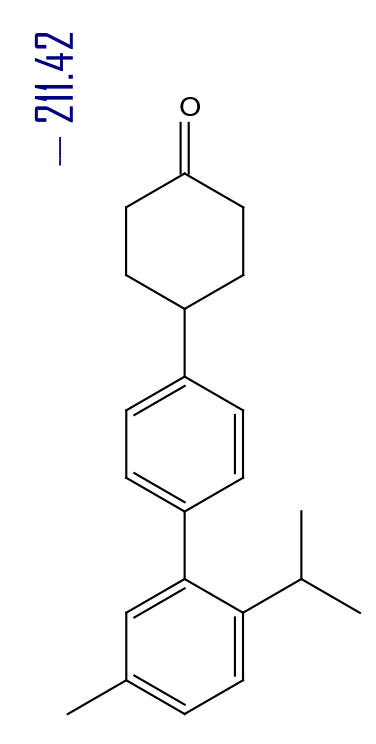

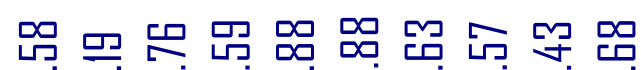

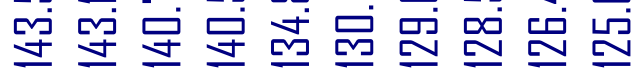

믈

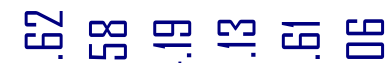

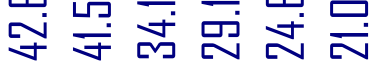
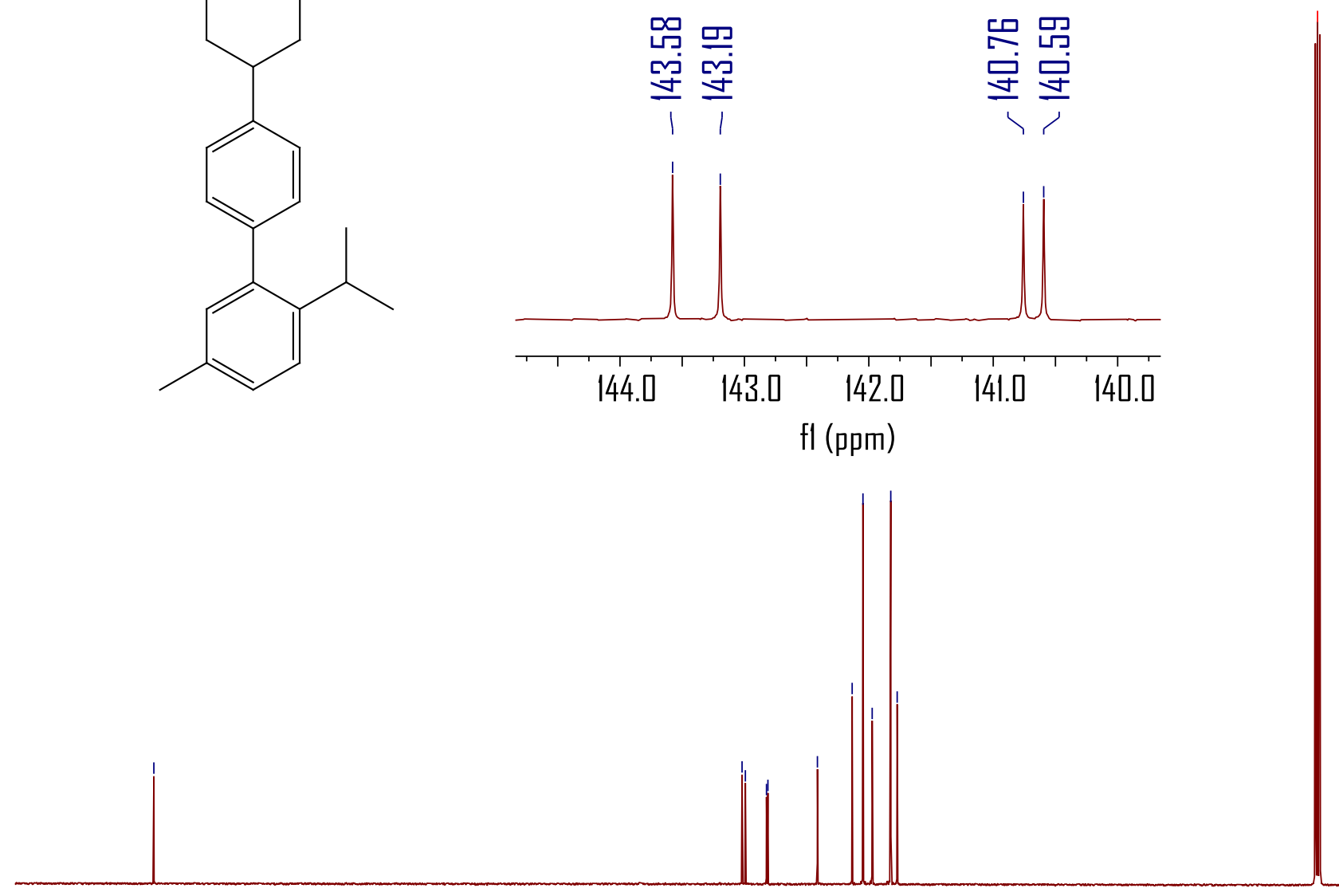

$\begin{array}{llllll}220 & 210 & 200 & 190 & 180 & 170\end{array}$

$160 \quad 150$

$150 \quad 140$

$40 \quad 130$

$130 \quad 120$

$\mathrm{fl}(\mathrm{ppm})$

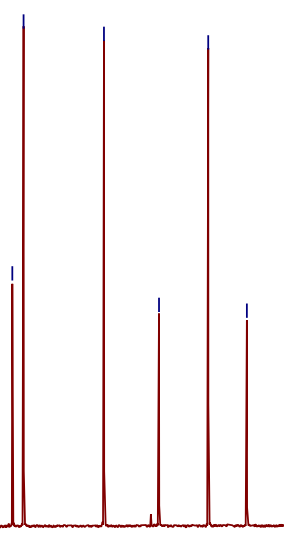




\section{${ }^{1} \mathrm{H}$ NMR spectrum for compound 3af following general procedure $\mathrm{A}\left(500 \mathrm{MHz}, \mathrm{CDCl}_{3}\right)$}

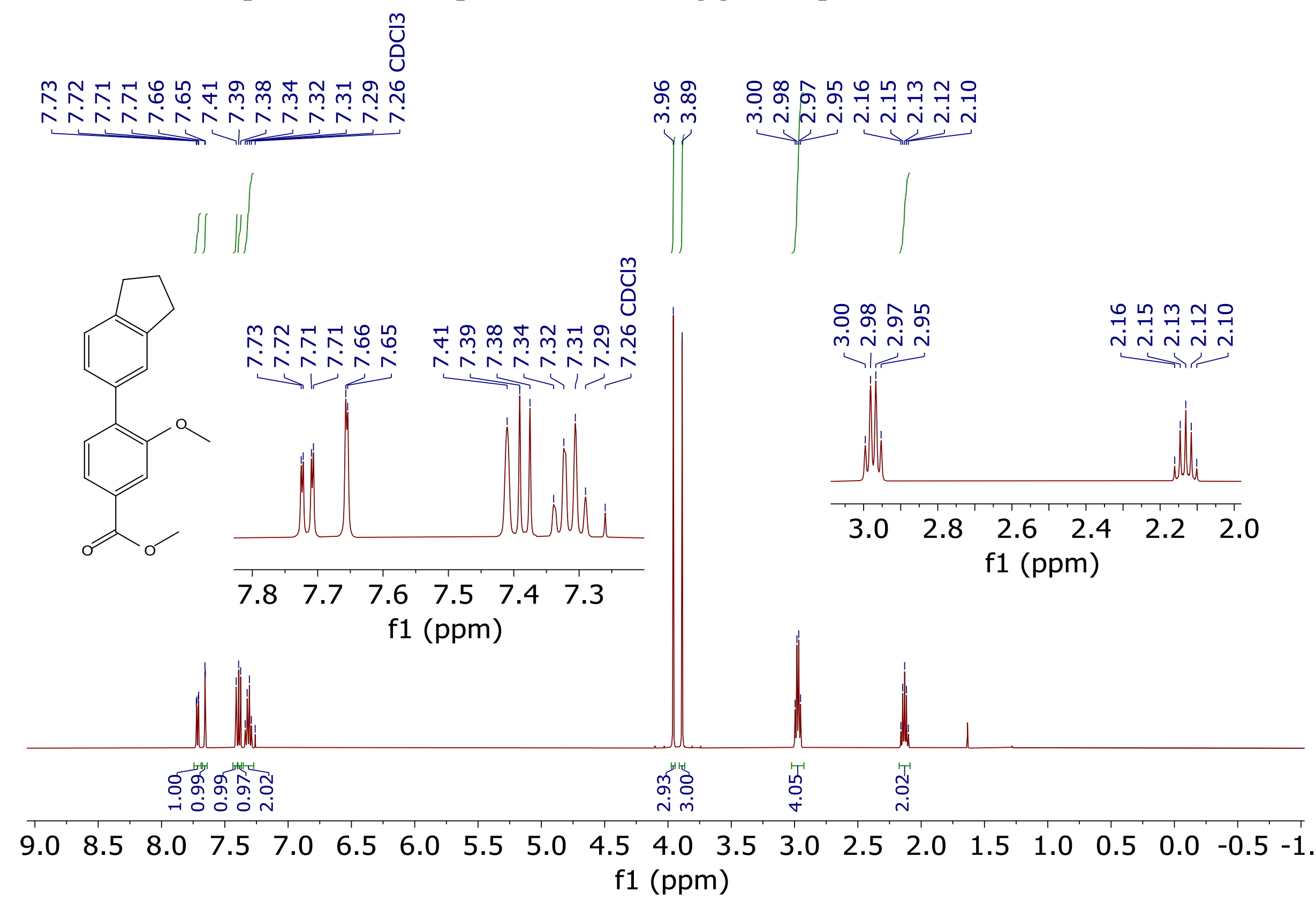


${ }^{13} \mathrm{C}\left\{{ }^{1} \mathrm{H}\right\}$ NMR spectrum for compound 3af following general procedure A (125 $\left.\mathrm{MHz}, \mathrm{CDCl}_{3}\right)$

ન. மீำ

అิ

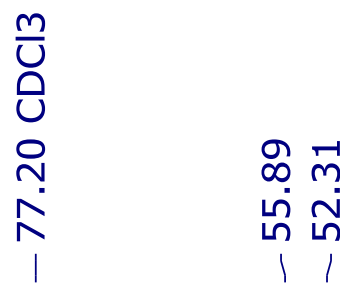

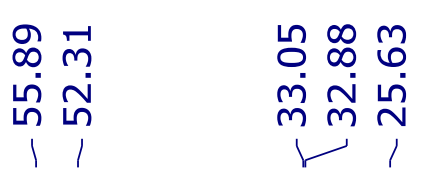
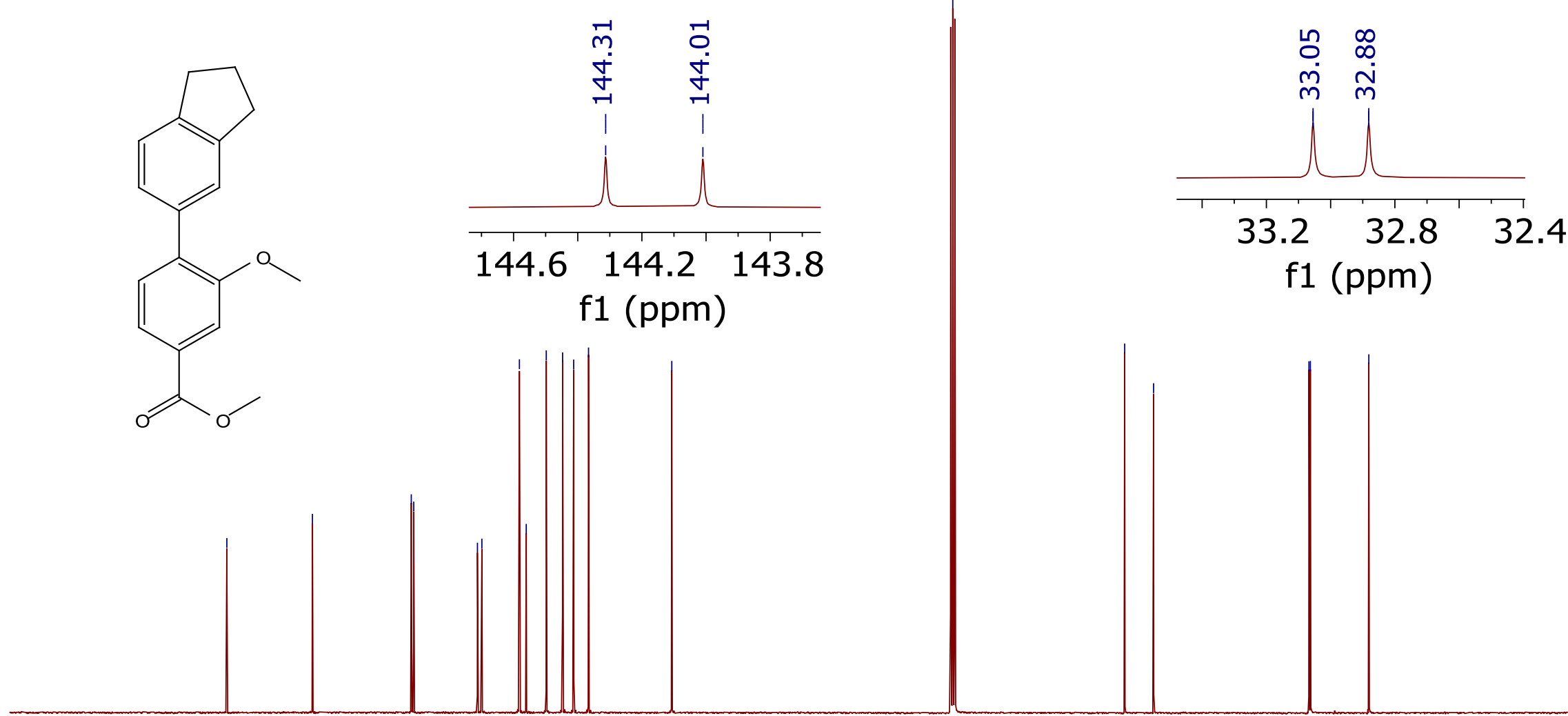

$1(\mathrm{ppm})$

$\begin{array}{llllllllllllllllllll}190 & 180 & 170 & 160 & 150 & 140 & 130 & 120 & 110 & 100 & 90 & 80 & 70 & 60 & 50 & 40 & 30 & 20 & 10 & 0\end{array}$

f1 (ppm) 


\section{${ }^{1} \mathrm{H}-\mathrm{NMR}$ spectrum for compound 3af following general procedure $\mathrm{B}\left(400 \mathrm{MHz}, \mathrm{CDCl}_{3}\right)$}

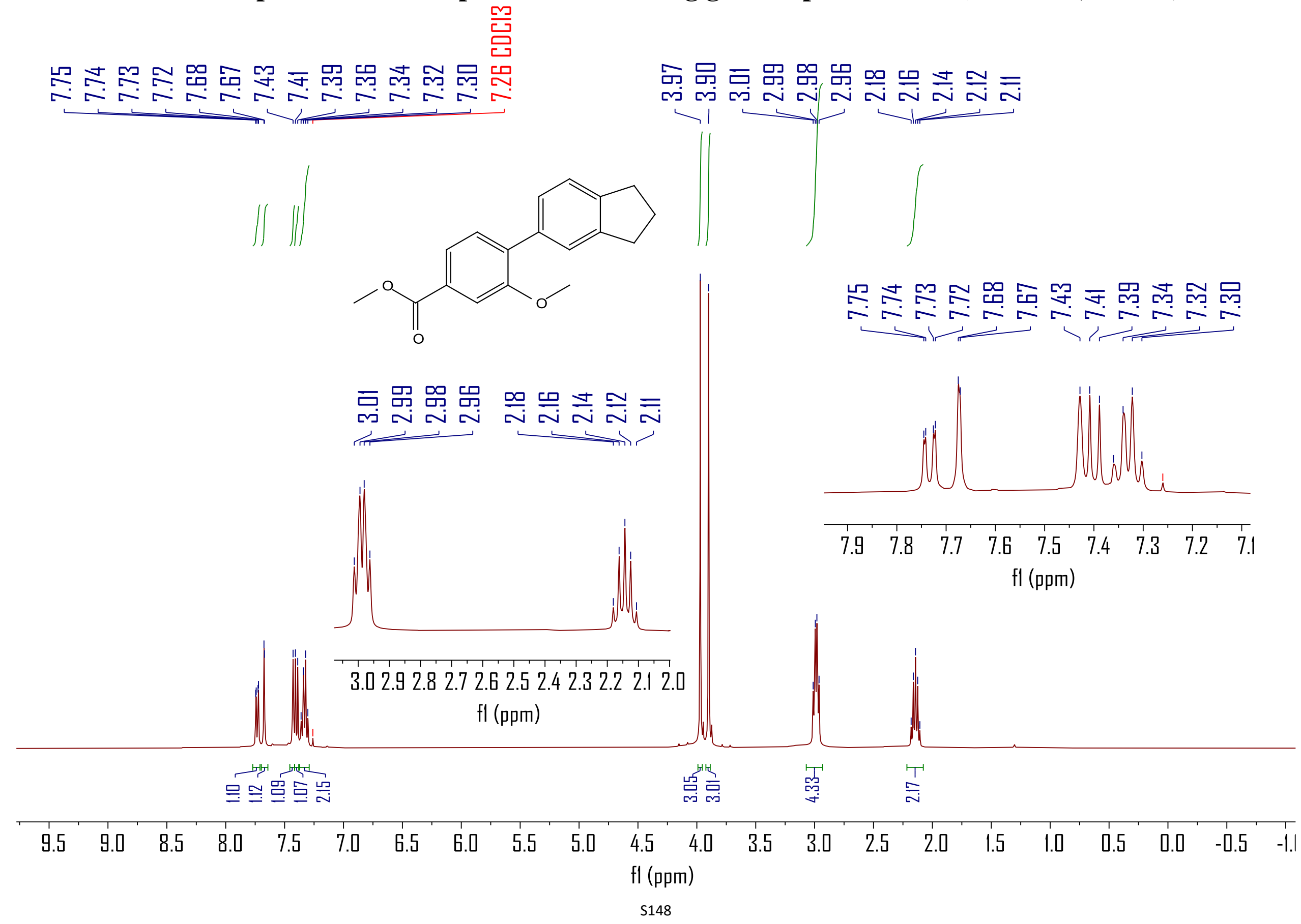


${ }^{13} \mathrm{C}\left\{{ }^{1} \mathrm{H}\right\}$-NMR spectrum for compound 3af following general procedure $\mathrm{B}\left(100 \mathrm{MHz}, \mathrm{CDCl}_{3}\right)$

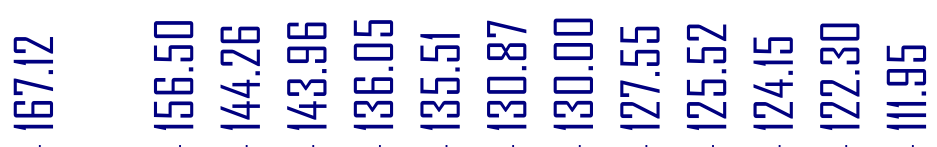
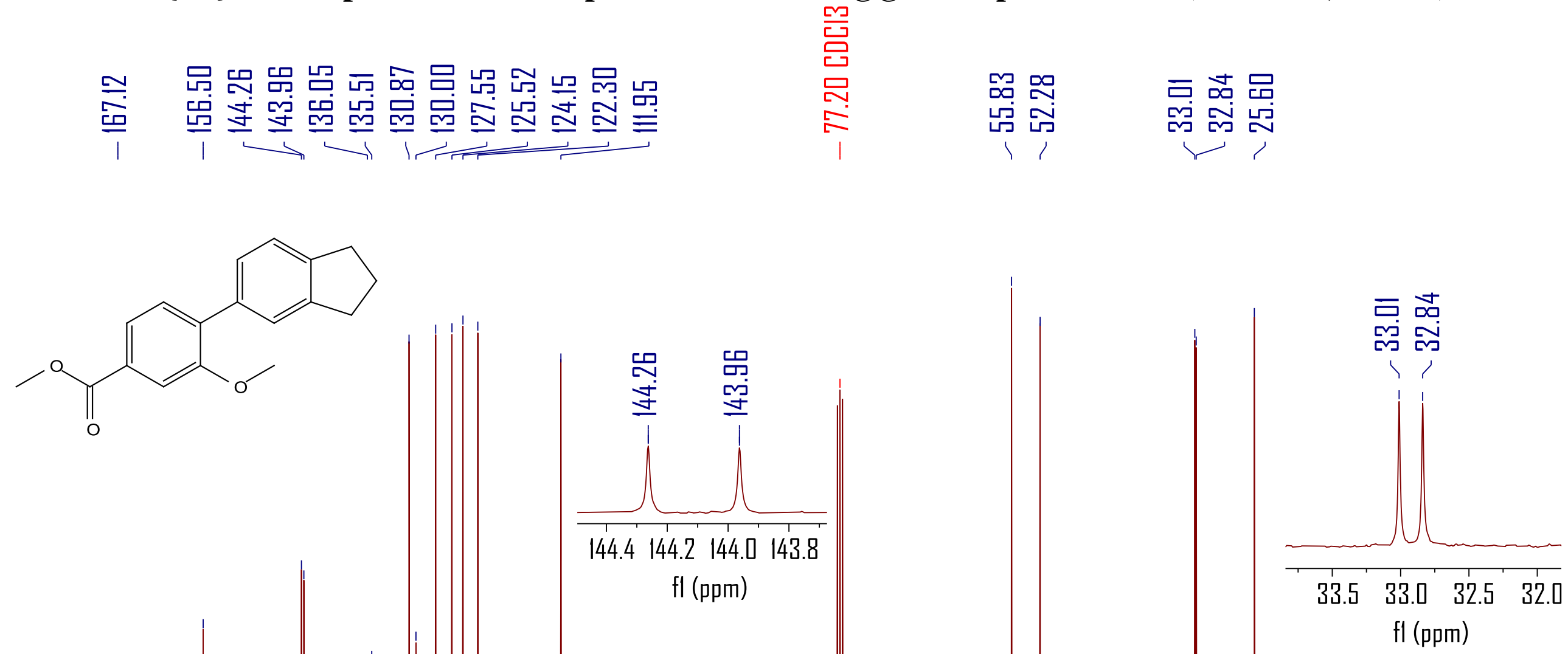

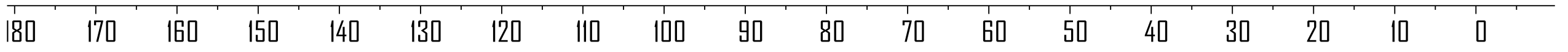




\section{${ }^{1} \mathrm{H}$ NMR spectrum for compound $3 \mathrm{ag}\left(500 \mathrm{MHz}, \mathrm{CDCl}_{3}\right)$}

듬

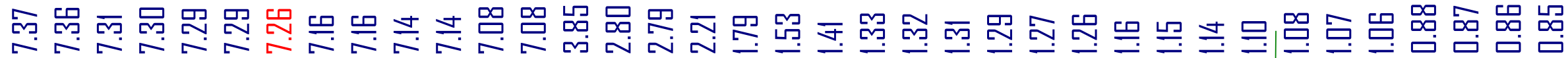
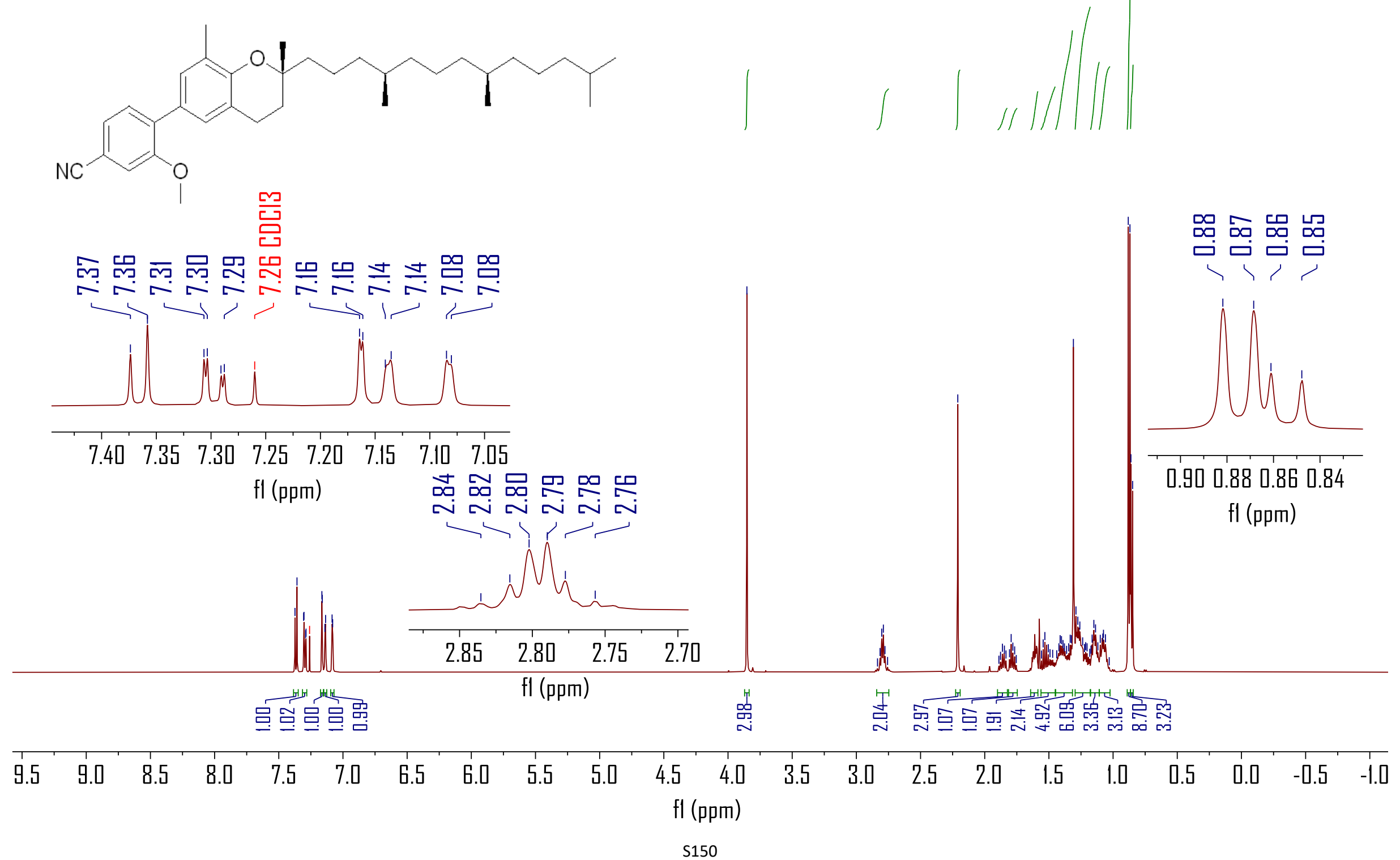


\section{${ }^{13} \mathrm{C}\left\{{ }^{1} \mathrm{H}\right\}$ NMR spectrum for compound 3ag (125 $\left.\mathrm{MHz}, \mathrm{CDCl}_{3}\right)$}

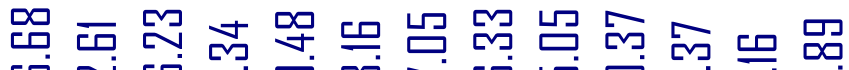

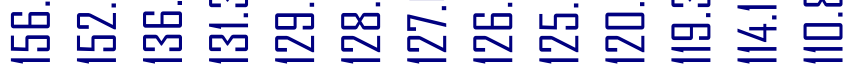

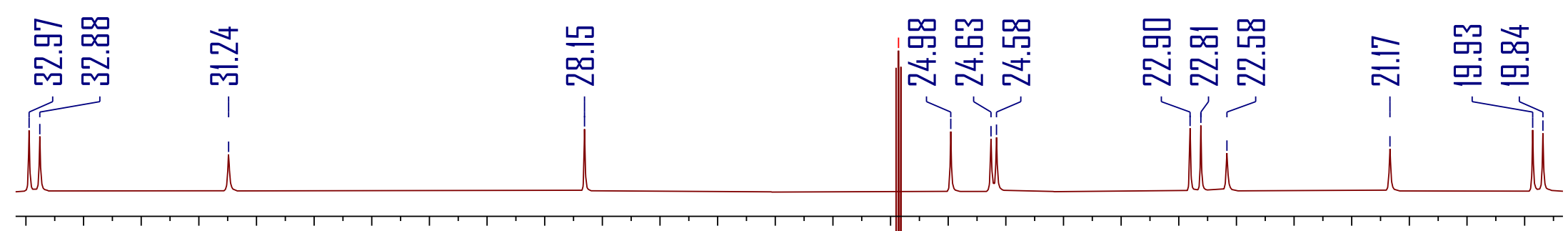

33.032 .532 .031 .531 .030 .530 .029 .529 .028 .528 .027 .527 .026 .526 .025 .5 25.0 24.5 24.0 23.5 23.0 22.5 22.0 21.5 21.0 20.5 20.0

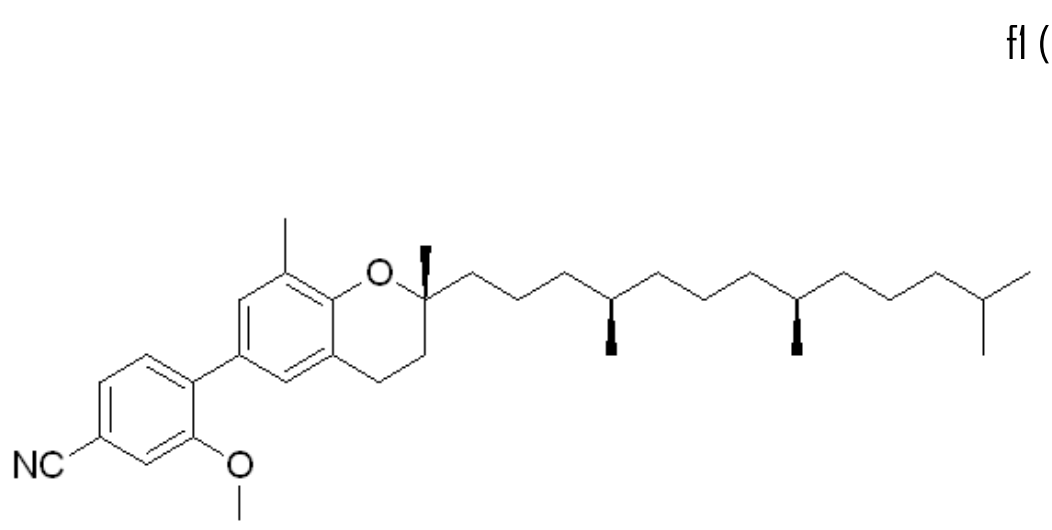

$170 \quad$ IGD $\mathrm{fl}(\mathrm{ppm})$

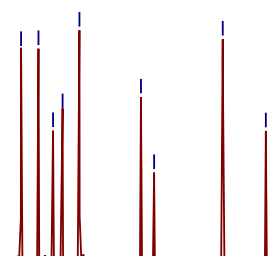

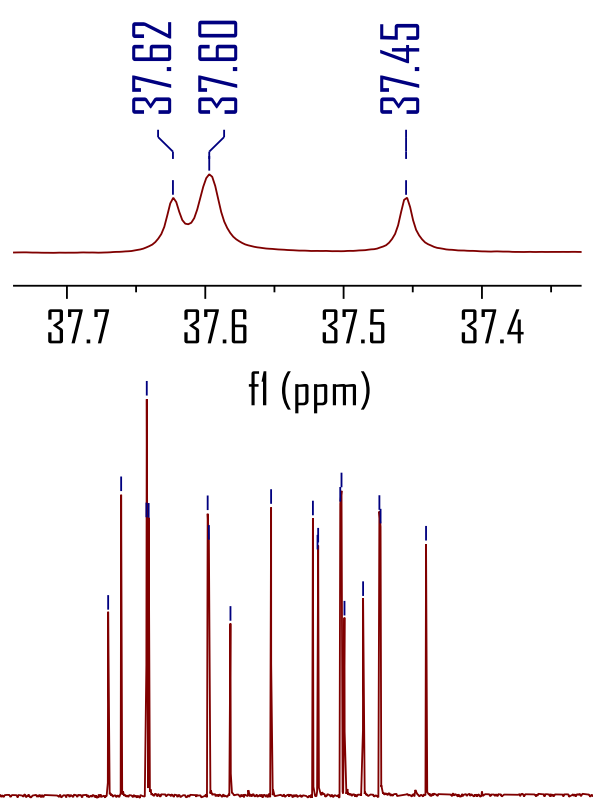


${ }^{1} \mathrm{H}$ NMR spectrum for compound $20\left(500 \mathrm{MHz} \mathrm{CDCl}_{3}\right)$

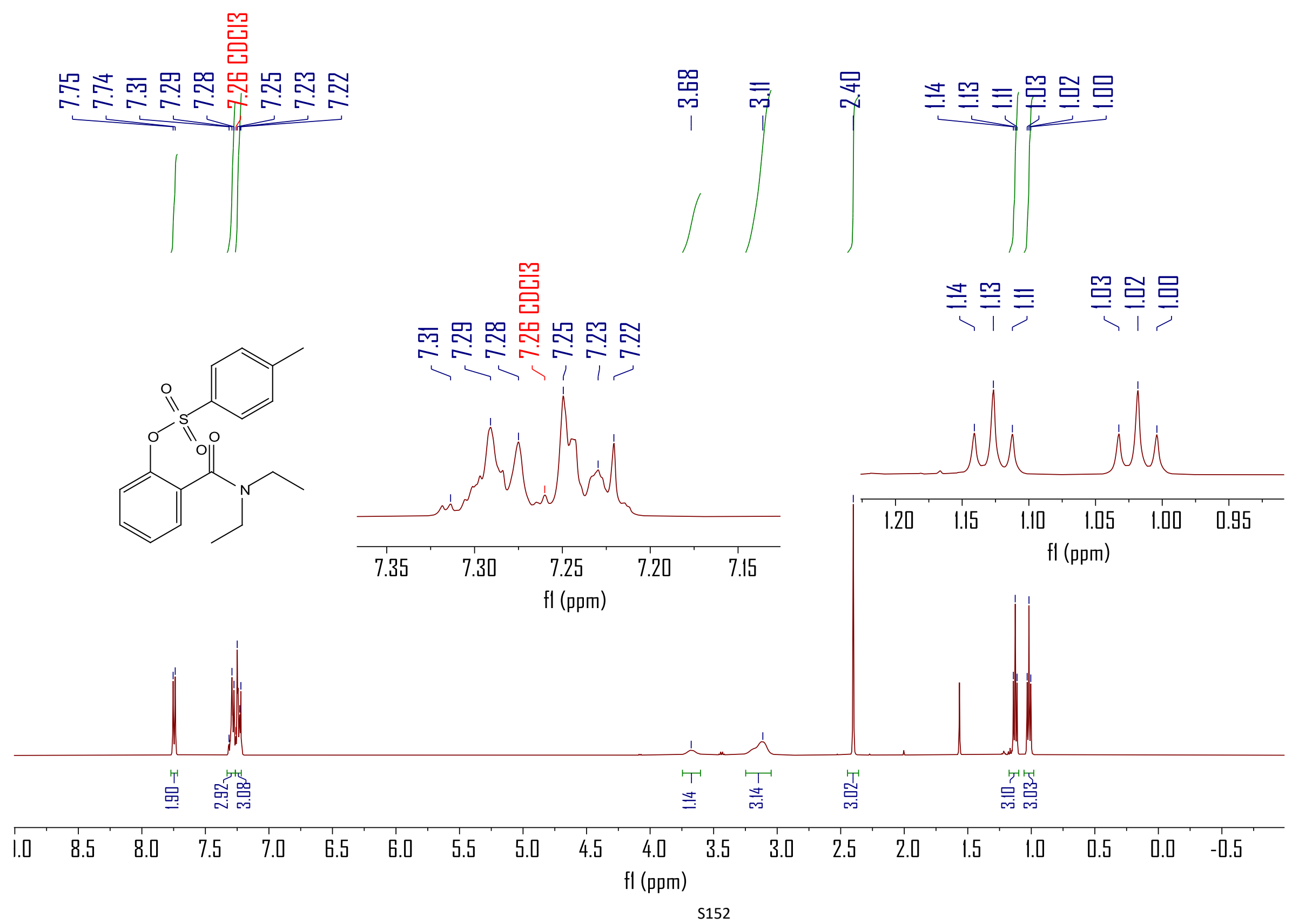




\section{${ }^{13} \mathrm{C}\left\{{ }^{1} \mathrm{H}\right\} \mathrm{NMR}$ spectrum for compound $20\left(125 \mathrm{MHz}, \mathrm{CDCl}_{3}\right)$}

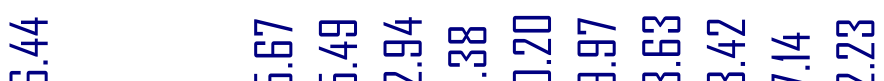

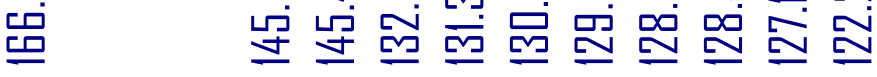
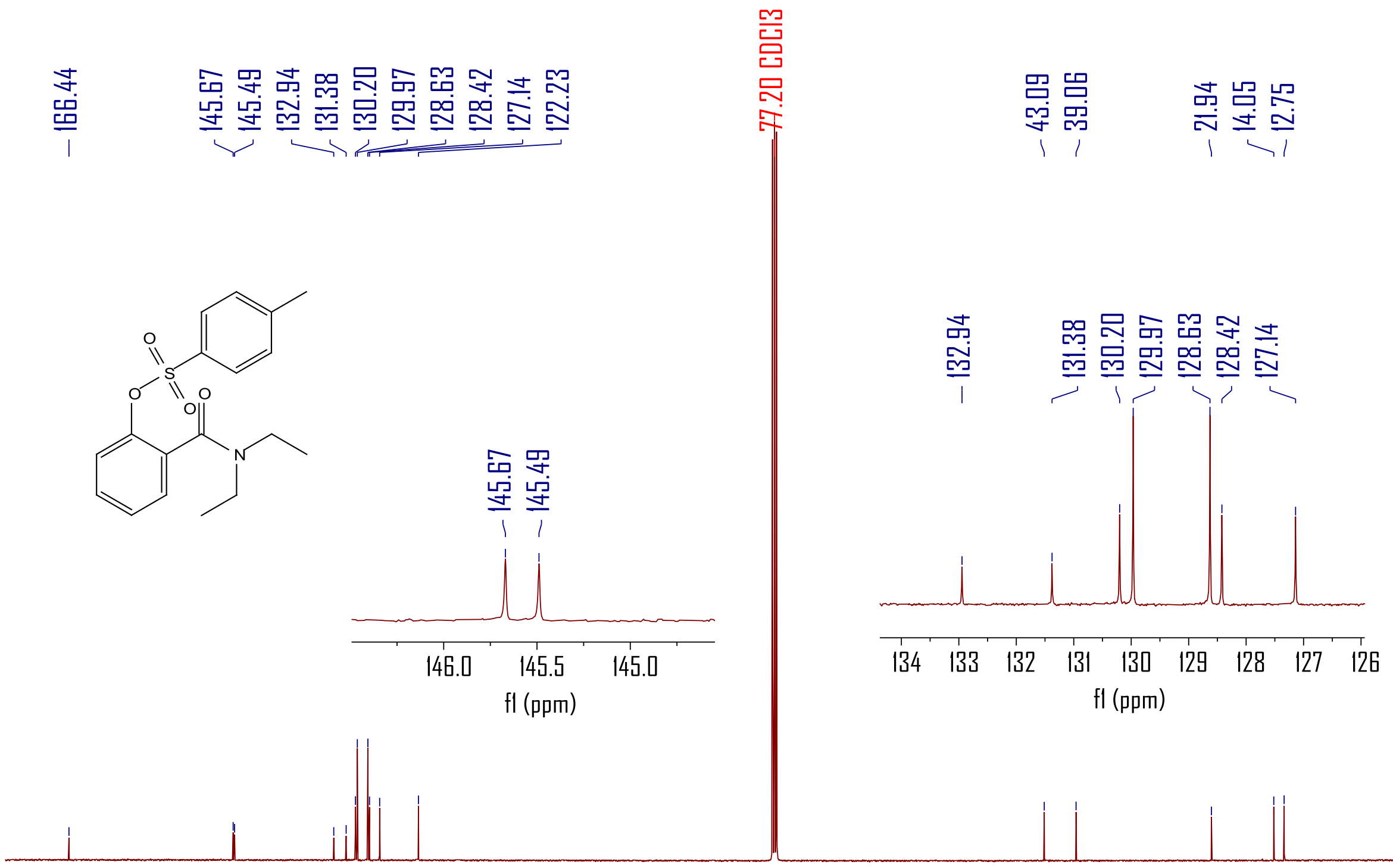

$170 \quad 160 \quad 150 \quad 140 \quad 130$

$30 \quad 120 \quad 110$

100

908

$\mathrm{fl}(\mathrm{ppm})$ 


\section{${ }^{1} \mathrm{H}$ NMR spectrum for compound $1 \mathrm{i}\left(400 \mathrm{MHz}, \mathrm{CDCl}_{3}\right)$}

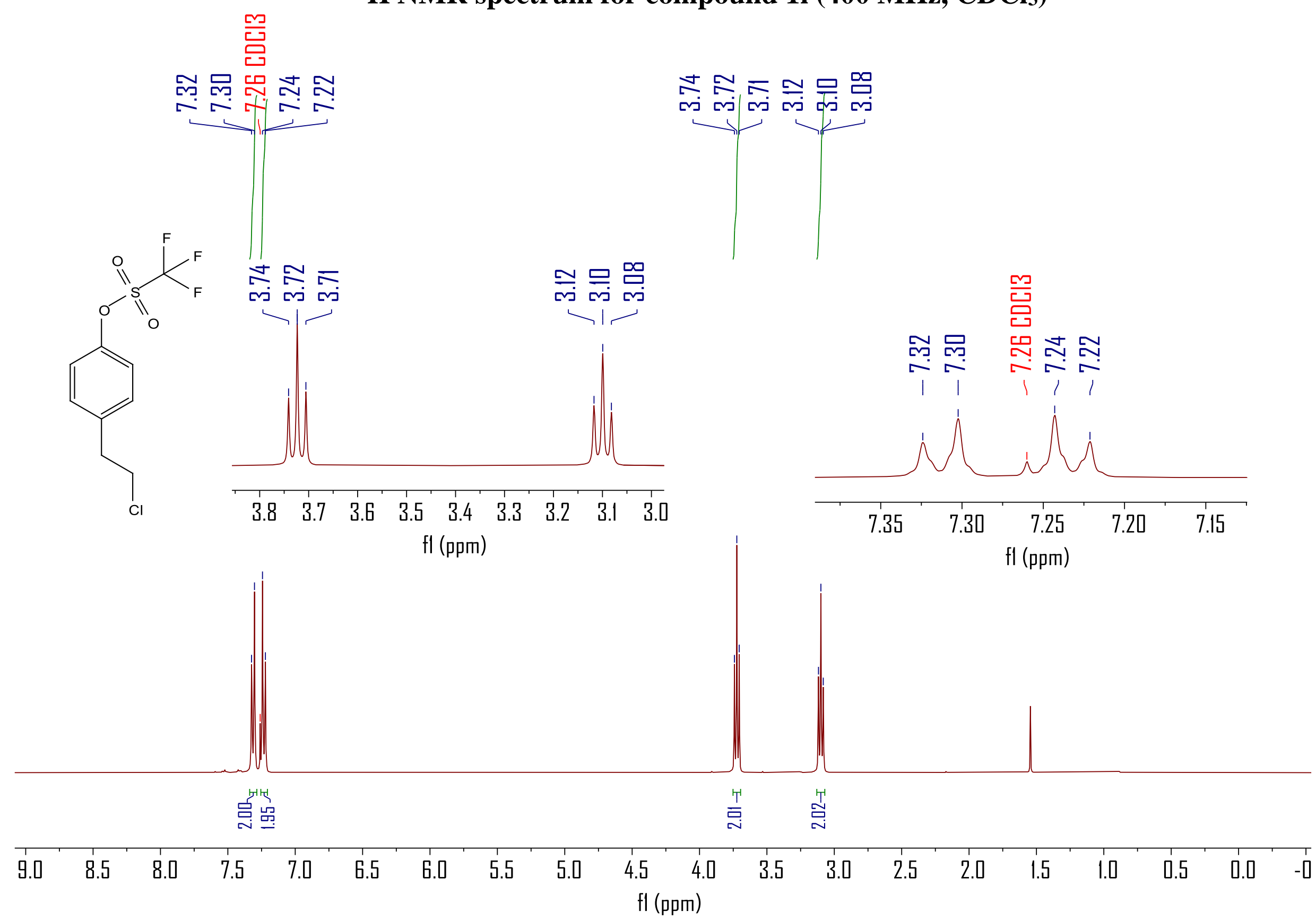


${ }^{19} \mathrm{~F}\left\{{ }^{1} \mathrm{H}\right\}$ NMR spectrum for compound $1 \mathrm{i}\left(376 \mathrm{MHz}, \mathrm{CDCl}_{3}\right)$

㟧
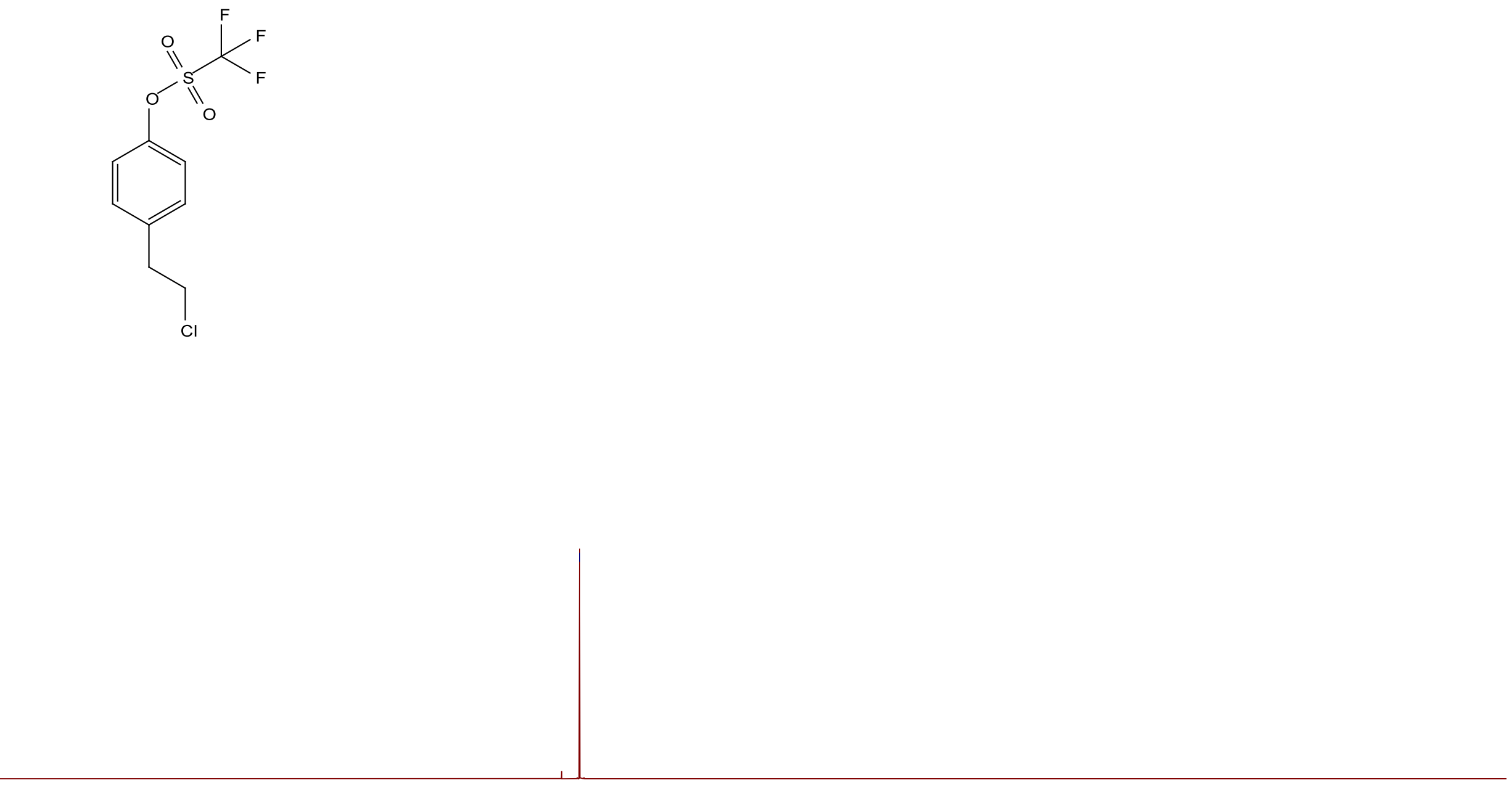

$-90 \quad-100$




\section{${ }^{13} \mathrm{C}\left\{{ }^{1} \mathrm{H}\right\} \mathrm{NMR}$ spectrum for compound $1 \mathrm{i}\left(125 \mathrm{MHz}, \mathrm{CDCl}_{3}\right)$}

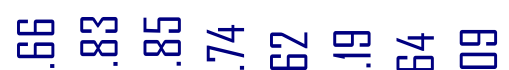

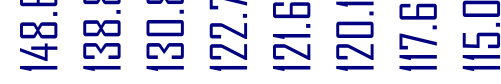
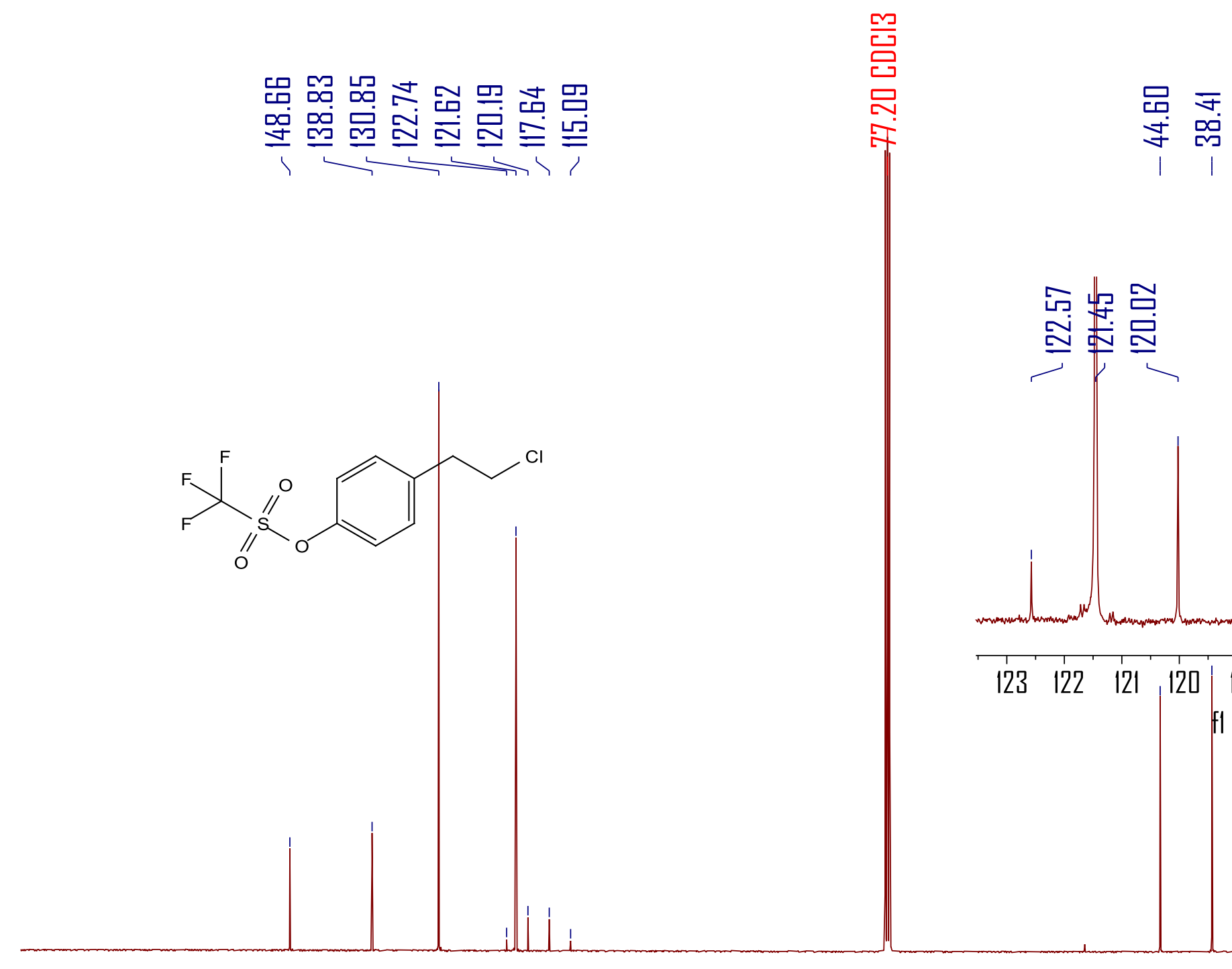

$160 \quad 15$

140130

120

1009

80

$70 \quad 60$

品资

竎 哥

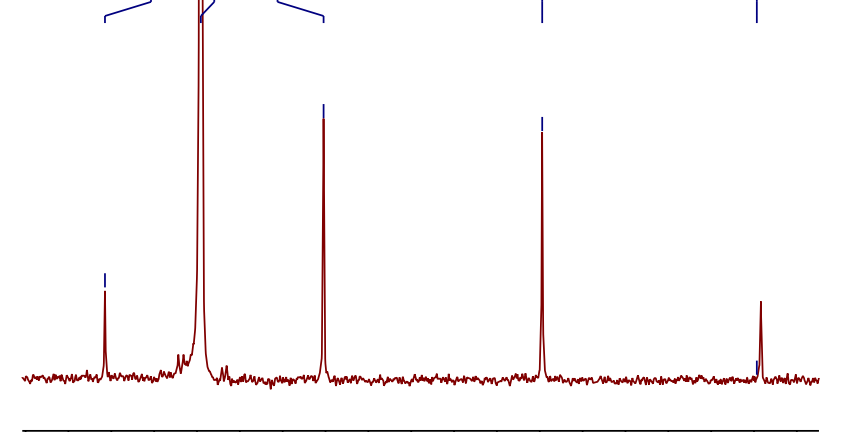

\begin{tabular}{llll|lllll}
123 & 122 & 121 & 120 & 119 & 118 & 117 & 116 & 115
\end{tabular}

fl (ppm)

$\mathrm{fl}$ (ppm) 


\section{${ }^{1} \mathrm{H}$ NMR spectrum for compound 7c (500 $\left.\mathrm{MHz}, \mathrm{CDCl}_{3}, \mathrm{TMS}\right)$}

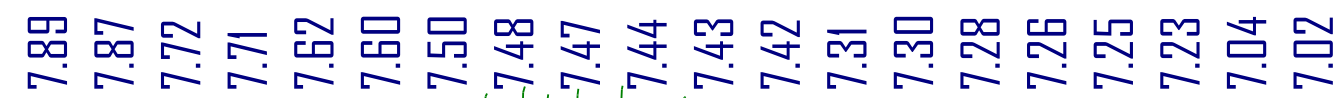

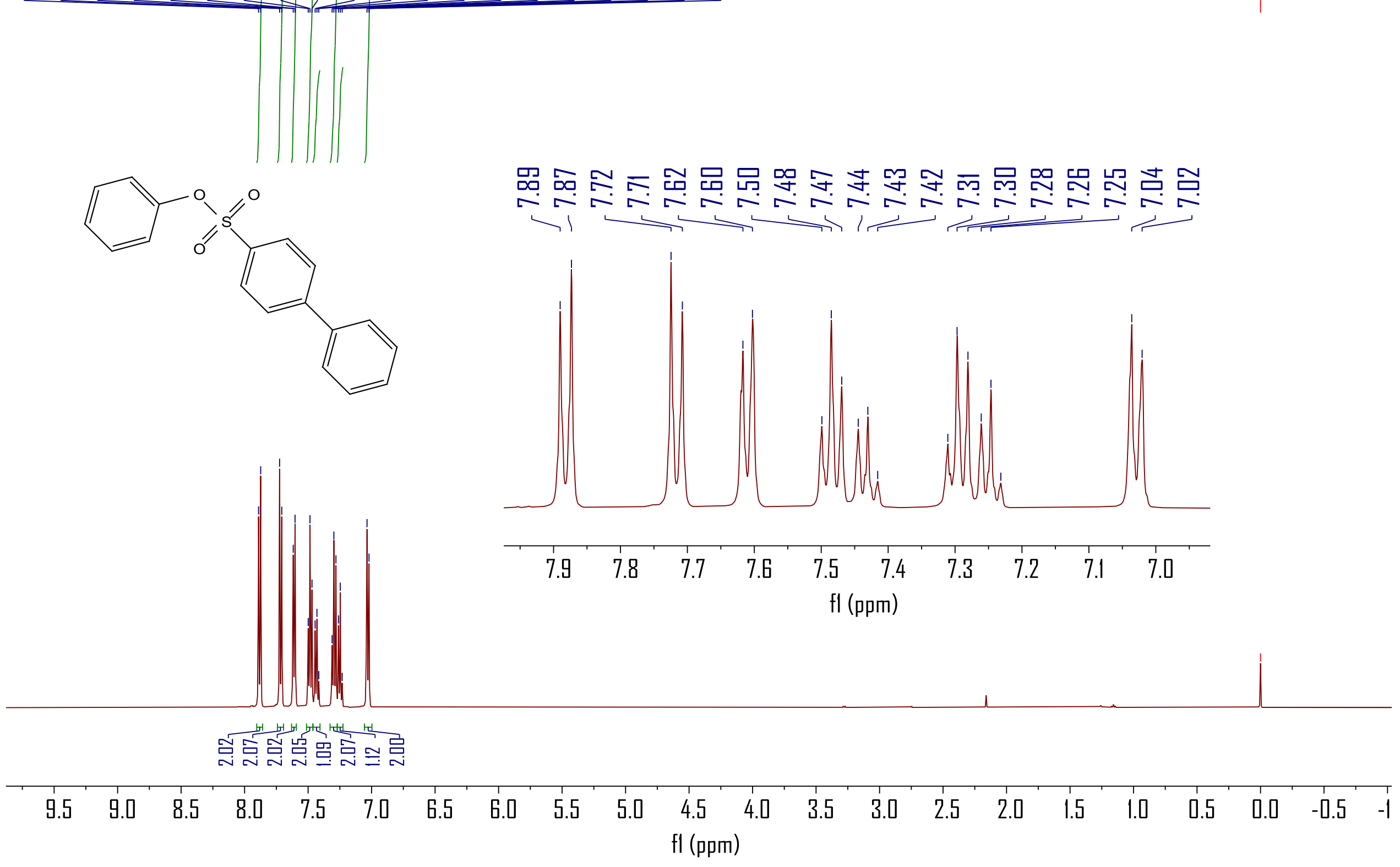




\section{${ }^{13} \mathrm{C}\left\{{ }^{1} \mathrm{H}\right\}$ NMR spectrum for compound 7c (100 $\left.\mathrm{MHz}, \mathrm{CDCl}_{3}\right)$}

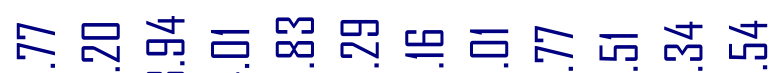

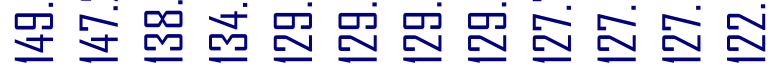

믐

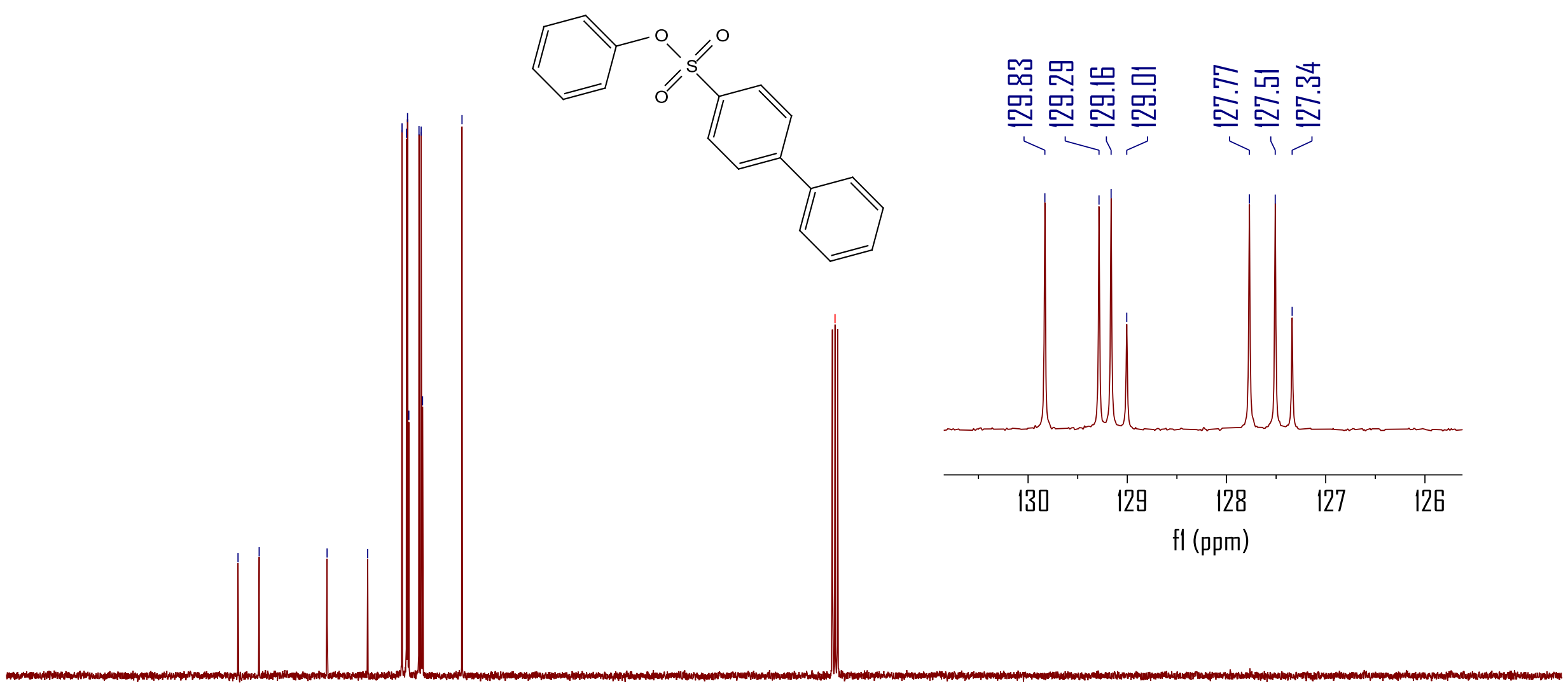

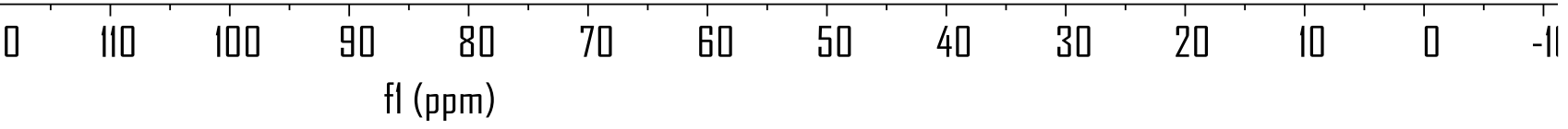




\section{${ }^{1} \mathrm{H}$ NMR spectrum for compound $7 \mathrm{f}\left(500 \mathrm{MHz}, \mathrm{CDCl}_{3}, \mathrm{TMS}\right)$}
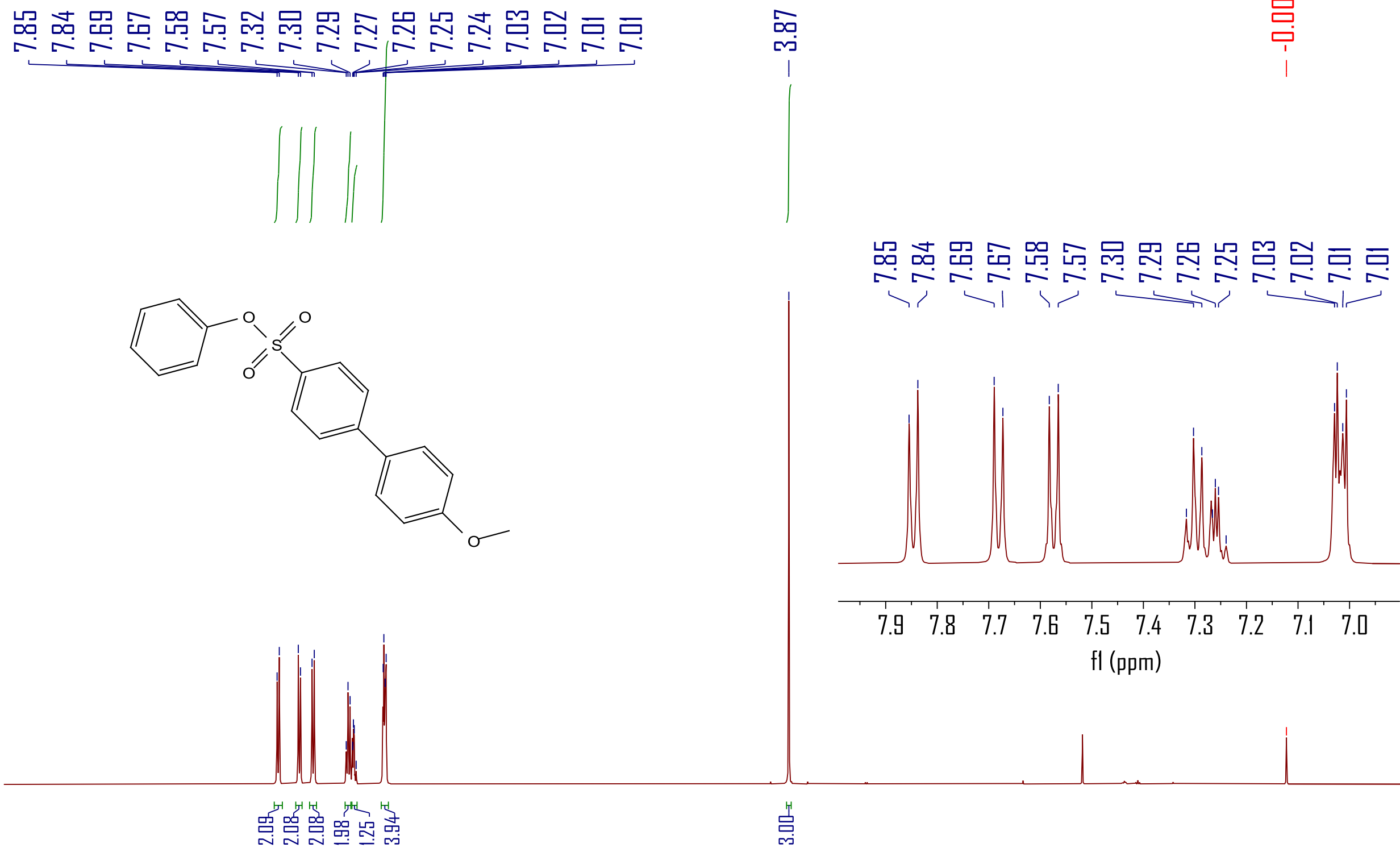

$\begin{array}{llllllllllllllllllllll}9.5 & 9.0 & 8.5 & 8.0 & 7.5 & 7.0 & 6.5 & 6.0 & 5.5 & 5.0 & \begin{array}{c}4.5 \\ \mathrm{fl}(\mathrm{pm})\end{array} & 4.0 & 3.5 & 3.0 & 2.5 & 2.0 & 1.5 & 1.0 & 0.5 & 0.0 & -0.5 & -1.0\end{array}$




\section{${ }^{13} \mathrm{C}\left\{{ }^{1} \mathrm{H}\right\}$ NMR spectrum for compound $7 \mathrm{f}\left(125 \mathrm{MHz}, \mathrm{CDCl}_{3}\right)$}
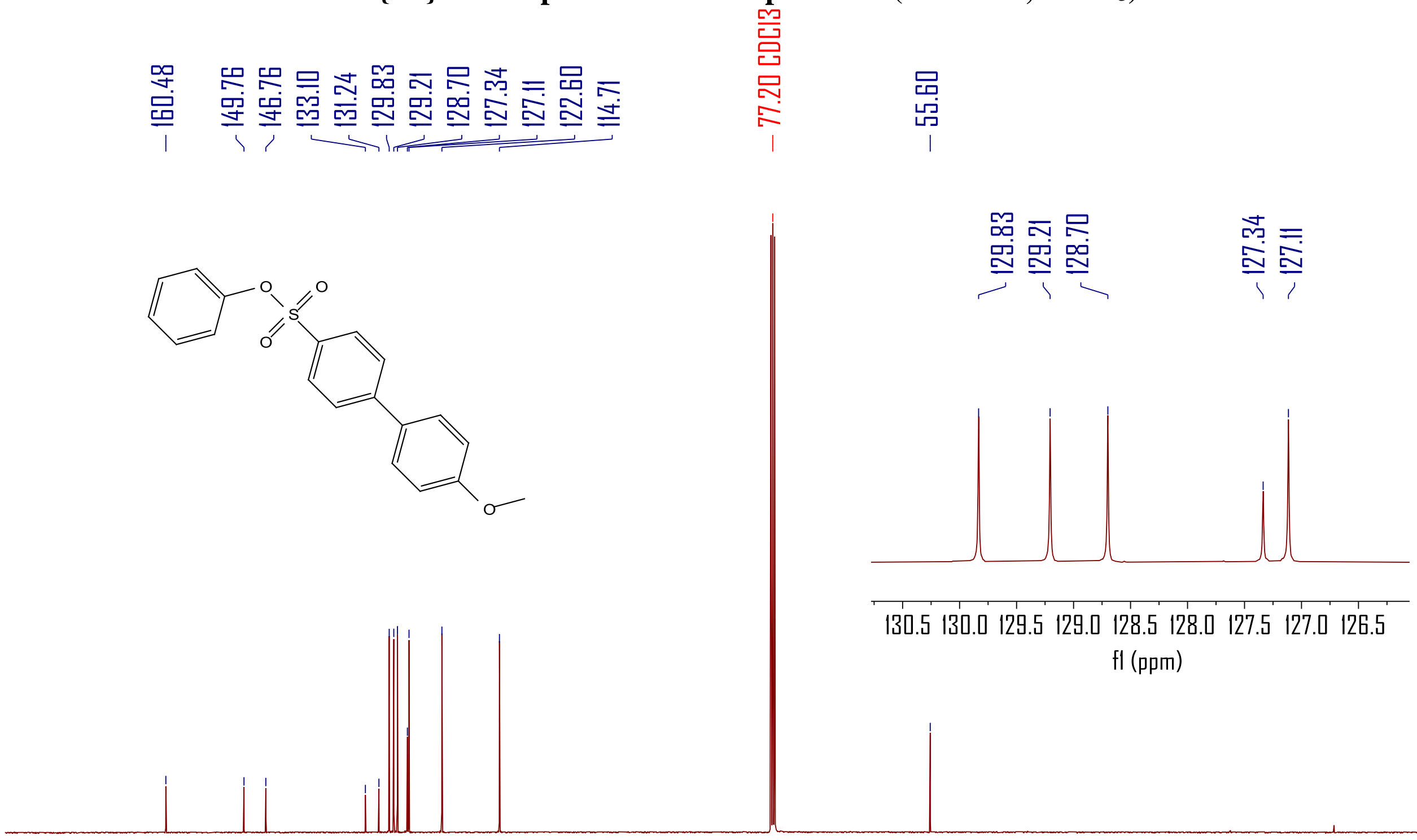

$130.5 \quad 30.0129 .5 \quad 129.0128 .5 \quad 128.0 \quad 127.5 \quad 127.0 \quad 126.5$ $\mathrm{fl}(\mathrm{ppm})$

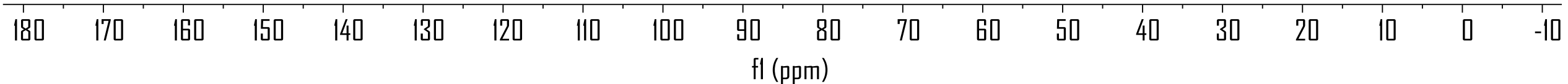




\section{${ }^{1} \mathrm{H}$ NMR spectrum for compound $7 \mathrm{~g}$ (500 $\left.\mathrm{MHz}_{\mathrm{CDCl}}, \mathrm{CMS}\right)$}

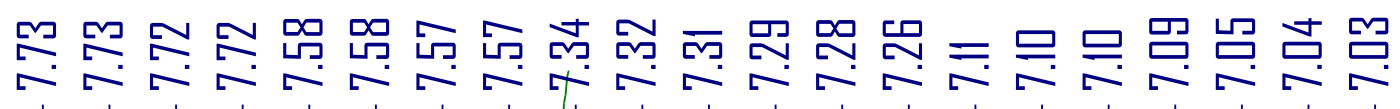

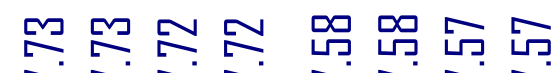

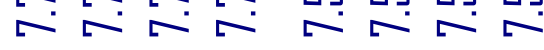

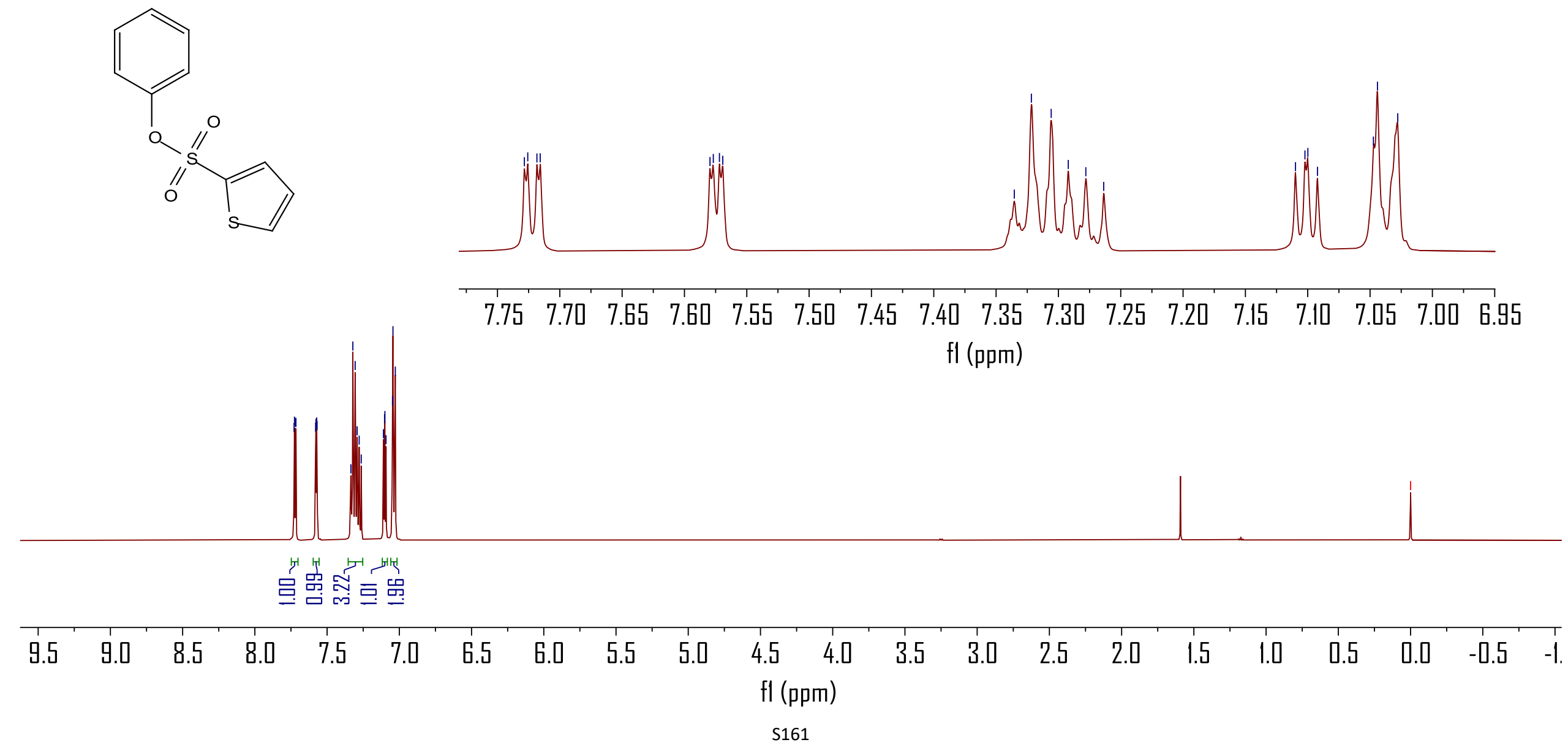

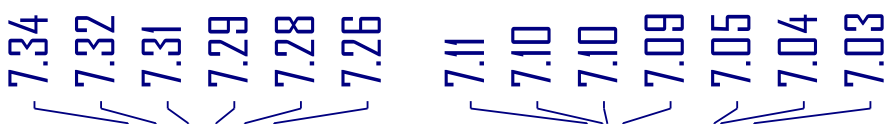




\section{${ }^{13} \mathrm{C}\left\{{ }^{1} \mathrm{H}\right\}$ NMR spectrum for compound $7 \mathrm{~g}\left(125 \mathrm{MHz}, \mathrm{CDCl}_{3}\right)$}

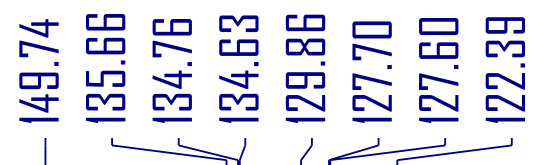
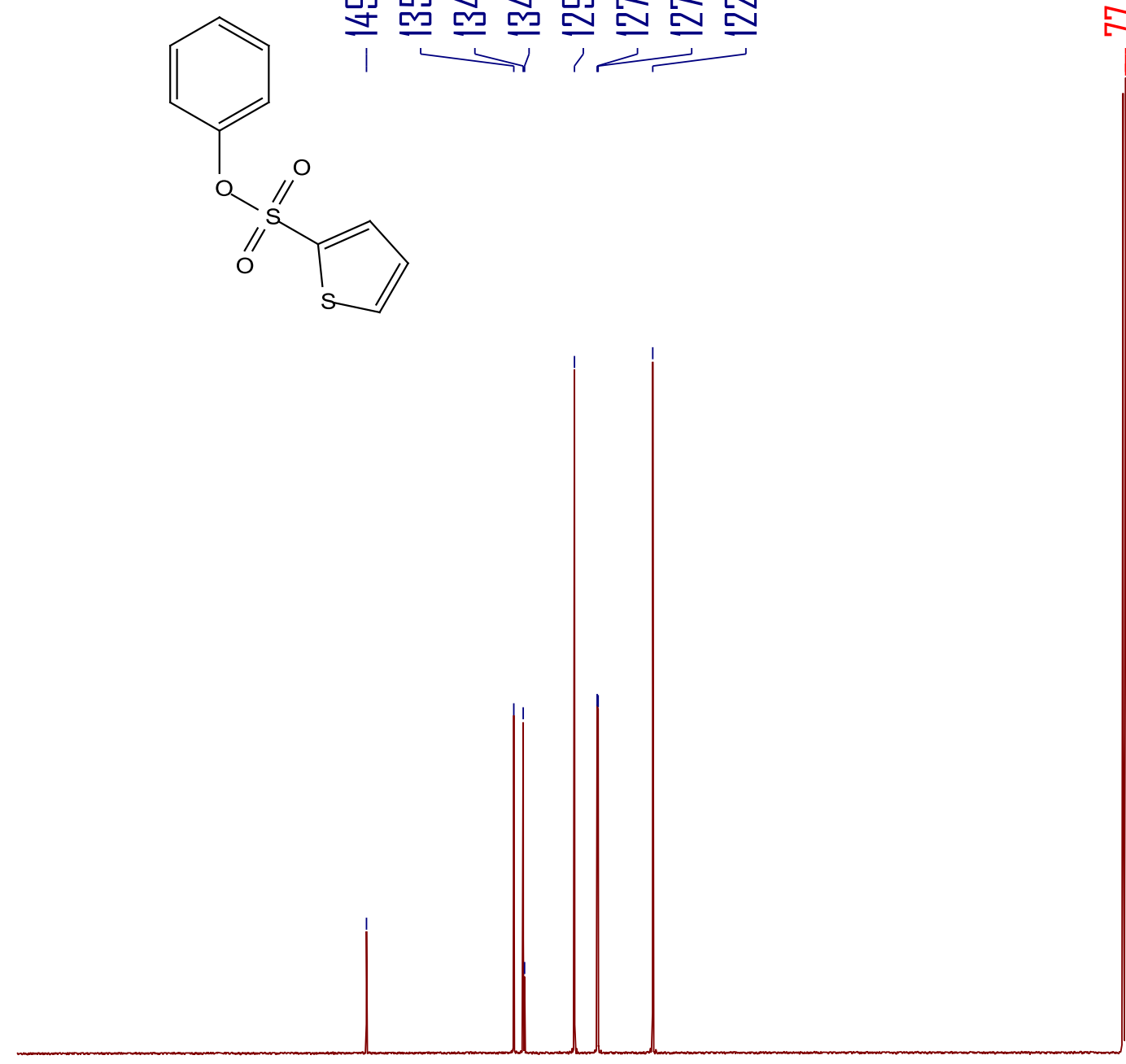
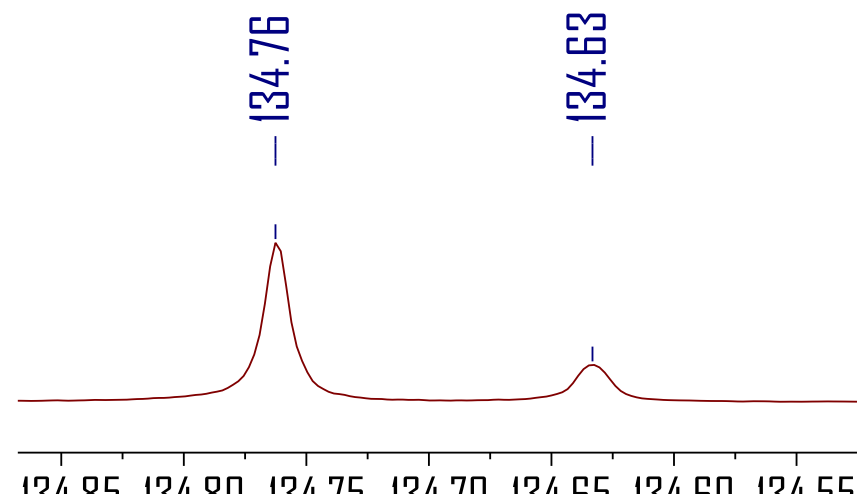

134.85134 .80134 .75134 .70134 .65134 .60134 .55 $\mathrm{fl}(\mathrm{ppm})$
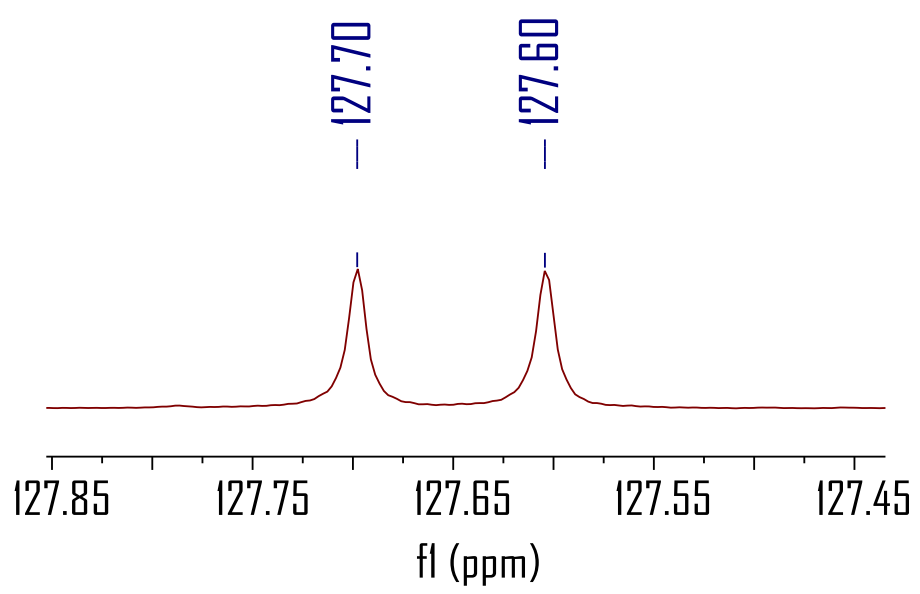

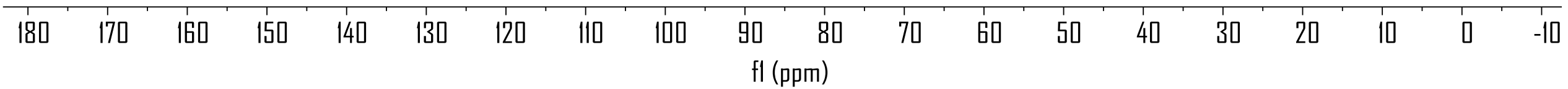

